\title{
Structural determinants of voltage-dependent gating of $K_{v} 10.1$
}

Dissertation for the award of the degree "Doctor rerum naturalium" (Dr.rer.nat.)

of the Georg-August-Universität Göttingen

within the IMPRS Neuroscience program

of the Georg-August University School of Science (GAUSS)

submitted by

Reham Abdelaziz

From Cairo, Egypt

Göttingen, 2020 


\section{Thesis Committee:}

Prof. Dr. Luis Pardo,

Oncophysiology Group, Max-Planck-Institute for Experimental Medicine

Prof. Dr. Martin Göpfert,

Department of Cellular Neurobiology, University of Göttingen

Dr.Andreas Neef, Neurophysics Lab,

Campus Institute for Dynamics of Biological networks, Max-Planck-Institute for

Experimental Medicine

\section{Members of the Examination Board}

Prof. Dr. Ralf Heinrich,

Department of of Cellular Neurobiology, University of Göttingen

Prof. Dr. Dr. h.c. Erwin Neher,

Max-Planck-Institute for Biophysical Chemistry

Prof. Dr. Bert de Groot,

Computational Biomolecular Dynamics, Max-Planck-Institute for Biophysical Chemistry 


\section{Table of contents}

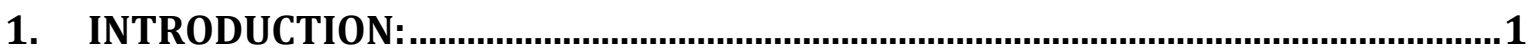

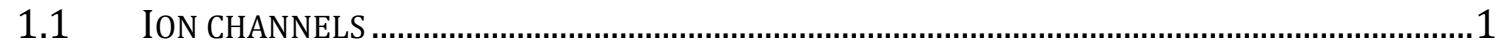

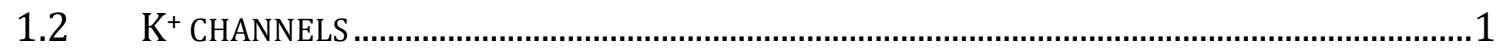

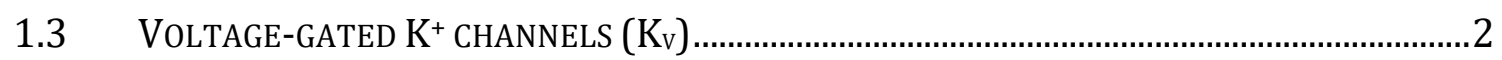

1.4 STRUCTURAL FEATURES OF KCNH (EAG) CHANNELS........................................................

1.4.1 Voltage sensor

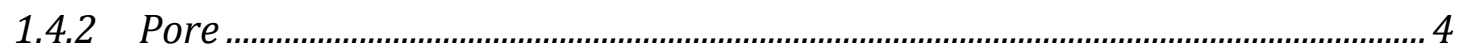

1.4.3 Intracellular domains........................................................................................ 5

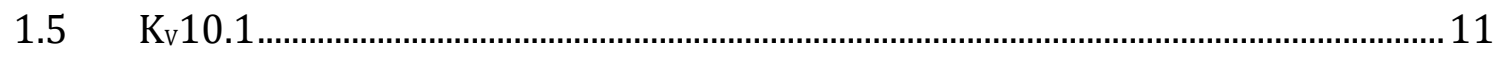

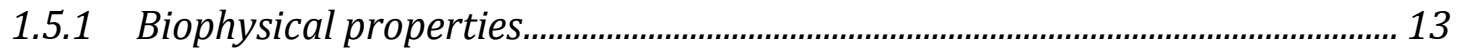

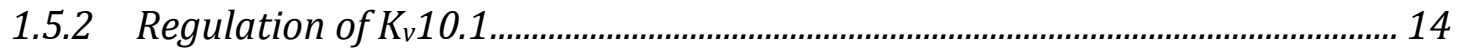

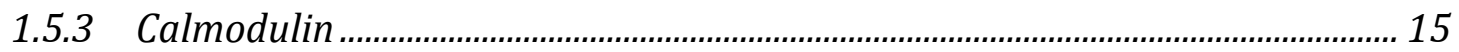

1.6 MODELING OF VOLTAGE-GATED ION CHANNELS ................................................................. 18

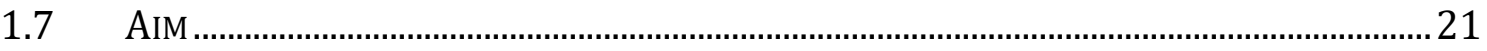

2. METHODS

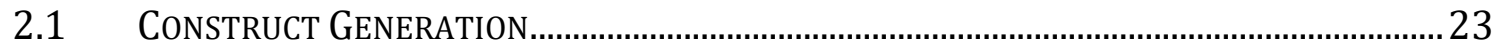

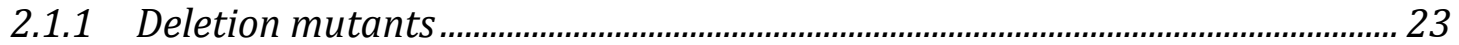

2.1.2 Quick Change mutagenesis (Site-specific mutation).......................................... 24

2.1.3 Cloning into a vector for expression in mammalian cells ................................... 26

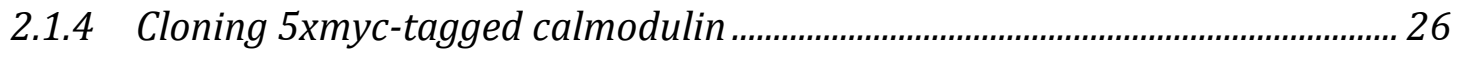

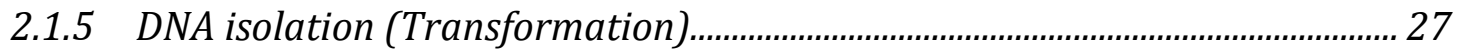

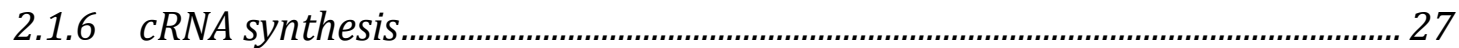

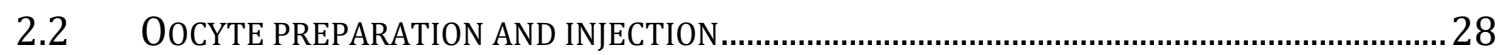

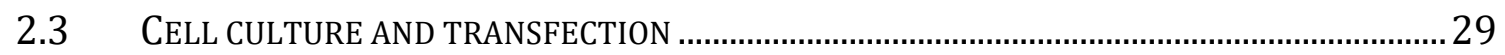

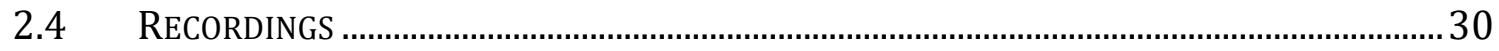

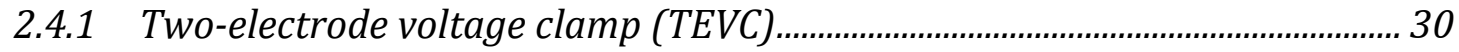

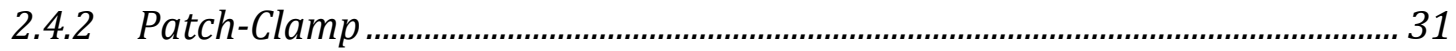




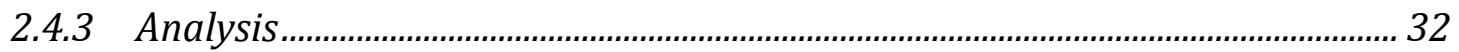

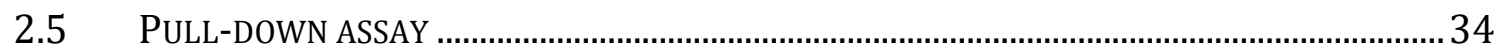

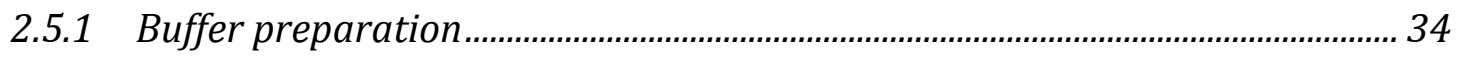

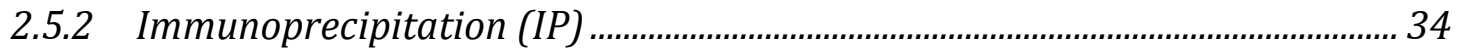

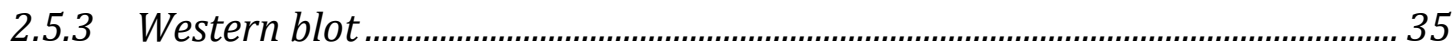

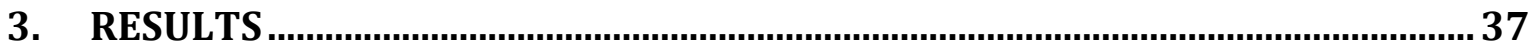

3.1 THE INITIAL N-TERMINUS MUTANT R8C FORMS DISULFIDE BRIDGES WITH ENDOGENOUS

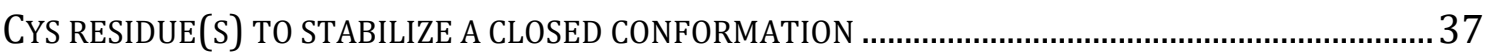

3.2 N-TERMINAL DELETIONS AND E600R UNVEIL A BIPHASIC BEHAVIOR ............................... 41

3.2.1 Deletion of the first ten residues ....................................................................... 41

3.2.2 Larger N-terminal deletions and E600R

3.2.3 Biphasic kinetic behavior in N-terminal deletions and E600R ........................ 49

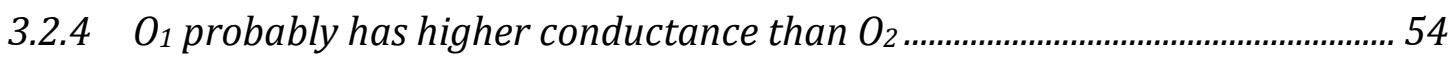

3.2.5 The first component of the biphasic GV represents a common gating step

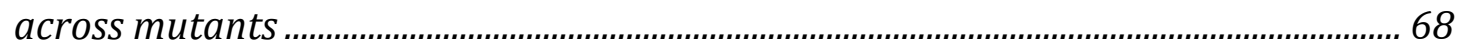

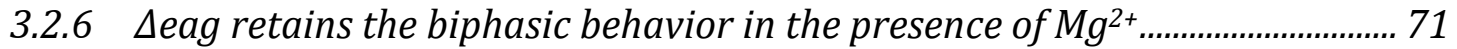

3.2.7 $\Delta 2-10$ loses the biphasic GV upon disrupting a covalent connection between S4 and S5 (L341.split)

3.3 THE FIRST PHASE OF THE BIPHASIC GV IS STABILIZED WITH HYPERPOLARIZED PRE-PULSES AND CA ${ }^{2+-}$ CALMODULIN 75

3.3.1 Hyperpolarized pre-pulse potentiates the first phase of the biphasic IV ...... 75

3.3.2 $\mathrm{Ca}^{2+}-$ Calmodulin stabilizes the first phase of the biphasic IV. 80

3.3.3 The first phase of the biphasic IV is lost when the Calmodulin-binding site BDC2 is disrupted. 85

3.3.4 $\mathrm{Ca}^{2+}$ Calmodulin binding to $\mathrm{K}_{v} 10.1$ at basal $\mathrm{Ca}^{2+}$ level 90

3.4 Second gating Step: A mutation at the end of S4 (D342C) Reproduces the BIPHASIC BEHAVIOR (SHIFTSO 2 TO THE RIGHT).

3.5 $\triangle$ CNBHD REQUIRES STRONGER DEPOLARIZATIONS TO OPEN AND LOSES COLE-MOORE. 94

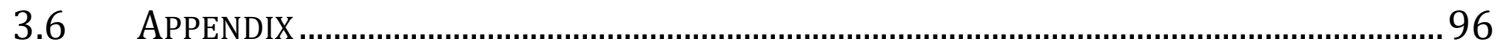

3.6.1 WT tail current kinetics is slower with a weak depolarizing pre-pulse (-20 $m V) \quad 96$ 
4.1 MOBILITY OF THE INITIAL N-TERMinUS IS CRUCIAL FOR THE GATING EVENTS OF $\mathrm{K}_{\mathrm{v}} 10.1$ 98

4.2 DISRUPTING THE INTERACTION BETWEEN PASCAP AND CNBHD REVEALS AN OPEN STATE “ $\mathrm{O}_{1}$ ” 100

4.2.1 Different conductance of $\mathrm{O}_{1}$ and $\mathrm{O}_{2}$. 103

4.2.2 The biphasic behavior may correspond to two-voltage dependent gating events 105

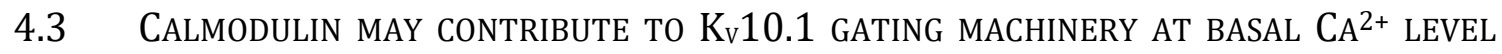
108

4.3.1 The C-lobe of Calmodulin binds Kv10.1 at basal Ca ${ }^{2+}$ levels 108

4.3.2 $\mathrm{Ca}^{2+}$ Calmodulin inhibits $K_{v} 10.1$ through the intracellular ring ......................109

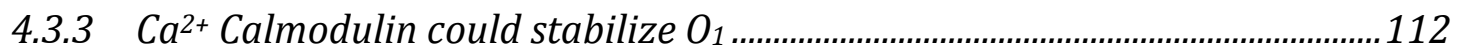

4.4 ROLE OF INTRACELLULAR DOMAINS IN THE GATING OF Kv10.1 ..................................... 114

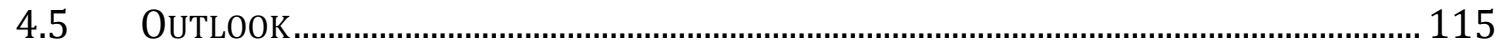

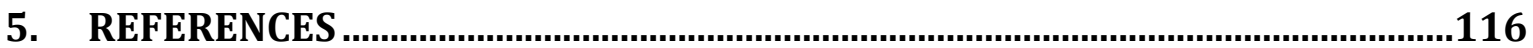

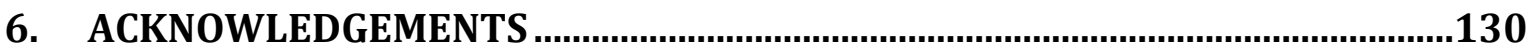

\section{List of Figures}

Figure 1. Cladogram of Voltage-gated $\mathrm{K}^{+}$channels $\left(\mathrm{K}_{\mathrm{v}}\right.$ ) .....................................................

Figure 2. Cryo-EM structure of rat $\mathrm{K}_{\mathrm{v}} 10.1$ (Whicher and MacKinnon, 2016)......................7

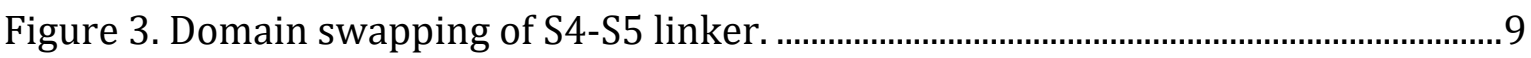

Figure 4. Bottom view of the cryo-EM structure of rat Kv10.1(Whicher and MacKinnon, 2016)

Figure 5. R8C reacts with an endogenous Cys to stabilize a closed conformation........ 40

Figure 6. N-terminal deletion ( $\Delta 2-10)$ unveils a biphasic behavior. 42

Figure 7. Tail current deviates from end-pulse current indicating outward rectification at strong potentials. 43

Figure 8. $\Delta 2-10$ shows similar biphasic behavior in HEK293 cells. 43

Figure 9. $\triangle$ PASCap unmasks a biphasic GV. 46 
Figure 10. $\Delta$ eag reveals a rectifying GV.

Figure 11. E600R replicates the biphasic behavior observed with $\mathrm{N}$-terminal deletions.

Figure 12. Activation kinetics of N-terminal deletions and E600R accelerates with stronger potentials.

Figure 13. N-terminal deletions and E600R show peculiar deactivation kinetics........ 54

Figure 14. Apparent inactivation is evident with longer pulse duration $(\Delta 2-10 \ldots \ldots . . . . .56$

Figure 15. Apparent inactivation is confirmed with varying pre-pulse potentials $(\Delta 2$ $10)$.

Figure 16. Repetition of pre-pulse voltage with fixed duration causes a slight decrease in current amplitude

Figure 17. Apparent inactivation is observed with moderate depolarizing pulses $(\triangle \mathrm{PASCap})$

Figure 18. E600R show apparent inactivation at moderate and strong depolarizations.

Figure 19. Alternating the voltage between weak and strong depolarizations recover E600R and $\Delta$ eag current at strong potentials.

Figure 20. No remarkable difference in $\Delta 2-10$ current amplitude when the voltage was alternated between +80 and $-80 \mathrm{mV}$.

Figure 21. Alternating the voltage between -20 and $50 \mathrm{mV}$ recovers apparent inactivation

Figure 22.WT response to alternating the voltage between weak and strong depolarizations.

Figure 23.Weak depolarizations show higher conductance than strong potentials. Recordings were performed in $60 \mathrm{mM} \mathrm{K}^{+}$solution.

Figure 24.Individual components of a two-sigmoidal fit of the biphasic GV. 70 Figure 25. $\mathrm{Mg}^{2+}$ retains the biphasic behvior and shifts the $\Delta$ eag I-V curve to the right. 72

Figure 26. $\Delta 2-10$. L341split loses the first component of the biphasic GV. 74 Figure 27. N-terminal deletions $(\Delta 2-10 ; \Delta$ PASCap) and E600R activation accelerates with depolarized pre-pulse potentials 76

Figure 28. Hyperpolarizing pre-pulse potentiates $\triangle$ PASCap current at moderate potentials 78 
Figure 29. Hyperpolarizing pre-pulse potentiates E600R current at moderate potentials.

Figure 30. $\mathrm{Ca}^{2+}$-Calmodulin potentiates $\triangle$ PASCap current converting the biphasic IV to a linear one.

Figure 31. $\mathrm{Ca}^{2+}$-Calmodulin potentiates E600R current converting the biphasic IV to a linear one.

Figure 32. $\mathrm{Ca}^{2+}$-Calmodulin initially inhibits $\Delta 2-10$ then potentiates the first phase of the biphasic IV. 84

Figure 33. $\Delta 2-10$ loses the biphasic GV when BD-C2 is mutated (F714SF717S) 86

Figure 34. $\Delta 2-10$ F714SF717S monophasic IV confirms the loss of $\Delta 2-10$ biphasic GV.

Figure 35. $\triangle$ PASCap loses the biphasic GV when BD-C2 is mutated (F714SF717S) ..... 88

Figure 36. F714SF717S shows a similar GV to the WT 89

Figure 37. Hyperpolarized potentials fail to potentiate $\triangle$ PASCap current when BDC2 is mutated. 90

Figure 38. Pull-down of Kv10.1 with 5xmyc-Calmodulin at $100 \mathrm{nM} \mathrm{Ca}^{2+}$. 91

Figure 39. D342C induces biphasic behavior in Kv10.1 93

Figure 40. D342C Cole-Moore is shifted to the left. 94

Figure 41. $\triangle \mathrm{CNBHD}$ shifts GV to the right and loses Cole-Moore. 95

Figure 42. Depolarized pre-pulse slows down the deactivation of WT. 97

Figure 43. The N-terminus of Kv10.1 lies in the vicinity of endogenous Cys residues.

Figure 44. PASCap and CNBHD interaction surface that could stabilize a closed conformation. 103

Figure 45. Proposed model for Kv10.1 gating 107

Figure 46. The C-lobe of calmodulin may bind to BD-C2 at basal $\mathrm{Ca}^{2+}$ level. 109

Figure 47. Calmodulin interacts with three subunits. 111

Figure 48. Proposed model for $\mathrm{Ca}^{2+}$-Calmodulin inhibition. 113 


\section{List of Tables}

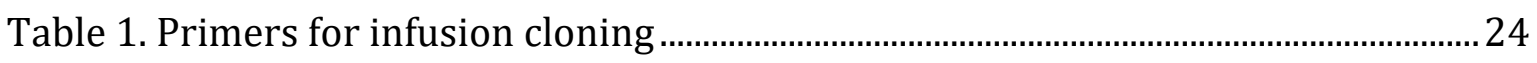

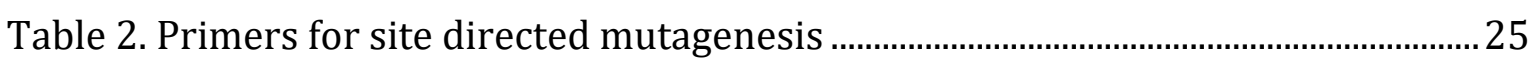

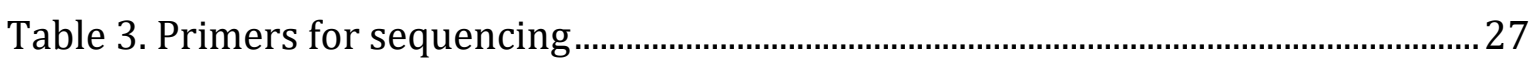

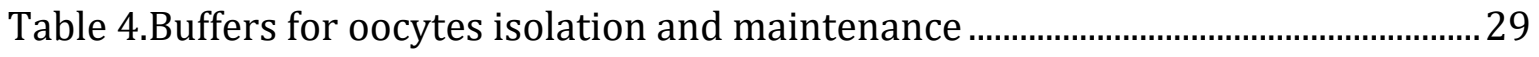

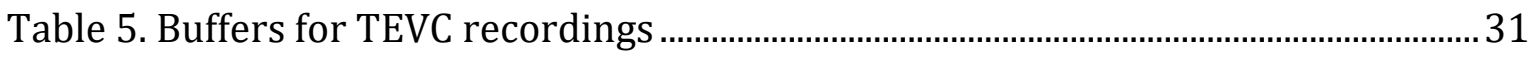

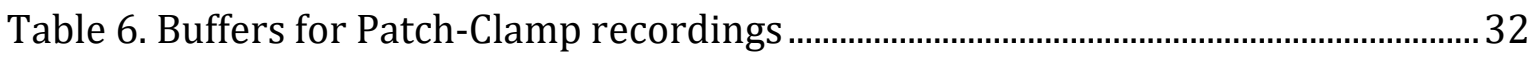

Table 7. Buffers for immunoprecipitation................................................................................ 34

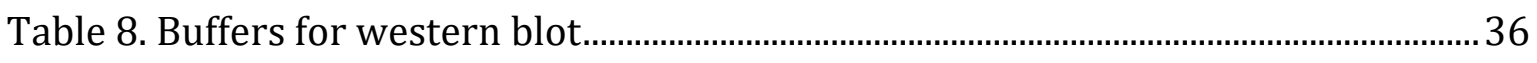

Table 9. Parameters of a global fit that linked the first component of the biphasic

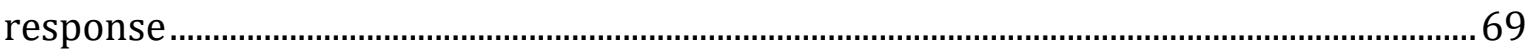




\section{List of Abbreviations}

\begin{tabular}{|c|c|}
\hline A & Active state \\
\hline $\mathrm{BD}-\mathrm{C} 1$ & C-terminal calmodulin binding site 1 \\
\hline $\mathrm{BD}-\mathrm{C} 2$ & C-terminal calmodulin binding site 2 \\
\hline BD-N & $\mathrm{N}$-terminal calmodulin binding site \\
\hline C. & Closed conformation \\
\hline CaM & Calmodulin \\
\hline CaMK & Calmodulin-activated protein kinase \\
\hline CNBD & cyclic nucleotide binding domain \\
\hline CNBHD & cyclic nucleotide binding homology domain \\
\hline CNG & Cyclic nucleotide-gated channels \\
\hline CNS & Central Nervous System \\
\hline cRNA & Complementary RNA \\
\hline cryo-EM & Cryogenic electron microscopy \\
\hline $\mathrm{Da}$ & Dalton \\
\hline DNA & Deoxyribonucleic acid \\
\hline DMEM & Dulbecco's Modified Eagle Medium \\
\hline DMSO & Dimethylsulfoxide \\
\hline DTT & Dithiotreitol \\
\hline $\mathrm{dH}_{2} \mathrm{O}$ & distilled water \\
\hline dNTP & Deoxy-nucleotides triphosphate \\
\hline EAG & Ether à go-go family (eag channels: $K_{v} 10.1$ ) (eag -domain) \\
\hline EDTA & Ethylenediaminetetraacetic acid \\
\hline EGTA & (ethylene glycol-bis( $\beta$-aminoethyl ether)- $\mathrm{N}, \mathrm{N}, \mathrm{N}^{\prime}, \mathrm{N}^{\prime}$-tetraacetic acid) \\
\hline EF hand & Helix-loop-Helix domain that binds $\mathrm{Ca}^{2+}$ \\
\hline ELK & EAG like $\mathrm{K}$ channel \\
\hline ERG & EAG-related gene \\
\hline FBS & Fetal Bovine Serum \\
\hline FRET & Fluorescence resonance energy transfer \\
\hline G & Conductance \\
\hline$\Delta \mathrm{G}$ & Activation energy \\
\hline GFP & Green fluorescent protein \\
\hline GV & Conductance-Voltage relationship \\
\hline $\mathrm{HCN}$ & Hyperpolarization-activated cyclic nucleotide-gated \\
\hline HEPES & 4-(2-hydroxyethyl)-1-piperazineethanesulfonic acid \\
\hline HRP & Horseradish peroxidase \\
\hline $\mathrm{I}-\mathrm{V}$ & Current-voltage relationship \\
\hline IL & Intrinsic ligand \\
\hline IP & Immunoprecipitation \\
\hline $\mathrm{Kbp}$ & Kilo base pairs \\
\hline KCNA & Potassium channel, subfamily A \\
\hline $\mathrm{KCNH}$ & Potassium channel, subfamily $\mathrm{H}$ \\
\hline KO & Knock out \\
\hline $\mathrm{K}_{2 \mathrm{p}}$ & Two-pore domain $\mathrm{K}^{2+}$ channels \\
\hline $\mathrm{K}_{V}$ & Voltage-gated $\mathrm{K}^{+}$channels \\
\hline LB & Luria-Bertani broth \\
\hline LDS & Lithium dodecyl sulphate \\
\hline
\end{tabular}




$\begin{array}{ll}\text { O } & \text { Open conformation } \\ \text { P }_{0} & \text { Probability of channel being open } \\ \text { P }_{c} & \text { Probability of channel being closed } \\ \text { PAS } & \text { Per-Arnt-Sim domain } \\ \text { PCF } & \text { Patch-Clamp fluorometry } \\ \text { PCR } & \text { Polymerase chain reaction } \\ \text { R } & \text { Resting state } \\ \text { RNA } & \text { Ribonucleic acid } \\ \text { TbHO2 } & \text { Tert-butyl hydroperoxide } \\ \text { TEVC } & \text { Two-electrode voltage clamp } \\ \text { TM } & \text { Transmembrane segments } \\ \text { tm-FRET } & \text { Transition metal ion Fluorescence resonance energy transfer } \\ \text { VDP } & \text { Voltage-dependent potentiation } \\ \text { Veq } & \text { Equilibrium potential } \\ \text { Vm } & \text { Membrane potential } \\ \text { Vh } & \text { Voltage that elicits. half maximal response } \\ \text { VSD } & \text { Voltage-sensing domain } \\ \text { WT } & \text { Wild-type } \\ \gamma & \text { Single channel conductance }\end{array}$

\section{Amino acids:}

Three-letter code

Arg

Asp

Cys

Glu

Gln

Leu

Lys

Pro

Phe

Ser

Tyr

Val
One-letter code

R

D

C

E

Q

L

K

$\mathrm{P}$

$\mathrm{F}$

$\mathrm{S}$

Y

V
Residue

Arginine

Aspartate

Cysteine

Glutamate

Glutamine

Leucine

Lysine

Proline

Phenylalanine

Serine

Tyrosine

Valine 


\section{Abstract}

Voltage-gated ion channels sense voltage through the movement of the voltage sensor in response to change in membrane potential, hence influencing the channel pore. In this project, we seek a deeper understanding of electromechanical coupling in a voltage gated $\mathrm{K}^{+}$channel: Kv10.1 (EAG1). In most voltage-gated $\mathrm{K}$ channels (Kv), the loop between transmembrane segments 4 and 5 (S4-S5 linker) acts as a mechanical lever that couples the movement of the sensor to gate opening. However, recent evidence sets apart the KCNH family (Kv10-12) from this model and proposes a distinct mechanism of gating, where the S4 segment itself, rather than the linker, transduces the movement of the sensor. Nevertheless, the complexity of the gating behaviour of this family of channels is not fully explained by this difference and prompts to the existence of additional mechanisms. In an attempt to identify such alternative mechanisms, we turned our attention to the intracellular ring that characterizes this family and is formed by parts of the large $\mathrm{N}$ - and $\mathrm{C}$ - terminal intracellular domains. This intracellular ring shows functional and physical interactions with the core of the channel and has been proposed to participate in gating. We generated partial deletions of intracellular domains, generated synthetic cRNA and characterized the properties of the current using two-electrode voltage clamp in Xenopus laevis oocytes. Deletions in both $\mathrm{N}$ - and C-terminal domains induce profound alterations in channel kinetics, further reinforcing the notion of their participation in gating. Our data indicates that both the voltage sensor and the ring play a role in electromechanical coupling in $\mathrm{KCNH}$ channels. 


\section{Introduction}

\subsection{Ion channels}

Ion channels provide a hydrophilic pathway for ions to cross the hydrophobic environment of the phospholipid bilayer. The permeation pathway is controlled by a gate, usually coupled to a sensor; except for leak channels, the gate opens or closes in response to stimuli (Hille, 2007). In excitable cells, the flow of ions across the membrane can generate an electrical signal (action potential) if a threshold is exceeded. The change in membrane permeability to $\mathrm{Na}^{+}$and $\mathrm{K}^{+}$ions shape the action potential (Hodgkin and Huxley, 1952a, b). It was initially hypothesized that protein carriers transfer ions from one side of the membrane to the other. In contrast to carriers, ion channels allow a rapid flow of ions at a rate of $10^{6}$ ions per second. Ion channels can be classified according to their ion permeability $\left(\mathrm{Na}^{+}, \mathrm{Ca}^{+}, \mathrm{K}^{+}\right)$and the stimulus they respond to (voltage, ligands, mechanical) (Hille, 2007). In this study, we focus on a voltage-gated $\mathrm{K}^{+}$channel: Kv10.1 (EAG1).

\section{$1.2 \mathrm{~K}^{+}$channels}

$\mathrm{K}^{+}$channels set the membrane resting potential and drive hyperpolarization during an action potential. At rest, the membrane is highly permeable to $\mathrm{K}^{+}$ions (through $\mathrm{K}^{+}$selective leak channels), which drives the membrane potential close to the equilibrium potential of $\mathrm{K}^{+}$(normally $\sim-80 \mathrm{mV}$ ). In response to sufficiently intense depolarizing signals, voltage-gated $\mathrm{K}^{+}$channels $\left(\mathrm{K}_{\mathrm{v}}\right)$ open with a delay with respect to $\mathrm{Na}+$ channels. $\mathrm{K}_{\mathrm{v}}$ channels end the depolarizing signal; therefore, the delay in their response determines the action potential duration (Hille, 2007). The heterogeneity of $\mathrm{K}^{+}$channels' kinetics allows them to confer neurons with distinct excitability and firing frequency. The variety in $\mathrm{K}^{+}$channels is set by the different genes encoding them $(80$ 
genes), alternative splicing, and heteromeric assembly (Bauer and Schwarz, 2018; Villa and Combi, 2016).

$\mathrm{K}^{+}$channels can form multimers. They can be classified according to the number of transmembrane segments (TM) in one subunit. $\mathrm{K}^{+}$channels consist of ion conducting (alpha) and accessory (beta) subunits that modify the gating properties. Based on the structure of the alpha subunit, K channels can be classified into a) 2 TM (inward rectifiers), b) 4TM (two-pore domain) c) 6TM (voltage-gated $\mathrm{K}^{+}$channels). The inward rectifiers (Kir) are formed by only the pore domain: two transmembrane segments (S5S6) and a P-loop in between. The two-pore domain channels (K2p) have a repeat of the pore domain in one subunit. Voltage-gated $\mathrm{K}^{+}$channels $\left(\mathrm{K}_{\mathrm{V}}\right)$ consist of $6 \mathrm{TM}$ (S1-S6); a voltage sensor of 4 TM (S1-S4) is associated with one pore domain (S5-S6) to endorse it with voltage sensitivity. $\mathrm{K}_{\mathrm{v}}$ channels assemble as tetramers; four subunits assemble to form the pore in the center with an intracellular gate, while the four sensors are at the periphery (Gonzalez et al., 2012; Hille, 2007).

\subsection{Voltage-gated $\mathrm{K}^{+}$channels $\left(\mathrm{K}_{\mathrm{v}}\right)$}

Voltage-gated $\mathrm{K}^{+}$channels $\left(\mathrm{K}_{\mathrm{v}}\right)$ are a superfamily that comprises a diversity of families. They retain a basic architecture of trans-membrane domains (S1-S6) and vary in intracellular domains and accessory subunits (Hille, 2007). $K_{v}$ families with a cyclicnucleotide binding domain (CNBD) at the C-terminus share structural features that distinguish their gating properties from the rest of $\mathrm{K}_{v}$ channels $\left(\mathrm{K}_{v} 1-\mathrm{K}_{v}\right.$ 9: Shakerrelated channels). This subgroup includes a) Cyclic nucleotide-gated channels (CNG), b) Hyperpolarization-activated cyclic nucleotide-gated (HCN) c) Ether à go-go (EAG) family. CNG channels are voltage insensitive and non-selective cation channels that play a role in sensory systems (olfactory, visual). They are classified under $\mathrm{K}_{\mathrm{v}}$ channels due to similarity in structural features, not due to their ability to sense voltage. HCN channels gating is voltage-dependent and cyclic nucleotide sensitive. In response to hyperpolarization, they allow a cation current that is weakly selective to $\mathrm{K}^{+}$over $\mathrm{Na}^{+}$. They depolarize the membrane in response to hyperpolarization, hence facilitating the 
following action potential. They are responsible for rhythmic firing in the heart (pacemaker in the sinoatrial node) (James and Zagotta, 2018).

EAG belongs to the EAG family $(K C N H)$ of $\mathrm{Kv}$ channels, which can be divided into three sub-families: EAG: Kv10.1 and Kv10.2, ERG (EAG-related gene): Kv11.1, Kv11.2, Kv11.3 and ELK (EAG like K channel): Kv12.1, Kv12.2, Kv12.3. The most notorious member is Kv11.1, due to its role during the cardiac action potential. The peculiar kinetics of Kv11.1 restricts $\mathrm{K}^{+}$efflux during the repolarizing phase of the cardiac action potential. Mutations in Kv11.1 result in cardiac arrhythmia and long QT syndrome. The Eag subfamily (Kv10.1 and Kv10.2) are well-known for their role in cell proliferation and tumorigenesis.

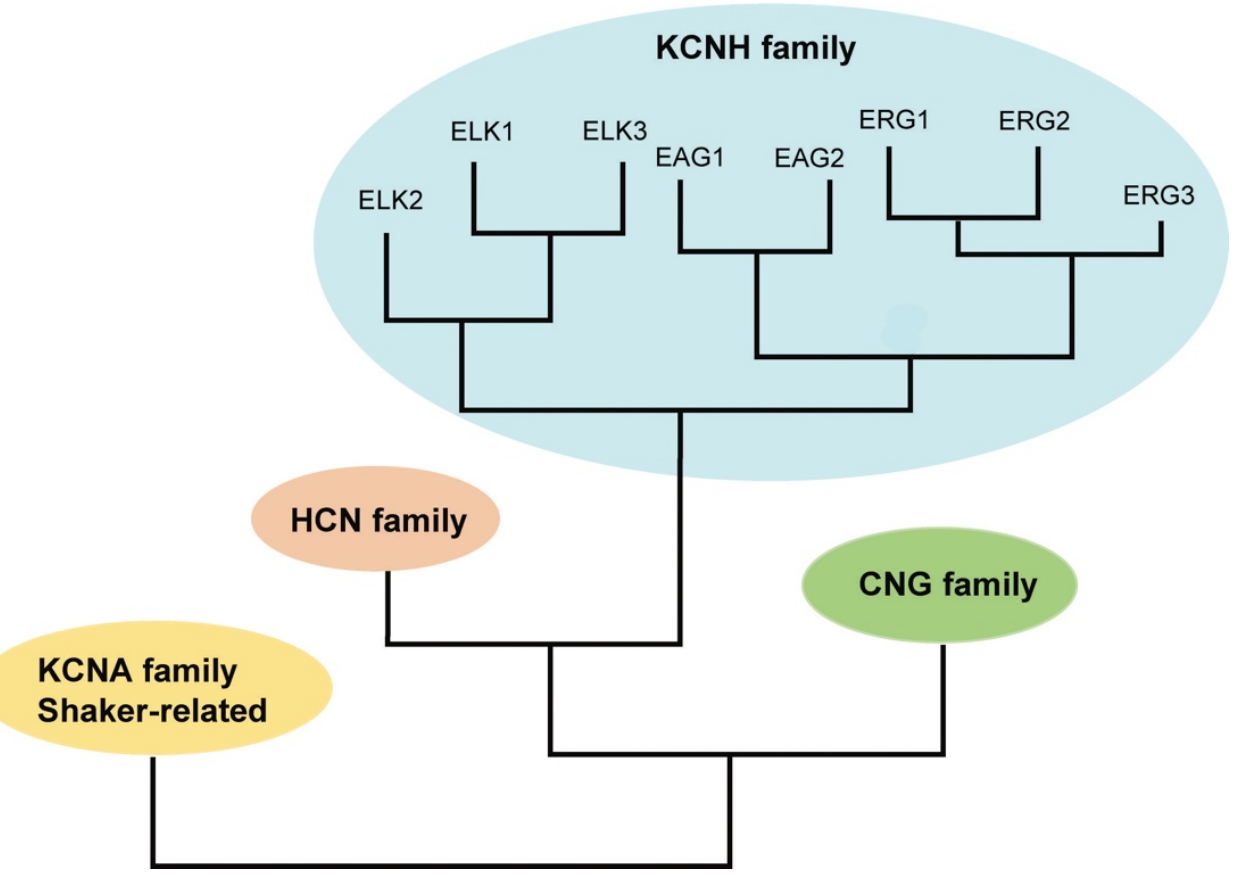

Figure 1. Cladogram of Voltage-gated $\mathbf{K}^{+}$channels $\left(\mathbf{K}_{\mathbf{v}}\right) \mathrm{HCN}$ and CNG are closer relatives to the KCNH than to the KCNA family. Adapted from (Codding et al., 2020) 


\subsection{Structural Features of KCNH (EAG) channels}

\subsubsection{Voltage sensor}

The S4 segment in the voltage-sensing domain (VSD) is the main component of the voltage sensor of voltage-gated channels; mutating positively charged residues (Arg (R) or Lys(K)) in S4 decreased voltage sensitivity (Stühmer et al., 1989). Mutations in Kv11.1 revealed that three (K1-R3) out of six positive charges are relevant for sensing voltage (Zhang et al., 2004), while shaker needs one more residue (R1-R4) (Aggarwal and MacKinnon, 1996). Two hydrophobic residues separate the charged ones. Hence, once the S4 segment adopts $\alpha$ helical conformation, the charged residues are aligned and interact with negative charges on the S2 segment. The negative charge on the S2 segment is called the charge-transfer segment; it plays a role in stabilizing the positive residues as they move in response to voltage stimuli.

The extent of movement of the sensor has been topic of long debate. The current consensus is that the S4 segment, which is tilted with respect to the rest of the voltage sensor, undergoes a rotation and a small displacement towards the extracellular side in response to depolarization. The displacement of the segment is probably smaller than previously hypothesized due to the focused electric field. The S4 segment is surrounded by hydrophilic cavities, which results in a smaller separation between intracellular and extracellular environment, hence a focused electric field (Sansom, 2000; Swartz, 2008).

\subsubsection{Pore}

The pore domain is formed by the S5-S6 segments and the P-loop in between. The pore comprises a selectivity filter, a central cavity, and an intracellular gate. The intracellular gate is formed close to the cytoplasmic end of S6, where the bundle of helices is constricted to close the gate. When helices move apart in response to a kink induced around a conserved Gly residue above the gate (Imbrici et al., 2009; Wang and MacKinnon, 2017), the gate is opened. In Shaker-like Kv channels, the activation gate 
is formed by a PVP sequence (del Camino et al., 2000). Proline induces a kink, which allows interaction with the S4-S5 linker. $K C N H$ channels lack the PVP motif; instead, the gate is constricted around a Gln residue (Thouta et al., 2014). The cryo-EM structure of rat Kv10.1 has revealed a closed conformation of the channel (Whicher and MacKinnon, 2016). The closed conformation captured shows straight S6 helices that constrict $(<1 \AA)$ around a Gln residue at position 476. A right-hand twist of the S6 helices keeps the gate shut (Whicher and MacKinnon, 2016). The Kv11.1 cryo-EM structure, which is in the open conformation, reveals a kink in the S6 helices around Gly648, which opens the gate residue (Gln664) (11 Å) (Wang and MacKinnon, 2017) (James and Zagotta, 2018).

The selectivity filter in $\mathrm{K}^{+}$channels preferentially accommodates $\mathrm{K}^{+}$over smaller monovalent cations like $\mathrm{Na}^{+}$. In $\mathrm{K}_{\mathrm{v}} 1-\mathrm{K}_{\mathrm{v}}$ 9, the filter is aligned with the backbone carbonyls of the stretch TVGYG in the P-loop. In KCNH channels, the conventional GYGD is replaced with GFGN (Chen et al., 2014). The oxygen molecules of the carbonyl groups can cage $\mathrm{K}^{+}$ions, replacing water molecules of their hydration shell. The low energy state of $\mathrm{K}^{+}$inside the pore compensates for the energy cost of the dehydration process. $\mathrm{K}^{+}$ions can bind to four sites in the selectivity filter (S1-S4); however, they occupy two sites at a time $(\mathrm{S} 1, \mathrm{~S} 3)$ or $(\mathrm{S} 2, \mathrm{~S} 4)$ due to the repulsion between $\mathrm{K}^{+}$ions (Kuang et al., 2015). $\mathrm{K}^{+}$ions can flow from one side to the other via a knock-on mechanism; one $\mathrm{K}^{+}$ ion enters from one side and knocks on the following one (Kopec et al., 2018). The conduction of $\mathrm{K}^{+}$is quite efficient and occurs at a rate near the diffusion limit. The pore is constitutively filled with $\mathrm{K}^{+}$ions. In the absence of $\mathrm{K}^{+}$, the channel can transiently conduct $\mathrm{Na}^{+}$, but the pore eventually collapses irreversibly (Krishnan et al., 2005; Kuang et al., 2015; Lockless et al., 2007; Zhou et al., 2001).

\subsubsection{Intracellular domains}

The $K C N H$ family share interesting intracellular domains that sets them apart from other $\mathrm{K}_{\mathrm{v}}$ channel families: the eag domain at the $\mathrm{N}$-terminus and a cyclic nucleotide-binding homology domain (CNBHD) at the C-terminus. The eag domain is alternatively known as the PAS domain. PAS is an acronym that combines the proteins where the sequence was initially recognized (PER-ARNT-SIM). The PAS domain is 
conserved across the different kingdoms. It can be found in other proteins that sense light, oxygen, and the cell energy level (Taylor and Zhulin, 1999). It is formed of six central $\beta$-sheets, surrounded by $\alpha$-helices. The first 25 residues are the least conserved; they form a helix and an extended loop called the PASCap domain (Pellequer et al., 1998).

The cyclic nucleotide-binding homology domain (CNBHD) is very similar to the cyclic nucleotide-binding domains (CNBD) of HCN and CNG channels. CNBD and CNBHD share a general architecture: a central $\beta$-roll (eight $\beta$-sheets) flanked by three $\alpha$-helices. Helix-A precedes the $\beta$-roll, while helices B and C follow the $\beta$-roll. The $\beta$-roll of CNBD has a binding pocket for cyclic nucleotides. Once cyclic nucleotides fill the pocket, helix-C functions as a cap (James and Zagotta, 2018). The pocket in CNBHD, on the contrary, doesn't bind cyclic nucleotides (Brelidze et al., 2009). It lost residues important for binding. The Arg residue that binds the cyclic phosphate (Arg549 in HCN1) (Zhou and Siegelbaum, 2007) is replaced by Ser (Ser630) in rat $K_{v} 10.1$. The pocket is filled instead with a short loop that follows the $\mathrm{C} \alpha$-helix. Three residues (Tyr (Phe)-Asn-Leu) form the loop, known as the intrinsic ligand (IL). CNBD and CNBHD are connected to the channel gate via the C-linker, which is composed of $\alpha$-helices: $A^{\prime}, B^{\prime}$, C', D', E' and F' (James and Zagotta, 2018). 


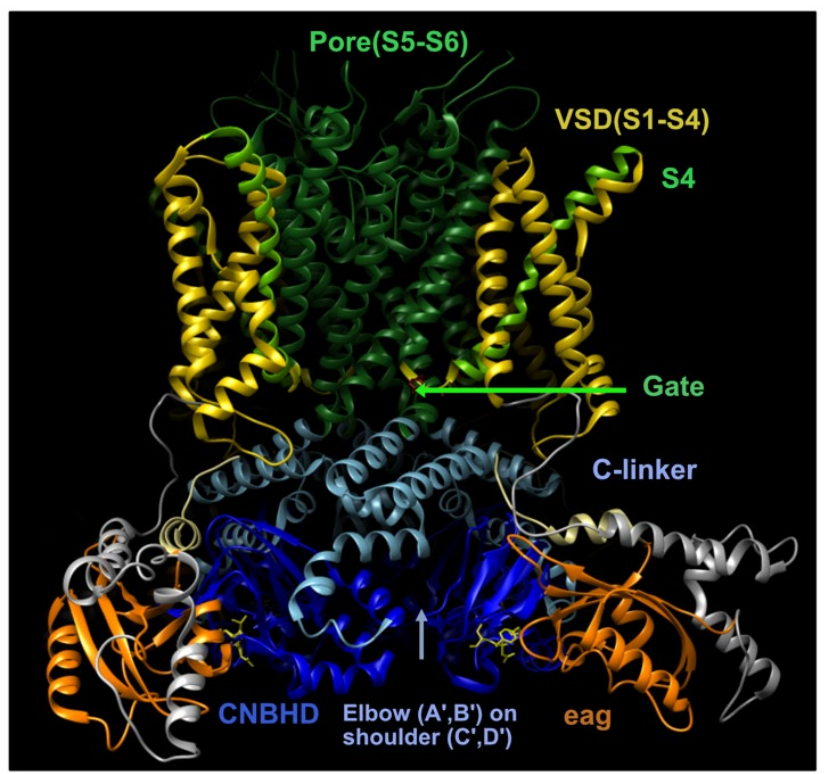

Figure 2. Cryo-EM structure of rat $K_{v} 10.1$ (Whicher and MacKinnon, 2016). Closed conformation of rat Kv10.1. The channel is bound to $\mathrm{Ca}^{2+}$ Calmodulin, which is not shown in the Figure to visualize the intracellular domains. The C-terminal domain (CNBHD) in blue is connected to the gate via the C-linker (sky blue). The eag domain (N-terminal domain) of a neighboring subunit interacts with CNBHD.

The eag and CNBHD domains form an intracellular ring connected to the channel core via the $\mathrm{C}$-linker. The eag domain of one subunit is located beneath the VSD of the same subunit and interacts with the CNBHD from a neighboring subunit. This arrangement where domains of different subunits interact with each other compensates for the conventional domain swapping in $\mathrm{K}_{\mathrm{v}}$ channels, where the voltage sensor of one subunit interacts with the pore domain of an adjacent subunit. $K C N H$, HCN, and CNG channels all lack the classical domain swapping and instead swap their intracellular domains (Codding et al., 2020). In addition to the eag - CNBHD interaction, inter-subunit interaction occurs through the C-linker; A', B' $\alpha$-helices lie on top of C', D' $\alpha$-helices from a neighboring subunit (elbow-on-shoulder) (James and Zagotta, 2018). In the case of Eag channels, opposing subunits can be additionally bridged by $\mathrm{Ca}^{2+}$ Calmodulin.

The C-linker is connected to the channel gate at the bottom of the S6 helices. The gate is formed by a right-hand twist of the helices at the bottom of S6 (see chapter 1.4.2.). A rotation of the $\mathrm{C}$-linker assembly can oppose the helices twist at the gate. $\mathrm{A}$ counter-clockwise rotation in the ring has indeed been observed when comparing the 
cryo-EM structures of Kv11.1 (which is in the open conformation) and rat $\mathrm{K}_{\mathrm{v}} 10.1$ (closed conformation) (James and Zagotta, 2018; Wang and MacKinnon, 2017; Whicher and MacKinnon, 2016). The rotation in the ring could be transmitted to the $C$ linker through the CNBHD, similar to the mechanism proposed for the CNBD in CNG and HCN (James and Zagotta, 2018; Wang and MacKinnon, 2017; Whicher and MacKinnon, 2016), where cyclic nucleotides bind to the $\beta$-roll of the CNBD causing a rotation of helices-B and C. Consequently, a force is applied on the C-linker through two of its helices (E', F') causing its rotation (Fig. 2). As stated above, in $K C N H$ channels the CNBHD does not bind cyclic nucleotides and the IL instead occupies its pocket. Nevertheless, the cryo-EM structure suggests that $K C N H$ and $H C N$ channels rotate in a similar way (James and Zagotta, 2018; Wang and MacKinnon, 2017; Whicher and MacKinnon, 2016).

What triggers the rotation of the intracellular domains in these channels is still an open question. It is plausible that the movement of the sensor is coupled to the rotation of the ring. Patch-Clamp fluorometry (PCF) experiments using tmFRET reported a change in the distance between eag and CNBHD domains of $\mathrm{K}_{\mathrm{v}} 12$ channels during voltage-dependent gating (Dai and Zagotta, 2017). Moreover, the dynamic movement of the intrinsic ligand in response to voltage has been observed with FRET experiments (Dai et al., 2018). These observations support the possibility of coupling the S4 movement to a rearrangement -possibly rotation- of the ring. The exact mechanism is still unclear; however, the PASCap is likely involved. The cryo-EM structure of rat $\mathrm{K}_{\mathrm{v}} 10.1$ highlights the critical location of the N-terminus (Whicher and MacKinnon, 2016). The initial N-terminus segment is directed toward a cavity close to the bottom of the S4 segment, S4-S5 linker, and C-linker. These are all molecular players important in voltage-dependent gating of the channel. A recent study proposed a possible interaction between the initial N-terminus (R7AR8A) and the bottom of S4 (D342) that stabilizes an open state. The authors suggest that a different set of interactions between the lower end of S4 or S4-S5 and the N-terminus could occur (Whicher and MacKinnon, 2019). 
a

Non-Domain-Swapped

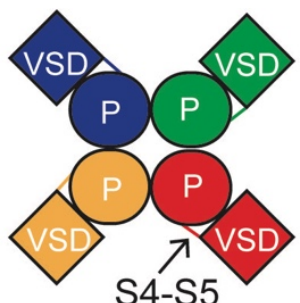

Linker

$\mathrm{KCNH}$ Channels b Domain-Swapped

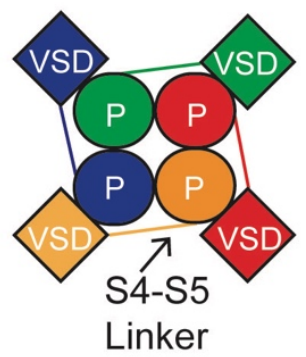

Other Kv Channels

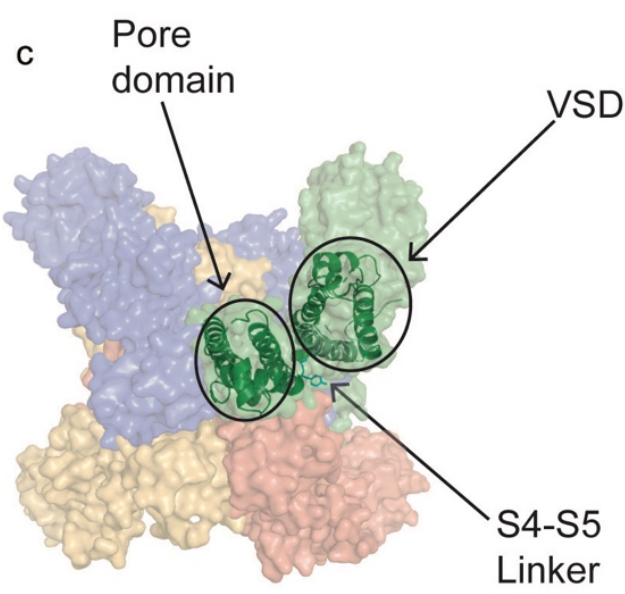

hERG (KCNH2)

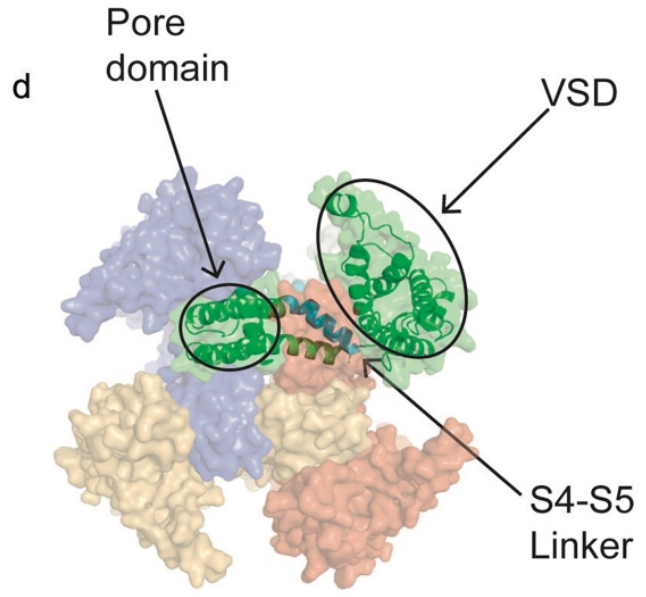

Kv 1.2

Figure 3. Domain swapping of S4-S5 linker. A) Cartoon that depicts non-domain swapping in KCNH channels B) Cartoon that depicts domain swapping in shaker related channels ( $\left.K_{v} 1-K_{v} 9\right)$. C) Structure of Kv11.1 d) Structure of Kv1.2. Adapted from (Codding et al., 2020)

Disturbing the integrity of the intracellular ring alters the gating kinetics of different members of the $K C N H$ family. The surface of interaction between eag and CNBHD domains plays a fundamental role in maintaining the ring structure. A point mutation breaking a salt bridge between the two domains is sufficient to alter the channel gating properties: R57D, D642R in mouse $\mathrm{K}_{\mathrm{v}} 10.1$ (Haitin et al., 2013), R56D, D803R in Kv11.1 (Ng et al., 2011) and R57D, D681R in Kv12 (Dai and Zagotta, 2017). Another point mutation (E627R) at the interface between the PASCap and the CNBHD shifts the conductance-voltage relationship (GV) to more depolarized potentials in mouse $\mathrm{K}_{\mathrm{v}} 10.1$ channels (Haitin et al., 2013). 
The intrinsic ligand (IL) is critical for maintaining eag-CNBHD interaction. In $\mathrm{K}_{\mathrm{v}} 11.1$, a mutation of the intrinsic ligand (F860A) interferes with the eag-CNBHD interaction, as demonstrated by FRET and gating assays. It was suggested that the ligand acts allosterically and ensures that the CNBHD adopts a conformation that can interact with the PAS domain (Codding and Trudeau, 2019). However, the crystal structure of mouse $\mathrm{K}_{\mathrm{v}} 10.1$ point to an interaction between the PAS domain and the IL (Haitin et al., 2013), and the cryo-EM structures of rat $\mathrm{K}_{\mathrm{v}} 10.1$ and Kv11.1 show the PAS domain and IL in proximity (Wang and MacKinnon, 2017; Whicher and MacKinnon, 2019). Whether the ligand acts directly or allosterically, there is a consensus that it is crucial for the interaction between eag and CNBHD.

Disturbing the ring alters different biophysical properties of $K C N H$ subfamilies: Cole-Moore in Kv10.1, voltage-dependent potentiation (VDP) in $\mathrm{K}_{\mathrm{v}} 12$, and mode shift and deactivation kinetics in $\mathrm{K}_{\mathrm{v}} 11.1$. Kv10 channel activation is delayed (Cole-Moore) and slowed down by hyperpolarizing pre-pulse (see chapter 1.5.1.2) (Hoshi and Armstrong, 2015). This behavior is lost when IL is mutated, or CNBHD is deleted (Zhao et al., 2017). The N-terminus deletions also compromised the Cole-Moore effect (Terlau et al., 1997; Whicher and MacKinnon, 2019). $\mathrm{K}_{\mathrm{v}} 12$ is characterized by VDP, which consists of a shift of the GV to the left, potentiation of the current amplitude, and slowing down the deactivation after positive pre-pulses. The kinetic features of VDP can be explained as a transition to a stable open state (termed Os) from a metastable state $(\mathrm{Om})$. A rearrangement in the ring (eag-CNBHD) seems to mediate the transition into Os (Dai and Zagotta, 2017). Deleting or mutating the intrinsic ligand of $\mathrm{K}_{\mathrm{v}} 12$ perturbed the characteristic VDP (Dai et al., 2018). In $\mathrm{K}_{\mathrm{v}} 11.1$, mode shift, a hyperpolarizing shift of the GV in response to positive pre-pulses, is intrinsic to the sensor and coupled to the gate through the N-terminus (Tan et al., 2012). The Nterminus could stabilize the sensor, slowing its return to the resting position, hence attenuating the mode shift (Goodchild et al., 2015). The N-terminus also plays a role in the characteristic slow deactivation of Kv11.1; eag deletion accelerates deactivation (Gustina and Trudeau, 2012; Ng et al., 2011; Wang et al., 2000; Wang et al., 1998). The $\mathrm{N}$-terminus likely slows down activation through stabilizing an open state (Goodchild et al., 2015; Robertson and Morais-Cabral, 2020). Interaction of eag domain and 
CNBHD is required to slow down deactivation (Gustina and Trudeau, 2011). Disturbing their interaction through IL mutations results in fast deactivation kinetics (Codding and Trudeau, 2019). In summary, the ring seems to stabilize an open state across the three KCNH families (Robertson and Morais-Cabral, 2020)

Small molecules targeting the ring can influence channel gating. Flavonoids can mimic the intrinsic ligand and potentiate voltage-dependent gating of $K_{v} 10.1$. Flavonoid binding to CNBHD shifts the voltage dependence to the left (Carlson et al., 2013). Chlorpromazine was recently shown to bind to the PAS domain and inhibit KCNH channels (mouse $\mathrm{K}_{\mathrm{v}} 10.1$ and $\mathrm{K}_{\mathrm{v}} 11.1$ ) (Wang et al., 2020). These molecules highlight the significance of these domains as potential targets to modify channel gating.

In addition to the main intracellular domains discussed above, a coiled-coil domain for tetrameric assembly (Jenke et al., 2003) and a ciliary localization signal are present at the C-terminus (Sánchez et al., 2016).

\section{$1.5 \mathrm{~K}_{\mathrm{v}} 10.1$}

The EAG (Ether à go-go) mutation was first recognized in a Drosophila melanogaster screening; it gained its nomenclature from the phenotype it presented a leg-shaking similar to a go-go dance - (Kaplan and Trout, 1969; Warmke et al., 1991). Eag is encoded by the KCNH1 gene (HUGO nomenclature) (Gutman et al., 2005). The channel has at least four isoforms arising from alternative splicing: the most abundant isoform (Eag1a), a longer isoform (Eag1b) with 27 residues extra from exon 6, and two short isoforms E65 and E70. Eag1a and Eag1b have similar biophysical behavior. E65 and E70 lack the transmembrane segments, and therefore they do not assemble into a functional channel. However, when co-expressed with full-length (Eag1a), they inhibit the current (Ramos Gomes et al., 2015). In this study we used the most abundant isoform Eag1a, Kv10.1 in the IUPAR nomenclature. 
$\mathrm{K}_{\mathrm{v}} 10.1$ is almost exclusively expressed in the CNS. $\mathrm{K}_{\mathrm{v}} 10.1$ mRNA and protein were detected in the hippocampus, cerebellum, cerebral cortex, and olfactory bulb (Martin et al., 2008; Saganich et al., 2001). Its role in the CNS is not fully understood. A knockout mouse lacking the pore and the voltage sensor showed no major alterations in behavior or development (Ufartes et al., 2013). The mice only showed mild hyperactivity and prolonged haloperidol-induced catalepsy. However, in zebrafish, $\mathrm{K}_{\mathrm{v}} 10.1$ was reported to be critical for development (Stengel et al., 2012). The discrepancy can be attributed to the model organism or because the intracellular domains are intact in the $\mathrm{KO}$ mice. Recordings of $\mathrm{K}_{v} 10.1$-deficient mice reveal a role of $\mathrm{K}_{\mathrm{v}} 10.1$ at the presynaptic synapse of cerebellar neurons (Mortensen et al., 2015). $\mathrm{K}_{\mathrm{v}} 10.1$ limits the frequency of action potential firing through regulating $\mathrm{Ca}^{2+}$ level and neurotransmitter release at the presynaptic terminal. Gain of function mutations of $\mathrm{K}_{\mathrm{v}} 10.1$ have been linked to neurological diseases: Zimmermann-Laband and TempleBaraitser syndromes. ZLS and TBS patients suffer from epileptic seizures, intellectual disability, along with facial deformities (Han et al., 2017).

Expression of $\mathrm{K}_{\mathrm{v}} 10.1$ outside the CNS is time-limited, which caused initial difficulty in detecting the channel in peripheral tissues. $K_{\mathrm{v}} 10.1$ is expressed during the G2/M phase of the cell cycle under the control of the E2F1 transcription factor (Urrego et al., 2016). Expressing a $\mathrm{K}^{+}$channel hyperpolarizes the cell, hence increasing the driving force for $\mathrm{Ca}^{2+}$ (Urrego, 2017). $\mathrm{K}_{\mathrm{v}} 10.1$ also interacts with Orai1 ( $\mathrm{Ca}^{2+}$ channel) and participates in the regulation of intracellular $\mathrm{Ca}^{2+}$ (Badaoui et al., 2018; Movsisyan and Pardo, 2020; Peretti et al., 2019). The enhancement of $\mathrm{Ca}^{2+}$ entry by hyperpolarization due to Kv10.1 activity could promote ciliary resorption (Urrego, 2017). Ciliary resorption is required to progress beyond the $G 2 / M$ transition. In addition to ciliary resorption, $\mathrm{K}_{\mathrm{v}} 10.1$ plays additional roles at the spindle assembly checkpoint of the cell-cycle; it decreases the expression of checkpoint proteins and alters microtubule dynamics and mitotic spindle assembly during mitosis (Movsisyan and Pardo, 2020). In summary, $\mathrm{K}_{\mathrm{v}} 10.1$ facilitates $\mathrm{G} 2 / \mathrm{M}$ transition and enhances proliferation.

Pathological disorders related to $\mathrm{K}_{\mathrm{v}} 10.1$ are indeed linked to aberrant proliferation; gain of function mutations can result into developmental disorders: 
Zimmermann-Laband and Temple- Baraitser syndromes, while sustained expression leads to tumorigenesis_(Cázares-Ordoñez and Pardo, 2015; Sánchez et al., 2016). $\mathrm{K}_{v} 10.1$ serves as a tumor marker and is found in $70 \%$ of human tumors. The restriction of $\mathrm{K}_{\mathrm{v}} 10.1$ expression to the CNS has made it a promising target for anti-cancer drugs. Antibodies targeting $\mathrm{K}_{\mathrm{v}} 10.1$ were used to deliver agents to induce apoptosis in tumor models and cancer cell-lines (reviewed in (Hernandez-Resendiz et al., 2019)).

\subsubsection{Biophysical properties}

\subsubsection{Inactivation}

Inactivation in $\mathrm{K}_{\mathrm{v}} 10.1$ varies among species; recordings of Drosophila $\mathrm{K}_{\mathrm{v}} 10.1$ reveal partly inactivating current (Bruggemann et al., 1993), while mammalian EAG mouse $\mathrm{K}_{\mathrm{v}} 10.1$ (Robertson et al., 1996) and rat $\mathrm{K}_{\mathrm{v}} 10.1$ (Ludwig et al., 1994) are characterized by non-inactivating current. Human $\mathrm{K}_{\mathrm{v}} 10.1$ has been reported to have slow, subtle inactivation (5-10\% reduction in current). This intrinsic inactivation could be enhanced by a small molecule (3-nitro-N-(4-phenoxyphenyl) benzamide (ICA105574)) and a mutation in the S6 segment (Y464A). The loss of the aromatic ring of Y464 reveals intrinsic inactivation, which can be rescued by mutating one of the paired residues in S5 (F359A) or (1434A) in the pore helix. The aromatic residue of Y464A stabilizes the aromatic and hydrophobic residues of (F359 - 1434) when lost inactivation is revealed. These three residues are suggested to be a molecular triad responsible for inactivation in $\mathrm{K}_{\mathrm{v}} 10.1$. A small molecule (ICA105574) has also been reported to enhance intrinsic inactivation, possibly through this triad (Garg et al., 2012).

\subsubsection{Cole-Moore}

The activation kinetics of the $K C N H$ family can be modulated by hyperpolarized pre-pulses and cations such as $\mathrm{Mg}^{2+}$. Hyperpolarized pre-potentials cause a delay of the activation of $\mathrm{K}^{+}$channels in squid axons (Cole and Moore, 1960), resulting in sigmoidal activation. This phenomenon is commonly termed "Cole-Moore effect/Shift" and can 
be explained by the five-state model of Hodgkin and Huxley (C01-C-C-C-O); the hyperpolarized potentials trap the channels in deep closed states (Co) hence delaying the activation as the channel needs to transition among more closed states (C0-C1-C3C4) before reaching the open state (0). In a classical Cole-Moore shift, the last activation step (C-O) shares the same kinetics; hence the current responses with different pre-pulse potentials can be shifted to compensate for the delay and superimposed. The classical Cole-Moore shift can be observed in Shaker and Kv1 channels, which share similar kinetics with the squid axons' channels. However, Kv10.1 show a similar but distinct phenomenon from the classical Cole-Moore shift. In response to hyperpolarized pre-pulses, the sigmoidal activation is observed; however, the delay is in the range of tens of milliseconds. More importantly, the current traces are non-superimposable. Moreover, $\mathrm{Mg}^{2+}$ can further enhance the sigmoidal shape of the current (Hoshi and Armstrong, 2015). We will nevertheless refer to the induction of a sigmoid and slowed activation by hyperpolarization as Cole-Moore shift, to stick to the usual terminology in the field.

\subsubsection{Regulation of $\mathrm{K}_{\mathrm{v}} \mathbf{1 0 . 1}$}

A variety of molecules regulate Kv10.1 (reviewed in (Han et al., 2017)). Its membrane expression can be modulated by Epsin, Rabaptin-5, CaM-Kinase II, cortacin and glycosylation (Han et al., 2016; Herrmann et al., 2012; Napp et al., 2005; Ninkovic et al., 2012; Piros et al., 1999; Sun et al., 2004). Epsin is a protein involved in endocytosis (Piros et al., 1999), while Rabaptin-5 interacts with endocytic (Rab5) and recycling proteins (Rab4) (Ninkovic et al., 2012). Cortactin is proposed to limit the endocytosis of $\mathrm{K}_{\mathrm{v}} 10.1$ via an interaction with actin filaments (Herrmann et al., 2012). $\mathrm{K}_{\mathrm{v}} 10.1$ function can also be modulated. Cys modification by $\mathrm{H}_{2} \mathrm{O}_{2}$ can alter its gating kinetics and inhibit the current. Phosphatidylinositol (4-5) bisphosphate (PIP2) and $\mathrm{Ca}^{2+}$-Calmodulin (CaM) inhibit the channel (Han et al., 2016; Schönherr et al., 2000); they both share a binding site at the N-terminus which might influence eag-CNBHD interaction. Extracellular divalent cations $\left(\mathrm{Mg}^{2+}\right)$ slow down the movement of the S4 segment (the sensor), causing sigmoidal activation (Han et al., 2017). We would like to focus on the effect of $\mathrm{Ca}^{2+}-\mathrm{CaM}$ and $\mathrm{Mg}^{2+}$ on $\mathrm{K}_{\mathrm{v}} 10.1$ kinetics. 
The modulation of Drosophila Kv10.1 kinetics by $\mathrm{Mg}^{2+}$ characterizes the channel and distinguishes it even from its close relative ERG (Schönherr et al., 1999; Terlau et al., 1996). $\mathrm{Mg}^{2+}$ binds to negative charges (D278 and D327) in the S2-S3 transmembrane segment shielding the negative charges and stabilizing a deep closed conformation (Silverman et al., 2000). $\mathrm{Mg}^{2+}$ binding narrows the gating canal and restricts the outward movement of the sensor (Schönherr et al., 2002). $\mathrm{Mg}^{2+}$ slows down the transitions among deep closed states (Cs), increasing the sigmoidicity of activation. It also alters the pre-pulse dependence; stronger depolarized pre-pulses are required to accelerate activation (Terlau et al., 1996). However, the last step of activation (C-O) is $\mathrm{Mg}^{2+}$-independent (Schönherr et al., 2002; Terlau et al., 1996), consequently the deactivation kinetics (O-C) is not altered by $\mathrm{Mg}^{2+}$ binding (Terlau et al., 1996). Other cations like $\mathrm{Mn}^{2+}$ and $\mathrm{Ni}^{2+}$ bind to the same cavity (S2-S3) and slow activation similar to the effect of $\mathrm{Mg}^{2+}$ (Silverman et al., 2004).

\subsubsection{Calmodulin}

Calmodulin ( $\mathrm{CaM})$ is an abundant soluble protein $(0.5 \%$ of total cell protein content). It is found in the nucleus, cytoplasm, and near the plasma membrane (Saimi and Kung, 2002). It is an important protein in cell signaling and cell physiology; it is involved in several many processes; apoptosis, muscle contraction, NO synthesis, cyclic nucleotide synthesis, phosphorylation (CaMkinase CamK), and dephosphorylation (Calcineurin) (James et al., 1995). CaM is a 148-residue (17 Kd) peptide highly conserved among the eukaryotic families (Saimi and Kung, 2002). CaM consists of two lobes at each end of the peptide (N- and C-lobe) with a flexible helix in between. Each lobe has two EF-hands (N-lobe: EF1,2; C-lobe: EF3,4); negative charges in each EF-hand can form coordinate bonds with a $\mathrm{Ca}^{2+}$ ion; thus, one lobe is occupied with two $\mathrm{Ca}^{2+}$ ions. The affinity of each lobe to $\mathrm{Ca}^{2+}$ is different; the C-lobe has higher affinity ( $\mathrm{Kd}=100$ $\mathrm{nM})$ in comparison to the $\mathrm{N}$-lobe $(\mathrm{Kd}=1 \mu \mathrm{M})$ (James et al., 1995). The protein affinities to $\mathrm{Ca}^{2+}$ in isolation change when $\mathrm{CaM}$ interacts with the target protein (Bayley et al., 1996; Stefan et al., 2008). The difference in affinity of the two lobes leads to an intermediate state with only the $\mathrm{C}$-lobe bound to $\mathrm{Ca}^{2+}$ while the $\mathrm{N}$-lobe is free; hence CaM can exist in three states $\left(\mathrm{Ca}^{2+}\right.$ free (apoCaM), $4 \mathrm{Ca}^{2+}$ bound (holoCaM), $2 \mathrm{Ca}^{2+}$ bound) (Saimi and Kung, 2002). Once $\mathrm{Ca}^{2+}$ fills the E2F hands, a hydrophobic patch is 
exposed which mediates the interaction with the target protein (Komeiji et al., 2002). The kinetics of the conformational change at each lobe is different; the C-lobe is faster $(490 \mu \mathrm{s})$ than the N-lobe $(20 \mathrm{~ms})$ (Park et al., 2008). CaM has a flexible conformation; it can adopt an extended or a compact form. The binding of both $\mathrm{Ca}^{2+}$ and the target peptide alters CaM conformation (Kawasaki et al., 2019).

CaM binds to a wide variety of proteins, either in a constitutive or $\mathrm{Ca}^{2+}$ dependent manner. CaM often but not exclusively binds to calmodulin-binding motifs; the IQ motif mediates $\mathrm{Ca}^{2+}$-independent binding, while 1-8-14 and 1-5-10 motifs bind to $\mathrm{Ca}^{2+}-\mathrm{CaM}$. The motifs comprise alternating basic and hydrophobic residues; the numbers refer to conserved hydrophobic residues. For some proteins like myosin, both modes of binding are detected; myosin chains bind CaM constitutively but need $\mathrm{Ca}^{2+}$ CaM for a stronger interaction (Rhoads and Friedberg, 2016). Constitutively bound CaM can help ion channels sense $\mathrm{Ca}^{2+}$ fast (on a scale of ms) resulting in opening (ex: SK channels) or inhibition (ex: L-type $\mathrm{Ca}^{2+}$ channels) of the channel. In SK channels, $\mathrm{CaM}$ is bound to a monomer of the channel via the $\mathrm{C}$-lobe $\left(\mathrm{Ca}^{2+}\right.$ free). Once $\mathrm{Ca}^{2+}$ fills the N-lobe, the monomers dimerize to have an open SK channel (Saimi and Kung, 2002). In L-type $\mathrm{Ca}^{2+}$ channels, $\mathrm{CaM}$ is bound to an IQ-like motif at the C-terminus. $\mathrm{Ca}^{2+}-\mathrm{CaM}^{-}$ promotes inactivation of L-type $\mathrm{Ca}^{2+}$ channels, functioning as negative feedback (Peterson et al., 1999).In a traditional view, $\mathrm{Ca}^{2+}-\mathrm{CaM}$ binds to the channel first via the C-lobe (the lobe with higher affinity), then the N-lobe follows. The interaction is mediated mainly through hydrophobic residues, but electrostatic interaction is also possible; glutamate in the EF-hand can interact with basic residues in binding motifs. Secondary interactions should not be overlooked; in SK channels, a protrusion in the $\mathrm{N}$-lobe interacts with the $\alpha$-chain of the channel (Saimi and Kung, 2002).

$\mathrm{Ca}^{2+-} \mathrm{CaM}$ inhibits $\mathrm{K}_{\mathrm{v}} 10.1$ with an IC50 of $100 \mathrm{nM}$; channel inhibition was observed in inside-out patches treated with CaM protein in the presence of $\mathrm{Ca}^{2+}$ (Schönherr et al., 2000). CaM binds to typical $\mathrm{Ca}^{2+}$-CaM binding motifs $(1,8,14$ motif and 1,5,10 motif); one site at the N-terminus: binding domain-N (BD-N:151-165), and two sites at the C-terminus: binding domain-C1 (BD-C1: 1674-683) and binding domain-C2 (BD-C2:711-721) (Ziechner et al., 2006). The binding of CaM to $\mathrm{K}_{\mathrm{v}} 10.1$ is reportedly $\mathrm{Ca}^{2+}$-dependent. It is worthy to note that experiments were performed 
using peptide fragments of the channel (Goncalves and Stuhmer, 2010; MarquesCarvalho et al., 2016; Schönherr et al., 2000; Ziechner et al., 2006). Mutations at these binding sites disrupt $\mathrm{Ca}^{2+}-\mathrm{CaM}$ inhibition of $\mathrm{K}_{\mathrm{v}} 10.1$ : F151N.L154N at BD-N, R677N.R681N.K682N at BD-C1 and F714.F717S mutations at BD-C2 (Schönherr et al., 2000; Ziechner et al., 2006). BD-N and BD-C2 are more critical for binding than BD-C1 (Goncalves and Stuhmer, 2010; Ziechner et al., 2006). BD-C1, however, might influence the interaction between eag and CNBHD domains (Marques-Carvalho et al., 2016). In the resolved rat $\mathrm{K}_{\mathrm{v}} 10.1$ cryo-EM structure, BD-C1 and BD-C2 are located after the CNBHD, while BD-N is located after the eag domain (Whicher and MacKinnon, 2016). $\mathrm{BD}-\mathrm{N}$ binds to the N-lobe of $\mathrm{CaM}$, while the $\mathrm{C}$-lobe binds to BD-C1 and BD-C2 of an opposite subunit. The cryo-EM structure captured a snapshot of $\mathrm{K}_{\mathrm{v}} 10.1$ bound to $\mathrm{Ca}^{2+}$ $\mathrm{CaM}$, which closes the channel gate. It is however plausible that this is not the only binding mode of CaM to Kv10.1.

The binding of $\mathrm{Ca}^{2+}-\mathrm{CaM}$ to the intracellular domains closes the channel gate independently of the voltage stimulus (Lorinczi et al., 2016; Whicher and MacKinnon, 2016). A comparison of the open and closed conformations captured by Kv11.1 and rat Kv10.1 cryo-EM structures reveals a counterclockwise rotation (seen from the extracellular side) in the ring that dilates the packed helices at the gate. $\mathrm{Ca}^{2+}{ }_{-} \mathrm{CaM}^{-}$ bound to rat Kv10.1 may cross-bridge opposite subunits, hindering the rotation of the ring and therefore stabilizing a closed conformation (Wang and MacKinnon, 2017; Whicher and MacKinnon, 2016, 2019). This mechanism of global change in the ring seems highly plausible; however, an alternative mechanism has been proposed where local changes at BD-C2 could be sufficient to inhibit the channel (Marques-Carvalho et al., 2016). 


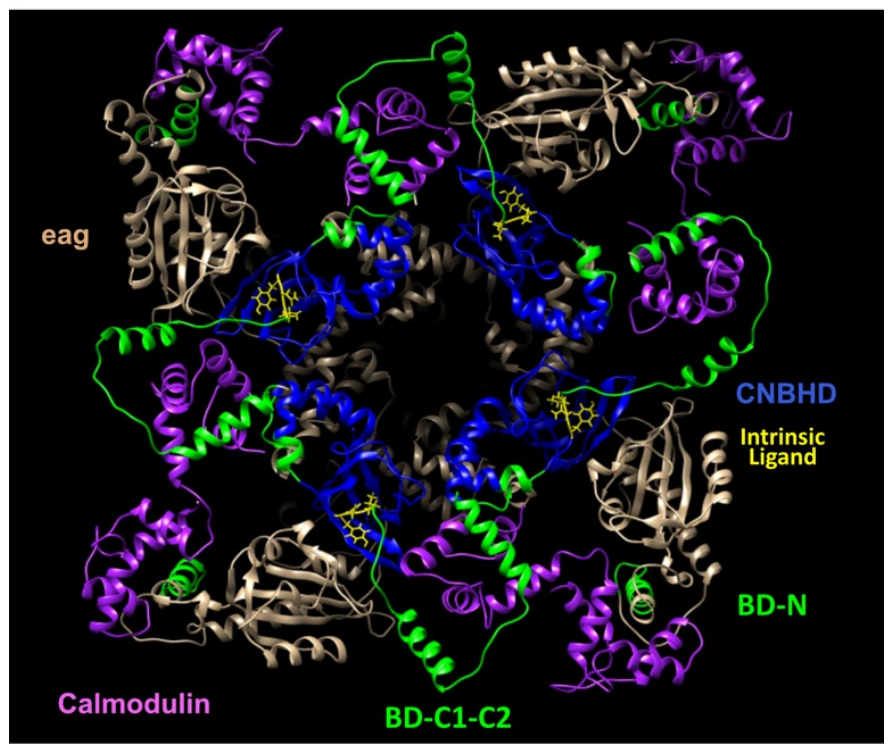

Figure 4. Bottom view of the cryo-EM structure of rat Kv10.1(Whicher and MacKinnon, 2016). CaM is shown in purple bound to $K_{v} 10.1$ at C-terminal sites (BD$\mathrm{C} 1$ and $\mathrm{BD}-\mathrm{C} 2)$ and the N-terminal site (BD-N). In green, Kv10.1 interaction surface with CaM. CaM binds close to intracellular domains (CNBHD in blue, intrinsic ligand in yellow, and eag in khaki)

\subsection{Modeling of voltage-gated ion channels}

\section{Two-State model}

The majority of voltage gated ion channels opens the gate in response to a depolarizing signal and closes when the membrane is repolarized. To model the conformational changes occurring, we assume a two-state model:

$$
C \underset{\beta}{\stackrel{\alpha}{\rightleftharpoons}} 0
$$

The channel transitions from a closed conformation (C) to an open one (O) with a rate constant $(\alpha)$ and goes back with the rate constant $(\beta)$. The transition requires energy which is provided by the voltage stimulus:

$$
E=z F V
$$

$z$ is the apparent charge valence; $F$ is Faraday constant and $V$ is voltage. 
The reaction rates $\alpha$ and $\beta$ are dependent on energy and hence the voltage stimulus applied. The stimulus provides the channel with sufficient energy to cross the energy barrier between the two states (Transition state theory). Therefore, $\alpha$ and $\beta$ can be described with an exponential dependence on the activation energy, the energy provided to cross the barrier (Destexhe and Huguenard, 2010; Eyring, 1935)

$$
\alpha=\alpha_{o} e^{-\Delta G(V) / R T}
$$

$\Delta G$ (activation energy) is the energy difference between the initial state and intermediate state, which is a function of voltage, $R$ is the gas constant and $T$ is the absolute temperature.

The equation can be reformulated as a function of voltage.

$$
\begin{gathered}
\alpha=\alpha_{o} e^{-\gamma z F V / R T} \\
\beta=\beta_{o} e^{(1-\gamma) z F V / R T}
\end{gathered}
$$

$\gamma$ is a constant that represents the energy barrier, $z$ represents the gating charge, charge that moved in response to the voltage stimulus.

The exponential function assumes a continuous increase in rates. To reflect the saturation of the channel kinetics, other modifications were suggested. We will focus on the sigmoidal function to explain the saturation of the rate voltage dependence.

$$
\begin{gathered}
\alpha=\frac{\mathrm{A}}{1+e^{-\left(V_{m}-V_{h}\right) / K}} \\
\beta=\frac{\mathrm{A}}{1+e^{\left(V_{m}-V_{h}\right) / K}}
\end{gathered}
$$

$A$ is an asymptotic value, the maximum value that can be reached; $V_{m}$ is the membrane voltage, which can be controlled in voltage-clamp recordings (stimulus); $V_{h}$ is the voltage that elicits half the maximum response and $K$ represents the slope, $\mathrm{K}=\mathrm{RT} / \mathrm{zF}$. 
The change in rates in response to voltage changes the occupancies of the states $\mathrm{C}$ and 0 . The probability of being in one of the two states can be described using ordinary differential equations, known as Kolmogorov equations (Linaro and Giugliano, 2015)

$$
\begin{gathered}
\alpha \\
C \rightleftharpoons 0 \\
\beta \\
\frac{d}{d t} P_{o}(t)=\alpha P_{c}(t)-\beta P_{o}(t) \\
\frac{d}{d t} P_{c}(t)=\beta P_{o}(t)-\alpha P_{c}(t)
\end{gathered}
$$

$\mathrm{P}_{\mathrm{o}}$ is the probability of occupying the open state and $\mathrm{P}_{\mathrm{c}}$ is is the probability of occupying the closed state. The distribution of the channel population between the two states, influence the overall conductance $(G)$

$$
\mathrm{G}=\mathrm{P}_{\mathrm{o}} \gamma \mathrm{N}
$$

$\gamma$ is single-channel conductance, $\mathrm{P}_{\mathrm{o}}$. is the probability of the channel being open and $\mathrm{N}$ is total number of channels. The current $(I)$ measured using whole-cell recordings can therefore be described as follows:

$$
\mathrm{I}=\mathrm{G}\left(\mathrm{V}_{\mathrm{m}}-\mathrm{V}_{\mathrm{eq}}\right)
$$

$\mathrm{G}$ is the overall conductance, $\mathrm{V}_{\mathrm{m}}$ is the membrane voltage and $\mathrm{V}_{\text {eq }}$ is the equilibrium potential for the relevant ion. In this study, we perform whole cell-recordings. We infer the conductance from the current recordings as described in materials and methods. 


\section{Multistate model}

The conformational changes of voltage-gated ion channels are more complex than those two states. In reality, the channel transition between several closed, open and inactive states. The term "inactive state" refers to a state where the gate opens but the channel does not conduct current. Some of these transitions depend on the voltage stimulus, and others do not. To formulate a gating scheme that describes such complex kinetics, one can assume that the channel population occupies a set of discrete states. The transition from one state to the other depends on the current state; the channel is not influenced by the history of transitions. The representation of channel kinetics using these assumptions is known as Markov model (Destexhe and Huguenard, 2010; Linaro and Giugliano, 2015).

\section{Modular gating scheme}

Defining the states, the channel occupies as open and closed is another simplification of the conformational changes the channel goes through. Each module of the channel (sensor and pore) can occupy different states. The sensor can be at a depolarized or at a hyperpolarized position, the pore can be opened or closed. In the case of CNG channels, where the C-linker and the CNBD contribute to gating, more states need to be considered. The C-linker can be active or at rest, while the CNBD can be bound or unbound to cyclic nucleotides. These modules influence each other. A depolarized voltage sensor will increase the probability of occupying the open state. Cyclic nucleotide binding to CNG further increases the probability of opening (James and Zagotta, 2018).

\subsection{Aim}

In our study, we investigated the electromechanical coupling of $\mathrm{K}_{\mathrm{v}} 10.1$, which cannot be explained by existing models that apply to Kv1-Kv9 families, where the S4S5 linker can function as a mechanical lever. Although we focus on $\mathrm{K}_{\mathrm{v}} 10.1$, structural and functional studies have suggested that the $K C N H$ family and their relatives -CNG 
and HCN families-could share a similar gating mechanism (James and Zagotta, 2018). Domain-swapping through S4-S5 is not possible $K C N H$ channels, as the linker is too short (5 residues). Instead, $K C N H$ channels swap their intracellular domains (James and Zagotta, 2018; Wang and MacKinnon, 2017; Whicher and MacKinnon, 2016). Additionally, the structure of the gate itself is different for KCNH channels (del Camino et al., 2000; James and Zagotta, 2018; Thouta et al., 2014; Wang and MacKinnon, 2017). Furthermore, KCNH channels lacking a covalent connection in S4-S5 linker (Split channels) show voltage-dependent gating, which refutes the mechanical lever model (de la Peña et al., 2018; Lorinczi et al., 2015; Tomczak et al., 2017). The failure of the mechanical lever model aroused the need to explore an alternative gating mechanism for this family.

The first question was how the intracellular ring could be coupled to the voltage sensor. The N-terminus was the molecular candidate that could fulfill such a role. We, therefore, set out to test the role of the $\mathrm{N}$-terminus in the gating of $\mathrm{K}_{\mathrm{v}} 10.1$, by examining its proximity to other molecular players of the gating machinery. Then we performed a series of deletions: ( $\Delta 2-10, \triangle$ PASCap, $\Delta$ eag) and a point mutation (E600R) to disrupt its interaction with CNBDH (Haitin et al., 2013)Lorinczi, 2016 \#230\} and examined their effects on gating using two-electrode voltage clamp (TEVC).

We then turned our focus to the role CaM in the gating of $\mathrm{K}_{\mathrm{v}} 10.1$. CaM, as revealed by the cryo-EM structure (Whicher and MacKinnon, 2016), binds to two opposite subunits in the presence of micromolar $\mathrm{Ca}^{2+}$. The integration of CaM molecule within $\mathrm{K}_{\mathrm{v}} 10.1$ intracellular domains, led us to speculate that CaM may be bound at basal $\mathrm{Ca}^{2+}$ levels and participate of the normal gating process. We proposed that CaM could be bound to one subunit at basal $\mathrm{Ca}^{2+}$ level, then cross-bridge it to the opposite subunit when $\mathrm{Ca}^{2+}$ is raised. A combination of mutagenesis, biochemical and electrophysiological approaches allowed us to test a model where CaM is part of the intracellular gating ring 


\section{Methods}

\subsection{Construct Generation}

Constructs were designed using SeqBuilder Pro version 15 (DNASTAR). psGEM.Kv10.1 was used for deletions and point mutations. We will refer to psGEM.Kv10.1 as wild type "WT".

\subsubsection{Deletion mutants}

To generate a series of constructs with a deletion in the intracellular regions of $\mathrm{K}_{\mathrm{v}} 10.1$, we used the In-Fusion HD Cloning kit (Clontech (TaKaRA)). The initial constructs were designed by Adam Tomczak and Jorge Fernández-Trillo. For each construct we designed two primers, each of them with two regions: a 3' region that anneals to the template immediately up- or downstream of the sequence to be deleted, and a 5' that does not bind to the template but overlaps with the second primer (Table 1). The subsequent PCR amplification will then omit the sequence between the hybridization sites for the primers. The primers were synthesized by Metabion. We used Kv10.1 constructs in the oocyte expression vector pSGEM (Prof. M. Hollmann, Bochum University) as a template. The PCR reaction comprised: $12.5 \mu \mathrm{l}$ CloneAmp HiFi PCR Premix provided by the kit and $6 \mu \mathrm{l}$ of each primer (forward and reverse, $1 \mathrm{pmole} / \mu \mathrm{l}$ ) to have a total volume of $25 \mu \mathrm{l}$. The PCR product was generated using the following thermocycling conditions: $98^{\circ} \mathrm{C}$ for $10 \mathrm{~s}, 55^{\circ} \mathrm{C}$ for $10 \mathrm{~s}, 72^{\circ} \mathrm{C}$ for $30 \mathrm{~s}$ ( 35 cycles). The linear DNA product was then purified using NucleoSpin Gel and PCR Clean-up (MACHEREY-NAGEL), to prepare it for a ligation reaction that re-circularized the plasmid. Ligation was performed using $2 \mu \mathrm{l}$ of 5X In-Fusion HD Enzyme Premix, provided with the kit, and $8 \mu$ of the PCR product. The reaction mix was incubated for $15 \mathrm{~min}$ at $50^{\circ} \mathrm{C}$, and then kept on ice. $\Delta$ Eag.pLeics 71 from was a generous gift from $\mathrm{Dr}$. John Mitcheson, University of Leicester. 
Table 1. Primers for infusion cloning

\begin{tabular}{|c|c|}
\hline & pSGEM. $\Delta 2-10$ \\
\hline Template & pSGEM.K. 10.1 / N-L341 \\
\hline Primer forward & $5^{\prime}$ attcgatatcaagcttatggtggccctcaaaacacgtttct 3' \\
\hline \multirow[t]{2}{*}{ Primer reverse } & $5^{\prime}$ cggtatcgataagcttcagctggctccaaaaatgtctctct $3^{\prime}$ \\
\hline & pSGEM $\Delta$ PASCap $(\Delta 2-25)$ \\
\hline Template & pSGEM K 10.1 \\
\hline Primer forward & 5' gctgccgccaccatg aatgatactaattttgtgttggggaa 3' \\
\hline \multirow[t]{2}{*}{ Primer reverse } & 5' catggtggcggcagctcg 3' \\
\hline & pSGEM $\triangle$ CNBHD $(\Delta 525-697)$ \\
\hline Template & psGEM. Kv10.1. \\
\hline Primer forward & $5^{\prime}$ tccagaggcattgac aatgaggccccctgatcttgc $3^{\prime}$ \\
\hline \multirow[t]{2}{*}{ Primer reverse } & 5' gtcaatgcctctggacatggaccaa 3' \\
\hline & pcDNA3. $\triangle$ PASCap \\
\hline Template & pcDNA3. $\Delta 2-10$ \\
\hline Primer forward & 5' gatatcgccaccatgaatgatactaattttgtgttggggaa 3' \\
\hline Primer reverse & 5' catggtggcgatatcgaattcc 3' \\
\hline
\end{tabular}

\subsubsection{Quick Change mutagenesis (Site-specific mutation)}

We performed site-directed mutagenesis to obtain point mutations using the Quick Change II XL Site-Directed Mutagenesis Kit (Agilent Technologies). The primers were synthesized by Metabion, and designed to have one or more mismatched base pairs corresponding to the desired mutation using the online tool as recommended by the kit manufacturer: (https://www.agilent.com/store/primerDesignProgram.jsp). The primer pairs for site-specific mutagenesis are shown in Table 2 . The reaction mixture was set up as follows: 10ng of template, $125 \mathrm{ng}$ of each primer, $0.5 \mu \mathrm{l}$ dNTPs $(10 \mathrm{nM}), 2.5 \mu \mathrm{l}$ reaction buffer(10x), $1.5 \mu \mathrm{l}$ DMSO and $0.5 \mu \mathrm{l}$ of Pfu Ultra HF DNA polymerase, then diluted with distilled water $\left(\mathrm{dH}_{2} \mathrm{O}\right)$ to a volume of $25 \mu \mathrm{l}$. Thermocycling conditions $\left(95^{\circ} \mathrm{C}\right.$ for $5 \mathrm{~min}, 95^{\circ} \mathrm{C}$ for $1 \mathrm{~min}, 60^{\circ} \mathrm{C}$ for $1 \mathrm{~min}, 68^{\circ} \mathrm{C}$ for $7.20 \mathrm{~min}$ (18cycles), $68^{\circ} \mathrm{C}$ for $10 \mathrm{~min}$ ) (Mastercycler gradient, Eppendorf) was setup to amplify the plasmid with the point mutation as a linear DNA. The template was digested using DpnI (New England Biolabs), which cleaves methylated DNA and therefore eliminates the parental plasmid template, while the mutated PCR product is 
not digested. We received E600R.pLeics71 from Dr. John Mitcheson, University of Leicester.

Table 2. Primers for site directed mutagenesis

\begin{tabular}{|l|l|}
\hline & D342C \\
\hline Template & WT \\
\hline Primer forward & 5'-cgagtggcccgtaagctgtgccactacattgaatatgga-3' \\
\hline Primer reverse & 5'-tccatattcaatgtagtggcacagcttacgggccactcg-3' \\
\hline & R8C \\
\hline Template & WT \\
\hline Primer forward & 5'-ggctgggggcaggtgtggactagtggccc-3' \\
\hline Primer reverse & 5'-gggccactagtccacacctgccccagcc-3' \\
\hline & R8C.C214A \\
\hline Template & R8C \\
\hline Primer forward & 5'-gtggtcttaaaaacagcataatgtaagatgatgtgagggggagtct-3' \\
\hline Primer reverse & 5'-agactcccctcacatcatcttacattatgctgtttttaagaccac-3' \\
\hline & R8C.C640A / R8C.C640A.C575A \\
\hline Template & R8C / R8C.C575A \\
\hline Primer forward & 5'-cttgatcacatgcagatcagcgtaggtcaaggccctaaca-3' \\
\hline Primer reverse & 5'-tgttagggccttgacctacgctgatctgcatgtgatcaag-3' \\
\hline & R8C.C575A \\
\hline Template & R8C \\
\hline Primer forward & 5'-gtccctggggcagcgtgcaccgtctgg-3' \\
\hline Primer reverse & 5'-ccagacggtgcacgctgcccaggggac-3' \\
\hline & \\
\hline & $\Delta 2$-10F151NL154N / $\Delta$ PASCap F151NL154N \\
\hline Template & $\Delta 2$-10 / $\Delta$ PASCap psGEM \\
\hline Primer forward (F151N) & 5'-ctcttgtcagccgagcattcttccccagcctttac-3' \\
\hline Primer Reverse (F151N) & 5'-gtaaaggctgggggaagaatgctcggctgacaagag-3' \\
\hline Primer forward (L154N) & 5'-tgcttgtcagtgctcttgtattccgagcattcttccccag-3' \\
\hline Primer Reverse (L154N) & 5'-ctgggggaagaatgctcggaatacaagagcactgacaagca-3' \\
\hline & \\
\hline Template & $\Delta 2$-10F714SF717S / $\Delta$ PASCapF714SF717S \\
\hline Primer forward (F714S) & $\Delta 2$-10 / 'PASCap psGEM \\
\hline Primer Reverse (F714S) & 5'-ctgtccggcgcctcagccagagattccgac-3' \\
\hline Primer forward (F717S) & 5'-ctgctgtcggcttctctggctgaggcgccgg-3' \\
\hline Primer Reverse (F717S) & 5'-ccggcgcctcagccagagaagccgacagcag-3' \\
\hline
\end{tabular}




\subsubsection{Cloning into a vector for expression in mammalian cells}

$\triangle$ PASCap and $\Delta 2-10$ were cloned into pcDNA3 to be expressed in HEK-293 (Human Embryonic Kidney) cells. A BamH1-XhoI fragment from psGEM $\Delta 2-10$ containing the complete open reading frame was cloned into the corresponding sites of pcDNA3 (Thermo Scientific). pSGEM $\Delta 2-10$ was digested using BamHI-HF, XhoI and PvuI (New England Biolabs) to render a clearly distinguishable 3Kbp band, and pcDNA3 with BamH1-HF and Xhol. Insert and vector were run in a low melting point agarose gel, and the band was cut and purified using NucleoSpin Gel and PCR Clean-up (MACHEREY-NAGEL). Vector and fragment were ligated using T4 ligase (New England Biolabs). Vector and insert were mixed with a ratio of $1: 3$, then $1 \mu \mathrm{l}$ of T4 ligase was added and a final volume of $10 \mu \mathrm{l}$ was reached with autoclaved water. The reaction was incubated at $16^{\circ} \mathrm{C}$ overnight.

pcDNA3 $\Delta 2-10$ was then used as a template for cloning pcDNA3 $\Delta$ PASCap using in-Fusion cloning (see above Table 1).

\subsubsection{Cloning 5xmyc-tagged calmodulin}

Calmodulin was cloned into a pSGEM construct that had five repetitions in tandem of the myc tag (Lorinczi et al., 2015). Calmodulin (Addgene plasmid 47598 pKK233-hCaM)(Rhyner et al., 1992) fragment was obtained using restriction enzymes. Both constructs were digested NcoI and HindIII (New England Biolabs). Fragments were separated using gel-electrophoresis (low melting point agarose gel). The band was cut from the gel and purified with NucleoSpin Gel and PCR Clean-up (MACHEREYNAGEL). The vector and insert were ligated using T4 ligase (New England Biolabs). Vector and insert were mixed with a ratio of $1: 3$, then $1 \mu \mathrm{l}$ of $\mathrm{T} 4$ ligase was added and a final volume of $10 \mu \mathrm{l}$ was reached with autoclaved water. The reaction was incubated at $16^{\circ} \mathrm{C}$ overnight. 


\subsubsection{DNA isolation (Transformation)}

The ligation product was transformed into E. coli DH5 $\alpha$ cells. The DNA was added to the chemically competent cells and incubated for $30 \mathrm{~min}$ on ice. Then, a heat shock was performed for $1 \mathrm{~min}\left(42^{\circ} \mathrm{C}\right)$, followed by $3 \mathrm{~min}$ on ice. LB medium $(5 \mathrm{~g} / \mathrm{l}$ yeast extract, $10 \mathrm{~g} / \mathrm{l}$ Tryptone, $0,5 \mathrm{~g} / \mathrm{l} \mathrm{NaCl}, 1 \mathrm{~g} / \mathrm{l}$ glucose) was added and transformed bacteria were incubated for $1 \mathrm{~h}$ at $37{ }^{\circ} \mathrm{C}$, centrifuged and plated ot on 1.5\% LB-agar plates with the appropriate antibiotic selection $(100 \mu \mathrm{g} / \mu \mathrm{l}$ Ampicillin for pSGEM and pCDNA, $25 \mu \mathrm{g} / \mu \mathrm{l}$ Kanamycin for pLECI71). A mini-prep was then performed to isolate the product using NucleoSpin Plasmid (MACHEREY-NAGEL) . For larger DNA amounts. a midi-prep was performed using NucleoBond Xtra Midi (MACHEREY-NAGEL). The construct was sequenced for validation using the primers listed in Table 3. The correctness of the sequences was checked using Seqman Pro (version 15; DNASTAR)

Table 3. Primers for sequencing

\begin{tabular}{|l|l|}
\hline Primers for Sequencing & agctggctccaagcgtgcaa \\
\hline & aagatggctgtatagaaggt \\
\hline & ttcctgaagctctaccaggt \\
\hline & tgcaccgtctggaactccat \\
\hline & tggatgacctagatgtggagaa \\
\hline
\end{tabular}

\subsection{6 cRNA synthesis}

The constructs were linearized for RNA synthesis using NheI, which cuts at a single site after the Xenopus-optimized 3' untranslated region of pSGEM in all the constructs. Kv10.1 pSGEM, its respective mutations, and deletions were digested with NheI restriction enzyme. Mutants in pLeics71 were also digested with NheI. The product was purified using NucleoSpin Gel and PCR Clean-up (MACHEREY-NAGEL). Invitro transcription was then performed using mMESSAGE mMACHINE ${ }^{\text {TMT7 }}$ Transcription kit (Invitrogen Ambion) following the manufacturer's guidelines. 


\subsection{Oocyte preparation and injection}

Oocytes were surgically obtained from Xenopus laevis frogs as described in (Stühmer and Parekh, 1995). The isolated ovary follicle fragments were treated with collagenase (Nordmark) $1.4 \mathrm{mg} / \mathrm{ml}$ in $\mathrm{Ca}^{2+}$-free Barth buffer (Table 4 ) for $1.5 \mathrm{~h}$ at $18{ }^{\circ} \mathrm{C}$. The collagenase digests the connective tissue and aids in removing the follicular layer. Healthy immature oocytes at stage V-VI were selected and the follicular layer was mechanically removed with the aid of fine surgical forceps under a stereomicroscope. The oocytes were maintained in ND96 buffer (Table 4) with Tetracycline (USB; $50 \mu \mathrm{g} / \mathrm{ml}$ ), Amikacin (Enzo; $100 \mu \mathrm{g} / \mathrm{ml}$ ) and Ciprofloxacin (Enzo; $100 \mu \mathrm{g} / \mathrm{ml}$ ) at $18{ }^{\circ} \mathrm{C}$ (O'Connell et al., 2011). $0.5 \mathrm{mM}$ Theophylline (Sigma) was added to the ND96 buffer to inhibit oocyte maturation. It is essential to use immature oocytes as $\mathrm{K}_{\mathrm{v}} 10.1$ biophysical properties change during the cell-cycle (mature oocytes) (Bruggemann et al., 1997).

Oocytes were injected with cRNA within $36 \mathrm{~h}$ after isolation using a Nanoinjector (Drummond). The amount of injected cRNA ranged from 0.075 $0.5 \mathrm{ng} /$ oocyte (WT and point mutation), 0.075-5ng/oocyte (deletion mutants), and 810ng/ul (split channels). Injection pipettes were pulled from glass capillaries (3.5" Drummond \# 3-000-203-G/X DRUMMOND Scientific Company) and the tip was broken to the adequate size and shape under visual control. The injection pipette was first partly filled with mineral oil (Sigma) by suction from a drop deposited on a piece of paraffin paper, and then with cRNA by the same means. The oil is incompressible and ensures that the pressure is transferred to the cRNA regardless of the volume of cRNA in the pipette, decreasing the variability in the injected volume. Recordings were performed 1-5 days after injection. WT required least time to be expressed, some deletion mutants and split channels required longer time. 
Table 4.Buffers for oocytes isolation and maintenance

\begin{tabular}{|l|l|l|}
\hline Barth Buffer & $\mathrm{Ca}^{2+}$ free Barth Buffer & ND96 \\
\hline $88 \mathrm{mM} \mathrm{NaCl}$ & $88 \mathrm{mM} \mathrm{NaCl}$ & $96 \mathrm{mM} \mathrm{NaCl}$ \\
\hline $1 \mathrm{mM} \mathrm{KCl}$ & $1 \mathrm{mM} \mathrm{KCl}$ & $2 \mathrm{mM} \mathrm{KCl}$ \\
\hline $2.4 \mathrm{mM} \mathrm{NaHCO}_{3}$ & $2.4 \mathrm{mM} \mathrm{NaHCO}_{3}$ & $1.8 \mathrm{mM} \mathrm{CaCl}_{2}$ \\
\hline $0.82 \mathrm{mM} \mathrm{MgSO}_{4}$ & $0.82 \mathrm{mM} \mathrm{MgSO}_{4}$ & $1 \mathrm{mM} \mathrm{MgCl}_{2}$ \\
\hline $\left.0.33 \mathrm{mM} \mathrm{Ca} \mathrm{mO}_{3}\right)_{2}$ & $7.5 \mathrm{mM} \mathrm{Tris} \mathrm{Hcl}$ & $5 \mathrm{mM} \mathrm{HEPES}$ \\
\hline $0.41 \mathrm{mM} \mathrm{CaCl}_{2}$ & & $2.5 \mathrm{mM} \mathrm{Na}-$ pyruvate \\
\hline $7.5 \mathrm{mM} \mathrm{Tris} \mathrm{HCl}$ & & $0.5 \mathrm{mM} \mathrm{Theophylline}$ \\
\hline & & \\
\hline $\mathrm{pH}=7.4$ & $\mathrm{pH}=7.4$ & $\mathrm{pH}=7.55$ \\
\hline & & \\
\hline
\end{tabular}

\subsection{Cell culture and transfection}

HEK293 cells were maintained at $37{ }^{\circ} \mathrm{C}, 5 \% \mathrm{CO}_{2}$ using DMEM medium (Dulbecco's Modified Eagle Medium (1x) containing Pyruvate, 4.5g/l D-Glucose, and LGlutamine, Gibco (life technologies)) supplemented with 10\% FBS (Heat inactivated Fetal Bovine Serum, Gibco (life technologies)). Cells were detached using 1ml TrypsinEDTA (Gibco) for $5 \mathrm{~min}$. Then, $9 \mathrm{ml}$ medium was added to inhibit Trypsin.

The cells were seeded at a concentration of 100,000/well onto fibronectincoated glass coverslips in 6-well plates. They were co-transfected 12-24h after seeding with a plasmid for GFP expression (Lonza) and the respective mutant ( $\Delta 2-10$.pcDNA) or $(\triangle \mathrm{PASCap} . \mathrm{pcDNA})$. Transfection was performed using Lipofectamine 3000

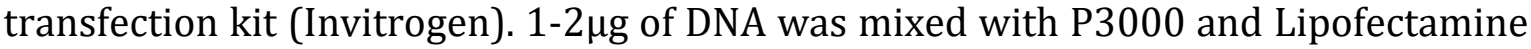
3000 reagents in Opti-MEM medium (Invitrogen), incubated for 10 minutes to form liposomes and then added to the wells.

Stable transfected HEK-293 expressing human $\mathrm{K}_{\mathrm{v}} 10.1$ in pTracer-CMV were maintained in DMEM/nutrient mixture F-12 with Glutamax-I (GIBCO BRL), 10\% FCS and $300 \mu \mathrm{g} / \mathrm{ml}$ Zeocin. 


\subsection{Recordings}

\subsubsection{Two-electrode voltage clamp (TEVC)}

Recordings of currents through channels expressed in Xenopus laevis oocytes were performed at room temperature using two-electrode voltage-clamp (TEVC). Two electrodes (potential and current electrode) were inserted into the oocyte to perform voltage-clamp measurements. Pipettes were pulled using temperature-controlled pipette puller PIP 5 (HEKA Elektronik). The pipettes were filled with $2 \mathrm{M} \mathrm{KCl} \mathrm{(3M} \mathrm{for}$ some experiments) and had a resistance of $0.4-1.2 \mathrm{M} \Omega$. Recordings were performed in Normal Frog Ringer solution (NFR, Table 5) as external solution. External $\mathrm{K}^{+}$ concentration was raised to $60 \mathrm{mM}$ to shift the equilibrium potential for $\mathrm{K}^{+}$and obtain tail currents at repolarizing voltages. $\mathrm{Cl}^{-}$free $\mathrm{NFR}$ was used to avoid $\mathrm{Cl}^{-}$contamination of outward current when increasing $\mathrm{Ca}^{2+}$ concentration or applying hyperpolarizing pre-pulses. In this case, agar bridges replaced the reference electrodes $(2 \%$ agar in $2 \mathrm{M}$ $\mathrm{NaCl}$ ). When indicated, $1 \mathrm{mM}$ or $5 \mathrm{mM} \mathrm{MgCl}_{2}$ (MERCK) was added to the respective solution to slow down the movement of S4 (Terlau et al., 1996). In order to raise the intracellular $\mathrm{Ca}^{2+}$ concentration, $5 \mu \mathrm{M}$ Ionomycin (Abcam) and $5 \mu \mathrm{M}$ Thapsigargin (Abcam) were applied in $\mathrm{Cl}^{-}$-free NFR. Ionomycin is a ionophore that allows $\mathrm{Ca}^{2+}$ entry into the oocytes (Yoshida and Plant, 1992), while thapsigargin is an inhibitor of the endoplasmic reticulum $\mathrm{Ca}^{2+}$-ATPase, preventing $\mathrm{Ca}^{2+}$ reuptake into the endoplasmic reticulum and thereby emptying the $\mathrm{Ca}^{2+}$ stores (Sehgal et al., 2017; Thurman et al., 2000). A saturating concentration of both ensures a relatively sustained increase in $\mathrm{Ca}^{2+}$ level (Lorinczi et al., 2016). In disulphide bond formation experiments (mutant R8C), the oxidizing reagent $\mathrm{TbHO}_{2}$ (Tert-butyl hydroperoxide) was perfused for $3 \mathrm{~min}$, followed by the reducing reagent DTT (1,4 Dithio-threitol) (de la Pena et al., 2015). $2 \mathrm{mM} \mathrm{TbHO}_{2}$ (Sigma-Aldrich) and $5 \mathrm{mM}$ DTT (Fluka) were dissolved in NFR immediately before recordings.

Patchmaster software (HEKA Elektronik) was used to design the voltage protocols and record the respective current responses. Leak subtraction protocol $(\mathrm{P} / \mathrm{n})$ was used when indicated. A holding potential of $-100 \mathrm{mV}$ was used throughout 
the experiments. Digital to analogue conversion was performed using the ITC16interface of an EPC9 patch-clamp amplifier (HEKA Elektronik). The data was acquired using a TurboTEC 10-CD amplifier (NPI Electronics), filtered at $1.3 \mathrm{KHz}$ and sampled at $10 \mathrm{KHz}$. The data was analyzed using Fitmaster (version 2. and above, HEKA Elektronik) and IgorPro (version 6, WaveMetrics).

Table 5. Buffers for TEVC recordings

\begin{tabular}{|l|l|l|}
\hline $\begin{array}{l}\text { Normal Frog Ringer } \\
\text { solution (NFR) }\end{array}$ & $\begin{array}{l}60 \mathrm{mM} \mathrm{K} \quad \text { external } \\
\text { solution }\end{array}$ & $\mathrm{Cl}^{+}$free NFR \\
\hline $115 \mathrm{mM} \mathrm{NaCl}$ & $57.5 \mathrm{mM} \mathrm{NaCl}$ & $\begin{array}{l}115 \quad \mathrm{mM} \text { Na- } \\
\text { methanesulfonate }\end{array}$ \\
\hline $2.5 \mathrm{mM} \mathrm{KCl}$ & $60 \mathrm{mM} \mathrm{KCl}$ & $2.5 \mathrm{mM} \mathrm{KOH}$ \\
\hline $10 \mathrm{mM} \mathrm{HEPES}$ & $10 \mathrm{mM} \mathrm{HEPES}$ & $10 \mathrm{mM} \mathrm{HEPES}$ \\
\hline $1.8 \mathrm{mM} \mathrm{CaCl} 2$ & $1.8 \mathrm{mM} \mathrm{CaCl}_{2}$ & $1.8 \mathrm{mM} \mathrm{Ca}(\mathrm{OH})_{2}$ \\
\hline $\begin{array}{l}\mathrm{pH}=7.2 \\
\text { adjusted with }(\mathrm{NaOH})\end{array}$ & $\begin{array}{l}\mathrm{pH}=7.4 \\
\text { adjusted with }(\mathrm{KOH})\end{array}$ & $\begin{array}{l}\mathrm{pH}=7.2 \\
\text { adjusted with } \\
\text { (Methanesulfonic acid/ } \\
\text { NaOH) }\end{array}$ \\
\hline
\end{tabular}

\subsubsection{Patch-Clamp}

Recordings were performed 24-48 h after transfection (or after plating of stably transfected cells). Transfected HEK cells, identified through GFP fluorescence, were patched at room temperature. The standard external solution (Table 6) was used to record current-voltage (IV) plots, while $60 \mathrm{mM} \mathrm{K}^{+}$solution (Table 6) was used for square pulse protocol. The pipette solution is also listed in Table 6.

Pipettes were pulled using a temperature-controlled pipette puller PIP 5 (HEKA Elektronik) from \#1 glass capillaries (WPI). Pipettes had resistances of 2-4 M $\Omega$ when filled with the standard intracellular solution. The data was acquired and digitized using an EPC10 plus amplifier (HEKA Elektronik). The data was sampled at 20 or 50 $\mathrm{KHz}$ and filtered at 4 or $10 \mathrm{KHz}$, respectively. The digitized data was recorded via Patchmaster software, which was also used to design the applied voltage protocols. Leak subtraction protocol $(\mathrm{P} / \mathrm{n})$ was applied. Analysis of the data was performed using Fitmaster (HEKA Elektronik) and Igor Pro (Wavemetrics). 
Table 6. Buffers for Patch-Clamp recordings

\begin{tabular}{|c|c|c|}
\hline Internal solution & External Solution & $\begin{array}{lll}60 \mathrm{mM} & \mathrm{K} & \text { External } \\
\text { solution } & & \end{array}$ \\
\hline $100 \mathrm{mM} \mathrm{KCl}$ & $160 \mathrm{mM} \mathrm{NaCl}$ & $100 \mathrm{mM} \mathrm{NaCl}$ \\
\hline $\begin{array}{l}45 \mathrm{mM} \quad \mathrm{N} \text {-methyl D- } \\
\text { glucamine } \\
\text { (SIGMA) }\end{array}$ & $2.5 \mathrm{mM} \mathrm{KCl}$ & $62.5 \mathrm{mM} \mathrm{KCl}$ \\
\hline 10mM HEPES & $2 \mathrm{mM} \mathrm{CaCl}_{2}$ & $2 \mathrm{mM} \mathrm{CaCl}_{2}$ \\
\hline 10mM BAPTA & $1 \mathrm{mM} \mathrm{MgCl} 2$ & $1 \mathrm{mM} \mathrm{MgCl} 2$ \\
\hline & 8mM Glucose & $\begin{array}{l}\text { 8mM Glucose } \\
\text { Fluka }\end{array}$ \\
\hline & 10mM HEPES & 10mM HEPES \\
\hline
\end{tabular}

\subsubsection{Analysis}

The equilibrium potential was calculated from fitting IV curves recorded in 60 $\mathrm{mM} \mathrm{K}^{+}$solution. Then, conductance (G) was deduced from end-pulse current (I), determined as average current in the final $\mathrm{XX} \%$ of the depolarizing pulse using the following equation:

$$
G=\frac{I}{V m-V e q}
$$

\section{Equation 1}

The difference between membrane potential $\left(V_{m}\right)$ and $\left(V_{\text {eq }}\right)$ is the driving force. Once $V_{m}$ reaches $V_{\text {eq }}$, the driving force is 0 , no current is detected. As long as $V_{m}<V_{\text {eq }}$ an inward current can be detected, while $V_{m}>V_{\text {eq }}$ induces outward current. We used two external solutions with different $\mathrm{K}^{+}$concentration: $60 \mathrm{mM} \mathrm{K}^{+}$solution $\left(\mathrm{V}_{\text {eq }} \sim\right.$ $20 \mathrm{mV}$ ) and NFR $\left(2.5 \mathrm{mM} \mathrm{K}^{+}, \mathrm{V}_{\text {eq }} \sim-100 \mathrm{mV}\right) .60 \mathrm{mM} \mathrm{K}^{+}$solution was used for GV protocols to have inward currents at negative potentials $(<-20 \mathrm{mV})$. The inward tail current obtained during the repolarizing pulse reflects the deactivation kinetics of the channels. The amplitude of the tail at time $=0$ is proportional to the conductance (G) at the previous depolarizing pulse. Tails were fitted with a double exponential function:

$$
f(t)=A 0+A 1 e^{-\frac{t}{\tau 1}}+\mathrm{A} 2 e^{-\frac{t}{\tau 2}}
$$

\section{Equation 2}




$$
\mathrm{Y}=\mathrm{A} 0+\mathrm{A} 1+\mathrm{A} 2
$$

\section{Equation 3}

Conductance-voltage (GV) plots usually follow a sigmoidal response. Conductance was normalized to the maximum (G/Gmax) and a Boltzmann equation (sigmoidal curve) was used for fitting.

$$
f(V)=\frac{1}{1+e^{-\left(V_{m}-V_{h}\right) / K}}
$$

\section{Equation 4}

$\mathrm{V}_{\mathrm{m}}$ is the membrane potential, $\mathrm{V}_{\mathrm{h}}$ is the potential at which half the maximum response is reached, and $K$ represents the slope $(K=R T / z F)$, where $R$ is the gas constant, $\mathrm{T}$ is the temperature in Kelvin, $\mathrm{z}$ is the charge, and $\mathrm{F}$ is the Faraday constant. The mutants showed a biphasic GV, which we fitted with a two-sigmoidal equation and a weight which represents the transition between the two components.

$$
\begin{gathered}
f(V)=\mathrm{A} 0+\left(\frac{\mathrm{A} 1}{1+\mathrm{e}^{\frac{\mathrm{Vh} 1-\mathrm{V}}{\mathrm{K} 1}}}\right) *\left(1-\left(\frac{1}{1+\mathrm{e}^{\frac{\mathrm{Vh} 3-\mathrm{V}}{\mathrm{K} 3}}}\right)\right)+ \\
\left(\frac{\mathrm{A} 2}{1+\mathrm{e}^{\frac{\mathrm{Vh} 2-\mathrm{V}}{\mathrm{K} 2}}}\right) *\left(\frac{1}{1+\mathrm{e}^{\frac{\mathrm{Vh} 3-\mathrm{V}}{\mathrm{K} 3}}}\right)
\end{gathered}
$$

\section{Equation 5}

To evaluate activation kinetics, time to reach $80 \%$ of maximum current was used (Risetime80\%). 


\subsection{Pull-down assay}

\subsubsection{Buffer preparation}

Buffers were prepared with different $\mathrm{Ca}^{2+}$ concentrations $(0,100 \mathrm{nM}, 1 \mu \mathrm{M})$. EGTA was used to buffer $\mathrm{Ca}^{2+}$ to the desired concentration. $\mathrm{Ca}^{2+}$ replaces protons when chelated by EGTA. The release of protons results in decreased $\mathrm{pH}$, which was adjusted to counteract the acidity.

Table 7. Buffers for immunoprecipitation

\begin{tabular}{|c|c|c|}
\hline Lysis Buffer & Wash buffer & TBS buffer \\
\hline $1 \%$ Triton $X-100$ & $0.1 \%$ Triton $\mathrm{X}-100$ & $150 \mathrm{mM} \mathrm{NaCl}$ \\
\hline $150 \mathrm{mM} \mathrm{NaCl}$ & $300 \mathrm{mM} \mathrm{NaCl}$ & 50mM Tris-HCl \\
\hline 50 mM HEPES pH 7.4 & 50 mM HEPES pH 7.4 & pH 7.6 \\
\hline $\begin{array}{l}\text { Complete preotease } \\
\text { inhibitors, EDTA free } \\
\text { (Roche) }\end{array}$ & $\begin{array}{l}\text { Complete preotease } \\
\text { inhibitors, EDTA free } \\
\text { (Roche) }\end{array}$ & \\
\hline $5 \mathrm{mM}$ EGTA & $5 \mathrm{mM}$ EGTA & \\
\hline $\mathrm{CaCl}_{2}(0,2 \mathrm{mM}, 2.6 \mathrm{mM})$ & $\mathrm{CaCl}_{2}(0,2 \mathrm{mM}, 2.6 \mathrm{mM})$ & \\
\hline
\end{tabular}

\subsubsection{Immunoprecipitation (IP)}

Pull-down assay was designed to assess the binding of calmodulin to $\mathrm{K}_{\mathrm{v}} 10.1$ at $\mathrm{Ca}^{2+}$ level equivalent to basal $\mathrm{Ca}^{2+}(100 \mathrm{nM})$ (Cork et al., 1987). cRNA of 5xmyccalmodulin (10ng) and $\mathrm{K}_{\mathrm{v}} 10.1$ (0.5ng) were co-injected into Xenopus laevis oocytes. Oocytes were lysed at different $\mathrm{Ca}^{2+}$ concentrations $(0 \mathrm{nM}, 100 \mathrm{nM}, 1 \mu \mathrm{M})$, using $20 \mu \mathrm{l} /$ oocytes of lysis buffer (Table7), homogenized by pipetting repeatedly and then incubated on ice for 30min. As a control, non-injected oocytes and oocytes injected with Kv10.1 or myc-tagged alone were used. 20-30 oocytes were used for each group. The three control groups were lysed at $0 \mathrm{nM} \mathrm{Ca}^{2}$. The lysate was centrifuged at $4{ }^{\circ} \mathrm{C}$ for $3 \mathrm{~min}$ at $16000 \mathrm{xg}$ and the pellet was discarded. $10 \%$ of the supernatant was set aside to be loaded on the gel as a control. The supernatant was used for immunoprecipitating 5xmyc tagged calmodulin. All incubation steps were performed at $4{ }^{\circ} \mathrm{C}$ and samples were gently mixed using a rocking wheel for $1.5 \mathrm{~h}$. First, samples were incubated with 
$15 \mu$ Protein G magnetic beads (New England Biolabs) as a clearing step to eliminate non-specific binding to the beads. A magnetic stand (New England Biolabs) was used in all steps to collect the beads. The pre-clearing beads were then discarded, and the samples were incubated with $3 \mu \mathrm{g}$ of anti-myc antibody (Sigma) for 1.5h. Finally, samples were incubated with $30 \mu \mathrm{l}$ Protein $\mathrm{G}$ beads to bind the antibody-target complex that was formed. The target includes the myc-tagged calmodulin and proteins that bind to calmodulin. Beads were then washed three times using the buffer in Table 7. The respective $\mathrm{Ca}^{2+}$ concentration was maintained throughout the procedure. Finally, proteins were recovered by addition of $25 \mu$ l NuPAGE LDS sample buffer (Thermo Scientific) and heating at 70 degree for $10 \mathrm{~min}$.

\subsubsection{Western blot}

A precast gel (NuPAGE 4 to 12\%, Bis-Tris) was assembled in a X-Cell SureLock electrophoresis cell (Invitrogen). The electrophoresis chamber, close to the gel, was filled with $200 \mathrm{ml}$ of 1 XMOPS running buffer (Table 8) and $500 \mu \mathrm{l}$ antioxidant (to avoid recovery of disulphide bonds during the electrophoretic separation; NuPAGE Invitrogen). The rest of the electrophoresis cell was filled with $300 \mathrm{ml}$ 1xMOPS running buffer. $5 \mu \mathrm{l}$ molecular weight markers (Broad range protein marker, New England Biolabs) and the IP samples $(25 \mu \mathrm{l})$ were loaded on the gel. The separation was performed at $200 \mathrm{~V}$ for $50 \mathrm{~min}$. After separation, the proteins were transferred from the gel to a nitrocellulose membrane (Invitrogen). The gel, the nitrocellulose membrane, four pieces of Whatman paper and two sponge layers were soaked in transfer buffer (Table 8). Then, the sponge and the Whatman paper were used to "sandwich" the gel and the membrane in a gel holder cassette (Bio-Rad). The transfer sandwich was assembled in a transfer tank Mini Trans-Blot Cell (BioRad) so that the gel faced the cathode while the membrane faced the anode. The chamber was filled with transfer buffer (Table 8) and a magnetic stirrer was used to stir the solution during transfer. Negatively charged protein samples were then transferred using voltage from the gel to the membrane. The transfer was first performed with a gradient increase in voltage from 10 to $40 \mathrm{~V}$ every $10 \mathrm{~min}$ in $10 \mathrm{~V}$ steps. Finally, 50V was applied for $40 \mathrm{~min}$. 
The nitrocellulose membrane was then probed with anti-myc antibody (Sigma). To minimize non-specific binding the blot was incubated with 5\% albumin (Sigma) (dissolved in TBS-T) for $1 \mathrm{~h}$ on a shaker. The blot was then incubated with anti-myc (1:1000, diluted with TBS-T) at $4{ }^{\circ} \mathrm{C}$ overnight on a shaker. The blot was washed 7 times with a large volume of $\mathrm{H}_{2} \mathrm{O}$, followed by 5 min incubation with TBS-T. TBS-T was discarded and peroxidase (HRP)-conjugated anti-mouse antibody (Amersham, 1:7000, diluted with TBS-T) was then added to the blot and incubated for $2 \mathrm{~h}$ on a shaker at 4 ${ }^{\circ} \mathrm{C}$. The membrane was washed seven times with excess water and $5 \mathrm{~min}$ with TBS-T, and finally developed using Immobilon ECL chemiluminescence substrate (Millipore). HRP acts as a catalyst for the oxidation of the chemiluminescent substrate with hydrogen peroxide, emitting light. The light was then detected using a Chemostar (Intas) gel documentation system. To probe with anti- $K_{v} 10.1$, the blot was first stripped using Restore stripping buffer (Invitrogen), and the same immunoblotting protocol was then followed. The blot was blocked with 5\% albumin, then incubated with anti-Kv10.1 (polyclonal anti-Kv10.a1, 1:1500) overnight. After washing, the blot was incubated with anti-rabbit antibody. The result presented corresponds to one trial $(\mathrm{N}=1)$.

Table 8. Buffers for western blot

\begin{tabular}{|l|l|l|}
\hline Transfer Buffer & $\begin{array}{l}\text { NuPAGE } \\
\text { Running Buffer (20X) }\end{array}$ & TBS-T Buffer \\
\hline $10 \mathrm{mM} \mathrm{NaHCO}_{3}$ & $50 \mathrm{mM}$ MOPS & $20 \mathrm{mM}$ Tris-HCl \\
\hline $3 \mathrm{mM} \mathrm{Na}_{2} \mathrm{CO}_{3}$ & $50 \mathrm{mM}$ Tris Base & $150 \mathrm{mM} \mathrm{NaCl}^{\text {Tween }}{ }^{\circledR} 20 \quad$ detergent \\
$0.01 \% \mathrm{SDS}$ & $0.1 \%$ SDS & \\
\hline $20 \%$ methanol & $1 \mathrm{mM}$ EDTA $)$ \\
\hline & & \\
\hline $\mathrm{pH}>9$ & pH 7.7 & \\
\hline
\end{tabular}




\section{Results}

Our study aimed to explore an alternative model to the "mechanical lever" previously hypothesized to govern the gating of all voltage-gated $\mathrm{K}^{+}$channels, including those in the $K C N H$ family. The peculiar intracellular domains that characterize this family have drawn our attention, and we hypothesized that the intracellular domains play a role in the gating of $\mathrm{K}_{\mathrm{v}} 10.1$. To test this hypothesis, we studied the behavior of a series of deletions in the intracellular domains: three $\mathrm{N}$ terminal deletions ( $\Delta 2-10, \Delta$ PASCap (2-25), $\Delta$ eag (2-135)) and a C-terminal deletion $\triangle$ CNBHD (525-697). We also studied a point mutation (E600R) that disrupts the interaction between intracellular domains (CNBHD and PASCap domain). $\Delta 2-10$, $\triangle$ PASCap, $\triangle$ eag, $\triangle$ CNBHD and E600R refer to deletions or mutations of the corresponding residues or domains of $\mathrm{K}_{\mathrm{v}} 10.1$ (see methods). The full-length channel "pSGEM.K $\mathrm{K}_{\mathrm{v}} 10.1$ " will be referred to as wild-type "WT". cRNA of the respective mutants was injected into Xenopus laevis oocytes to perform two-electrode voltage clamp (TEVC) recordings. We were immediately intrigued by the conspicuous biphasic behavior observed with the N-terminal deletions and reproduced by the E600R mutation. Therefore, our efforts were directed to explain the behavior of these mutants.

\subsection{The initial N-terminus mutant $\mathrm{R} 8 \mathrm{C}$ forms disulfide bridges with endogenous Cys residue(s) to stabilize a closed conformation}

At the beginning of our study, the Cryo-EM structure of rat $\mathrm{K}_{\mathrm{v}} 10.1$ (Whicher and MacKinnon, 2016) revealed that the initial N-terminus segment could lie in a cavity close to critical molecular players in gating (S4, S4-S5, C-linker, CNBHD). However, the first nine residues were not resolved in that structure, which might indicate considerable flexibility of the segment. To assess the proximity of the initial residues to the molecular candidates in the cavity, we inserted a Cys mutation at position 8 (R8C). We then tested the impact of the membrane-permeable oxidizing reagent 
terbutyl hydroperoxide $\left(\mathrm{TbHO}_{2}\right)$ on the properties of the current. $\mathrm{TbHO}_{2}$ induces the formation of disulfide bonds (S-S) when Cys residues are close enough to each other since the length of a Cys-Cys bridge is approximately $2.04 \AA$ (Chaney and Steinrauf, 1974; Wiedemann et al., 2020). If R8C is close enough to an endogenous Cys, the formation of an S-S bond will limit the displacement of the initial segment of the channel. In principle, the disulfide bridge could lock the channel in an open or a closed conformation. A reducing reagent (DTT) was then applied to revert the effect. The protocol was adopted from (de la Pena et al., 2015).

Wild-type (WT) and R8C cRNA were injected into Xenopus laevis oocytes and TEVC recordings were performed. A test pulse $(+40 \mathrm{mV})$ was repeated every 20 s during the perfusion of the reagents. Oocytes were held at $-100 \mathrm{mV}$ to allow the channel to close in between pulses. The perfusion of $2 \mathrm{mM} \mathrm{TbHO}_{2}$ for 3 min resulted in a mild current reduction of WT currents and a marked inhibition of R8C (Fig. 5). The current inhibition indicates stabilization of a closed conformation. The effect was recovered by 5 min perfusion with $5 \mathrm{mM}$ DTT. The recovery was almost complete in R8C, which further indicated the formation of disulfide bridges.

To identify the endogenous Cys interacting with R8C, we mutated three Cys residues (C214, C640, C575) that in the cryo-EM structure occupy positions that putatively could interact with the N-terminal end of the channel. C214 is located at the bottom of S1 and could interact with the N-terminus of the same subunit. C575 and C640 lie within the CNBHD and are very close to each other and in proximity to the Nterminus of the opposite subunit (Fig43. The double mutants R8C.C214A, R8C.C640A, R8C.C575A and the triple mutant R8C.C575A.C640A still showed inhibition of the same magnitude as the single point mutants (percent inhibition after perfusing $\mathrm{TbHO}_{2}$ for $3 \min : \mathrm{WT}=11 \pm 1 \%$; R8C = $32 \pm 1.7 \%$, R8C.C214A = $37 \pm 1.4 \%$, R8C.C640A $=27 \pm 1.1 \%$, R8C.C575A $=29 \pm 4.3 \%$, R8C.C575A.C640A.= $32 \pm 2 \%$; mean \pm SEM (standard error of the mean)), indicating that the partner of R8C is either a different endogenous residue or that both interactions (R8C-C214 or R8C-C575.C640) can occur, and one is sufficient to stabilize the closed conformation. A quadruple mutant will solve this question, but we did not pursue these mutants because the only structure available is in an active but closed conformation, while the relevant relations are expected to be those in the resting state. 
Although the partner of Cys 8 remains to be identified, these experiments indicate that the $\mathrm{N}$-terminal end moves during channel activation and fixing its position through a disulfide bond reduces the current. We decided to perform deletions in this region to determine their impact on channel gating. We aimed to test whether the interaction between the intracellular domains substituted the direct coupling through S4-S5 in Kv10.1. 


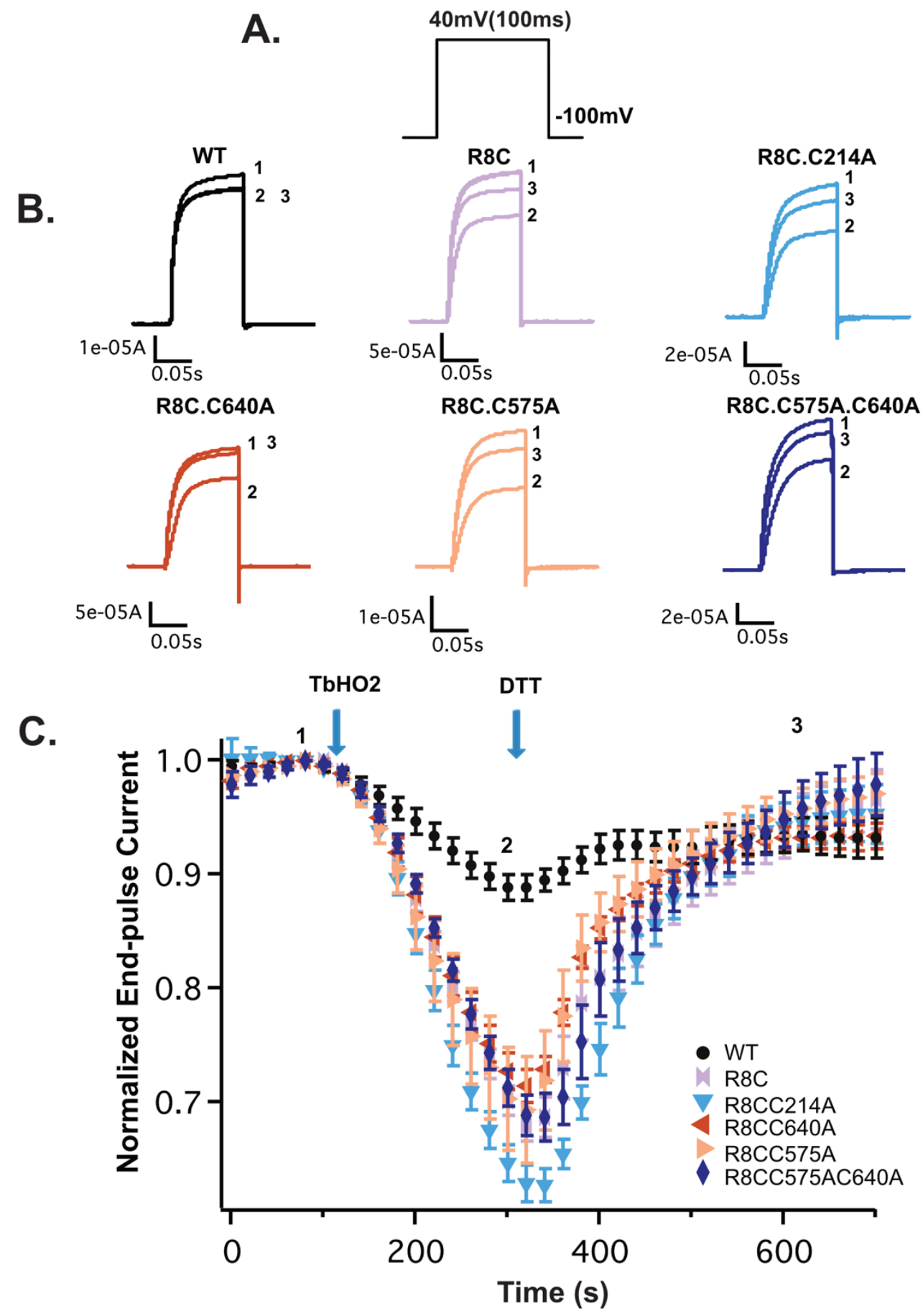

Figure 5. R8C reacts with an endogenous Cys to stabilize a closed conformation A) Test pulse repeated with $20 \mathrm{~s}$ interval B) Representative traces. 1. Control (NFR) 2. TbHO2 (3min) 3. DTT (5min) C) Normalized end-pulse current of repeated test pulse plotted against time. $2 \mathrm{mM}$ TbHO2 was perfused for $3 \mathrm{~min}$, followed by $5 \mathrm{mM}$ DTT for 5 min. Numbers refer to the representative traces shown in B. 


\subsection{N-terminal deletions and E600R unveil a biphasic behavior}

\subsubsection{Deletion of the first ten residues}

cRNA encoding a channel lacking the first ten N-terminal residues $(\Delta 2-10)$ was injected into Xenopus laevis oocytes. Oocytes were perfused with $60 \mathrm{mM} \mathrm{K} \mathrm{K}^{+}$external solution, held at $-100 \mathrm{mV}$, and voltage steps $(10 \mathrm{mV})$ from $-100 \mathrm{mV}$ to $+120 \mathrm{mV}$ with $30 \mathrm{~s}$ interval were applied in TEVC experiments. Conductance $(G)$ was calculated using the end-pulse current (Eq. 1); the mean amplitude during the last $20 \mathrm{~ms}$ of depolarization was divided by the driving force obtained subtracting the measured reversal potential from the test potential. The results are shown in (Fig. 6). For this mutant, the repolarizing potential chosen was $-160 \mathrm{mV}$ due to the slow deactivation at $-100 \mathrm{mV}$. Fig. 6C shows representative current traces using the protocol schematically depicted in Fig. 6A. The channel activated already at relatively hyperpolarized potentials as compared to WT. The current increased progressively with stronger depolarizations, but from $+10 \mathrm{mV}$ on, inward rectification was evident. Surprisingly, the rectification was overcome after $+60 \mathrm{mV}$, and the current progressively increased further (Fig. 6E). This observation also translated into the amplitude of the tail current, which increased until $0 \mathrm{mV}$ but decreased after that (Fig. 6D). However, in contrast to the outward current, the tail current amplitude remained constant after stimuli positive to $+60 \mathrm{mV}$. To visualize the difference between end-pulse and tail current, we compared the conductance calculated using Eq. 1 to that calculated using Eq. 2 and Eq. 3 (Fig. 7). Fitting the tail current with a double exponential can be used to deduce the current amplitude at $\mathrm{t}=0$ (Eq. 2 and Eq. 3), which is proportional to the overall conductance at the test potential. This discrepancy indicates that strong potentials drive the channel into a state that favors outward over inward current. 

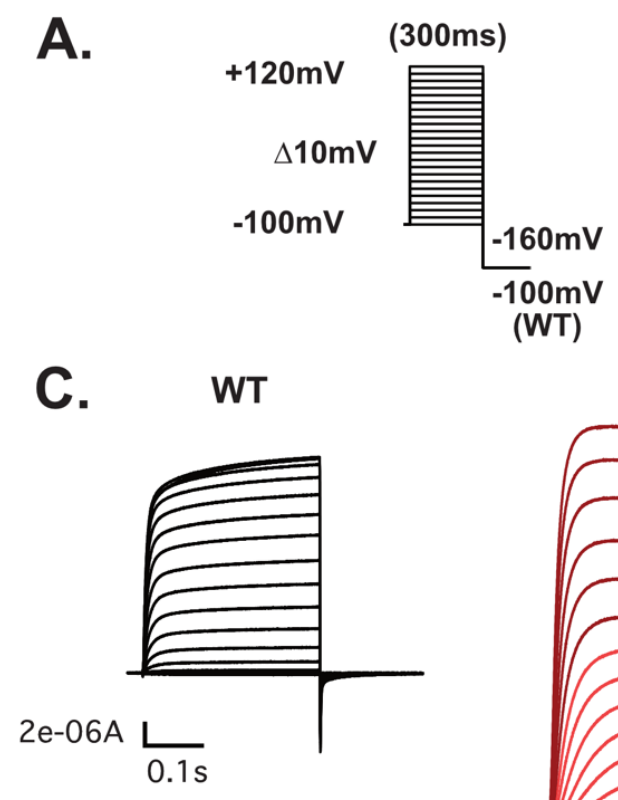

E.

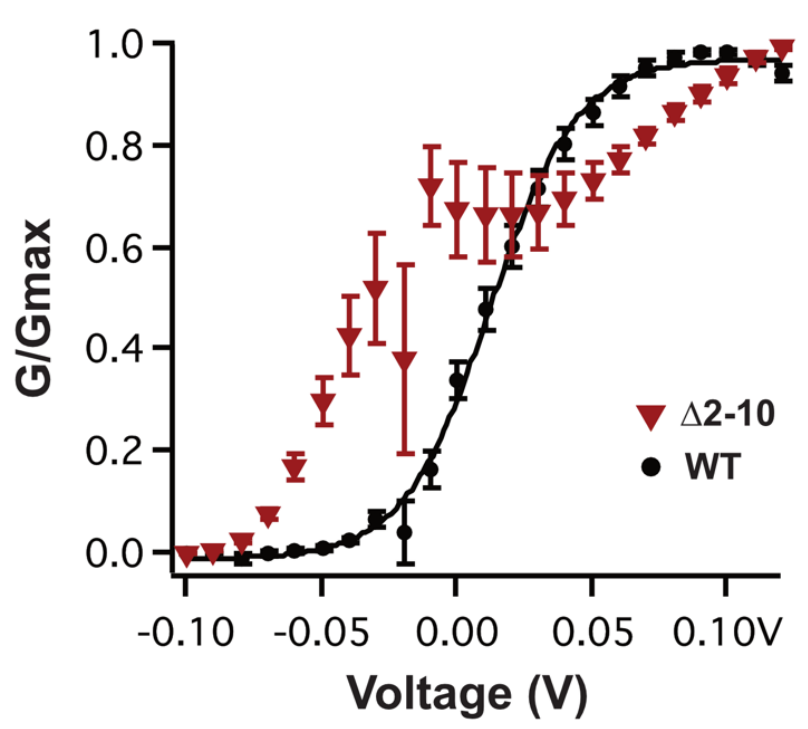

B.

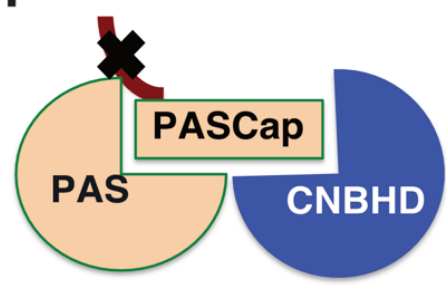

D.

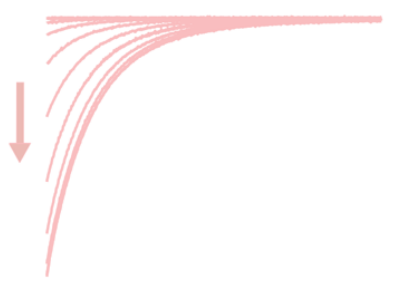

$-100 \mathrm{mV}$ to $0 \mathrm{mV}$

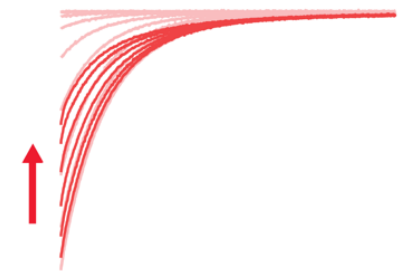

$+10 \mathrm{mV}$ to $+60 \mathrm{mV}$

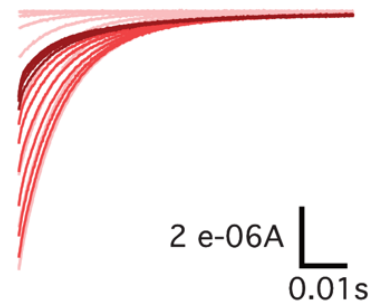

$+70 \mathrm{mV}$ to $+120 \mathrm{mV}$

Figure 6. $\mathrm{N}$-terminal deletion $(\Delta 2-10)$ unveils a biphasic behavior. A) Schematic representation of the voltage stimulus applied. A leak subtraction protocol $(\mathrm{P} / \mathrm{N})$ was used since we did not observe differences between its presence or absence. Recordings were performed in $60 \mathrm{mM} \mathrm{K}^{+}$solution. B) Cartoon representing $\Delta 2-10$. C) Representative current response. The color code is the same as shown in D. D) Tail current in C. E) Normalized conductance plotted vs. voltage (N: WT=11 $\Delta 2-10=6$; mean \pm SEM): GV. G was calculated using end-pulse current (Eq. 1). WT GV was fitted with a sigmoid: $\left(\mathrm{A}_{1}=0.98, \mathrm{Vh}_{1}=0.012 \mathrm{~V}, \mathrm{~K}_{1}=0.015, \mathrm{~N}=11 ; E q .4\right)$ 

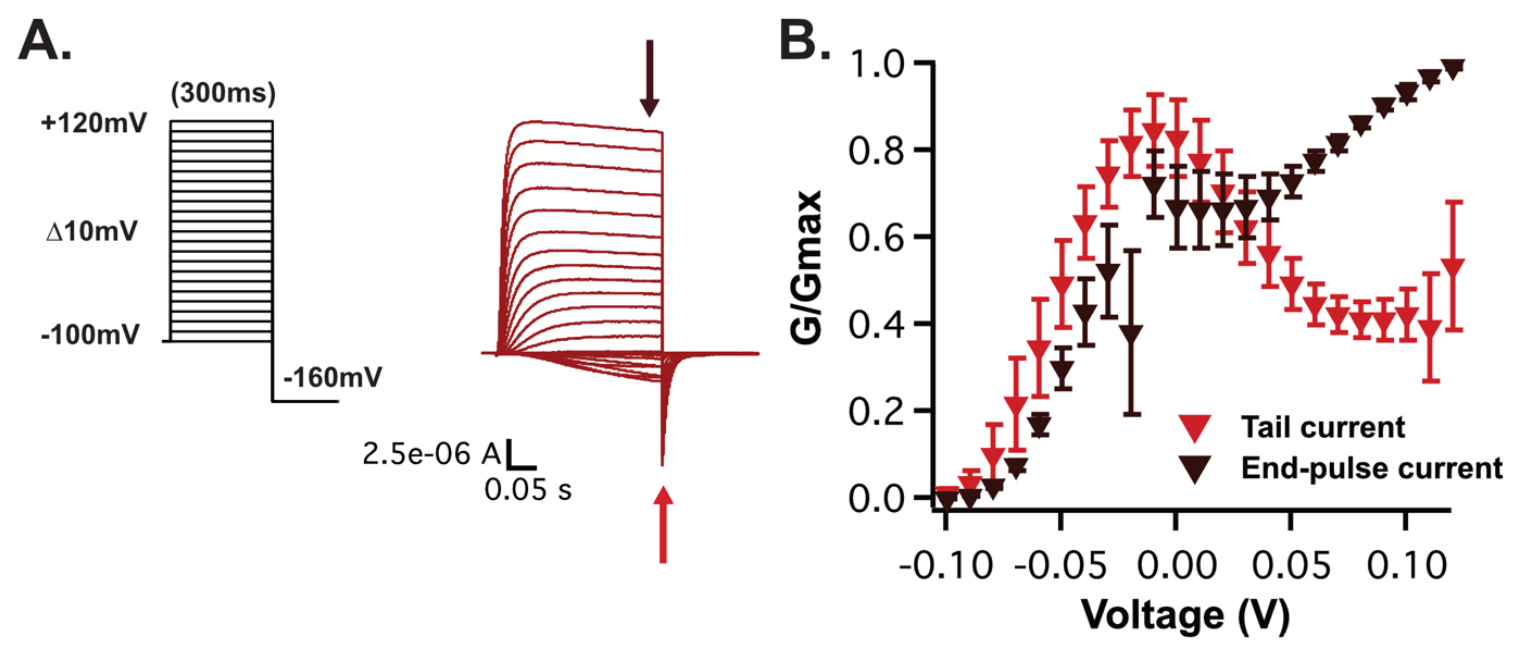

Figure 7. Tail current deviates from end-pulse current indicating outward rectification at strong potentials. A) Voltage protocol (left), Representative current traces (right). Arrows indicate end-pulse and tail current. B) Normalized conductance was plotted against voltage (GV) $(\mathrm{N}=6$; mean $\pm \mathrm{SEM})$. $\mathrm{G}$ was calculated using end-pulse current (Eq. 1), and tail current using Eq. 2 and Eq. 3.
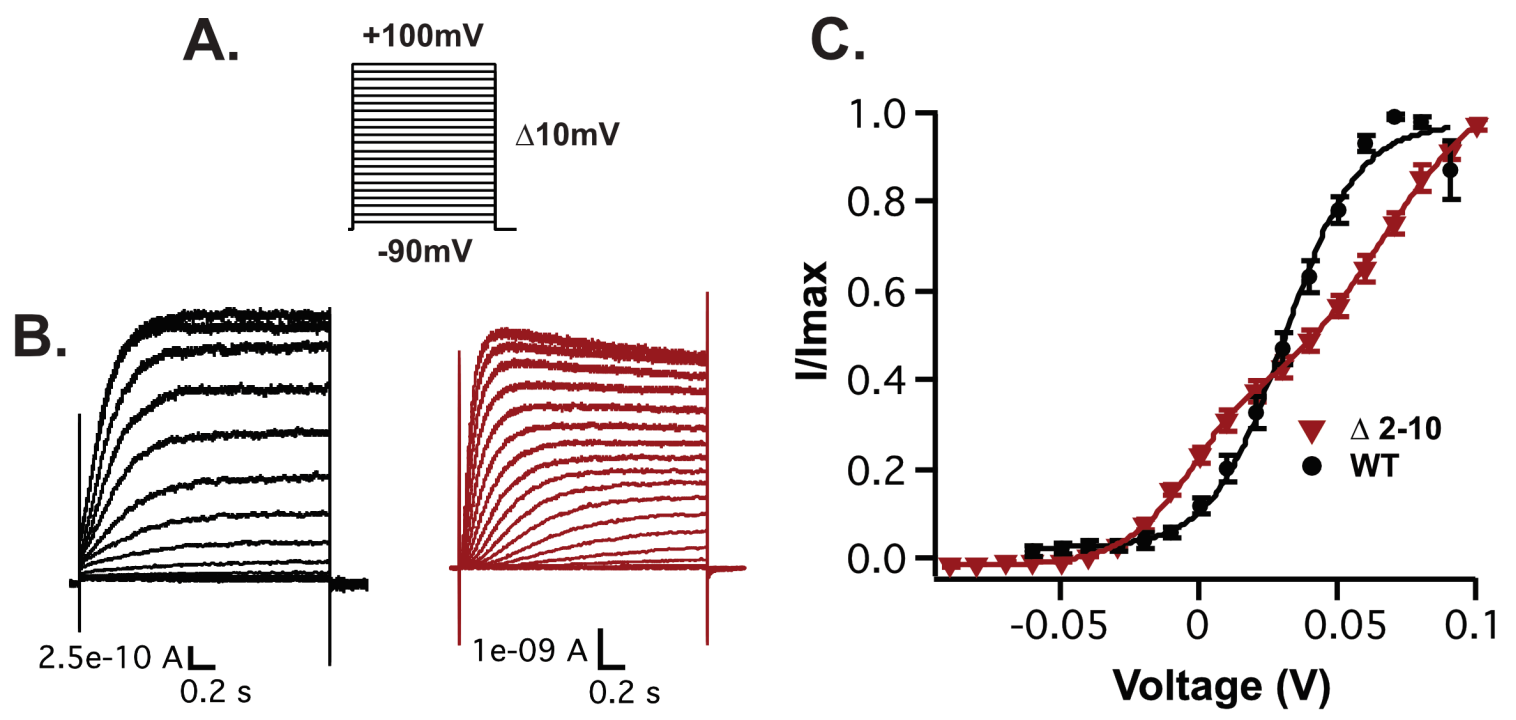

Figure 8. $\Delta 2$-10 shows similar biphasic behavior in HEK293 cells. A) Voltage protocol applied. B) Representative recordings of WT and $\Delta 2-10$ (2s) C) Normalized end-pulse current vs. voltage (mean \pm SEM). IV was fitted with a single sigmoid (WT A1 $=0.95, \mathrm{Vh} 1=0.03 \mathrm{~V}, \mathrm{~K} 1=0.013, \mathrm{~N}=3$; Eq. 4$)$ and two sigmoids $(\boldsymbol{\Delta} \mathbf{2 - 1 0} \mathrm{A} 1=0.59, \mathrm{Vh} 1$ $=0.0001 \mathrm{~V}, \mathrm{~K} 1=0.013, \mathrm{~A} 2=1.13, \mathrm{Vh} 2=0.049 \mathrm{~V}, \mathrm{~K} 2=0.021, \mathrm{Vh} 3=0.037 \mathrm{~V}, \mathrm{~K} 3=0.032$, $\mathrm{N}=8$; Eq. 5) 
Although TEVC is a powerful technique, we were concerned that the GV changes could be due to an artifact due to the time resolution limitations of TEVC. To test the mutants in a mammalian expression system, HEK293 cells were transfected with $\Delta 2$ 10 and recorded using whole-cell patch-clamp. The kinetics and GV relationship of $\Delta 2$ 10 showed the same differences to wild-type as described in oocytes: kinetics varied across potentials, there was an apparent inactivation at stronger depolarizing potentials, and the normalized end-pulse current showed a biphasic (GV Fig. 8). These results strongly suggest that the effects observed depend on the mutant and are not due to a technical artifact.

\subsubsection{Larger N-terminal deletions and E600R}

The intriguing biphasic GV of $\Delta 2-10$ drove us to generate larger $\mathrm{N}$-terminal deletions, with the expectation that the larger deletions would disturb the intracellular ring. We then examined the GV relationship of the mutants using TEVC recordings. Oocytes were perfused with $60 \mathrm{mM} \mathrm{K}^{+}$solution. We applied increasing voltage steps $(10 \mathrm{mV})$ from $-100 \mathrm{mV}$ to $+120 \mathrm{mV}$ with 30 s interval. A leak subtraction protocol $(\mathrm{P} / \mathrm{n})$ was applied. $-100 \mathrm{mV}$ was used as repolarizing potential.

We noticed a similar behavior of the current to $\Delta 2-10$ when the deletion was extended to residues 2-25 ( $\triangle$ PASCap, Fig. 9). The conductance was calculated using the end-pulse current and the measured reversal potential (Eq. 1, see Methods). The threshold for activation of this mutant was also shifted to hyperpolarized potentials with respect to WT. The conductance increased with stronger depolarization until -20 $\mathrm{mV}$, decreased between -10 and $+50 \mathrm{mV}$, and rose again over this value (Fig. 9E). The tail current amplitude corresponded in this case to the change in end-pulse current across voltage (Fig. 9D). The tail current kinetics was slower than the WT and showed a prominent slow deactivation component up to $+50 \mathrm{mV}$. Then a fast component seemed to be added at stronger depolarizations (see chapter 3.2.1.).

A larger deletion ( $\Delta$ eag, 2-135, Fig. 10) also generated a strong inward rectification after $+10 \mathrm{mV}$. The conductance started decreasing steeply between +10 and $+60 \mathrm{mV}$, increasing slightly with further depolarization. The biphasic behavior was less evident than in the previous mutants, but a small increase in conductance 
appeared with depolarization over $+80 \mathrm{mV}$. Because of the slower and smaller inward currents during the test potential, the conductance presented in the Figure was calculated using tail current (Eq. 2 and Eq.3). There was no major discrepancy between end-pulse and tail current as observed with $\Delta$ eag (end-pulse analysis not shown). The mutant was much slower than WT. We, therefore, use $-160 \mathrm{mV}$ as a repolarizing potential to ensure channel closure.

The mutants described up to now introduce a relatively large change through deletions in the channel. To test if the complex between $\mathrm{N}$-and $\mathrm{C}$-terminal domains is responsible for the biphasic behavior of the mutants, we studied the currents of a single point mutant (E600R, Fig. 11) that disturbs the interface between $\mathrm{N}$ and $\mathrm{C}$ termini (Haitin et al., 2013)Lorinczi, 2016 \#230\}. We used similar voltage protocols as for the other mutants, except that to make sure that the effects observed depend on the voltage applied and not on the sequence of depolarizations, the voltage steps were alternated, and leak subtraction was not performed. We did not detect differences between increasing and alternating voltage steps or introduced by the leak subtraction protocol. The conductance was calculated using the end-pulse current (Eq. 1). E600R also showed early activation and a biphasic behavior, with a decrease in conductance with depolarization between -10 and $+50 \mathrm{mV}$. The tail current amplitude followed the behavior of the end-pulse current. The tail currents in this mutant showed remarkably altered kinetics that will be discussed in chapter 3.2.3.

In summary, in response to depolarizing voltage steps, the $\mathrm{N}$-terminal deletions $(\Delta 2-10, \Delta$ PASCap, $\Delta$ eag) and E600R all respond unusually, with common features but also with clear differences between the mutants. The GV relationship shows an evident rectifying behavior that renders it biphasic to different extents depending on the mutant. The existence of two separable components in the mutants' GV relationship could be explained if we hypothesize that each component corresponds to a different open state. One of the open states would be very unlikely occupied in the WT but favored by deletions or by disrupting the intracellular interactions in the channel molecule. Thus, the WT GV can be fitted reasonably well with a Boltzmann equation (sigmoidal response; Eq. 4). However, there is divergence at strong depolarizations, as it has been repeatedly observed by us and other groups (Schönherr et al., 1999). In the 
rest of the Thesis, we will describe additional evidence supporting this hypothesis and the performance of the model in describing the behavior of the GV of all mutants.

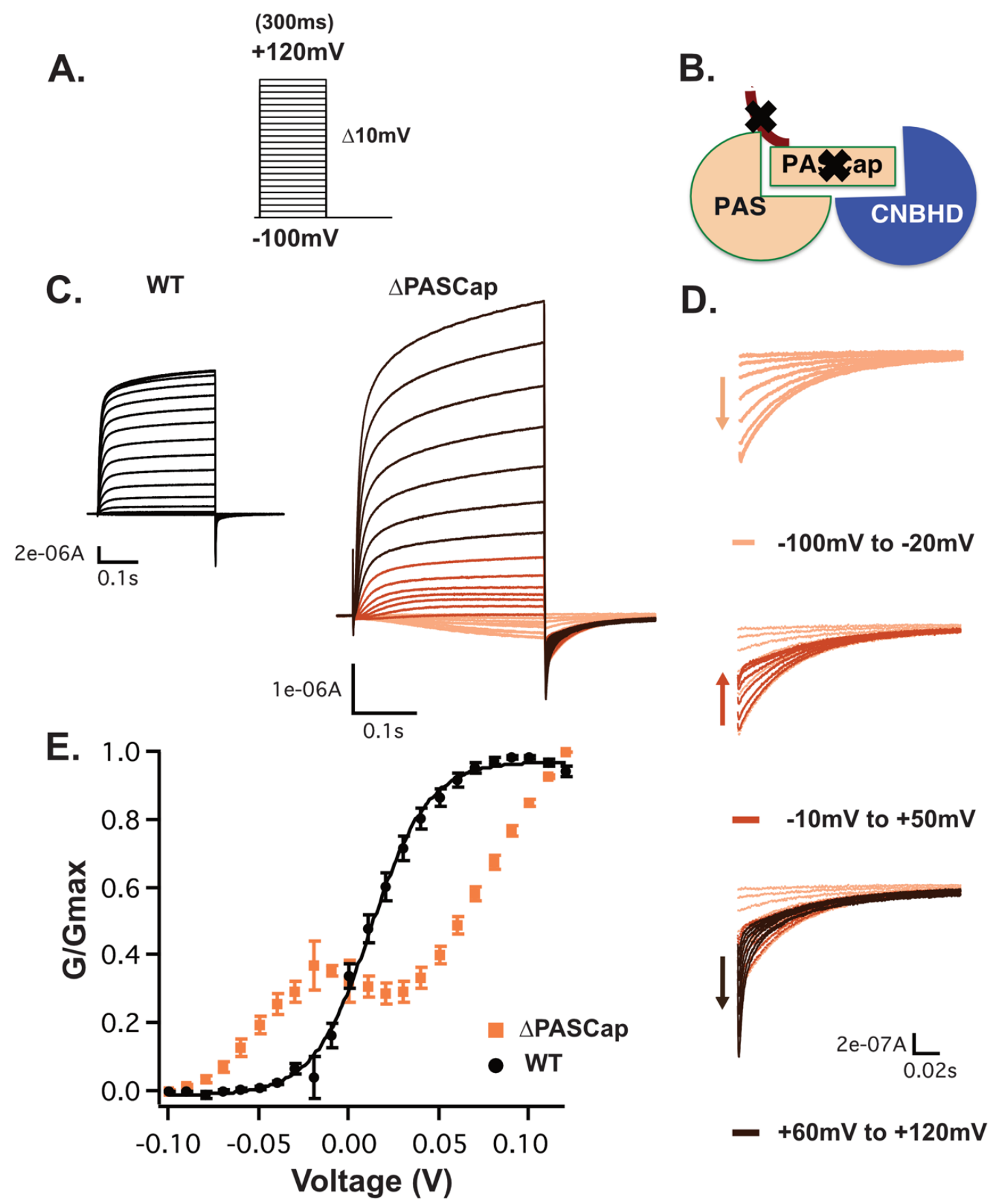

Figure 9. $\triangle$ PASCap unmasks a biphasic GV. A) Voltage protocol applied. Leak subtraction $(\mathrm{P} / \mathrm{N})$ was applied. Recordings were performed in $60 \mathrm{mM} \mathrm{K}^{+}$solution $\mathrm{B}$ ) $\triangle$ PASCap $(\Delta 2-25)$ cartoon C) Representative traces. The color code is the same as shown in D D) Tail current in C. E) Normalized conductance, calculated using end-pulse current (Eq. 1), vs. voltage (N= WT:11, $\triangle$ PASCap:6; mean \pm SEM). WT GV was fitted with a sigmoid: (WT $\mathrm{A}_{1}=0.98, \mathrm{Vh}_{1}=0.012 \mathrm{~V}, \mathrm{~K}_{1}=0.015, \mathrm{~N}=11 ;(E q .4)$ 

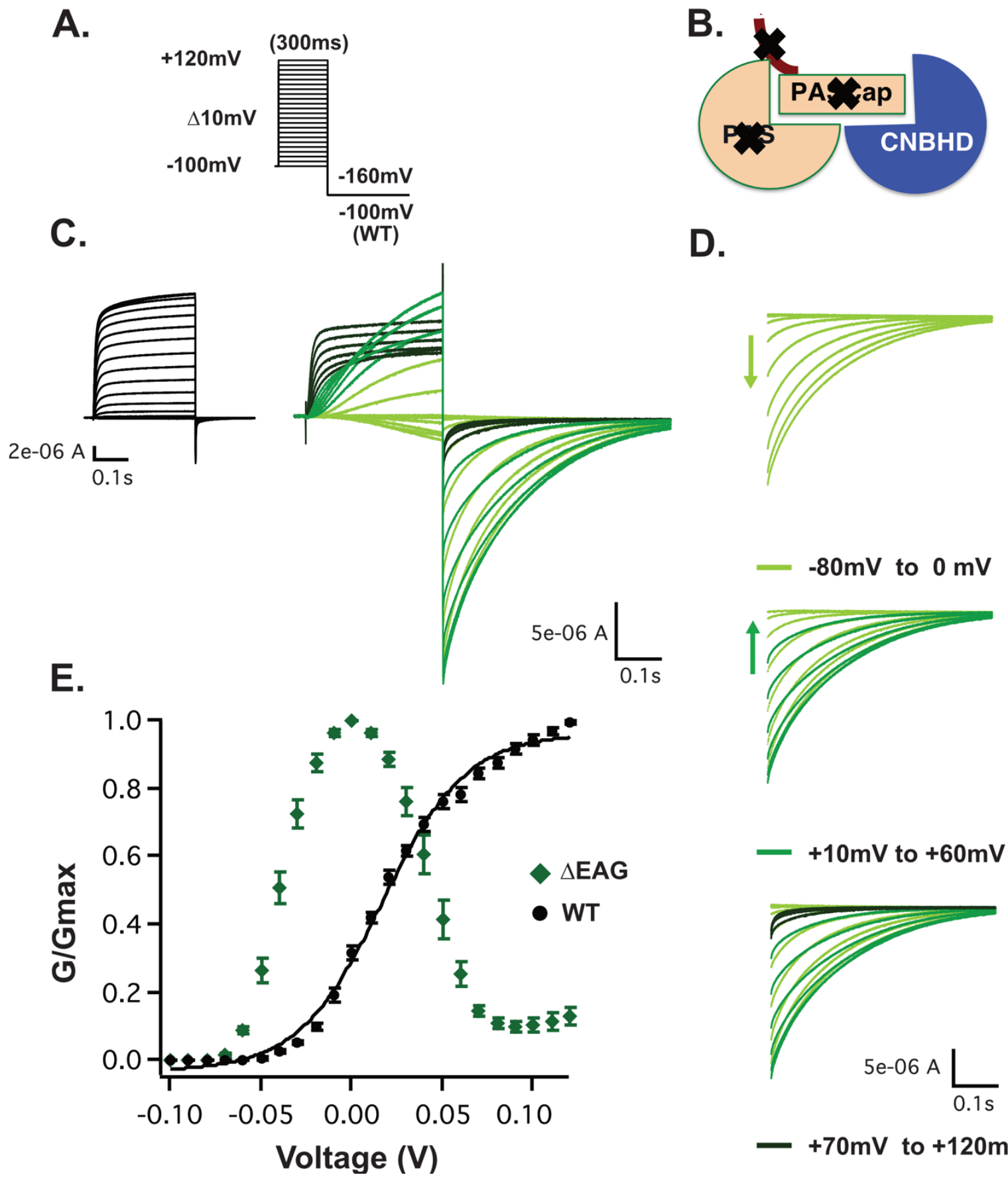

- $+70 \mathrm{mV}$ to $+120 \mathrm{mV}$

Figure 10. $\Delta$ eag reveals a rectifying GV. A) Voltage stimulus used. $\mathrm{P} / \mathrm{N}$ protocol was used for leak subtraction. Recordings were performed in $60 \mathrm{mM} \mathrm{K}^{+}$solution. B) $\Delta \mathrm{eag}$ cartoon C) Representative traces. The color code is same as shown in D. D) Tail current in C. E) Normalized conductance, deduced from tail current (Eq. 2, Eq. 3), vs. voltage (N: $\mathrm{WT}=9 ; \Delta \mathrm{eag}=6$; mean $\pm \mathrm{SEM}$ ). GV was fitted using single sigmoid (WT $\mathrm{A}_{1}=0.99$, $\mathrm{Vh}_{1}=0.016 \mathrm{~V}, \mathrm{~K}_{1}=0.023 ; E q .4$ ). 


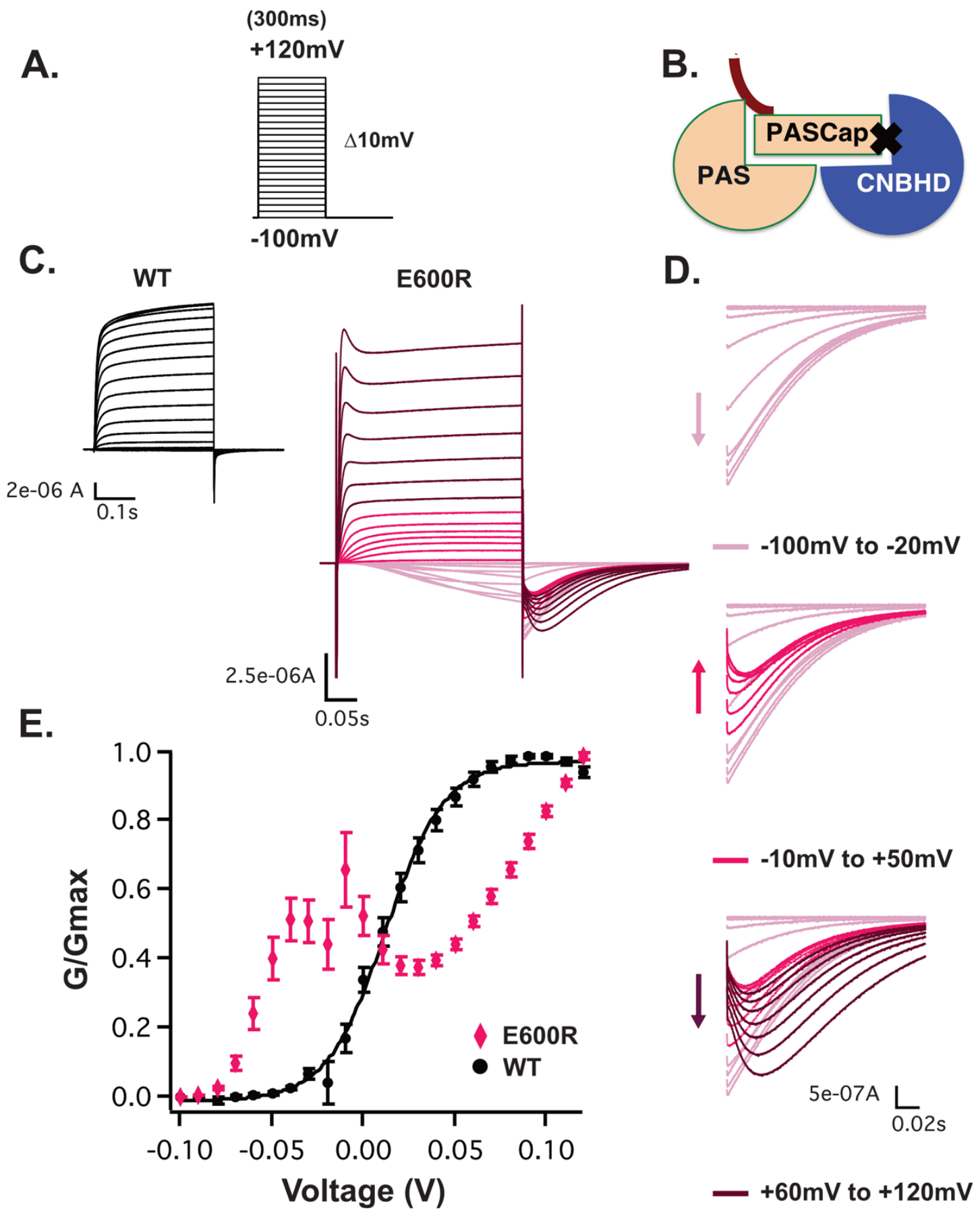

Figure 11. E600R replicates the biphasic behavior observed with $\mathrm{N}$-terminal deletions. A) Voltage stimulus; Voltage steps were alternated. Recordings were performed in $60 \mathrm{mM} \mathrm{K}^{+}$solution without leak subtraction. B) E600R cartoon C) Representative current response. The color code is same as shown in D. D) Tail current in C. E) Normalized conductance was plotted against voltage (N: WT=11; E600R=6 mean \pm SEM): GV. G was calculated using end pulse current (Eq. 1). GV was fitted with a one-sigmoid curve (WT: $\mathrm{A}_{1}=0.98, \mathrm{Vh}_{1}=0.012 \mathrm{~V}, \mathrm{~K}_{1}=0.015 ; E q .4$ ) 


\subsubsection{Biphasic kinetic behavior in N-terminal deletions and E600R}

The activation and deactivation kinetics vary across different potentials. Like other voltage-gated channels, the activation of WT channels is mildly accelerated with increasing depolarization, and the speed of deactivation remains constant with equally long depolarizations and constant potential for repolarization. Neither of the two features is maintained in the mutants. To navigate across the different mutants, we classified the voltage steps into three categories:

- $\quad$ weak $(\Delta 2-10$ and $\Delta$ eag: below $+10 \mathrm{mV}$, E600R and $\Delta$ PASCap: below $-10 \mathrm{mV})$

- moderate $(\Delta 2-10$ and $\Delta$ eag: $+10 \mathrm{mV}$ to $+60 \mathrm{mV}$ )

(E600R and $\triangle$ PASCap: $-10 \mathrm{mV}$ to $+50 \mathrm{mV}$ )

- strong $(\Delta 2-10$ and $\Delta$ eag: more than +60 , E600R and $\Delta$ PASCap: more than +50$)$ depolarization steps.

The voltage steps were classified based on the biphasic GV (see chapter 3.2.1 and 3.2.2); the first component appeared with weak depolarizations, while the second component prevailed at strong depolarization. The transition between the two components was seen as a rectifying behavior at moderate potentials.

To visualize the change in activation kinetics, the current traces in Figs. 6 and 911 were normalized to the last $20 \mathrm{~ms}$ of the depolarizing pulse (300ms). Representative traces of each voltage range were superimposed and depicted in Fig. 12. The time required to reach $80 \%$ of the maximum current (Risetime $80 \%$ ) was used to quantify the change in activation over the voltage range $(-40 \mathrm{mV}$ to $+120 \mathrm{mV})$.

There was no drastic change in the WT activation kinetics across the three ranges; moderate $(+60 \mathrm{mV})$ and strong potentials $(+100 \mathrm{mV})$ overlapped and could not be discriminated, while weak depolarization $(0 \mathrm{mV})$ was slightly slower. Risetime $80 \%$ confirmed this observation as it was similar across the voltage range (WT: $-40 \mathrm{mV}=$ $0.020 \pm 0.0043 \mathrm{~s},+100 \mathrm{mV}=0.019 \pm 0.002 \mathrm{~s} ;$ mean $\pm \mathrm{SEM})$.

In contrast to WT, the activation kinetics of the mutants changed significantly across potentials. $\Delta 2-10$ showed slow sigmoidal activation in response to weak potentials, which accelerated as the depolarization increased to moderate and strong potentials. 
In addition to the acceleration, inactivation was visible with moderate and strong depolarizing pulses (Fig. 12. A). Risetime80\% was larger than in WT at weak potentials, then it decreased as the potential increased until it reached equivalent kinetics to WT at approximately $+60 \mathrm{mV}$ (strong potentials) $(\Delta 2-10:-40 \mathrm{mV}=0.19 \pm 0.014 \mathrm{~s},+100 \mathrm{mV}=$ $0.02 \pm 0.002 \mathrm{~s} ;$ mean \pm SEM) (Fig. 12B).

$\triangle$ PASCap followed a similar activation pattern: slow sigmoidal activation at weak potentials that became faster with moderate and strong potentials. However, moderate and strong potentials almost overlapped, and inactivation was not visible within a 300ms pulse. Moderate and strong potentials showed similar kinetics to WT; a slow rising activation phase could be observed (Fig. 12C). Risetime80\% was high at weak potentials and became smaller at moderate and strong potentials (starting at $0 \mathrm{mV}$ ) (APASCap: $-40 \mathrm{mV}=0.17 \pm 0.007 \mathrm{~s},+100 \mathrm{mV}=0.055 \pm 0.007 \mathrm{~s} ;$ mean \pm SEM) (Fig. 12D).

$\Delta$ eag representative traces also showed acceleration from weak to strong potentials. Intriguingly, the trace at $+60 \mathrm{mV}$ (moderate potential) showed a combination of fast and slow activation (Fig. 12E). Risetime80\% was large at weak potentials and got faster starting $+50 \mathrm{mV}$ (the end of the moderate potential) until it reached similar values to the $\mathrm{WT}$ at strong potentials $(\Delta \mathrm{eag}:-40 \mathrm{mV}=0.22 \pm 0.004 \mathrm{~s}$, $+100 \mathrm{mV}=0.027 \pm 0.0055 \mathrm{~s}$; mean \pm SEM) (Fig. 12F).

In addition to the familiar pattern of accelerating the activation kinetics with stronger potentials, E600R showed two phases of activation at strong potentials: a fastactivating component and a slow activating component. The fast component seemed to inactivate, which allowed discrimination between the two phases (Fig. 12G). A similar pattern of risetime $80 \%$ was observed; it reached WT values around $+50 \mathrm{mV}$ (moderate and strong potentials). (E600R: $-40 \mathrm{mV}=0.20 \pm 0.004 \mathrm{~s},+100 \mathrm{mV}=0.014 \pm$ 0.006s; mean \pm SEM) (Fig. 12H). 
A.

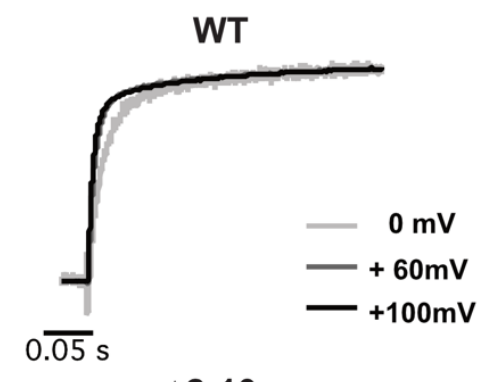

$\Delta 2-10$

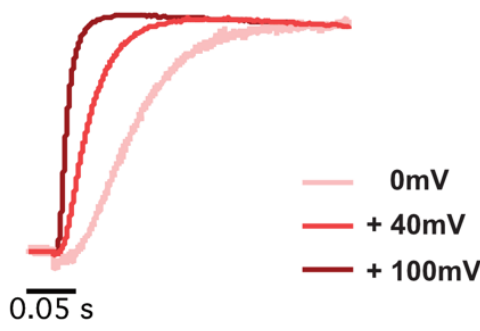

$\triangle$ PASCap

C.

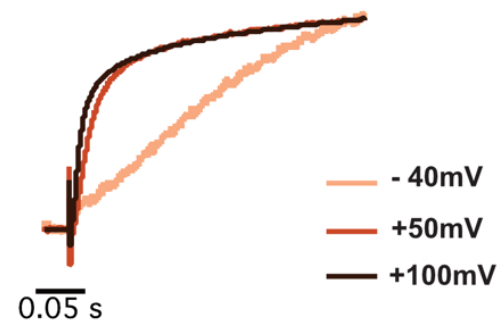

E

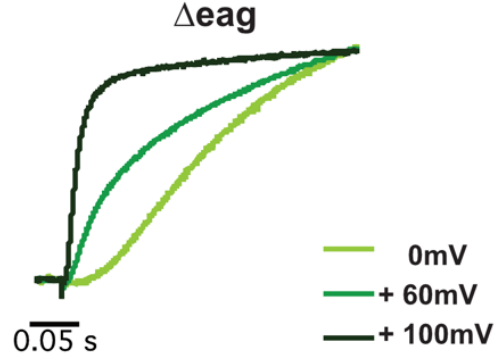

G.

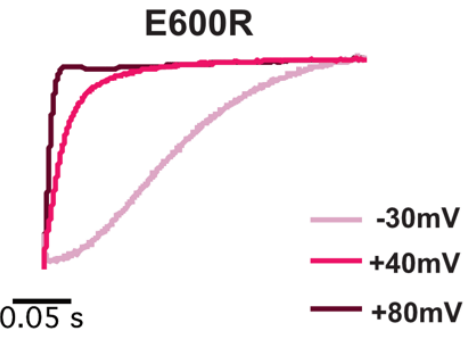

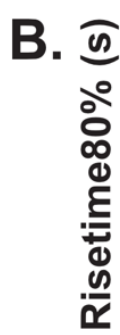

$0.20-t^{-\mathbf{F}^{\mathbf{z}} \mathbf{I}_{\mathbf{I}}}$

$\nabla \Delta 2-10$

- WT

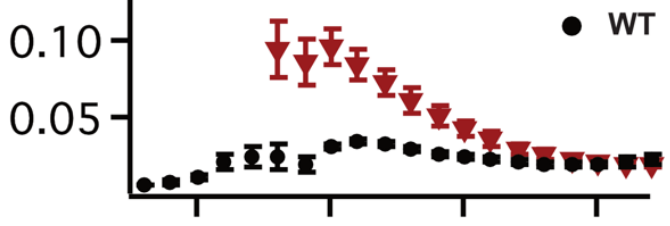

$-50$

Voltage $(\mathrm{mV})$

D. ฮี

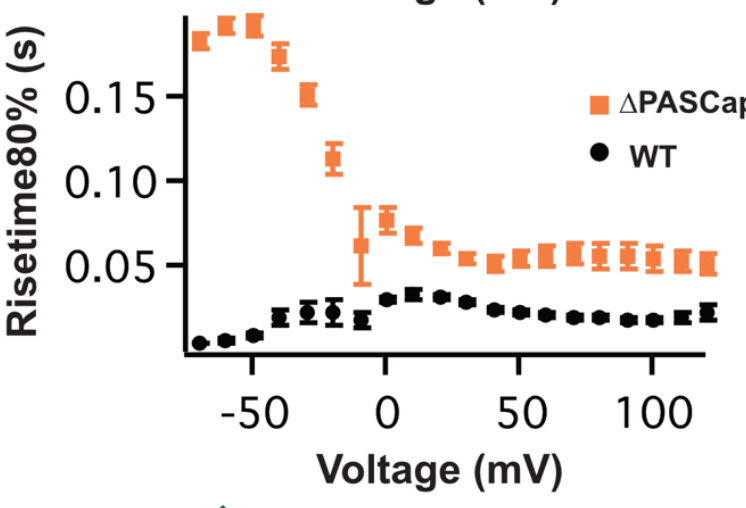

F. $\stackrel{\bar{m}}{0} 0.20 \mathrm{f}^{\mathrm{x}^{*}}=$

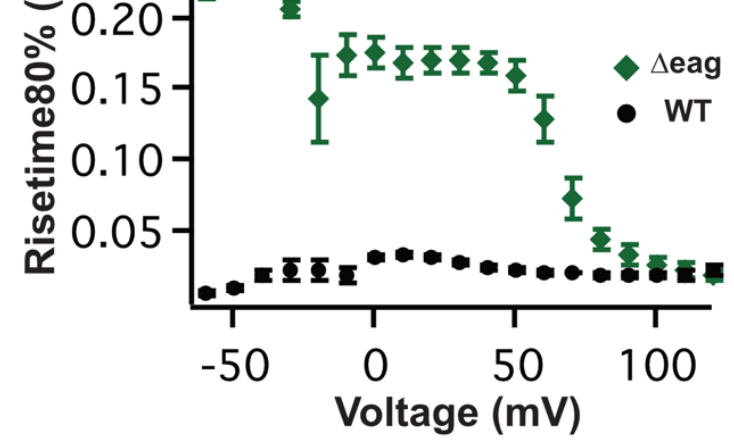

H.

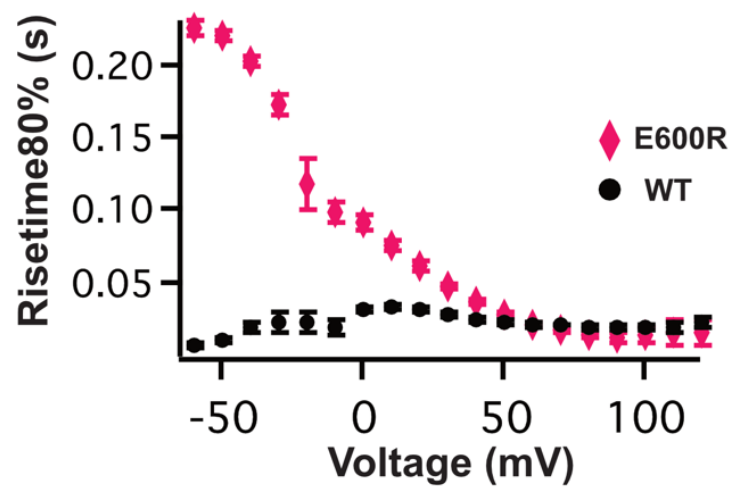

Figure 12. Activation kinetics of $\mathrm{N}$-terminal deletions and E600R accelerates with stronger potentials. Normalized current traces: A, B) WT, $\Delta 2-10$ C, D) $\triangle$ PASCap E, F) $\Delta$ eag G, H) E600R. B, D, F, H) Risetime $80 \%$ was plotted against voltage (mean \pm SEM): WT, N=9, B) $\Delta 2-10, \mathrm{~N}=9 \mathrm{D}) \Delta \mathrm{PASCap}, \mathrm{N}=5$ F) $\Delta$ eag, $\mathrm{N}=6$ H) E600R, $\mathrm{N}=10$ 
The tail current kinetics of the mutants were quite intriguing. Figure 13 (left panels) shows representative traces obtained at the three different ranges (weak, moderate, and strong), and tail currents normalized to the largest absolute value of inward current (right panel). Tail currents shown in Fig. 13 are the same as those in Figs. 6 and $9-11$

$\Delta$ 2-10 tail currents showed a rectifying behavior; the amplitude was largest when stepping from weak potentials $(0 \mathrm{mV})$ and decreased with stronger depolarizations $(+60$ and $+100 \mathrm{mV})$. The normalized tail currents showed a slight acceleration of the deactivation kinetics coming from stronger depolarization. At strong depolarizations, at least two components could be observed. The tail currents shown are in response to $-160 \mathrm{mV}$ repolarizing pulse. The kinetics were slower and more complex at $-100 \mathrm{mV}$ (data not shown).

$\triangle$ PASCap tail current amplitudes from $-20 \mathrm{mV}$ and $+100 \mathrm{mV}$ were the same, while it was smaller at $+50 \mathrm{mV}$. This reflects the biphasic GV in Fig. 9D; the tail current grew in response to weak depolarizations $(-20 \mathrm{mV})$, then decreased with moderate potentials $(+50 \mathrm{mV})$, and another component increased at strong potentials $(+100 \mathrm{mV})$. The tail currents shown are during $-100 \mathrm{mV}$ repolarizing potential. The kinetics were also different; a slow component was seen at weak potentials $(-20 \mathrm{mV})$, while a faster component was observed at stronger depolarizations and $+100 \mathrm{mV}$ (strong potential), while the moderate potential showed two components (fast and slow).

$\Delta$ eag tail currents showed a rectifying behavior similar to the $\Delta 2-10$; the largest amplitude at weak potentials, followed by a decline at moderate and strong potentials. Tail currents shown are in response to $-160 \mathrm{mV}$. Normalized tail currents show an acceleration pattern from weak to strong potentials.

E600R tail current amplitude followed a similar pattern to $\triangle$ PASCap. Tails shown correspond to repolarization to $-100 \mathrm{mV}$. A slow deactivation component dominated at weak potentials. As the potential increased (moderate and strong potentials), a rising phase appeared. This rising phase was also observed with $\Delta 2-10$ and $\Delta$ eag when using $-100 \mathrm{mV}$ repolarizing potential (data not shown). 
In summary, the biphasic GV and kinetics of N-terminal deletion mutants indicate the existence of at least two different open states, reached with different kinetics and voltage dependence. The first component with slow activation and deactivation kinetics would correspond to a state almost not detected in WT, that we will refer to as $\mathrm{O}_{1}$. The second component, with fast activation and deactivation kinetics, would reflect the dominant state in $\mathrm{WT}\left(\mathrm{O}_{2}\right)$. 
A.
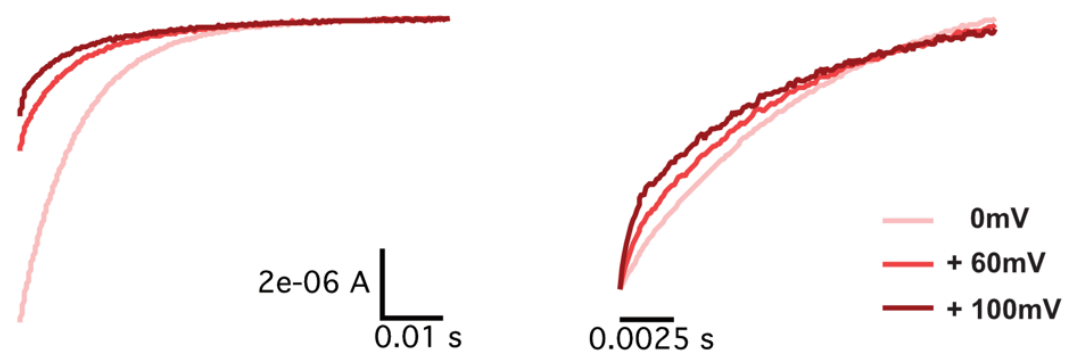

B.

$\triangle$ PASCap
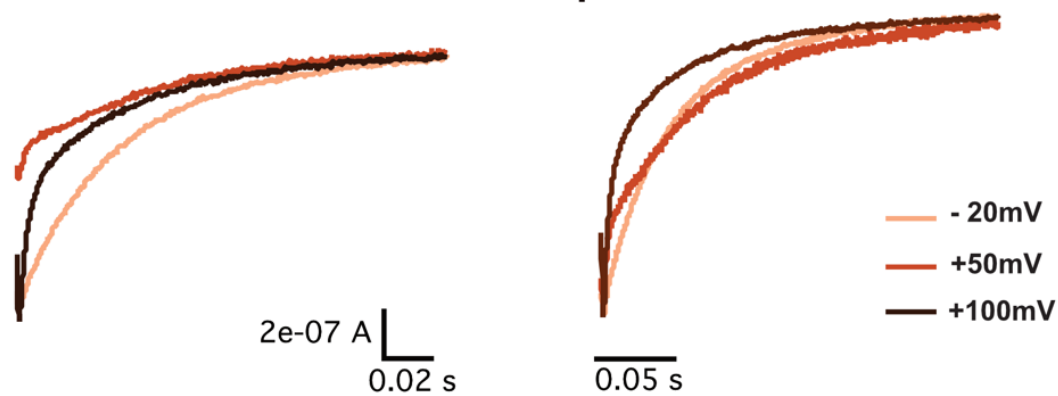

C.

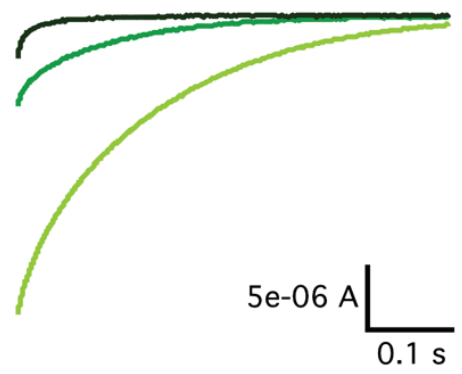

$\Delta$ eag

D.

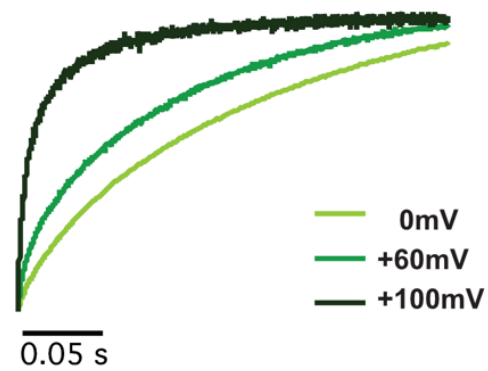

\section{E600R}
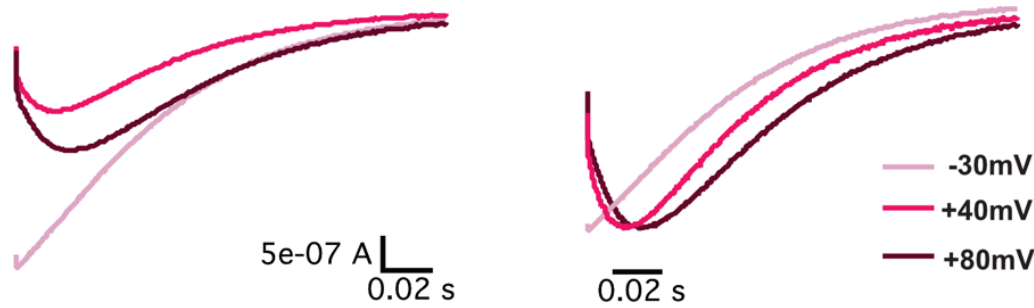

Figure 13. N-terminal deletions and E600R show peculiar deactivation kinetics. Representative tails(left), Normalized tails (right) A) $\Delta 2-10$. B) $\Delta$ PASCap. C) $\Delta$ eag. D) E600R.

\subsection{4 $\mathrm{O}_{1}$ probably has higher conductance than $\mathrm{O}_{2}$}

In Fig. 6C, $\Delta 2-10$ showed inactivation at strong and moderate potentials during $300 \mathrm{~ms}$ depolarizing pulses. We, therefore, set out to design a set of experiments to examine this apparent inactivation. We first applied longer depolarizing pulses (2s). 
Inactivation was seen at weak, moderate, and strong potentials (Fig. 14). We next examined the voltage dependence of inactivation. We applied pre-pulse potentials from $-160 \mathrm{mV}$ to $+80 \mathrm{mV}$ in $20 \mathrm{mV}$ increments, followed by a test pulse of $+100 \mathrm{mV}$ (Fig. 14C, D). If channels inactivate during the pre-pulse, a decline in current amplitude at the test pulse is expected since inactivated channels will not have had time to deactivate. The current at the end of the test pulse was normalized to the maximum response. In contrast to WT, $\Delta 2-10$ current declined with depolarizing pre-pulses, strongly suggesting inactivation. The decline started at $-80 \mathrm{mV}$ and saturated around $+20 \mathrm{mV}$ (Fig. 14E). This range of potentials corresponds to the weak depolarizing potentials defined above.

We also examined $\Delta 2-10$ inactivation in more detail with a set of pre-pulses with variable duration. The potential of the pre-pulse was held constant, and the duration was changed from $200 \mathrm{~ms}$ to $5 \mathrm{~s}$. If inactivation occurred during the pre-pulse, then a longer duration would cause a decline in the test potential. We tested the following pre-pulse potentials: $-120 \mathrm{mV},-100 \mathrm{mV},-80 \mathrm{mV},-60 \mathrm{mV},-40 \mathrm{mV}$ and $-20 \mathrm{mV}$, and a test potential of $+40 \mathrm{mV}$ was used. The current at the end of the pulse was normalized to the shortest pulse $(200 \mathrm{~ms})$. A pre-pulse of $-20 \mathrm{mV}$ showed the largest decrease, followed by $-40 \mathrm{mV},-60 \mathrm{mV}$ and $-80 \mathrm{mV}$ \%decline: $-20 \mathrm{mV}=43 \% \pm 0.06$; $40 \mathrm{mV}=40 \% \pm 0.04 ;-60 \mathrm{mV}=33 \% \pm 0.03 ;-80 \mathrm{mV}=22 \% \pm 0.02)$. No decline occurred at $100 \mathrm{mV}$ and $-120 \mathrm{mV}$ (Fig. 15A). The potentials (-80, -60, -40 , and $-20 \mathrm{mV}$ ) match with the range of potentials identified with the previous set of experiments $(-80 \mathrm{mV}$ to $+20 \mathrm{mV}$, Fig. 14). The response to $-20 \mathrm{mV}$ pre-pulse showed an intriguing feature. There was an initial peak at the beginning of the pulse, which will be discussed below. As a control for this set of experiments, a similar set was designed where the duration was held constant (3s), and the same pre-pulse potentials were tested (Fig. 16). This control rules out the impact of stimulus repetition on the current amplitude. Only a slight decrease was observed, which supports our observations with variable pulse duration.

Longer pulse protocols were then designed for other mutants to visualize apparent inactivation during the pulse. $\triangle$ PASCap showed apparent inactivation at moderate depolarizations when 2s depolarizing pulses were applied (Fig 17C). Recordings were performed in a $60 \mathrm{mM} \mathrm{K}^{+}$solution. Conductance was calculated using the tail current (Eq. 2 and Eq.3). In comparison to $300 \mathrm{~ms}$ depolarizing pulses, 2 s pulses attenuated the 
first component of the characteristic biphasic GV. E600R also showed inactivation at moderate and strong depolarizations when a depolarizing pulse of $1 \mathrm{~s}$ was used. Recordings were performed in NFR solution (Fig. 18).

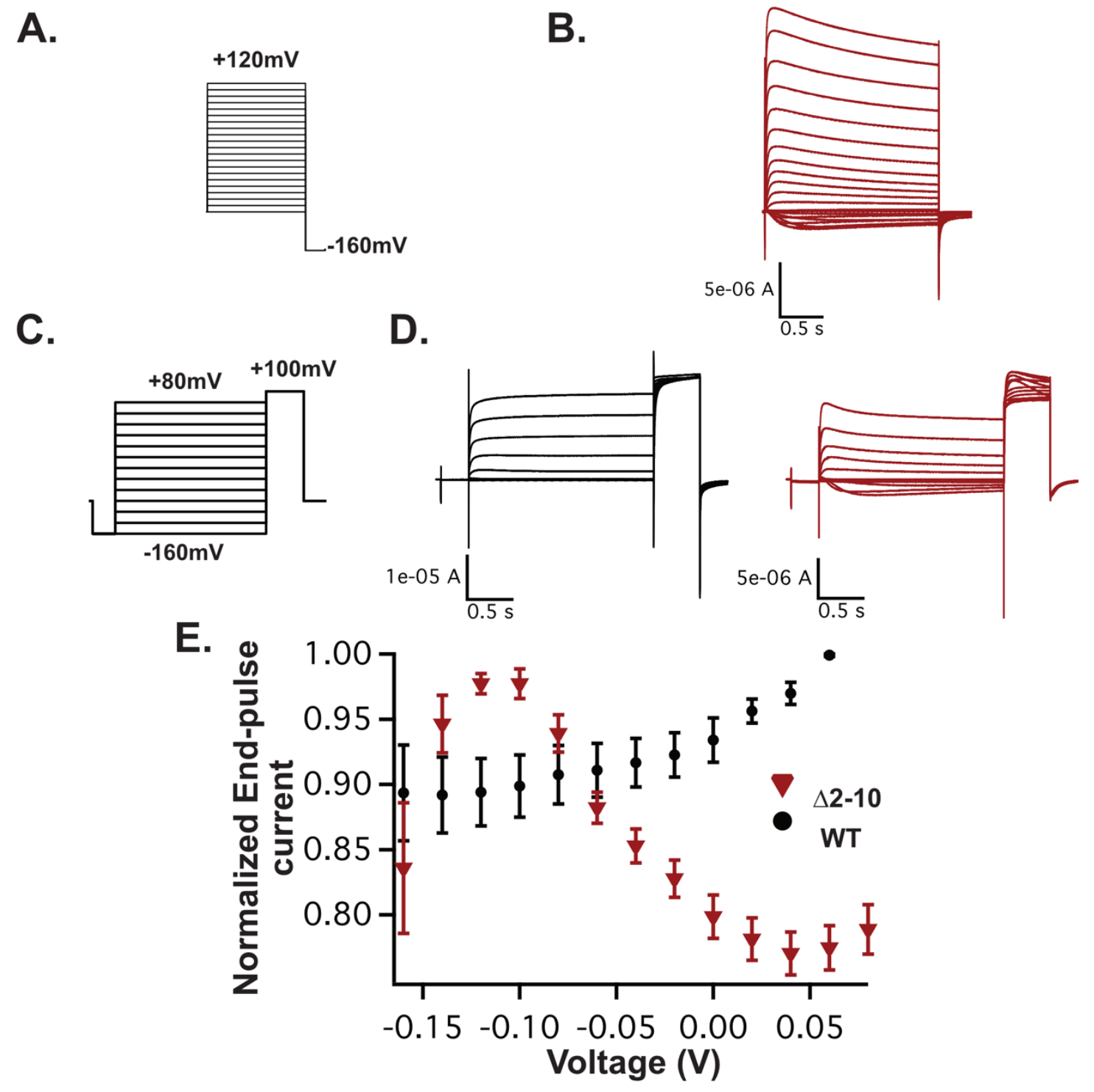

Figure 14. Apparent inactivation is evident with longer pulse duration ( $\Delta 2-10)$. Recordings were performed in $60 \mathrm{mM} \mathrm{K+}$. A). Voltage protocol (2 s pulse). B) $\Delta 2-10$ current response. C). Voltage protocol. D). Representative current traces: WT and $\Delta 2$ 10 E). Normalized end pulse current vs. pre-pulse potential $(\Delta 2-10 \mathrm{~N}=8$, WT $N=6$; Mean \pm SEM) 
A.
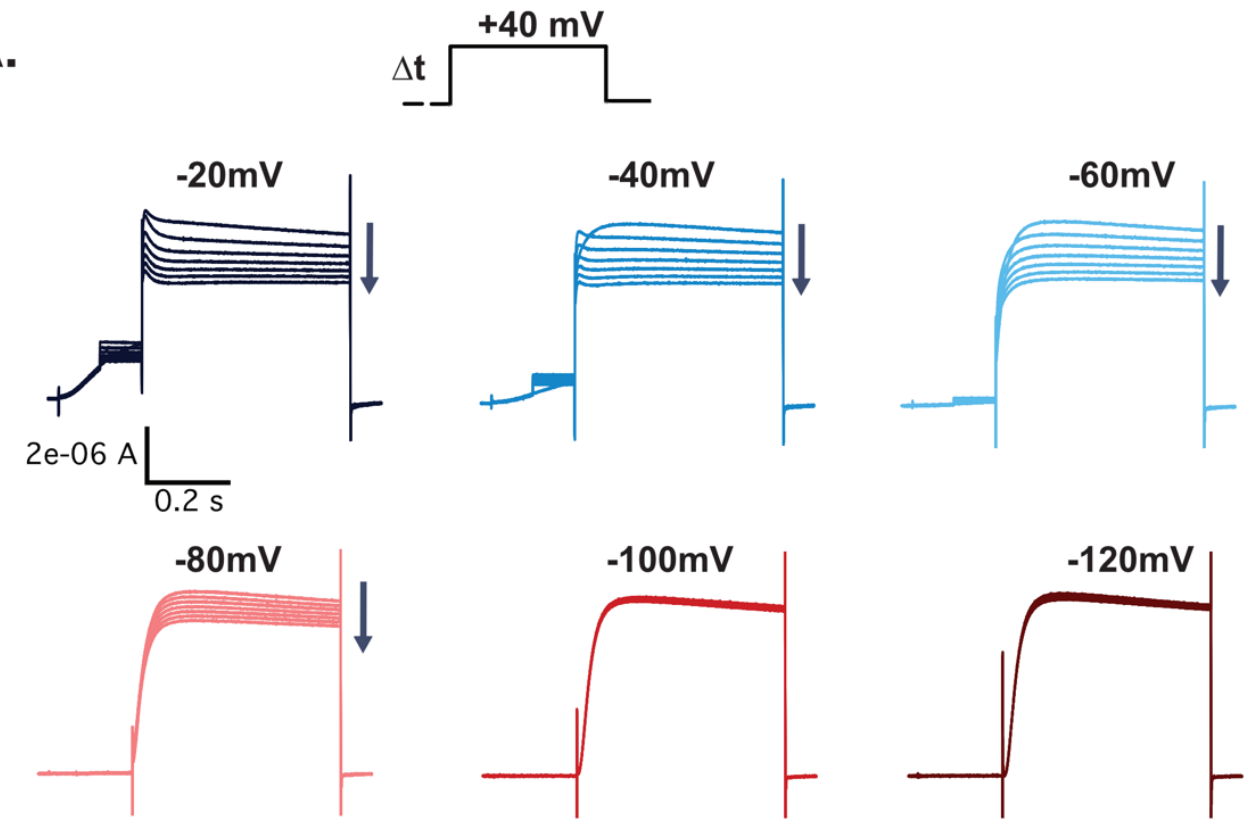

2e-06 A $\frac{}{0.2 \mathrm{~s}}$

B.

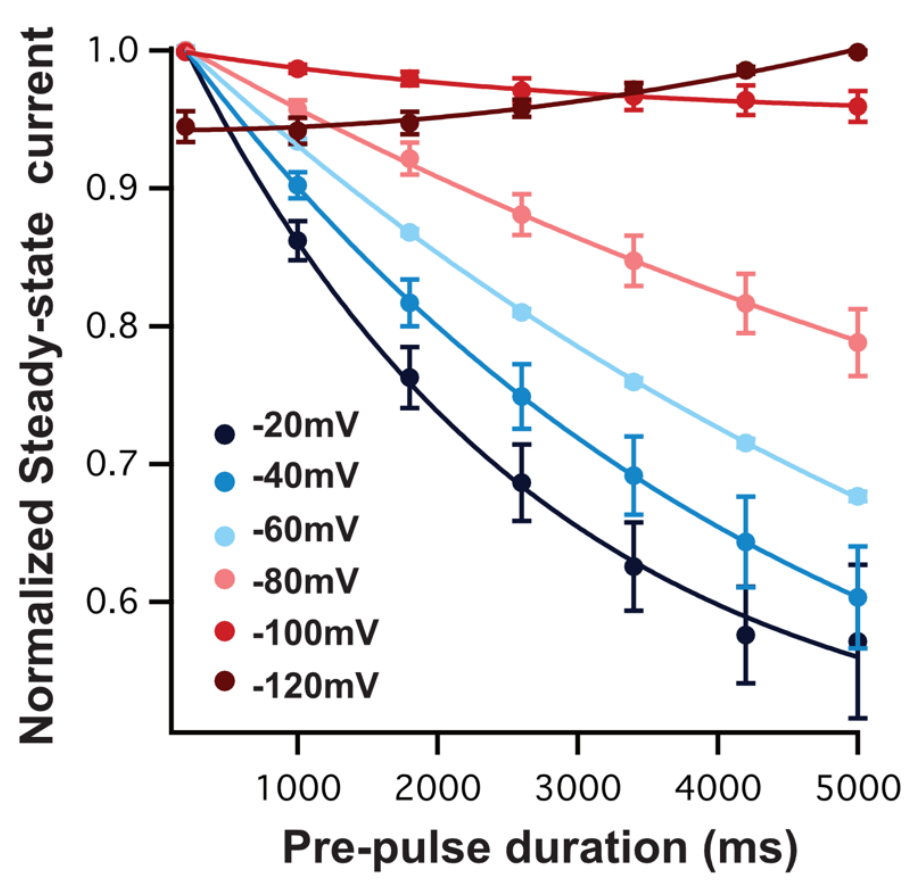

Figure 15. Apparent inactivation is confirmed with varying pre-pulse potentials $(\boldsymbol{\Delta} \mathbf{2 - 1 0})$. Recordings were performed in NFR. A) Voltage protocol; A fixed pre-pulse voltage was applied for variable duration (200ms to $5 \mathrm{sec}, 800 \mathrm{~ms}$ step, $20 \mathrm{~s}$ interval). B) Representative recordings. C) Normalized steady-state current vs. pre-pulse duration. $(-20 \mathrm{mV}, \mathrm{N}=10 ;-40 \mathrm{mV}, \mathrm{N}=11 ;-60 \mathrm{mV}, \mathrm{N}=13 ;-80 \mathrm{mV}, \mathrm{N}=11 ;-100 \mathrm{mV}, \mathrm{N}=12$; $-120 \mathrm{mV}, \mathrm{N}=12$; Mean $\pm \mathrm{SEM}$ ) 


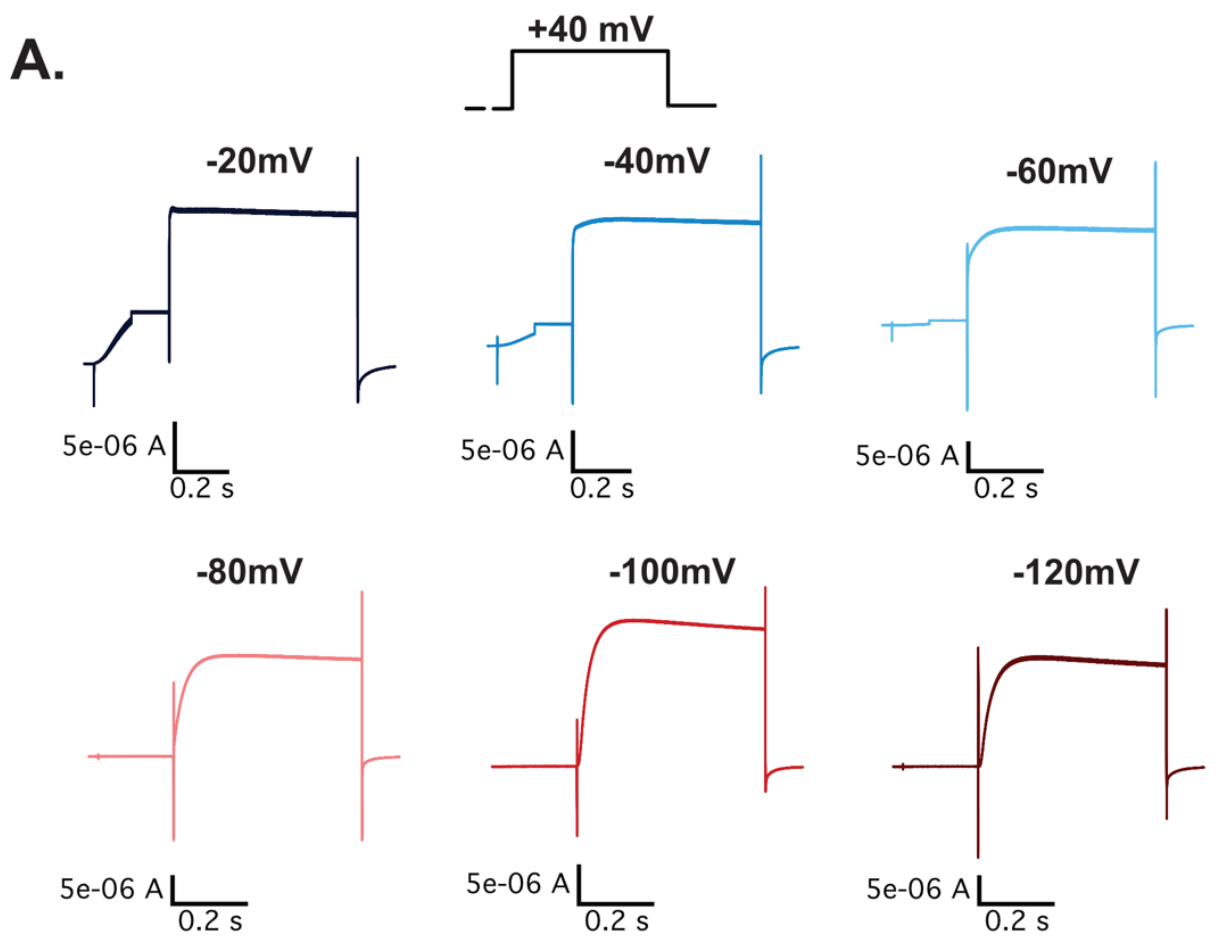

B.

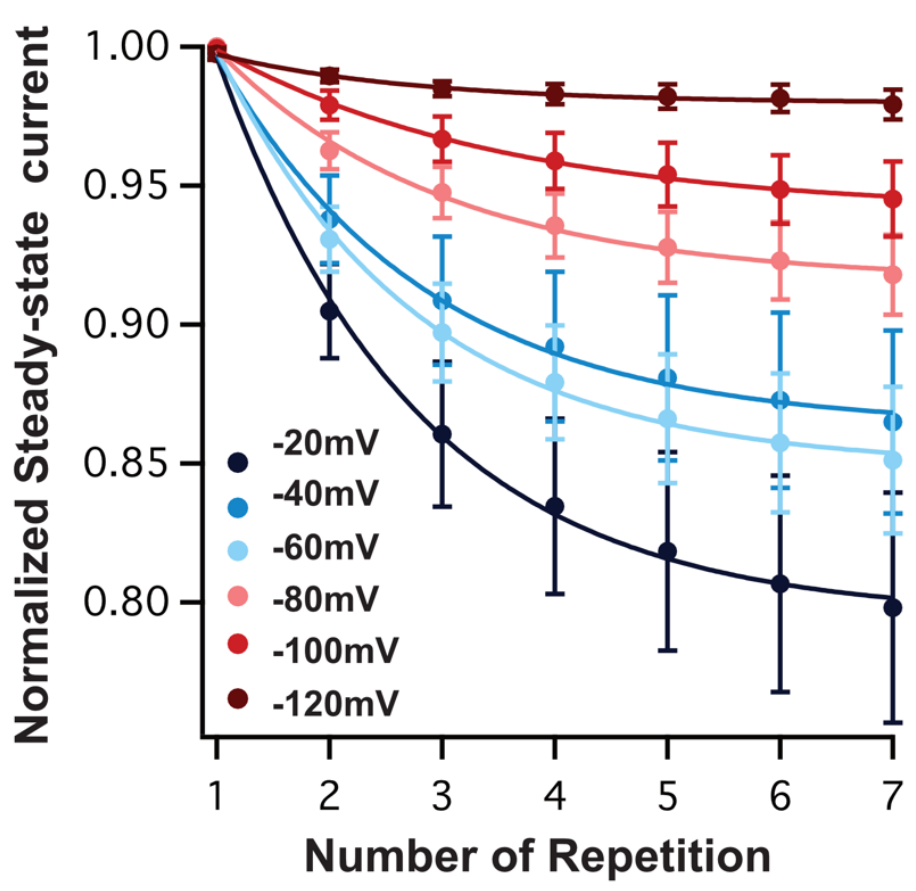

Figure 16. Repetition of pre-pulse voltage with fixed duration causes a slight decrease in current amplitude. Recordings were performed in NFR. A) Voltage protocol. A fixed pre-pulse voltage was applied for 3 sec. B) Representative recordings. C) Normalized steady-state current vs. pre-pulse repetition. $(-20 \mathrm{mV}, \mathrm{N}=8 ;-40 \mathrm{mV}, \mathrm{N}=9$; $-60 m V, N=10 ;-80 m V, N=10 ;-100 m V, N=10 ;-120 m V, N=10 ;$ Mean \pm SEM). 


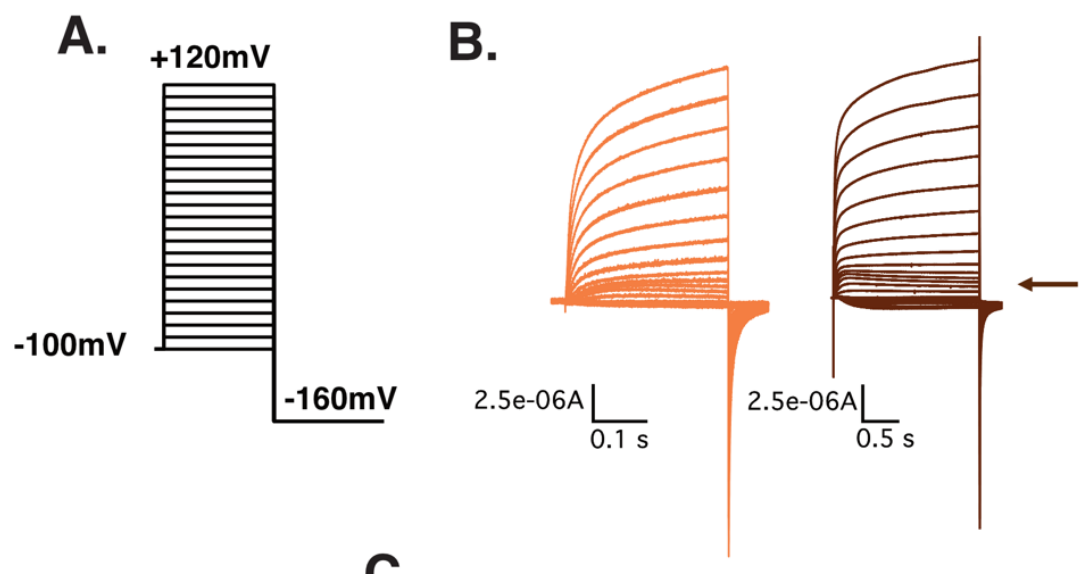

C.

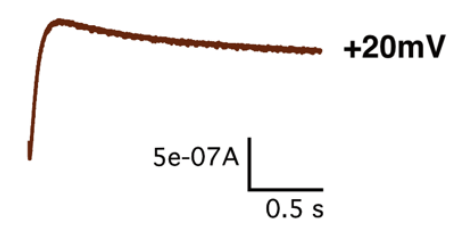

D.

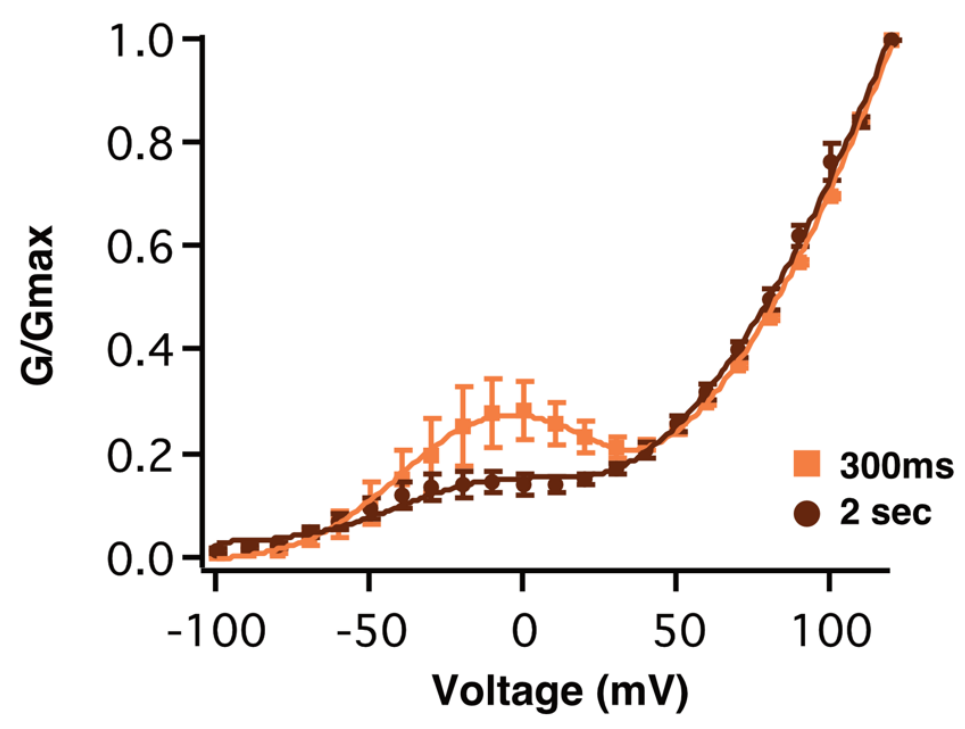

Figure 17. Apparent inactivation is observed with moderate depolarizing pulses (APASCap). A) Voltage steps applied for different durations (300 ms, $2 \mathrm{~s}$ ). P/N protocol was used for leak subtraction. Oocytes were perfused with $60 \mathrm{mM} \mathrm{K}+$ solution. B) Representative traces. C) Inactivation during moderate depolarization (+20mV) D) Normalized conductance, deduced from tail current Eq. 2 and Eq. 3, vs. voltage. Global fit of the GV was performed using Eq. 5 (Linked parameters (300ms and 2s): Vh1= $40.7 \mathrm{mV}, \mathrm{K} 1=15.8, \mathrm{Vh} 2=125.6 \mathrm{mV}, \mathrm{K} 2=36, \mathrm{Vh} 3=25 \mathrm{mV}, \mathrm{K} 3=12 ; 300 \mathrm{~ms}: \mathrm{A} 1=0.33, \mathrm{~A} 2=$ 2.15; 2s: $\mathrm{A} 1=0.14, \mathrm{~A} 2=2.12 \mathrm{~N}=8 ; \pm \mathrm{SEM}$ ) 
A.

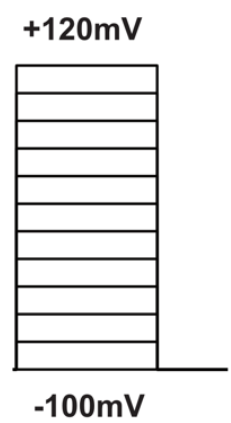

B.

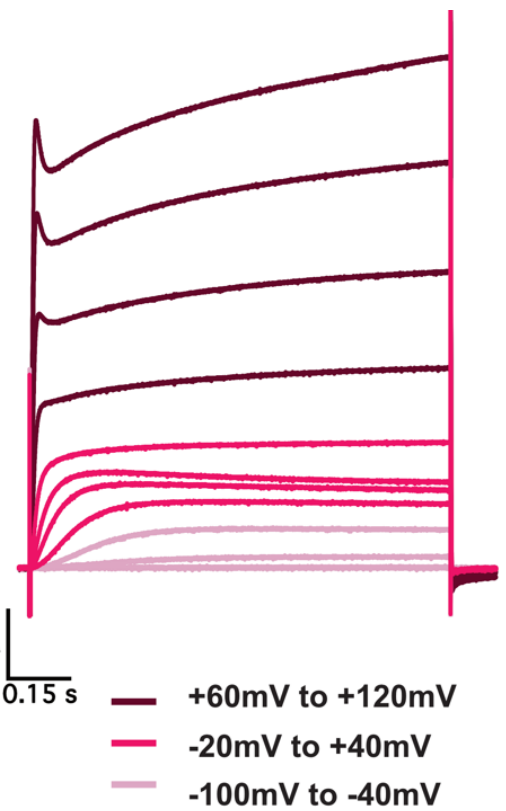

Figure 18. E600R show apparent inactivation at moderate and strong depolarizations. A) Voltage stimulus (1s pulse). Recording performed in NFR solution. B) E600R current response.

In light of the two-state hypothesis $\left(\mathrm{O}_{1}\right.$ and $\left.\mathrm{O}_{2}\right)$, the apparent inactivation could reflect a transition between two states with different conductance. This possibility could also explain the rectifying behavior of the mutants (see above); stronger depolarizations could show smaller tail current amplitude. We, therefore, predicted that $\mathrm{O}_{1}$ has higher conductance than $\mathrm{O}_{2}$. To test this prediction, we designed a protocol with alternating voltage pulses (Square pulses). The voltage alternated between weak and strong potentials, where $\mathrm{O}_{1}$ and $\mathrm{O}_{2}$ were expected to dominate, respectively. Alternating the voltage could result in a population mix of both states. Therefore, the rate of activation and the total conductance would be influenced by both states. If $\mathrm{O}_{1}$ had a higher conductance, then a higher current amplitude would be expected at strong depolarizations compared to a constant pulse.

We applied square pulse protocol to the mutants: $\Delta 2-10, \Delta$ PASCap, $\Delta$ eag, and E600R. $\Delta$ eag and E600R showed the most intriguing response. The voltage was alternated at $50 \mathrm{~Hz}$ between +80 and $-80 \mathrm{mV}$ for $300 \mathrm{~ms}$ (E600R, Fig. 19B) and 500ms ( $\Delta$ eag, Fig. 19C). Therefore, the channels spent $10 \mathrm{~ms}$ at each potential. Equivalent constant pulses $(-80$ and $+80 \mathrm{mV})$ were applied for the same duration as the square pulses. The current response to square pulses is shown in black, while the response to constant pulses is colored with the same code of the respective mutant. Recordings 
were performed in $60 \mathrm{mM} \mathrm{K} \mathrm{K}^{+}$solution, and a repolarizing pulse of $-100 \mathrm{mV}$ was used. At $+80 \mathrm{mV}, \mathrm{E} 600 \mathrm{R}$ and $\Delta$ eag showed a tail current with a rising phase and almost no tail current at $-80 \mathrm{mV}$. As predicted, the current amplitude in response to square pulses was larger than the current amplitude at a constant potential $(+80 \mathrm{mV})$. The tail current increased as well, and the kinetics changed; the rising phase was missing. We attribute this increase to populating $\mathrm{O}_{1}$. At $-80 \mathrm{mV}$, square pulses also showed more current than constant pulses. During $10 \mathrm{~ms}$ of both $+80 \mathrm{mV}$ and $-80 \mathrm{mV}$, the current increased (Fig.19B and D), indicating that $-80 \mathrm{mV}$ opened the channel instead of closing it as in WT (see below).

In contrast to E600R and $\Delta \mathrm{eag}, \Delta 2-10$ and $\triangle \mathrm{PASCap}$ did not show a remarkable increase in current amplitude. Similar square pulse protocols were applied, and recordings were performed in a $60 \mathrm{mM} \mathrm{K}^{+}$solution. The voltage was alternated between +80 and $-80 \mathrm{mV}$ with $50 \mathrm{~Hz}$ for $500 \mathrm{~ms}(\Delta 2-10$, Fig. 20$)$. The current amplitude in response to $+80 \mathrm{mV}$ (Square pulses) was either less than or equal to the current amplitude at a constant potential. The current was increasing in both pulses $(+80 \mathrm{mV}$ and $-80 \mathrm{mV}$, Fig. 20). A different set of protocols were used for $\triangle$ PASCap (Fig. 21). In response to square pulse $(+80 \mathrm{mV}$ and $-80 \mathrm{mV}), \Delta$ PASCap did not show the increase of current amplitude (data not shown). We, therefore, replaced $-80 \mathrm{mV}$ with $-20 \mathrm{mV}$ (weak depolarizing potentials) and $+80 \mathrm{mV}$ with $+50 \mathrm{mV}$ (moderate depolarizing potentials).

The moderate potential range $(+50 \mathrm{mV})$ represents the range where $\triangle \mathrm{PASCap}$ inactivation was observed. $\triangle$ PASCap showed larger current amplitude at $+50 \mathrm{mV}$ in comparison to a constant potential. A square pulse protocol $(-20 \mathrm{mV}$ and $+80 \mathrm{mV})$ was then applied to alternate between weak and strong potentials. The increase in current amplitude was not detected. We also alternated the voltage between $-20 \mathrm{mV}$ and $0 \mathrm{mV}$ (another set of moderate potentials); however, no increase in current amplitude was measured. Therefore, a very narrow range of potentials could show this increase. In comparison to E600R and $\Delta \mathrm{eag}$, the increase observed was smaller in amplitude.

We also tested WT with the square pulses (Fig. 22). HEK cells were stably transfected with $\mathrm{K}_{\mathrm{v}} 10.1$ and recorded using whole-cell patch-clamp. Voltage was alternated between $+80 \mathrm{mV}$ and $-80 \mathrm{mV}$ with a frequency of $100 \mathrm{~Hz}$ for $600 \mathrm{~ms}$. The current amplitude decreased with square pulses. During the square pulses the current 
increased at $+80 \mathrm{mV}$, while it decreased at $-80 \mathrm{mV}$ (Fig 22C). The WT is expected to have limited access to $\mathrm{O}_{1}$, which could explain why we see less current when we alternated the voltage.

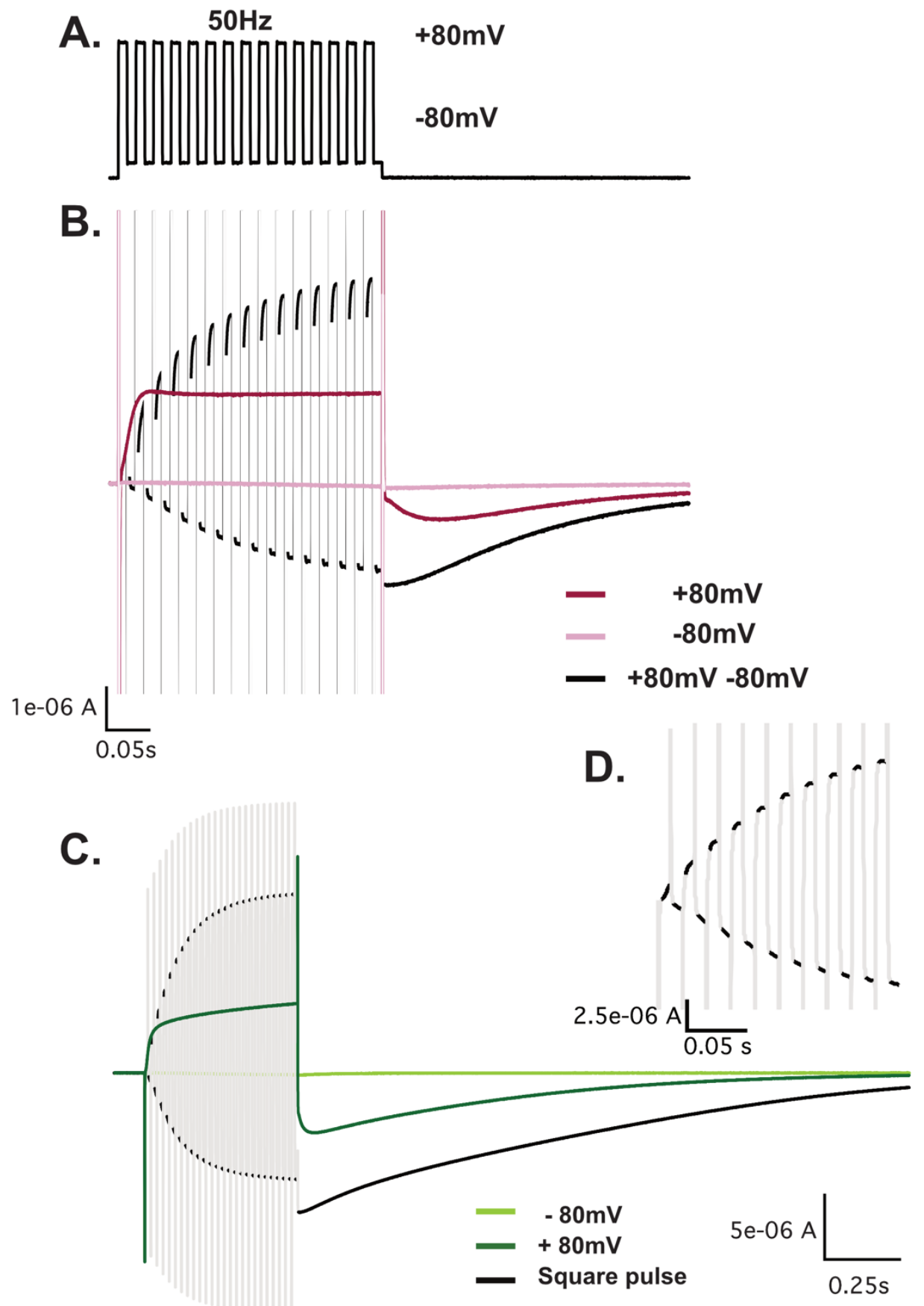

Figure 19. Alternating the voltage between weak and strong depolarizations recover E600R and $\Delta$ eag current at strong potentials. A) Voltage protocol (Square pulses, $300 \mathrm{~ms}$ E600R, 500ms $\Delta$ eag); Recordings were performed in $60 \mathrm{mM} \mathrm{K}^{+}$solution without leak subtraction. Equivalent constant voltage steps $(+80,-80 \mathrm{mV})$ were applied. B) E600R representative trace $(\mathrm{N}=7)$. Capacitance was faded to visualize the response. C) $\Delta$ eag representative trace $(\mathrm{N}=5)$. Capacitance was faded to visualize the response. D) Enlarged view of the $\Delta$ eag current response to square pulse. 

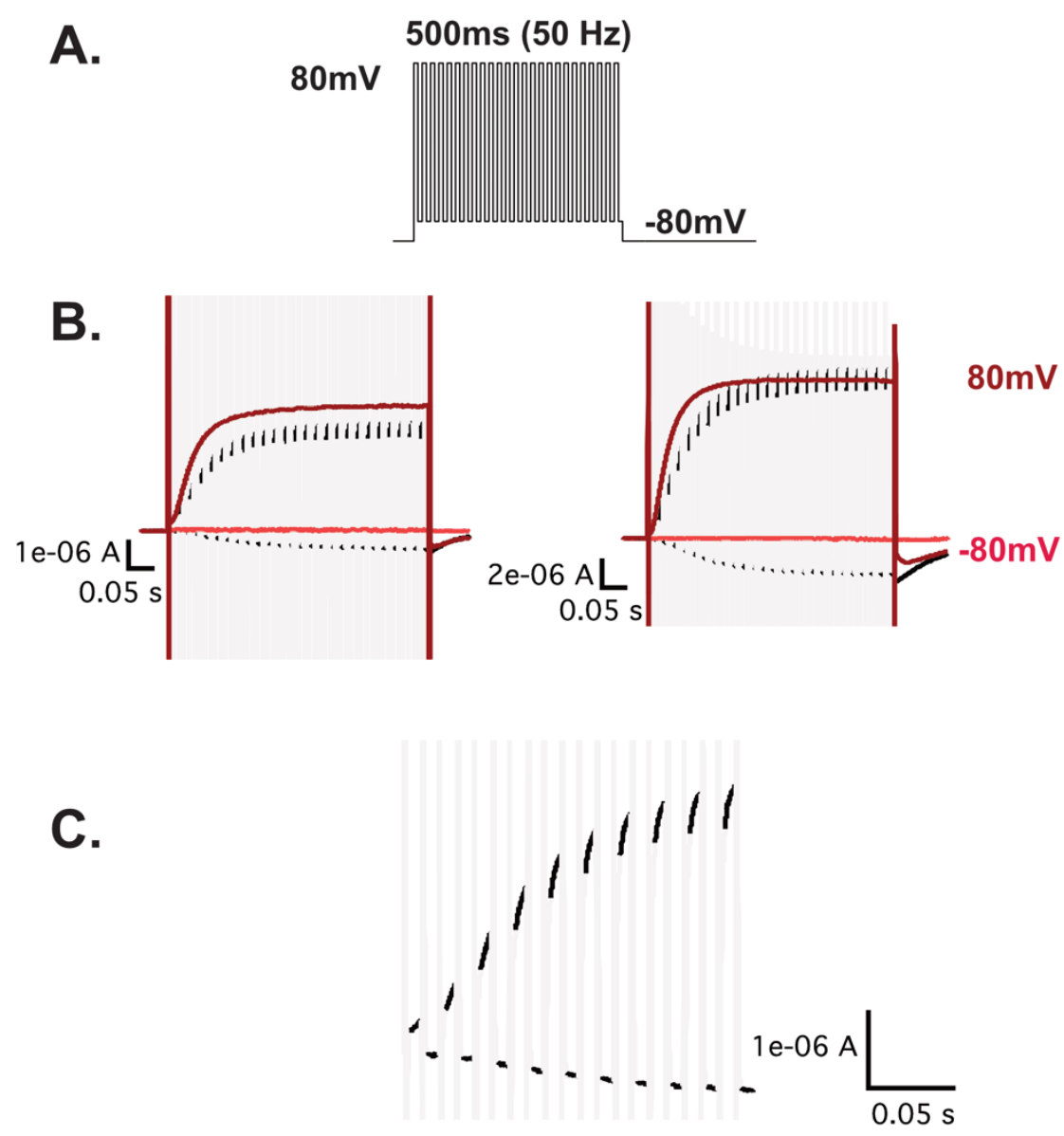

Figure 20. No remarkable difference in $\Delta 2-10$ current amplitude when the voltage was alternated between +80 and $\mathbf{- 8 0} \mathbf{m V}$. A.) Alternating voltage protocols were recorded in $60 \mathrm{mM} \mathrm{K}^{+}$solution. Equivalent constant voltage steps were also used. Leak subtraction was not performed. B.) Representative current traces (Left, $\mathrm{N}=3$; Right, $\mathrm{N}=2$ ). Capacitance was faded to visualize the response. $\mathbf{C}$.) Enlarged view of the current response. 


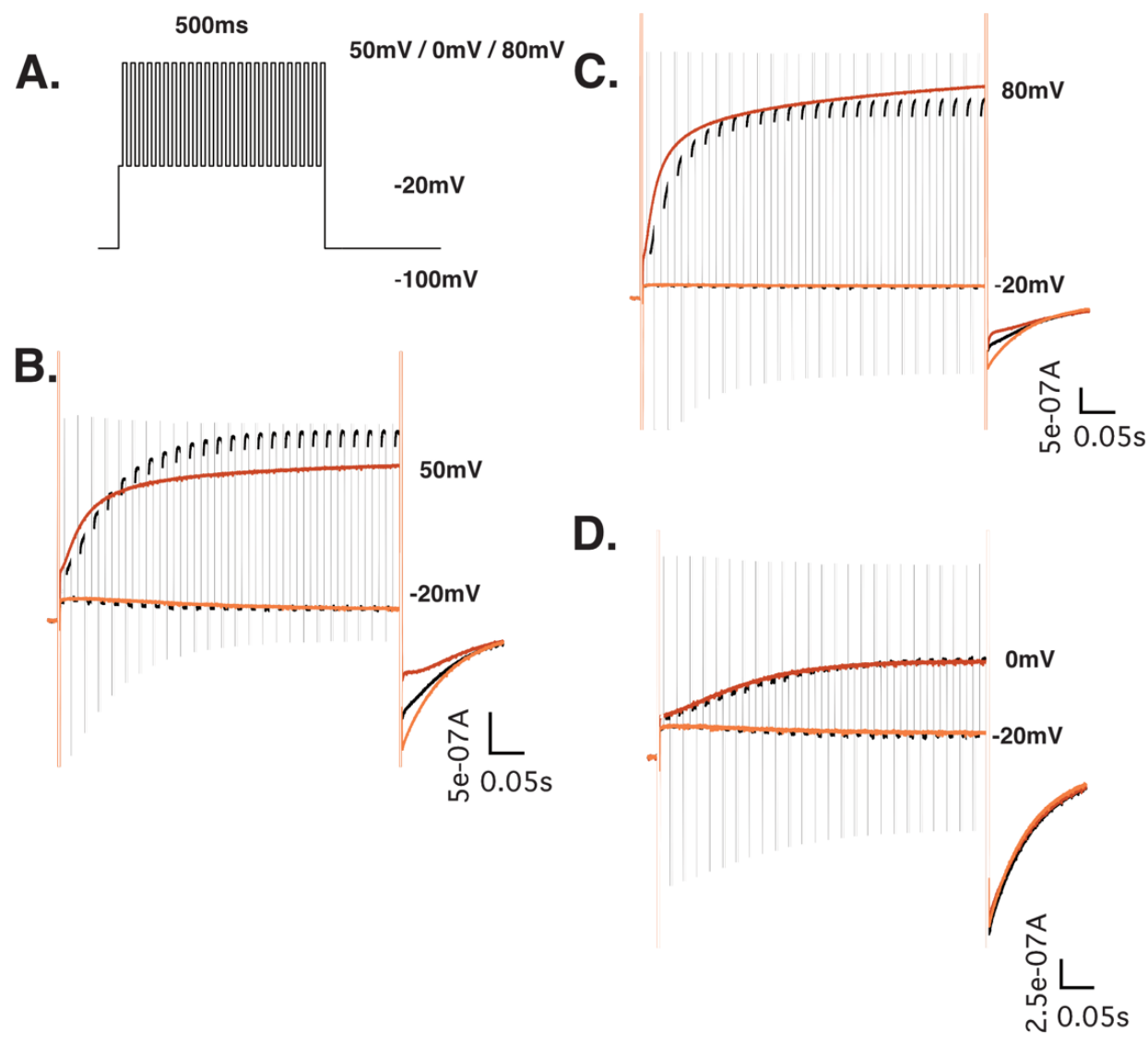

Figure 21.Alternating the voltage between -20 and $50 \mathrm{mV}$ recovers apparent inactivation. A) Voltage protocol (Square pulse,50Hz) used. Equivalent constant voltage steps were applied. Leak subtraction was avoided. Recording was performed in $60 \mathrm{mM} \mathrm{K}^{+}$solution. Representative recordings $(\mathrm{N}=6)$ of alternating the voltage between: B) -20 and $50 \mathrm{mV} \mathrm{C)} \mathrm{-20} \mathrm{and} 80 \mathrm{mV}$ E) -20 and $0 \mathrm{mV}$ Capacitance was faded to visualize the response. 

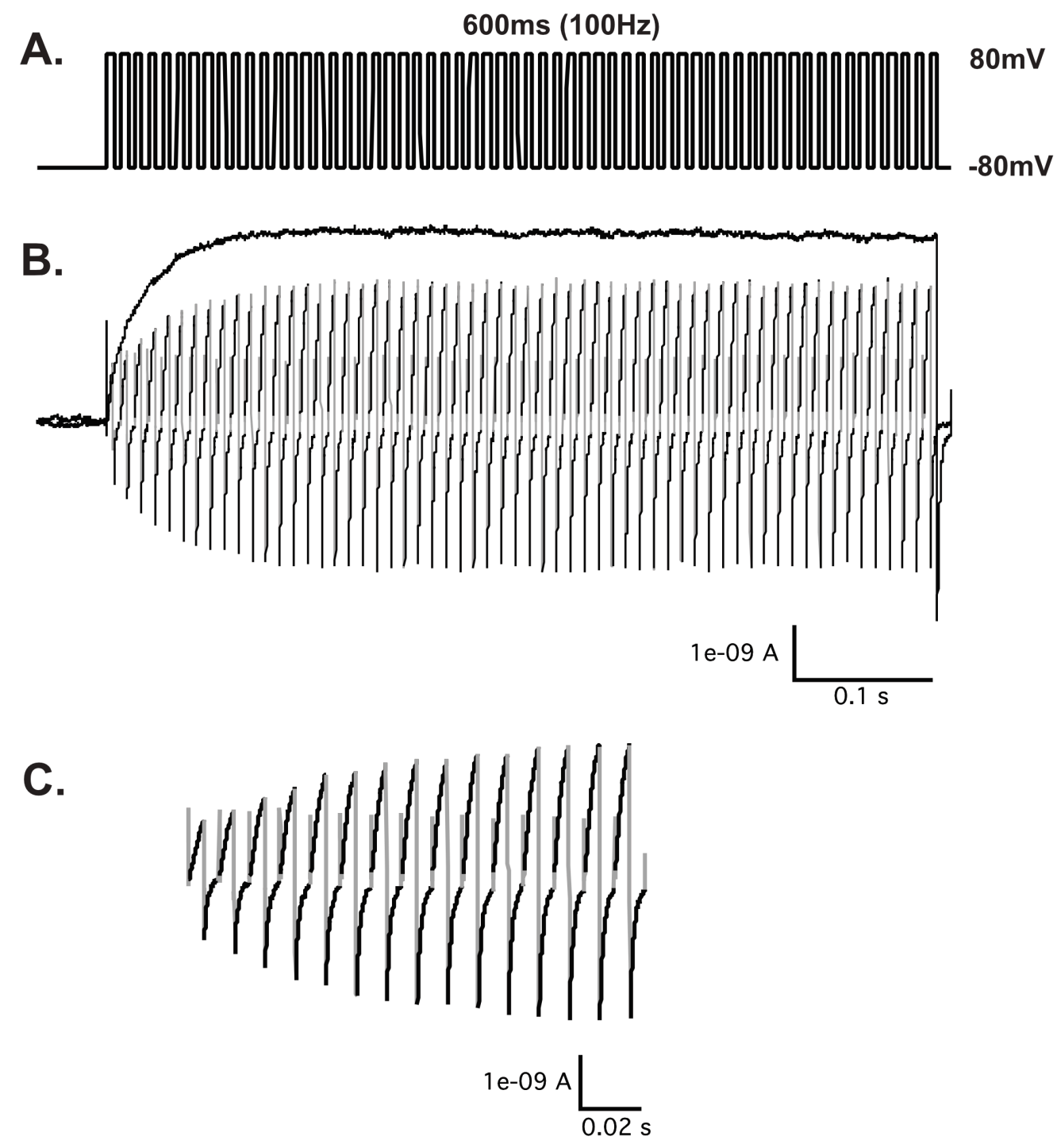

Figure 22.WT response to alternating the voltage between weak and strong depolarizations. A) Voltage protocol (Square pulses); Recordings were performed in $60 \mathrm{mM} \mathrm{K}^{+}$solution with leak subtraction. Equivalent constant voltage steps $(+80$, $80 \mathrm{mV}$ ) were applied. B) Current response. Capacitance was faded to visualize the response. C) Enlarged view of the current response to the square pulse

We hypothesized that $\mathrm{O}_{1}$, which dominates at weak and moderate potentials, has higher conductance than $\mathrm{O}_{2}$. To further test this hypothesis, we designed I-V protocols with pre-pulse potentials: a) A pre-pulse of weak depolarization B) A prepulse of strong depolarization. We focused on E600R and $\Delta$ eag, the two mutants that showed an increase in current amplitude in response to the square pulses (see above). 
Oocytes injected with E600R were subjected to $20 \mathrm{mV}$ depolarizing steps from $160 \mathrm{mV}$ to $+120 \mathrm{mV}$ for $300 \mathrm{~ms}$. The voltage steps were preceded by a 300 -ms pre-pulse a) $-20 \mathrm{mV}$ (weak depolarization) b) $+60 \mathrm{mV}$ (strong depolarization) (Fig. 23). Oocytes were held at $-100 \mathrm{mV}$, and leak subtraction was not performed. As a control, we used E600R GV recording (see above; Fig. 23A). In these recordings, the channels activated from $-100 \mathrm{mV}$ (holding potential). The activation at strong potentials (starting $+80 \mathrm{mV}$ ) showed an initial peak: a fast-activating component that inactivated rapidly. A strong depolarizing pre-pulse $(+60 \mathrm{mV})$ showed no initial peak at moderate and strong potentials. The absence of the peak could be attributed to the inactivation of this component at $+60 \mathrm{mV}$. However, a weak depolarizing pre-pulse $(-20 \mathrm{mV})$ induced an initial peak current at moderate and strong potentials $(+20 \mathrm{mV}$ to $+120 \mathrm{mV})$. The peak varied in amplitude among oocytes but was consistently observed. We compared the peak obtained with $-20 \mathrm{mV}$ pre-pulse to the peak observed with the GV of the same oocyte. The current response at $+60 \mathrm{mV}$ was normalized, and traces were superimposed (Fig. 23D). A pre-pulse of $-20 \mathrm{mV}$ showed larger initial peak. The peak indicates that a state of higher conductance dominates at $-20 \mathrm{mV}$.

$\Delta$ eag was subjected to a similar protocol, albeit with longer pre-pulse and test pulse duration (1s each). A pre-pulse of $-20 \mathrm{mV}$ was used to represent weak depolarizations, while a strong pre-pulse of $+80 \mathrm{mV}$ was used. An initial peak current was observed in response to $-20 \mathrm{mV}$, but not with a pre-pulse of $+80 \mathrm{mV}$ and $-100 \mathrm{mV}$ (Fig. 23E). To visualize the initial peak, we superimposed current traces at $+80 \mathrm{mV}$ with a pre-pulse of $-20 \mathrm{mV}$ and $-100 \mathrm{mV}$ (Fig. 23F). We attribute this peak to a difference in conductance between $-20 \mathrm{mV}$ and $+80 \mathrm{mV} ;-20 \mathrm{mV}\left(\mathrm{O}_{1}\right)$ favors a state of higher conductance than the state occupied at $+80 \mathrm{mV}\left(\mathrm{O}_{2}\right)$. In summary, pre-pulse protocols support our interpretations of square pulses: $\mathrm{O}_{1}$ appears to have higher conductance than $\mathrm{O}_{2}$. 


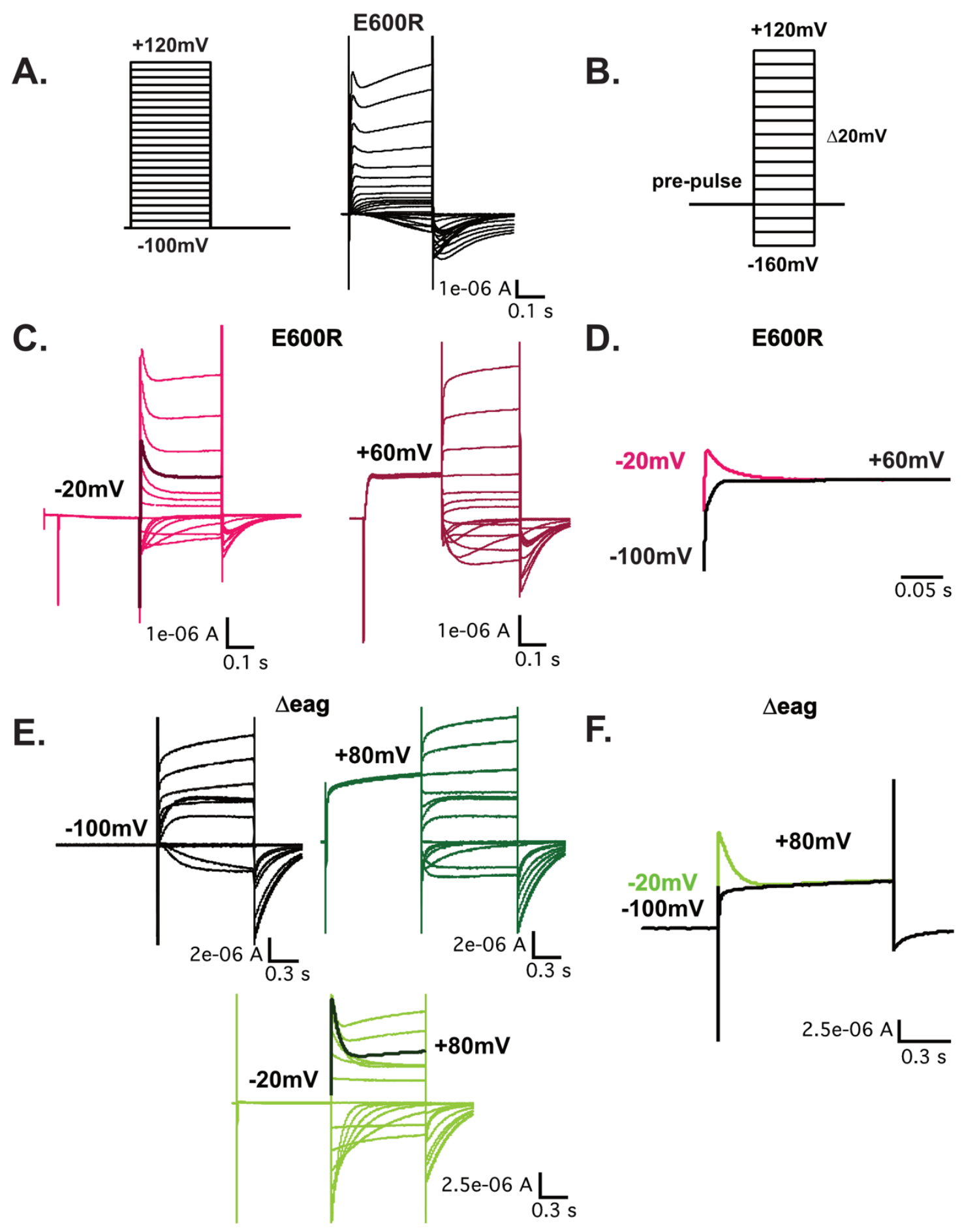

Figure 23. Weak depolarizations show higher conductance than strong potentials. Recordings were performed in $60 \mathrm{mM} \mathrm{K}^{+}$solution. A) Voltage protocol (left) and E600R current response (right). B) Voltage stimulus applied C) E600R Current response to protocol in B: pre-pulse of -20 and $+60 \mathrm{mV}$. $300 \mathrm{~ms}$ pulses and prepulses. D) Normalized current response to $+60 \mathrm{mV}$. Pre-pulse of $-20 \mathrm{mV}$ is compared to $-100 \mathrm{mV}$ (holding potential) E) $\Delta$ eag current response: pre-pulse of $-100,+80$ and $20 \mathrm{mV}$. $1 \mathrm{~s}$ pulses and pre-pulses. F) Overlay current response $(+80 \mathrm{mV})$, pre-pulse -20 and $-100 \mathrm{mV}$. 


\subsubsection{The first component of the biphasic GV represents a common gating step across mutants}

The biphasic GV of the mutants ( $\Delta 2-10, \Delta \mathrm{PASCap}, \Delta \mathrm{eag}$, and E600R) led us to hypothesize that two-voltage dependent gating steps occur in the mutants. Each step would have a sigmoidal GV curve at different range of potentials. The first step would occur at hyperpolarized potentials, while the second step would require stronger depolarizations. Therefore, two sigmoidal GV curves can be observed at separate voltage ranges. The first step would represent access to the first open state $0_{1}$, while the second step would grant access to a second open state $\mathrm{O}_{2}$. As shown above, $\mathrm{O}_{1}$ may have a higher conductance than $\mathrm{O}_{2}$ (see chapter 3.2.4). Therefore, the transition from a higher conducting state $\left(\mathrm{O}_{1}\right)$ to a lower conducting state $\left(\mathrm{O}_{2}\right)$ could explain the rectifying behavior (the transition step between the two GVs). It is important to note that the terms "early" and "late" do not indicate the sequence of the gating events, rather the voltage range where they appear.

The fitting equation was formulated to represent each component: 1) GV for $\mathrm{O}_{1}$ $\left.\left(A_{1}, V h_{1}, K_{1}\right) 2\right) G V$ for $\left.O_{2}\left(A_{2}, V h_{2}, K_{2}\right) 3\right)$ voltage-dependent transition $\left(V_{3}, K_{3}\right)$. The fitting was performed in collaboration with Dr. Andreas Neef.

$$
\begin{gathered}
f(V)=\mathrm{A} 0+\left(\frac{\mathrm{A} 1}{1+\mathrm{e}^{\frac{\mathrm{Vh} 1-\mathrm{V}}{\mathrm{K} 1}}}\right) *\left(1-\left(\frac{1}{1+\mathrm{e}^{\frac{\mathrm{Vh} 3-\mathrm{V}}{\mathrm{K} 3}}}\right)\right)+ \\
\left(\frac{\mathrm{A} 2}{1+\mathrm{e}^{\frac{\mathrm{Vh} 2-\mathrm{V}}{\mathrm{K} 2}}}\right) *\left(\frac{1}{1+\mathrm{e}^{\frac{\mathrm{Vh} 3-\mathrm{V}}{\mathrm{K} 3}}}\right)
\end{gathered}
$$

\section{Equation 6}

The first component was similar across mutants, suggesting that the first gating transition is common in the four mutants. Therefore, a global fit was performed using Eq. 5, and the first component $\left(\mathrm{A}_{1}, \mathrm{Vh}_{1}\right.$, and $\left.\mathrm{K}_{1}\right)$ was linked across mutants. The result 
of the fit is shown in Fig. 24 and Table 9. The GV for $\mathrm{O}_{1}$ is colored purple and the transition step in black, while the GV for $\mathrm{O}_{2}$ was color-coded with the respective mutant. The sigmoidal curve for $\mathrm{O}_{1}$ appeared at hyperpolarized potentials with a $\mathrm{Vh}=$ $-38 \mathrm{mV}$. The sigmoidal curve for $\mathrm{O}_{2}$ was shifted to the right; $\Delta 2-10$ was the least shifted $(\mathrm{Vh} 2=0.037 \mathrm{~V})$, while $\Delta$ PASCap $(\mathrm{Vh} 2=0.07 \mathrm{~V}), \Delta$ eag $(\mathrm{Vh} 2=0.09 \mathrm{~V})$, and E600R $(\mathrm{Vh} 2=0.099 \mathrm{~V})$, showed a larger shift. $\Delta$ eag showed the smallest total conductance $(\mathrm{A} 2=$ $0.144 \mathrm{v}$ ) and the most shifted transition $(\mathrm{Vh}=0.042 \mathrm{~V})$. The shift in voltage dependence transition to the right and the small amplitude could explain its distinct GV; the second phase is not as prominent, and the transition occurs at later potentials. In summary, the fitting validates our hypothesis; the first step is common among the four mutants.

We hypothesize that the first step relies on the sensor's movement, while the second step depends on a (voltage-dependent) movement of the cytoplasmic ring. Therefore, different deletions in the ring component differ in the second component and the transition phase, while the first component could be linked.

Table 9.Parameters of a global fit that linked the first component of the biphasic response

\begin{tabular}{|l|l|l|l|l|}
\hline & $\Delta 2-10$ & $\Delta$ PASCap & $\Delta$ eag & E600R \\
\hline $\mathrm{A}_{0}$ & -0.0212383 & -0.0212383 & -0.0212383 & -0.0212383 \\
\hline $\mathrm{A}_{1}$ & 1.15173 & 1.15173 & 1.15173 & 1.15173 \\
\hline $\mathrm{Vh}_{1}$ & -0.0381095 & -0.0381095 & -0.0381095 & -0.0381095 \\
\hline $\mathrm{K}_{1}$ & 0.0139869 & 0.0139869 & 0.0139869 & 0.0139869 \\
\hline $\mathrm{A}_{2}$ & 1.09311 & 1.17301 & 0.144657 & 1.68244 \\
\hline $\mathrm{Vh}_{2}$ & 0.0378328 & 0.0720457 & 0.0900246 & 0.099627 \\
\hline $\mathrm{K}_{2}$ & 0.0326141 & 0.0261325 & 0.00953164 & 0.0491352 \\
\hline $\mathrm{Vh}_{3}$ & 0.00540997 & -0.0364061 & 0.0421699 & -0.00852711 \\
\hline $\mathrm{K}_{3}$ & 0.0328496 & 0.0364433 & 0.0154282 & 0.016332 \\
\hline
\end{tabular}


A.
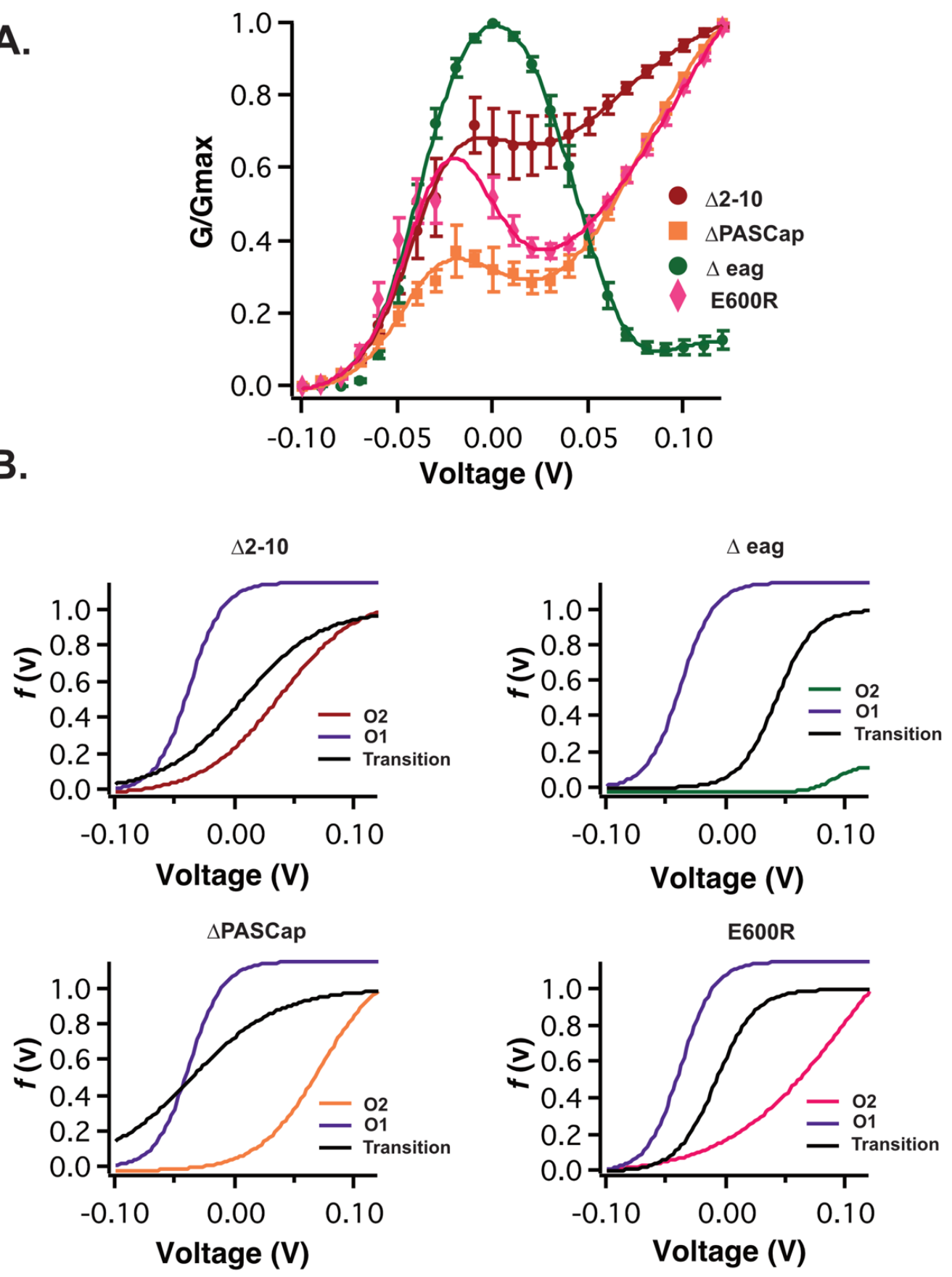

Figure 24. Individual components of a two-sigmoidal fit of the biphasic GV. A) Global fit of the mutants: $\Delta 2-10, \triangle$ PASCap, $\Delta$ eag and E600R using LevenbergMarquardt algorithm. The first component was linked across mutants. The fit was performed using Eq. 5. B) Individual components representing $\mathrm{O}_{1}$ (first component) and $\mathrm{O}_{2}$ (second component), and the transition in between. 


\subsection{6 $\Delta$ eag retains the biphasic behavior in the presence of $\mathrm{Mg}^{2+}$}

Since we hypothesized that the first component is correlated to the movement of the voltage sensor, and $\mathrm{Mg}^{2+}$ is known to slow down the S4 movement (Terlau et al., 1996). Therefore, we added $\mathrm{Mg}^{2+}$ to the external solution and examined its effect on the biphasic behavior (Fig. 25). We used $1 \mathrm{mM}$ and $5 \mathrm{mM} \mathrm{Mg}^{2+}$ in NFR solution. Then, we applied voltage stimuli from -100 to $+120 \mathrm{mV}$ for $300 \mathrm{~ms}$ at $20 \mathrm{mV}$ steps and measured the average current at the end of the depolarizing pulse (end-pulse current), and then normalized it to the maximum (I/Imax). The characteristic biphasic IV was observed in NFR solution. Increasing the $\mathrm{Mg}^{2+}$ concentration seemed to decrease the first component. This observation could be misleading since this is a slow component, and it might require more time to develop. Therefore, we repeated the protocol with a longer duration (1s) and could then observe the two components in the presence or absence of $\mathrm{Mg}^{2+}$. Increasing the $\mathrm{Mg}^{2+}$ concentration apparently shifted the first component of the I-V curve to the right., but this could be just an effect of the increased duration needed. 
A.

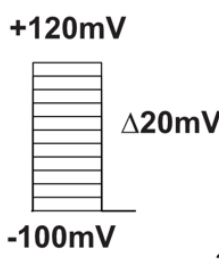

$$
\text { 2.5e-06A } \frac{\mathrm{L}}{0.1 \mathrm{~s}}
$$

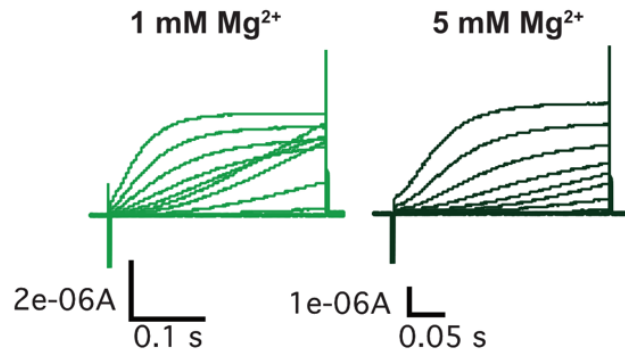

B.

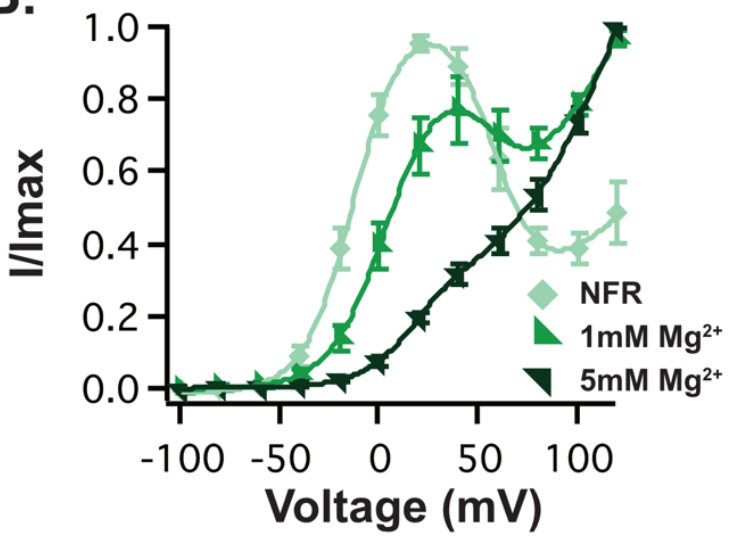

C.

NFR $1 \mathrm{~s}$
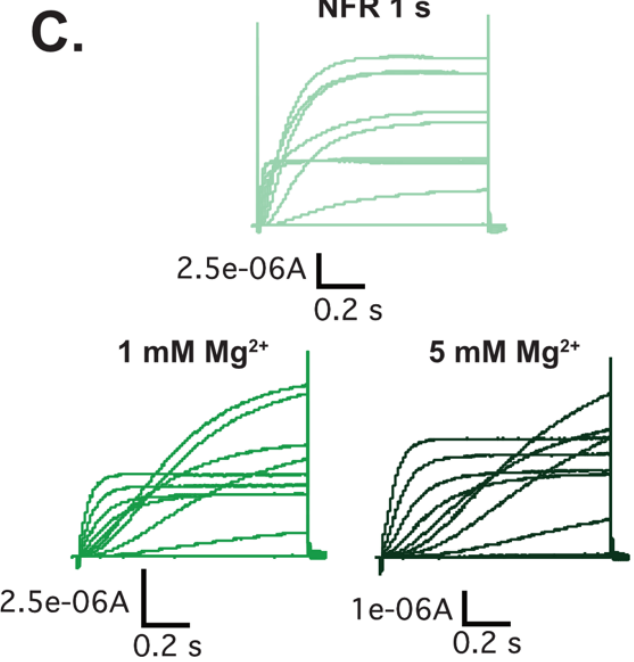

D.

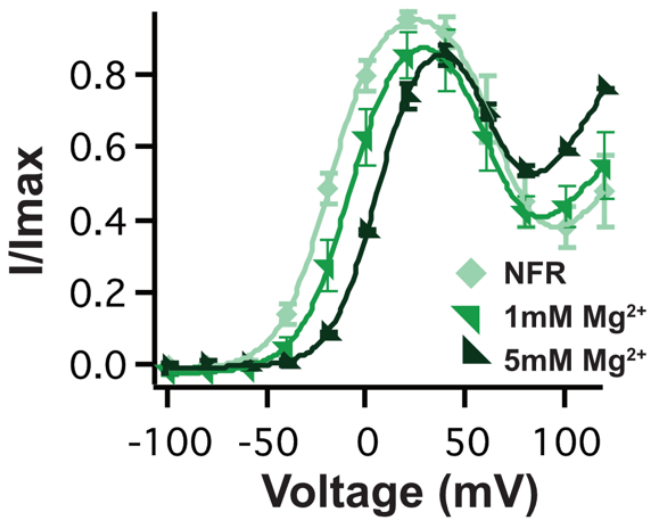

Figure 25. $\Delta$ eag I-V retains the biphasic behavior in the presence of $\mathrm{Mg}^{2+}$ but the curve is shifted to the right. A) Voltage protocol applied (upper left panel). Representative traces for $300 \mathrm{~ms}$ B) Normalized end-pulse current (300ms) was plotted against voltage. C) Representative traces for 1s D) Normalized end-pulse current (1s) was plotted against voltage. 


\subsection{7 $\Delta 2$-10 loses the biphasic GV upon disrupting a covalent connection between S4 and S5 (L341.split)}

To further test our prediction that the movement of the sensor underlies the first gating step, we interrupted the covalent connection after L341 residue of $\Delta 2-10$ mutant: $\Delta 2-10$. L341 split.

The split channels were designed as separate pore and voltage-sensing domains. cRNA of both domains were injected into Xenopus laevis oocytes. The two domains assembled then within the oocytes as functional channels that lack a covalent connection (Lorinczi et al., 2016). We used a voltage-sensing domain that ends at residue L341 and lacks the first ten residues (N-L341.D2-10) and co-expressed it with a pore domain that starts at D342 and spans to the end of the C-terminus. We, then performed TEVC recordings (Fig. 26). We applied 10mV steps from -100 to $+120 \mathrm{mV}$. Leak subtraction $(\mathrm{P} / \mathrm{n})$ protocol was applied. Oocytes were perfused with $60 \mathrm{mM} \mathrm{K}^{+}$ solution. The tail current was used to calculate conductance (Eq. 2 and Eq. 3). Conductance was normalized to the maximum and plotted against voltage. The first component of characteristic rectifying GV observed with $\Delta 2-10$ was significantly attenuated. $\Delta 2$-10. L341 split showed remanent of the biphasic behavior. However, the first component was very small and shifted to more depolarized potentials. We then applied long depolarizing pulses (2s) to check for possible inactivation. $\Delta 2-10$. L341 split lost the apparent inactivation that was observed with $\Delta 2-10$. 


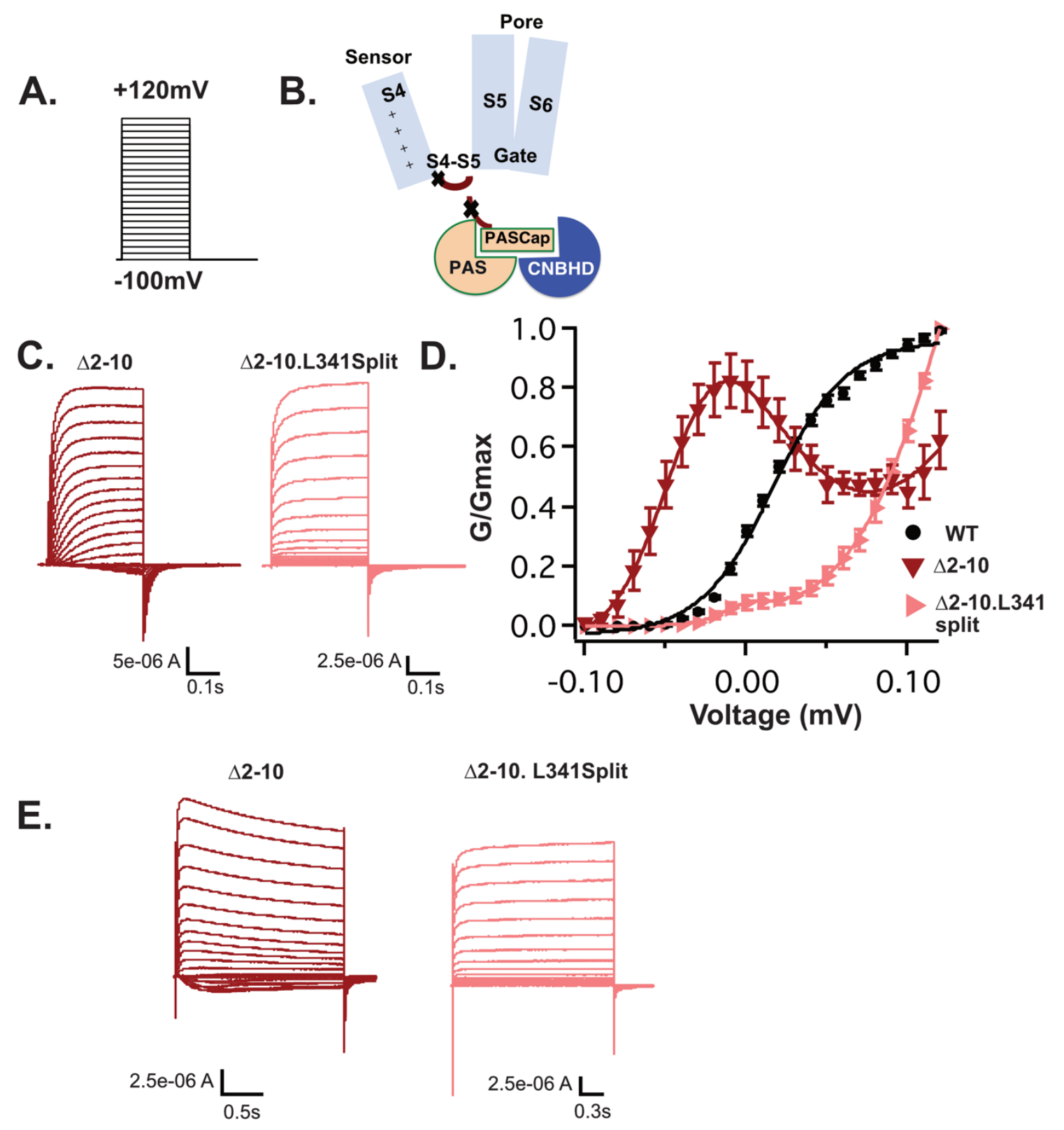

Figure 26. $\Delta 2$-10. L341split loses the first component of the biphasic GV. A) Voltage stimulus applied; $\Delta 10 \mathrm{mV}$ increasing order, $-160 \mathrm{mV}$ repolarizing $(\Delta 2-10)$. B) Mutant Cartoon C) Representative traces (300ms) D) Conductance-voltage relationship. Analysis using tail fitting Eq. 2 and Eq. 3. (WT N=9, $\Delta 2-10 \mathrm{~N}=10, \Delta 2-10$. L341 split N= 7) E) Representative traces (2s) 


\subsection{The first phase of the biphasic GV is stabilized with hyperpolarized pre-pulses and $\mathrm{Ca}^{2+}$-Calmodulin}

\subsubsection{Hyperpolarized pre-pulse potentiates the first phase of the biphasic IV}

$\mathrm{K}_{\mathrm{v}} 10.1$ channels transition through a series of closed states before reaching a conducting state. These complex transitions can be made visible by the change in activation kinetics after long hyperpolarizing pre-pulses preceding a test potential, commonly described as Cole-Moore shift. Hyperpolarization favors deep closed states; hence a sigmoidal activation is typically observed. In $\mathrm{K}_{\mathrm{v}} 10.1$, depolarized pre-pulse potential also accelerates activation kinetics (Hoshi and Armstrong, 2015; Ludwig et al., 1994). We examined the response of our mutants ( $\Delta 2-10, \Delta$ PASCap and E600R) to long hyperpolarizing pre-pulses to test if Cole-Moore was retained. We used a test pulse of $+40 \mathrm{mV}$ and hyperpolarizing pulses from $-160 \mathrm{mV}$ to $-40 \mathrm{mV}$ in $20 \mathrm{mV}$ steps. Recordings were performed in $\mathrm{Cl}^{-}$free NFR solution to avoid contamination with outward $\mathrm{Cl}^{-}$current. Risetime $80 \%$ was then plotted versus pre-pulse potential. $\triangle$ PASCap and E600R showed slower activation kinetics than the WT at hyperpolarizing potentials (rise time $80 \%$ at $-160 \mathrm{mV}: \mathrm{WT}=0.056 \pm 0.005 \mathrm{~s}, \Delta \mathrm{PASCap}=0.23 \pm 0.015 \mathrm{~s}$, $\mathrm{E} 600 \mathrm{R}=0.24 \pm 0.038 \mathrm{~s}$ ). $\Delta 2-10$ was slightly slower than WT (rise time $80 \%$ at $-160 \mathrm{mV}$ : $\mathrm{WT}=0.056 \pm 0.005 \mathrm{~s}, \Delta 2-10=0.066 \pm 0.004 \mathrm{~s}$ ). WT showed a decrease in rise time as the pre-pulse potential increased. We fitted the response with a sigmoidal fit (WT, $N=8$, $\mathrm{Vh}=-106.34 \mathrm{mV}$; Mean $\pm \mathrm{SEM})$. A sigmoidal response could be observed with $\Delta 2-10$ and $\triangle$ PASCap; $\Delta 2-10$ showed a slight shift to depolarized potentials, while $\triangle$ PASCap and WT were similar $(\Delta 2-10, \mathrm{~N}=7, \mathrm{Vh}=-91.35 \mathrm{mV} ; \Delta$ PASCap, $\mathrm{N}=11, \mathrm{Vh}=-100.57 \mathrm{mV})$. E600R also showed acceleration. The sigmoidal response could be shifted to the left, and hence no saturation was detected. (E600R, N=11, Vh=-146 mV). In our hands, the rise time of $\Delta 2-10, \triangle$ PASCap, and E600R decreased with more depolarized pre-pulse potential similar to the WT (Fig. 27). 


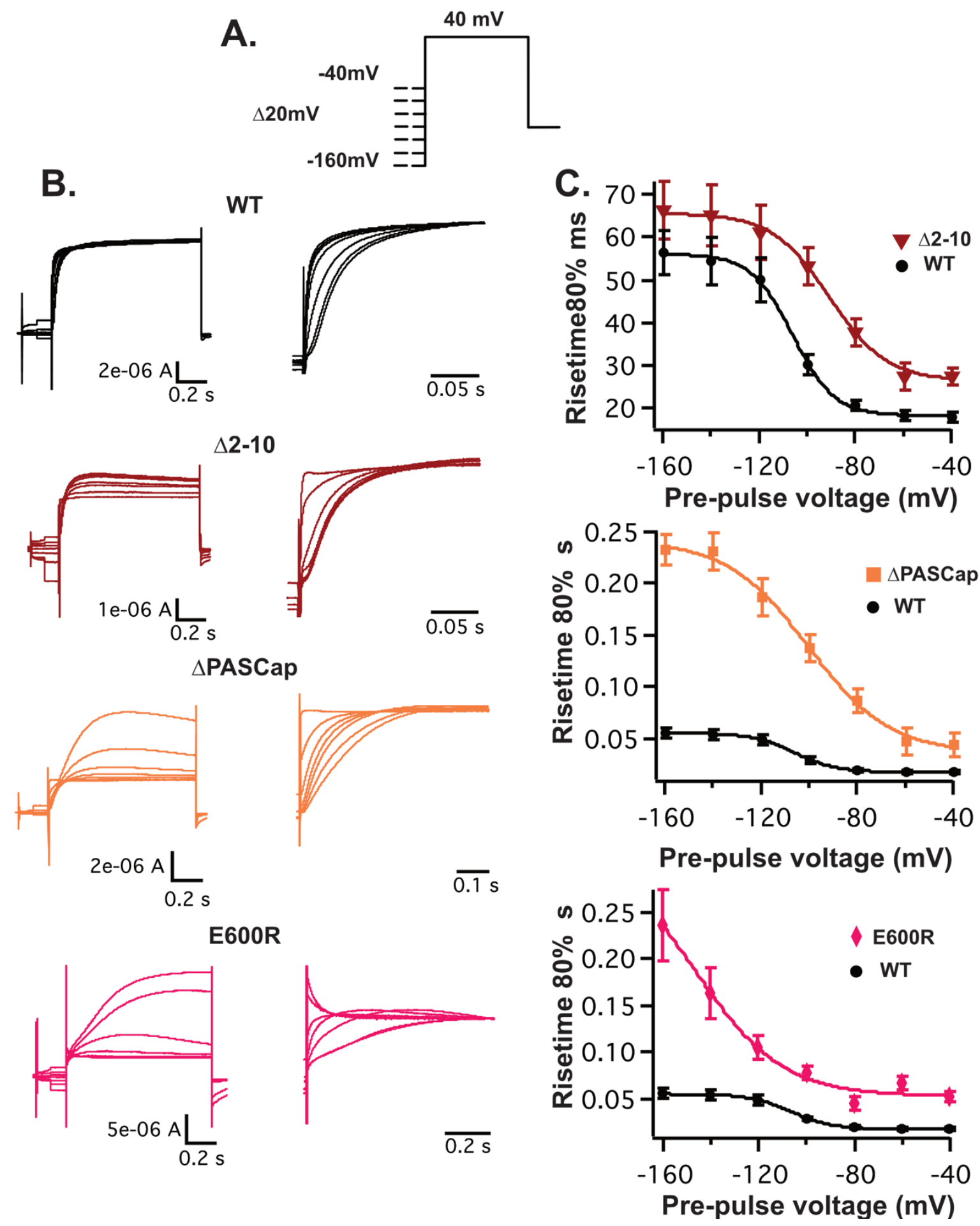

Figure 27.N-terminal deletions $(\Delta 2-10 ; \Delta$ PASCap) and E600R activation accelerates with depolarized pre-pulse potentials. A) Cole-Moore protocol; prepulse potential for $5 \mathrm{~s}$ (first and last $100 \mathrm{~ms}$ were recorded). Recording was performed in Cl- free NFR solution. Leak subtraction was not performed. B) Representative traces C) Time to reach $80 \%$ of maximum response (Rise time $80 \%$ ) was plotted vs. pre-pulse potential. Response was fitted with a sigmoid curve (WT, $\mathrm{N}=8, \mathrm{Vh}=-106.34 \mathrm{mV} ; \Delta 2-10$, $\mathrm{N}=7, \mathrm{Vh}=-91.35 \mathrm{mV} ; \Delta \mathrm{PASCap}, \mathrm{N}=11, \mathrm{Vh}=-100.57 \mathrm{mV}$; E600R, $\mathrm{N}=11, \mathrm{Vh}=-146 \mathrm{mV}$; mean \pm SEM) 
We observed a potentiation in the test current in response to hyperpolarized potential $\triangle$ PASCap and E600R response that caught our attention. We, therefore, plotted the normalized end-pulse current against the pre-pulse potential. The prepulse at $-20 \mathrm{mV}$ was used for normalization. In comparison to $-20 \mathrm{mV}$ pre-pulse, $-160 \mathrm{mV}$ pre-pulse potentiated $\triangle$ PASCap $(3.5 \mathrm{x} \pm 0.3)$ and E600R current $(3.98 \mathrm{x} \pm 0.85)$ at $+40 \mathrm{mV}$ (Fig. 28B, Fig. 29B) The test pulse $(+40 \mathrm{mV})$ belongs to a range of potentials where both current components $\left(\mathrm{O}_{1}\right.$ and $\left.\mathrm{O}_{2}\right)$ were detected. Therefore, we repeated the protocol with a test potential where only the second hypothetic component $\left(\mathrm{O}_{2}\right)$ would dominate $(+80 \mathrm{mV})$. The potentiation observed with $+40 \mathrm{mV}$ test pulse was lost when $+80 \mathrm{mV}$ was used (Fig. 28B and 29B). To confirm our observation, we recorded an IV protocol with a pre-pulse potential of $-160 \mathrm{mV}$ for $5 \mathrm{~s}$. Compared to a $-100 \mathrm{mV}$ pre-pulse, $-160 \mathrm{mV}$ potentiated $\triangle \mathrm{PASCap}$ and E600R current at moderate depolarization, which was mirrored by a change in the IV; the first component became more prominent (Figs. $28 \mathrm{~F}$ and 29F). The experiments were performed in $\mathrm{Cl}^{-}$free solutions to exclude contamination with outward $\mathrm{Cl}^{-}$currents, which could occur with hyperpolarized potentials. We concluded that hyperpolarization stabilizes $\mathrm{O}_{1}$, which is not favored at strong depolarization $(+80 \mathrm{mV})$. 

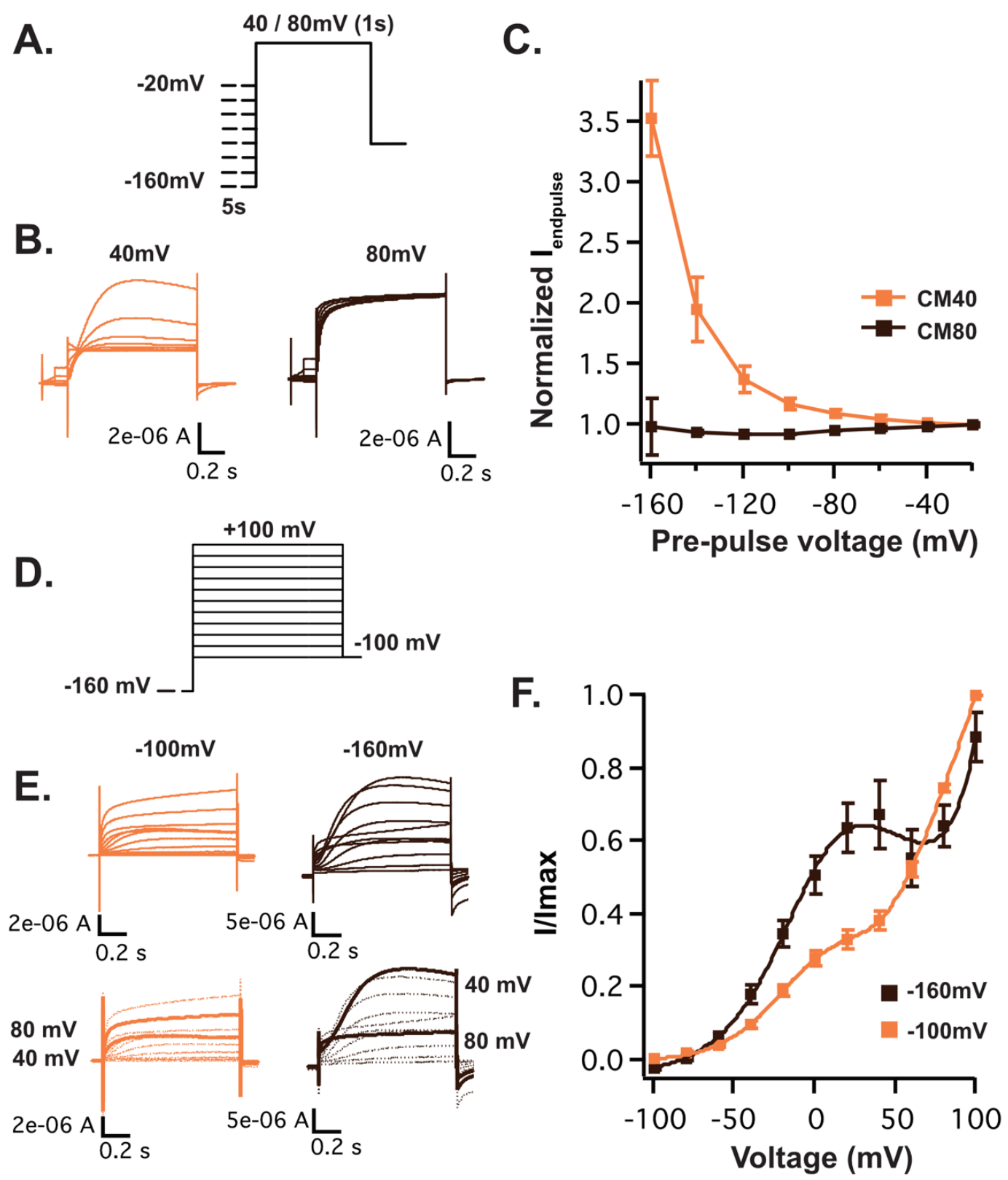

Figure 28. Hyperpolarizing pre-pulse potentiates $\triangle$ PASCap current at moderate potentials. A) Cole-Moore Protocol (CM): Pre-pulse for $5 \mathrm{~s}$ (only 200ms was recorded); $+40 \mathrm{mV}$ test pulse $(\mathrm{CM} 40)+80 \mathrm{mV}$ test pulse (CM80). No leak subtraction. Recording performed in $\mathrm{Cl}^{-}$free NFR solution. B) Representative traces: CM40 and CM80. C) Normalized end-pulse current (Iendpulse) vs. pre-pulse voltage. The response to a pre-pulse potential of $-20 \mathrm{mV}$ is used for normalization (CM40 N=8; CM80 N=6; \pm SEM) D) Voltage protocol applied; $5 \mathrm{~s}$ pre-pulse of $-160 \mathrm{mV}$ (only $200 \mathrm{~s}$ recorded) followed by a test pulses for $1 \mathrm{~s}$. Recording performed in $\mathrm{Cl}^{-}$free NFR solution. E) Representative traces. Lower traces highlights $40 \mathrm{mV}$ (moderate potentials; $\mathrm{O}_{1}$ ) and 80 $\mathrm{mV}$ (strong potential; $\mathrm{O}_{2}$ ) F) Normalized end pulse current (I/Imax) vs. test potential $( \pm$ SEM). The effect of $\mathbf{- 1 6 0} \mathbf{m V}(\mathrm{N}=8)$ compared to $-100 \mathrm{mV}(\mathrm{N}=8)$ pre-pulse. IV is fitted with a two-sigmoid (Eq. 5). (-160mV: $\mathrm{A}_{1}=6.16, \mathrm{Vh}_{1}=-2.6 \mathrm{mV}, \mathrm{K}_{1}=21.2, \mathrm{~A}_{2}=10.8$, $\mathrm{Vh}_{2}=148.6 \mathrm{mV}, \mathrm{K}_{2}=16.5$, =, $\mathrm{Vh}_{3}=-154.26 \mathrm{mV}, \mathrm{K}_{3}=98.75 ;-100 \mathrm{mV}: \mathrm{A}_{1}=0.39, \mathrm{Vh}_{1}=-19 \mathrm{mV}$, $\mathrm{K}_{1}=20, \mathrm{~A}_{2}=2.5, \mathrm{Vh}_{2}=118.5 \mathrm{mV}, \mathrm{K}_{2}=47, \mathrm{Vh}_{3}=41 \mathrm{mV}, \mathrm{K}_{3}=13.15$ ) 

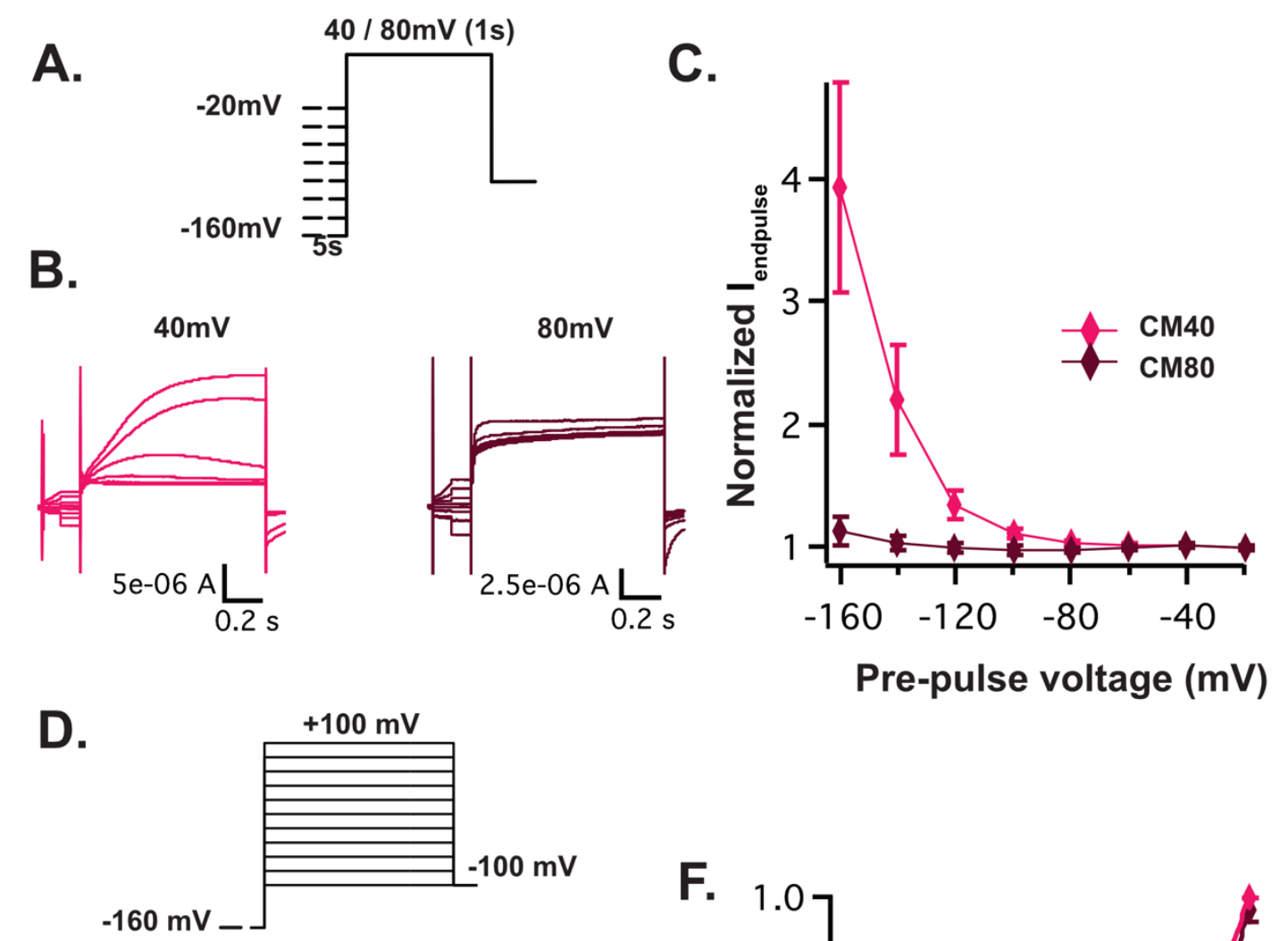

E.
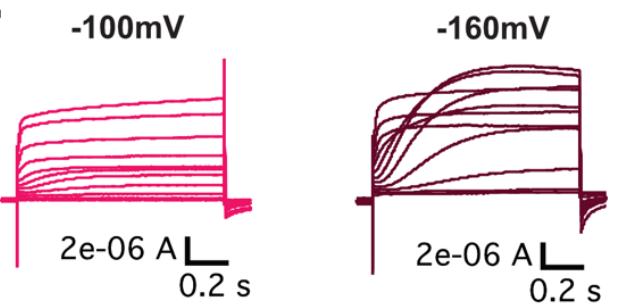

F.
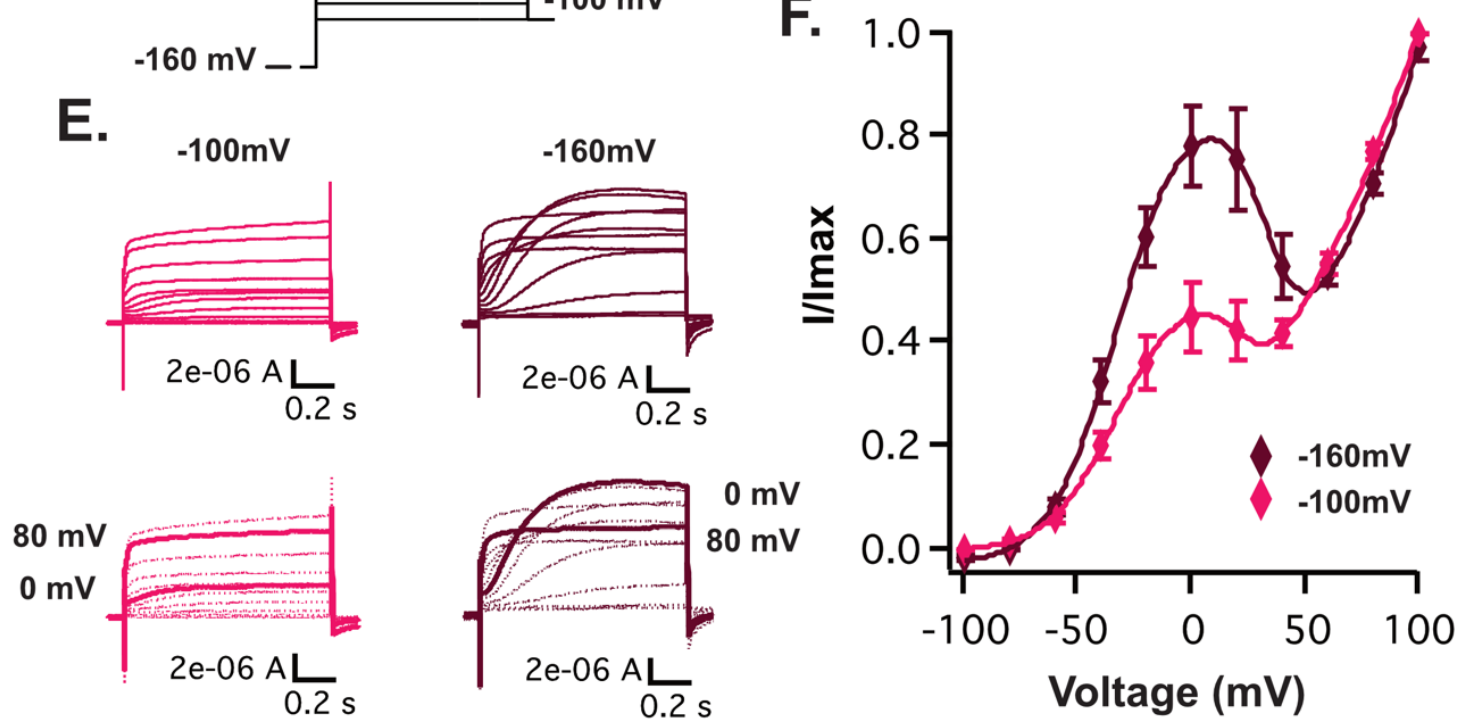

Figure 29.Hyperpolarizing pre-pulse potentiates E600R current at moderate potentials. A) Cole-Moore Protocol. Pre-pulse for $5 \mathrm{~s}$ (only $200 \mathrm{~ms}$ recorded); $+40 \mathrm{mV}$ test pulse (CM40), $+80 \mathrm{mV}$ testpulse (CM80). No leak subtraction. Recording performed in $\mathrm{Cl}^{-}$free NFR solution. B) Representative traces; CM40 and CM80. C) Normalized endpulse current (Iendpulse) is plotted vs. pre-puse voltage. The response to a pre-pulse potential of $-20 \mathrm{mV}$ is used for normalization. (CM40 N=11; CM80 N=12; \pm SEM) D) Voltage protocol applied; $5 \mathrm{~s}$ pre-pulse (only $200 \mathrm{~ms}$ recorded) of $-160 \mathrm{mV}$ followed by a test pulses of $1 \mathrm{~s}$. Recording performed in $\mathrm{Cl}^{-}$free NFR solution. E) Representative traces. Lower traces highlights $0 \mathrm{mV}$ (moderate potentials; $\mathrm{O}_{1}$ ) and $80 \mathrm{mV}$ (strong potential; $\mathrm{O}_{2}$ ) F) Normalized end pulse current (I/Imax) vs test potential ( \pm SEM). The effect of $-160 \mathrm{mV}(\mathrm{N}=6)$ compared to $-100 \mathrm{mV}(\mathrm{N}=6)$ pre-pulse IV is fitted with a twosigmoid Eq. 5. (-160mV: $A_{1}=0.9, \mathrm{Vh}_{1}=-33.3 \mathrm{mV}, \mathrm{K}_{1}=14.36, \mathrm{~A}_{2}=4.9, \mathrm{Vh}_{2}=173.77 \mathrm{mV}$, $\mathrm{K}_{2}=54.37, \mathrm{Vh}_{3}=35.47 \mathrm{mV}, \mathrm{K}_{3}=8.9 ;-\mathbf{- 1 0 0 m V}: \mathrm{A}_{1}=0.5, \mathrm{Vh}_{1}=-34 \mathrm{mV}, \mathrm{K}_{1}=14.37, \mathrm{~A}_{2}=3.4$, $\mathrm{Vh}_{2}=147 \mathrm{mV}, \mathrm{K}_{2}=53.6, \mathrm{Vh}_{3}=25.5 \mathrm{mV}, \mathrm{K}_{3}=8$ ) 


\subsection{2 $\mathrm{Ca}^{2+}-$ Calmodulin stabilizes the first phase of the biphasic IV}

$\mathrm{Ca}^{2+}$ Calmodulin inhibits WT current and potentiates $\triangle$ PASCap and E600R current at a single pulse potential $(+60 \mathrm{mV})$ (Lorinczi et al., 2016). To test the effect of $\mathrm{Ca}^{2+}$ Calmodulin on the biphasic IV of E600R and $\triangle$ PASCap, we designed a shortened IV protocol: we applied five voltage steps $(-80,-40,0,40,80 \mathrm{mV})$ with 20 s interval (Figs. $30 \mathrm{E}$ and 31E). The five steps were applied 200s after raising intracellular $\mathrm{Ca}^{2+}$ with ionomycin and thapsigargin $(5 \mu \mathrm{M})$ as reported by (Lorinczi et al., 2016). Ionomycin is a $\mathrm{Ca}^{2+}$ ionophore (Yoshida and Plant, 1992), while thapsigargin inhibits the ER $\mathrm{Ca}^{2+}$ ATPase and depletes the intracellular stores (Sehgal et al., 2017; Thurman et al., 2000). $\triangle$ PASCap response to a single pulse varied significantly during the first 100s (Lorinczi et al., 2016), we, therefore, applied our protocol $200 \mathrm{~s}$ after raising $\mathrm{Ca}^{2+}$ to avoid this variability. The protocol lasted for $100 \mathrm{~s}$ to abide by the $300 \mathrm{~s}$ previously reported to have a relatively constant rise of intracellular $\mathrm{Ca}^{2+}$. For both $\triangle \mathrm{PASCap}$ and E600R, $\mathrm{Ca}^{2+}$ rise induced potentiation of the current. This potentiation was not homogeneous across the voltage range tested and was more pronounced at $+40 \mathrm{mV}$. The overall effect was a general increase of current and that the increase in current with voltage lost its initial abrupt rise. Therefore, the IV was no longer biphasic (Fig. 30G, Fig. 31G). This would suggest that $\mathrm{Ca}^{2+}$-Calmodulin stabilizes $\mathrm{O}_{1}$ and prevents the transition to $\mathrm{O}_{2}$, which would result in a non-conducting channel in the wild type, and in a purely $\mathrm{O}_{1} \mathrm{IV}$ in the mutants.

In addition to this short IV, we also designed experiments with a ramp protocol $(-120 \mathrm{mV}$ to $+100 \mathrm{mV})$ for $5 \mathrm{~s}$. The ramp was repeated with $30 \mathrm{~s}$ intervals. After the application of ionomycin and thapsigargin, the response was recorded for $900 \mathrm{~s}$. Panel B in Figs. 30-32 shows the response after 60, 150, 300, 600, and 900s. The IV curve was normalized to the maximum response to visualize the change at the respective time points $(60,150,300,600,900$ s) (Panels D). $\triangle$ PASCap showed initial potentiation and an initial linear IV after $60 \mathrm{~s}(9.8 \mathrm{x} \pm 0.75 ; \mathrm{n}=4)$, followed by a decline that started already at 150 and $300 \mathrm{~s}(5.9 \mathrm{x} \pm 0.59 ; 4.77 \mathrm{x} \pm 0.58 ; \mathrm{n}=4)$ (Fig. 30B). The decline started earlier for strong depolarizations, while at moderate depolarizations -where $\mathrm{O}_{1}$ dominates- the response was sustained for a longer time. This pattern was conserved 
beyond the reported 300s, up to 900s. The IV changed from biphasic to linear after 60s of ionomycin and thapsigargin application (Fig. 30D). The IV retained a monophasic response within 600s; the IV became sigmoidal at 150s and showed rectification at strong potentials at 300s and 600s. The monophasic IV overlapped with the first component of the biphasic IV. E600R also showed an initial linear IV and potentiation (60s) (7.9x \pm 1.12 ; $\mathrm{n}=5$ ) (Fig. 31B). The response was sustained for longer than in $\triangle$ PASCap (150ms: $7.17 \mathrm{x} \pm 1 ; 300 \mathrm{~ms}$ : $7.6 \pm 1.16$; $\mathrm{n}=5$ ). However, the current declined in a similar pattern as $\triangle$ PASCap; the decline was first noticed at strong depolarization. The response of $\Delta 2-10$ to the ramp protocol was intriguing (Fig. 32). There was an initial inhibition of the current after 60 s, followed by a recovery at $150,300,600$, and $900 \mathrm{~s}$ (Fig. 32B). The IV retained the biphasic behavior. However, 150, 300, and 600s showed amplification of the first component of the IV compared to the control and 60s . We conclude that $\mathrm{Ca}^{2+} \mathrm{Calmodulin}$ could stabilize the first component of the IV, which we attribute to $0_{1}$. 

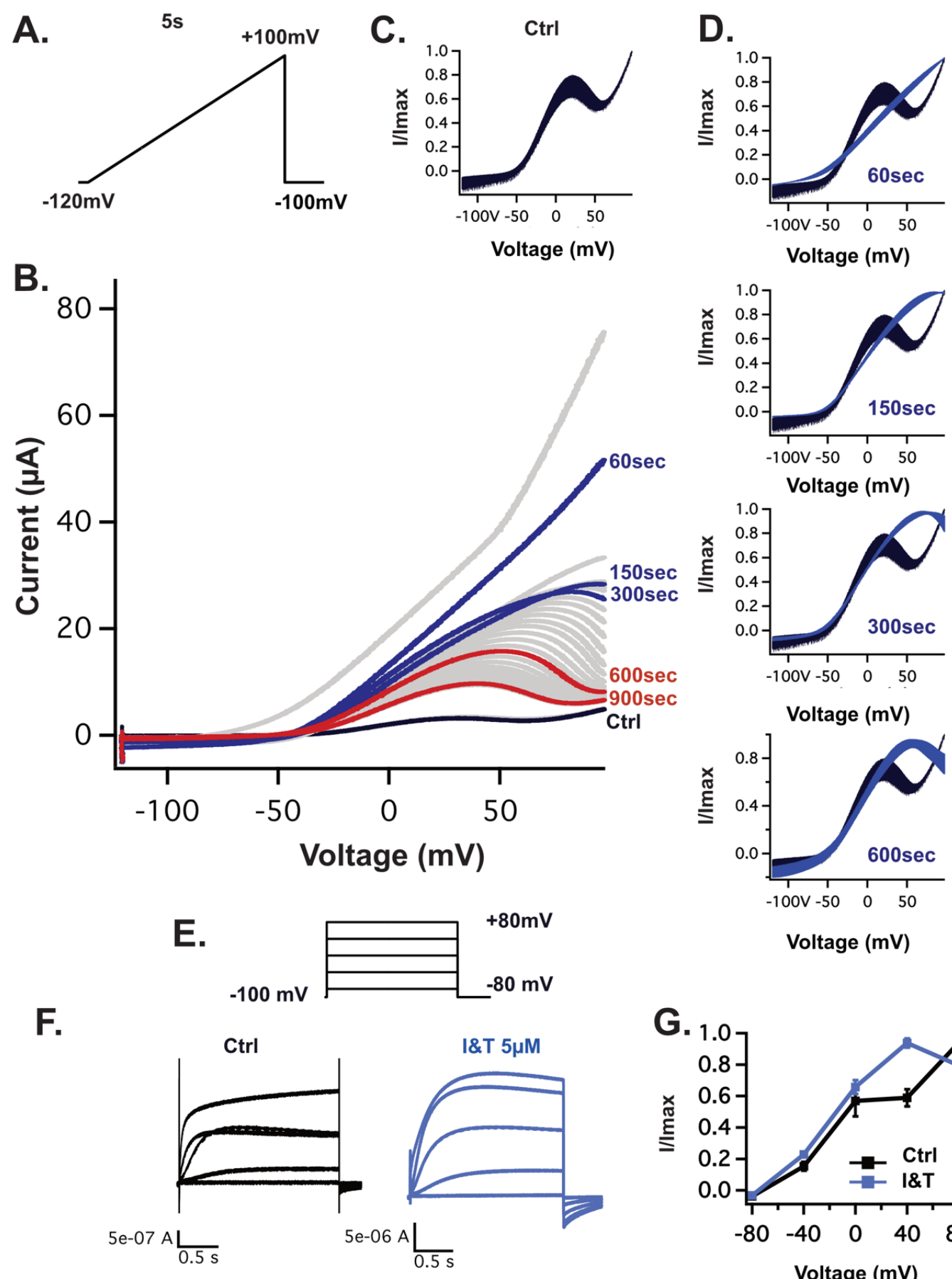

Voltage $(\mathrm{mV})$

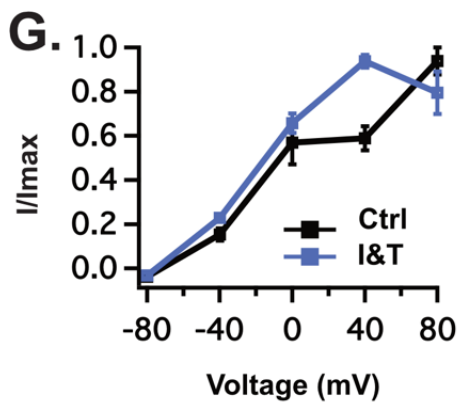

Figure 30. Ca ${ }^{2+-C a l m o d u l i n}$ potentiates $\triangle$ PASCap current converting the biphasic IV to a linear one. A) Ramp protocol(5s) repeatedly applied with a 30s interval. Leak subtraction wasn not performed B) Representative current response to the ramp protocol in A. $5 \mu \mathrm{M}$ ionomycin and thapsigargin (I\&T) were applied. Blue traces are within 300s while red traces exceed 300 s. Normalized current vs. voltage (IV) ( $n=5 ; \pm$ SEM) for C) Ctrl current (dark blue) D) Time points (60, 150 300,600 s) (lighter blue) E) Voltage steps applied after $200 \mathrm{~s}$ of applying $5 \mu \mathrm{M}$ I\&T; $2 \mathrm{~s}$ pulses from +80 to -80 (decreasing order) with $40 \mathrm{mV}$ step. F) Current response G) Normalized end pulse current vs. voltage (IV) $(\mathrm{N}=8 ; \pm \mathrm{SEM})$. 

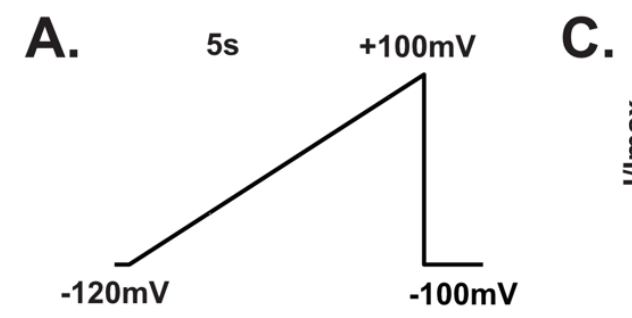

B.
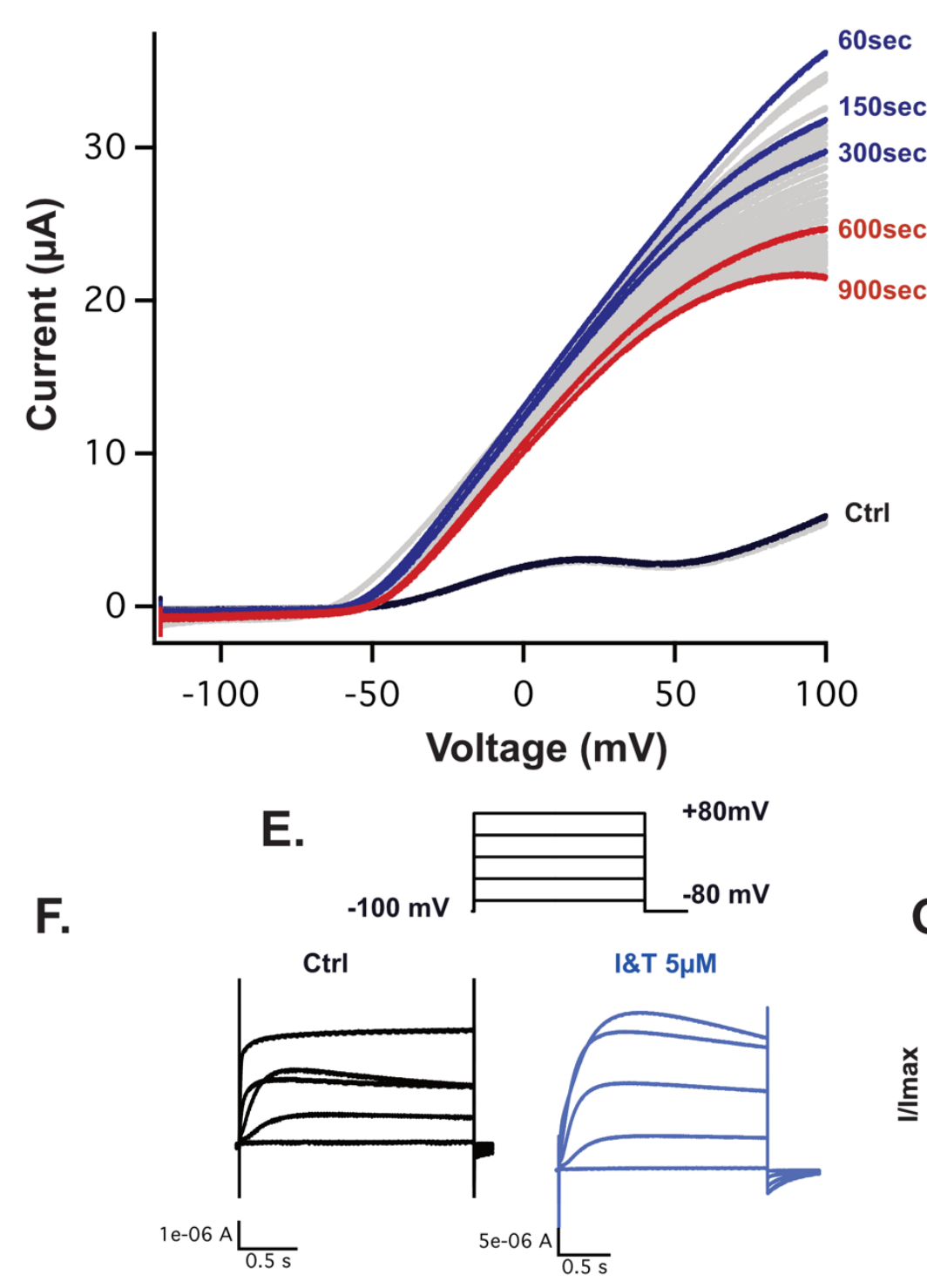

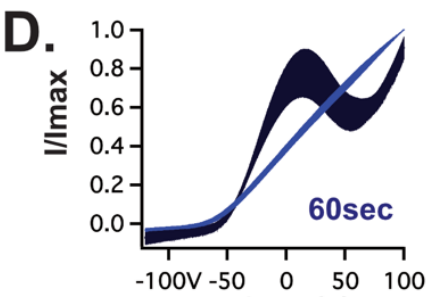

Voltage (mV)

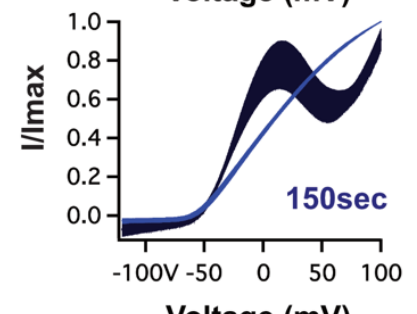

Voltage $(\mathrm{mV})$

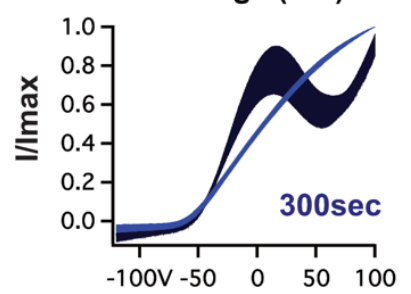

Voltage $(\mathrm{mV})$

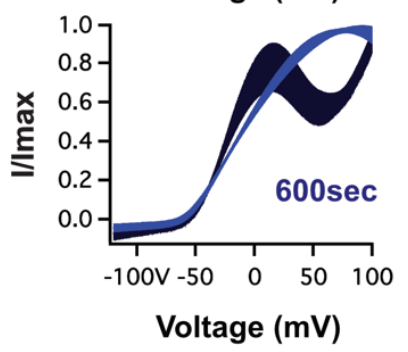

G.

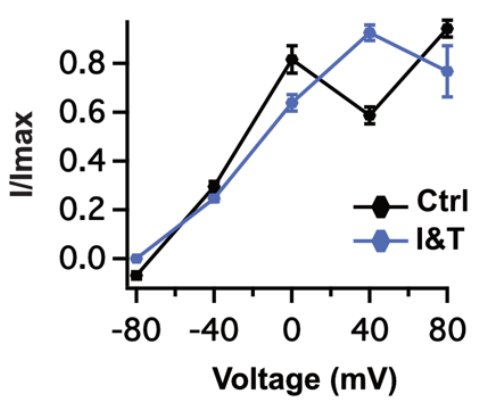

Figure 31. $\mathrm{Ca}^{2+-}$ Calmodulin potentiates E600R current converting the biphasic IV to a linear one. A) Ramp protocol (5s) repeatedly applied with a 30s interval. No leak subtraction B.) Representative current response to the ramp protocol in A. $5 \mu \mathrm{M}$ ionomycin and thapsigargin (I\&T) were applied. Blue traces are within 300s while red traces exceed 300s. Normalized current vs. voltage of the ramp (IV) ( $n=5 ; \pm$ SEM) for C) Ctrl current (dark blue) D) Time points (60, 150 300,600 s) (lighter blue) E) Voltage steps applied after $200 \mathrm{~s}$ of applying $5 \mu \mathrm{M}$ I\&T; 2 s pulses from +80 to -80 (decreasing order) with $40 \mathrm{mV}$ step. F) Current response G) Normalized end pulse current vs. voltage (IV) $(\mathrm{N}=10 ; \pm \mathrm{SEM})$ 


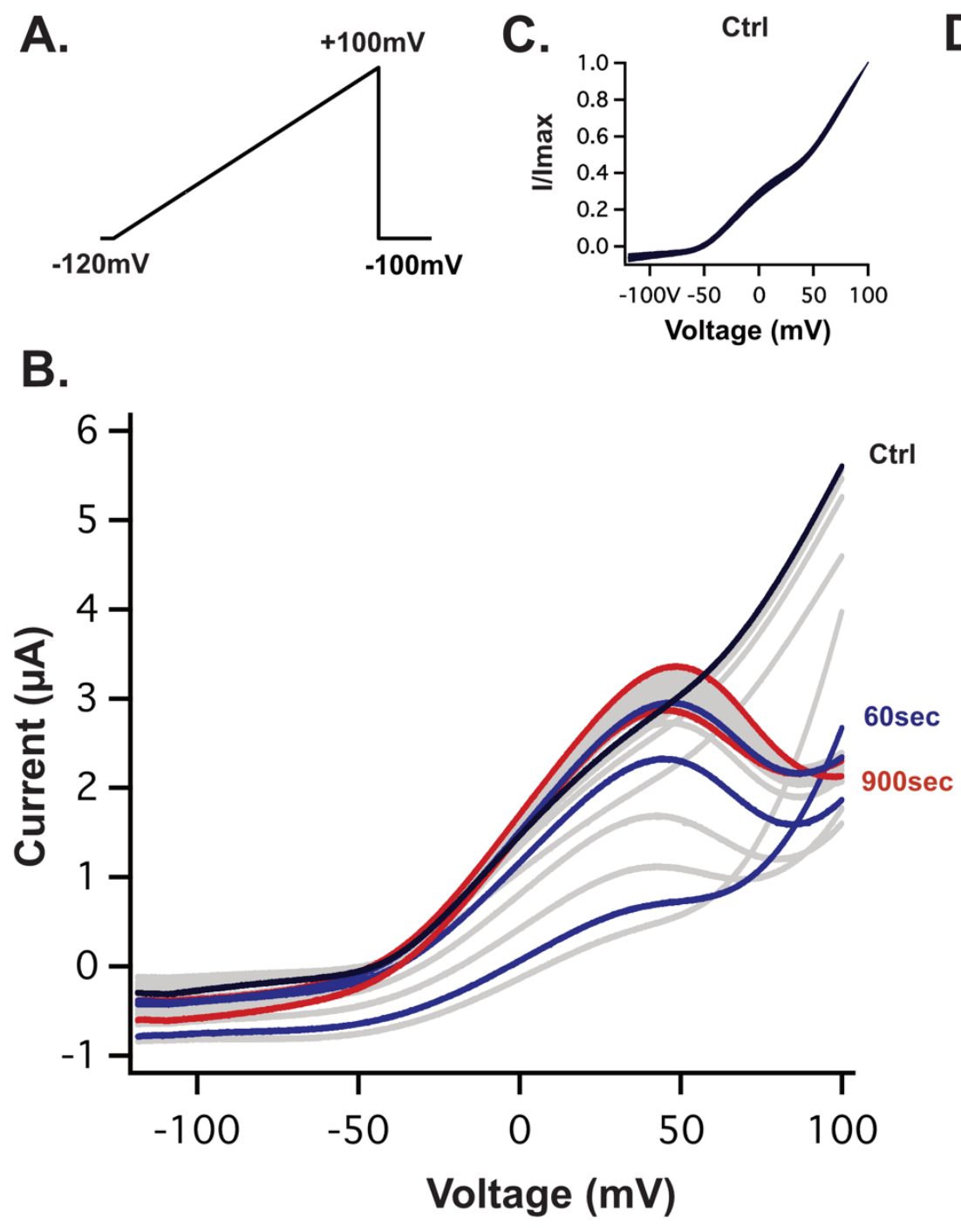

D.
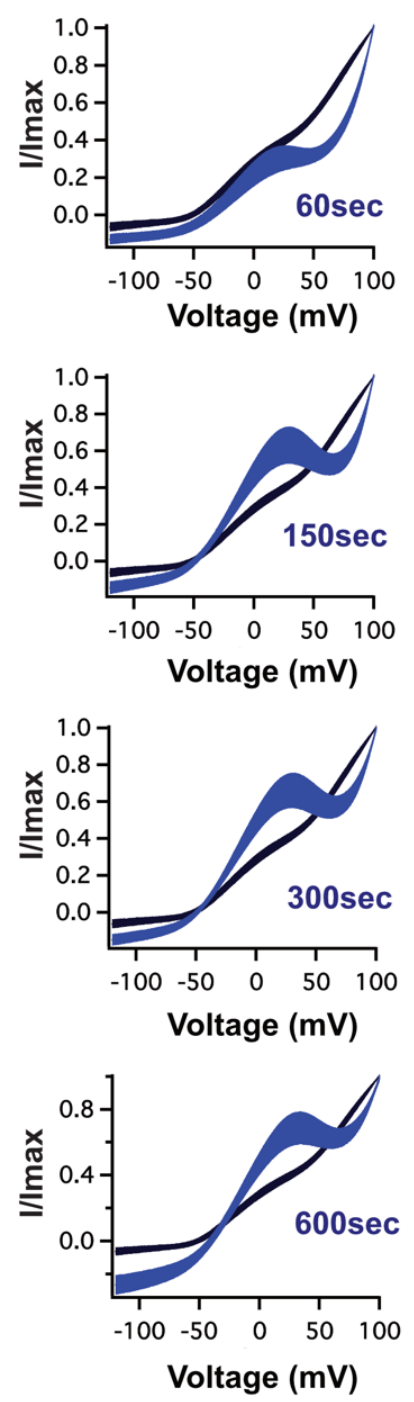

Figure 32. $\mathrm{Ca}^{2+}$-Calmodulin initially inhibits $\Delta 2-10$ then potentiates the first phase of the biphasic IV. A) Ramp protocol(5s) repeatedly applied with a $30 \mathrm{~s}$ interval. No leak subtraction B.) Representative current response to the ramp protocol in A. $5 \mu \mathrm{M}$ ionomycin and thapsigargin (I\&T) were applied. Blue traces are within $300 \mathrm{~s}$ while red traces exceed 300s. Normalized current vs. voltage of the ramp (IV) (n=6; \pm SEM) for C) Ctrl current (dark blue) D) Time points (60, 150 300,600 s) (lighter blue). 


\subsubsection{The first phase of the biphasic IV is lost when the Calmodulin- binding site BD-C2 is disrupted}

Intracellular concentration of $\mathrm{Ca}^{2+}$ ions in the oocytes is around $100 \mathrm{nM}$ (Cork et al., 1987). A slight increase of $\mathrm{Ca}^{2+}$ levels suffice to inhibit $\mathrm{K}_{\mathrm{v}} 10.1$; IC $\mathrm{I}_{50}$ for $\mathrm{Ca}^{2+}{ }^{2+}$ Calmodulin inhibition is $100 \mathrm{nM}$ (Schönherr et al., 2000). Calmodulin may stabilize $\mathrm{O}_{1}$ at basal $\mathrm{Ca}^{2+}$ level. To test this possibility, we insert mutations into the Calmodulin binding sites at both $\mathrm{N}$ - and C- terminus (BD-N and BD-C2) of $\Delta 2-10$ (Fig. 33) and examined their respective GV. Voltage steps from $-100 \mathrm{mV}$ to $+120 \mathrm{mV}$ were applied. Recordings were performed in $60 \mathrm{mM} \mathrm{K}^{+}$solution. Conductance was then calculated using end pulse current (Eq1), and the GV relationship was then fitted with Eq4 and Eq5. $\Delta 2-10^{\mathrm{F} 151 \mathrm{NL} 154 \mathrm{~N}}$, mutation at BD-N, showed slight attenuation of the biphasic GV. $\Delta 2-10^{\mathrm{F} 714 S F 7175}$, mutation at BD-C2, showed a monophasic sigmoidal response. In comparison to the WT, $\Delta 2-10^{\mathrm{F} 714 \mathrm{SF} 717 \mathrm{~S}}$ was shifted to the right. It was in the vicinity of the second component of $\Delta 2-10$ biphasic GV. We, therefore, interpret the loss of the biphasic GV as a loss of the first component. However, $\Delta 2-10^{\text {F714SF717S }}$ showed prominent outward rectification that resulted in small tail current amplitudes. This rectification could underlie the loss of the biphasic GV. We applied an IV protocol to exclude this possibility (Fig. 34). The IV was recorded in NFR solution where Veq for K+ is approximately $-100 \mathrm{mV}$ instead of $-20 \mathrm{mV}$ in $60 \mathrm{mM} \mathrm{K}^{+}$solution. The change of $V_{\text {eq }}$ guarantees outward current at potentials higher than $-100 \mathrm{mV}$. Under these conditions, the IV also showed a monophasic sigmoidal GV, suggesting that the mutant lost the first phase of the GV. 


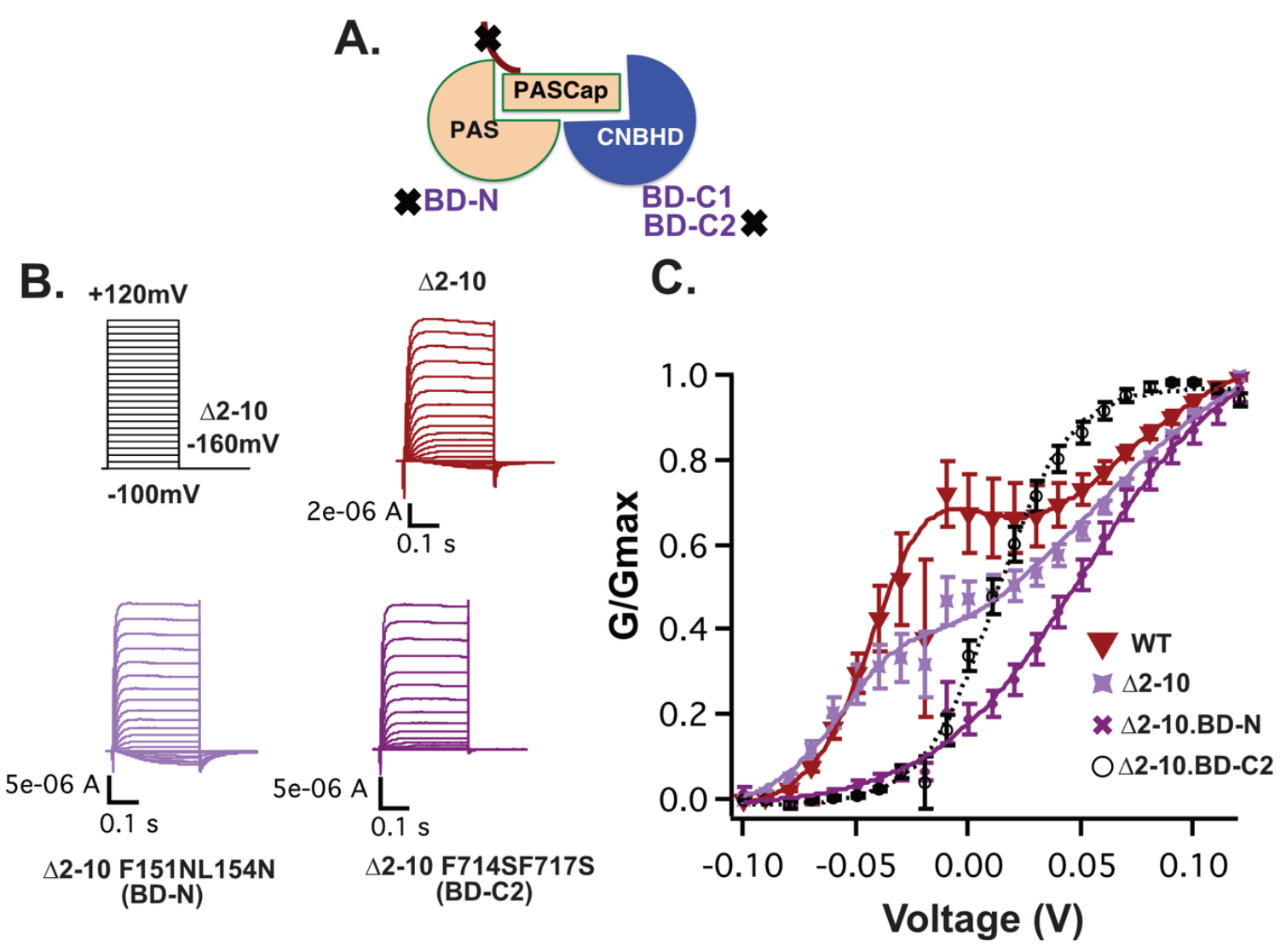

Figure 33. $\Delta 2-10$ loses the biphasic GV when BD-C2 is mutated (F714SF717S). A) Cartoon depicting mutating calmodulin binding sites (BD-N and BDC2) of $\Delta 2-10$. B) Voltage stimulus. Recording was performed in $60 \mathrm{mM} \mathrm{K}^{+}$solution. $\mathrm{P} / \mathrm{h}$ protocol was used for leak subtraction. Representative traces C) Normalized conductance vs. voltage. Conductance was calculated using end pulse current $E q$. 1. Biphasic GV curves were fitted with a double sigmoid $E q .5\left(\Delta 2-10: A_{1}=1.15, V_{1}=-0.038 \mathrm{~V}, \mathrm{~K}_{1}=0.014\right.$, $\mathrm{A}_{2}=1.09, \mathrm{Vh}_{2}=0.038 \mathrm{~V}, \mathrm{~K}_{2}=0.03, \mathrm{Vh}_{3}=0.005 \mathrm{~V}, \mathrm{~K}_{3}=0.033, \mathrm{~N}=6 ; \Delta 2-10 . \mathrm{BD}-\mathrm{N}: \mathrm{A}_{1}=1.44$, $\mathrm{Vh}_{1}=-0.038 \mathrm{~V}, \mathrm{~K}_{1}=0.014, \mathrm{~A}_{2}=1.2, \mathrm{Vh}_{2}=0.038 \mathrm{~V}, \mathrm{~K}_{2}=0.053, \mathrm{Vh}_{3}=-0.066 \mathrm{~V}, \mathrm{~K}_{3}=0.024, \mathrm{~N}=8$ ; $\Delta$ 2-10.BD-C2: $\mathrm{A}_{1}=1.1, \mathrm{Vh}_{1}=0.051 \mathrm{~V}, \mathrm{~K}_{1}=0.033, \mathrm{~N}=8$; WT: $\mathrm{A}_{1}=0.98, \mathrm{Vh}_{1}=0.012 \mathrm{~V}, \mathrm{~K}_{1}=$ $0.016 ; \mathrm{N}=11$ ) 


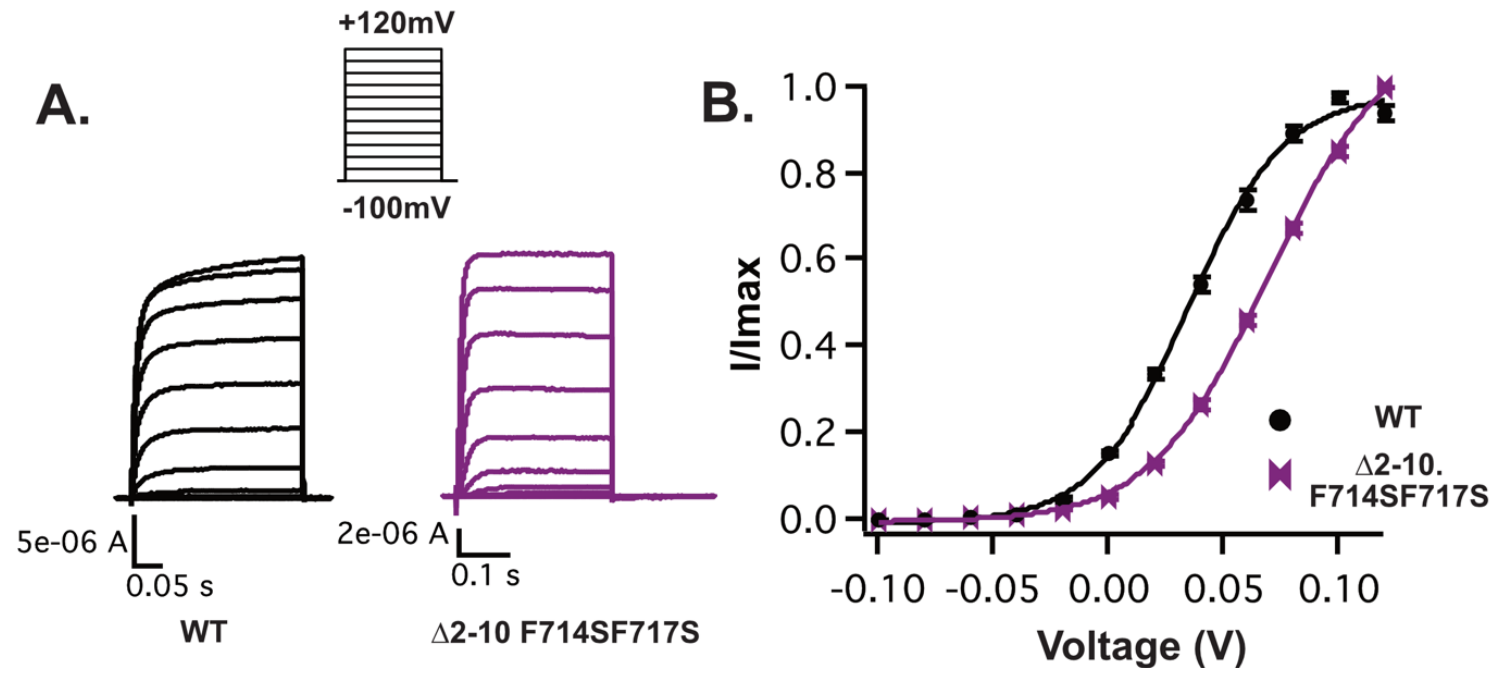

Figure 34. $\Delta 2$-10 F714SF717S monophasic IV confirms the loss of $\Delta 2-10$ biphasic GV. A) Voltage protocol applied (Upper panel). Recording performed in NFR solution. Representative traces (Lower panel). B) Normalized steady-state current vs. Voltage (IV; \pm SEM). IV fitted with a sigmoid function. WT: $A_{1}=0.99, V_{1}=0.035 \mathrm{~V}, \mathrm{~K}_{1}=0.021$, $\left.\mathrm{N}=14 ; \Delta 2-10 . \mathrm{BD}-\mathrm{N}: \mathrm{A}_{1}=1, \mathrm{Vh}_{1}=0.07 \mathrm{~V}, \mathrm{~K}_{1}=0.025\right), \mathrm{N}=6$ )

We then mutated the Calmodulin binding sites of $\triangle$ PASCap and examined its GV (Fig. 35). G was calculated using end-pulse current Eq1 except for $\Delta$ PASCap ${ }^{\text {F714SF717S, }}$ tail current was used Eq2 and Eq3. The first phase of the biphasic GV of $\triangle$ PASCap ${ }^{\mathrm{F} 151 \mathrm{NL} 154 \mathrm{~N}}$, mutation at BD-N, was amplified. $\Delta$ PASCap ${ }^{\mathrm{F} 714 S F 717 \mathrm{~S}}$, modification at BDC2, showed a monophasic sigmoidal response. In comparison to the WT, $\triangle$ PASCap ${ }^{\text {F714SF717S }} \mathrm{GV}$ was shifted to the right. It was close to the second component of $\triangle$ PASCap biphasic GV. The loss of the biphasic GV could correspond to a loss of the first component. As a control, Calmodulin binding sites of the WT were also mutated (Fig. 36). $\mathrm{WT}^{\mathrm{F} 151 \mathrm{NL} 154 \mathrm{~N}}$ showed a biphasic response, which is less prominent and not clearly visible as the $\mathrm{N}$-terminal deletions. WT ${ }^{\mathrm{F} 714 \mathrm{SF} 717 \mathrm{~S}}$ almost overlapped with WT with a slight change in slope. 


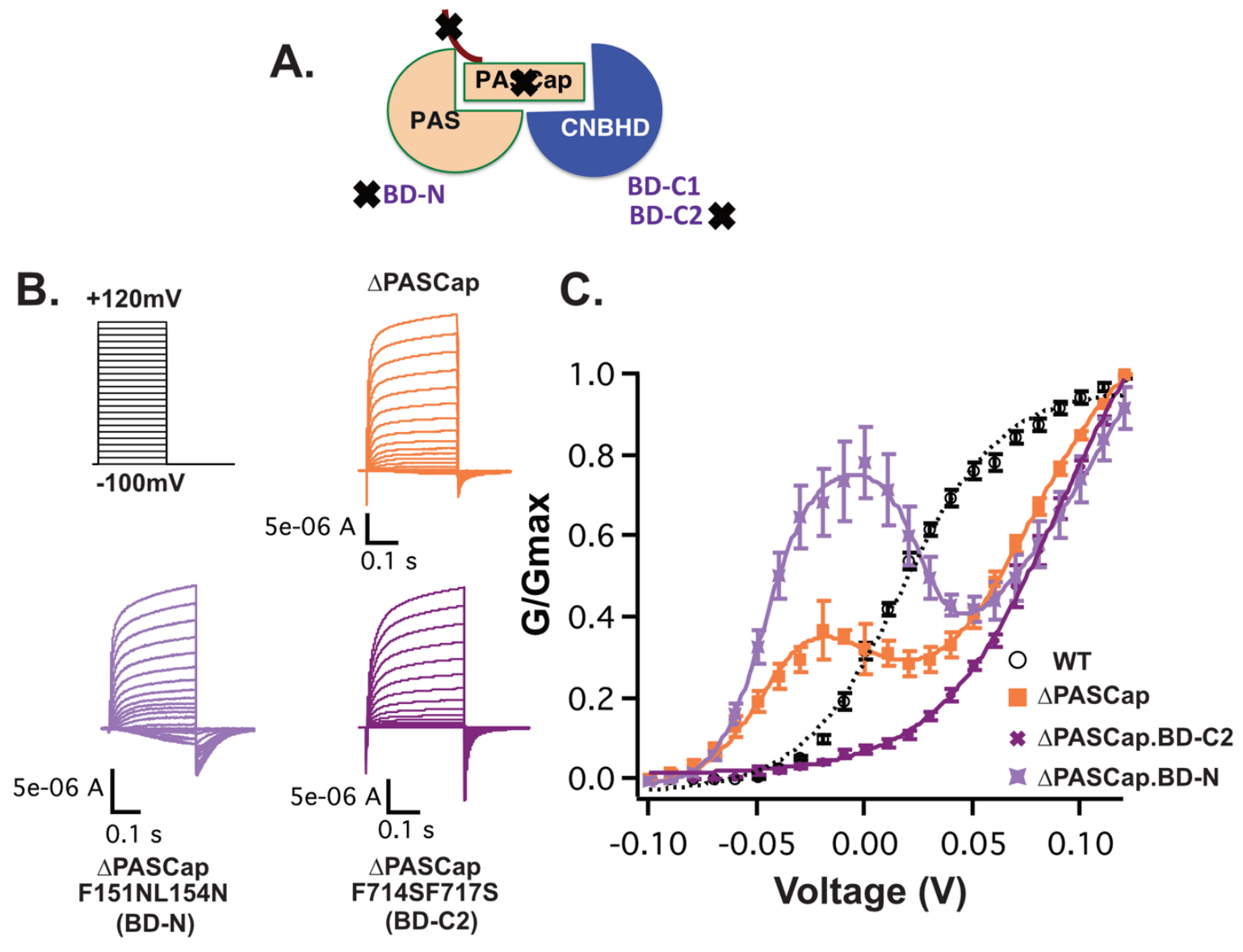

Figure 35. $\triangle$ PASCap loses the biphasic GV when BD-C2 is mutated (F714SF717S). A) Cartoon depicting mutating calmodulin binding sites (BD-N and BDC2) of $\triangle$ PASCap. B) Voltage stimulus. Recording was performed in $60 \mathrm{mM} \mathrm{K}^{+}$solution. $\mathrm{P} / \mathrm{n}$ protocol was used for leak subtraction. Representative traces C) Normalized conductance plotted $v s$. voltage (GV; \pm SEM). Conductance was calculated using end pulse current (Eq. 1) ( $\triangle$ PASCap, $\triangle$ PASCapF151NL154N) and tail current (WT, $\triangle$ PASCap F714SF717S) (Eq. 2, $E q$. 3). Biphasic GV curves were fitted with a double sigmoid. ( $\triangle$ PASCap: $A_{1}=1.15, V_{1}=$ $-0.038 \mathrm{~V}, \mathrm{~K}_{1}=0.014, \mathrm{~A}_{2}=1.17, \mathrm{Vh}_{2}=0.072 \mathrm{~V}, \mathrm{~K}_{2}=0.026, \mathrm{Vh}_{3}=-0.036 \mathrm{~V}, \mathrm{~K}_{3}=0.036, \mathrm{~N}=6$; $\triangle$ PASCap.BD-N: $\mathrm{A}_{1}=0.8, \mathrm{Vh}_{1}=-0.046 \mathrm{~V}, \mathrm{~K}_{1}=0.011, \mathrm{~A}_{2}=2.6, \mathrm{Vh}_{2}=0.16 \mathrm{~V}, \mathrm{~K}_{2}=0.06$, $\mathrm{Vh}_{3}=0.027 \mathrm{~V}, \mathrm{~K}_{3}=0.0089, \mathrm{~N}=7$; $\triangle$ PASCap.BD-C2: $\mathrm{A}_{1}=1.32, \mathrm{Vh}_{1}=0.09 \mathrm{~V}, \mathrm{~K}_{1}=0.029, \mathrm{~N}=11$; WT: $A_{1}=0.99, \mathrm{Vh}_{1}=0.017 \mathrm{~V}, \mathrm{~K}_{1}=0.023, \mathrm{~N}=9$ ) 


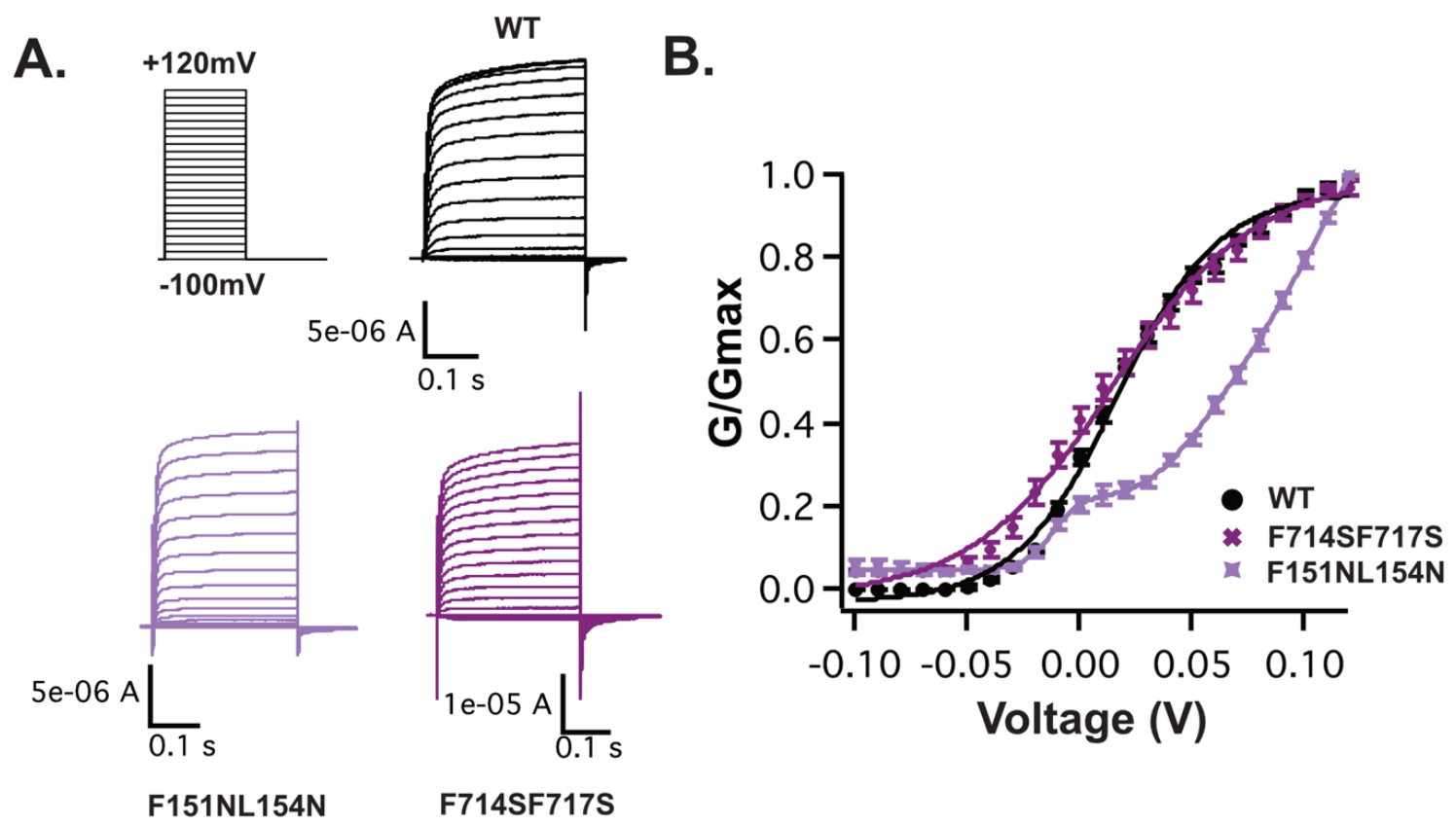

Figure 36. F714SF717S shows a similar GV to the WT. A) Voltage protocol applied. Representative current response B) Normalized conductance plotted $v s$. voltage (GV; \pm SEM). Tail fitting was used to calculate conductance (Eq. 2, Eq.)3. A sigmoid curve was used to fit GV. Biphasic GV curves were fitted with a double sigmoid. (BD-N: $A_{1}=0.19$, $\mathrm{Vh}_{1}=-0.012 \mathrm{~V}, \mathrm{~K}_{1}=0.006, \mathrm{~A}_{2}=1.96, \mathrm{Vh}_{2}=0.12 \mathrm{~V}, \mathrm{~K}_{2}=0.047, \mathrm{Vh}_{3}=0.028 \mathrm{~V}, \mathrm{~K}_{3}=0.012, \mathrm{~N}=5$; BD-C2: $\mathrm{A}_{1}=1, \mathrm{Vh}_{1}=0.015 \mathrm{~V}, \mathrm{~K}_{1}=0.032, \mathrm{~N}=8 \mathrm{WT}: \mathrm{A}_{1}=0.99, \mathrm{Vh}_{1}=0.017 \mathrm{~V}, \mathrm{~K}_{1}=0.023, \mathrm{~N}=9$ )

In addition to the GV, we checked how the mutations influenced the effect of hyperpolarizing pre-pulses on the $\triangle$ PASCap current. As shown above, $-160 \mathrm{mV}$ potentiates the current at $+40 \mathrm{mV}$ (see chapter3.3.1). Long hyperpolarizing pre-pulses (5s) were applied to a test pulse of +40 , and the current at the end of the pulse was normalized; the current corresponding to the $-20 \mathrm{mV}$ pre-pulse was used for normalizations. A remnant of the potentiation observed at $+40 \mathrm{mV}$ in response to $160 \mathrm{mV}$ pre-pulse could be detected ( $\left.\triangle \mathrm{PASCap}=3.5 \mathrm{x}, \Delta \mathrm{PASCap}^{\mathrm{F} 151 \mathrm{NL} 154 \mathrm{~N}}=1.2 \mathrm{x}\right)($ Fig. 37). $\triangle$ PASCap ${ }^{\mathrm{F} 714 S F 717 \mathrm{~S}}$ did not show current potentiation in response to hyperpolarized pre-pulses; a pre-pulse of $-160 \mathrm{mV}$ failed to potentiate the current response at moderate potential $(+40 \mathrm{mV})$. 
In summary, mutation at BD-C2 has a more significant impact on stabilizing $\mathrm{O}_{1}$ in the $\mathrm{N}$-terminal deletions $(\Delta 2-10, \Delta$ PASCap). We conclude that Calmodulin could be bound to BD-C2 at basal $\mathrm{Ca}^{2+}$ level and stabilize $\mathrm{O}_{1}$.

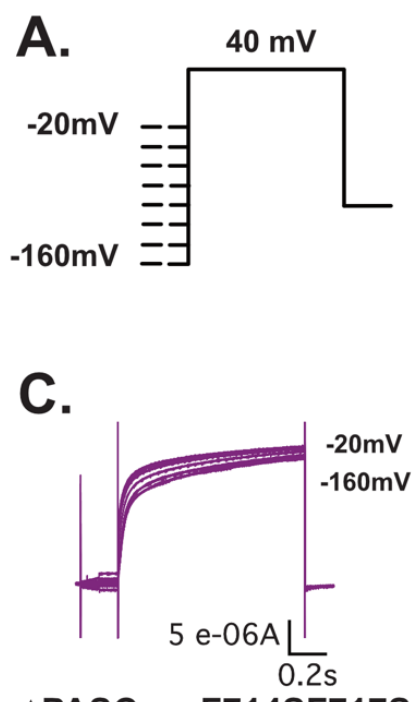

$\triangle$ PASCap. F714SF717S (BD-C2)
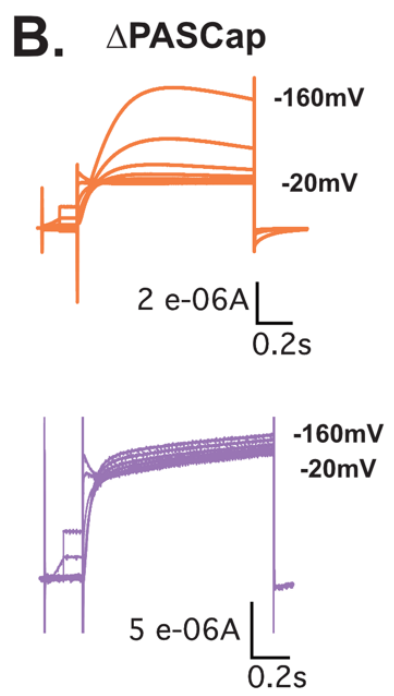

$\triangle$ PASCap. F151NL154N

(BD-N)

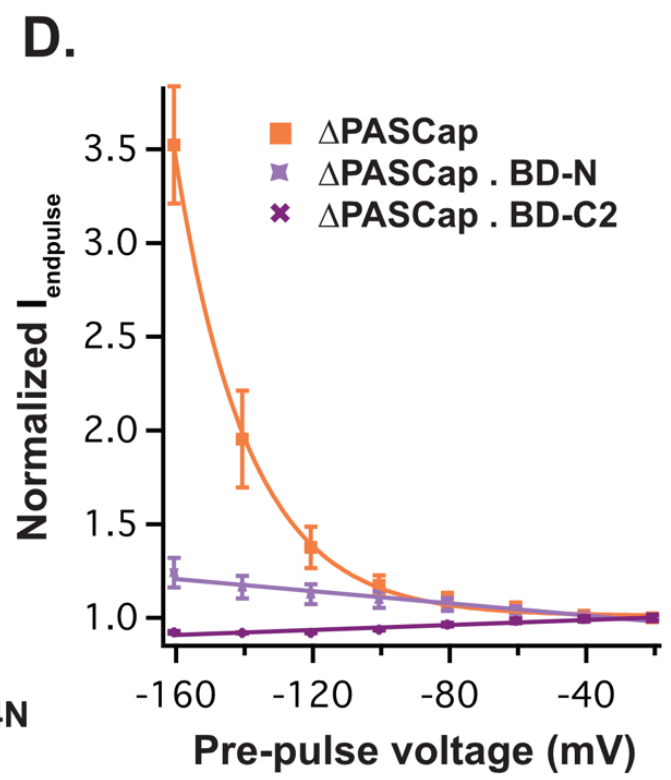

Figure 37. Hyperpolarized potentials fail to potentiate $\triangle$ PASCap current when BDC2 is mutated. A) Voltage protocol B) and C) Representative traces D) Normalized end pulse current vs. pre-pulse potential. Normalized to the response to the test pulse with $-20 \mathrm{mV}$ pre-pulse. ( $\triangle$ PASCap $\mathrm{N}=8, \Delta$ PASCap F151NL154N $\mathrm{N}=5, \Delta$ PASCap F714SF717S N=4)

\subsection{4 $\mathrm{Ca}^{2+}$ Calmodulin binding to $\mathrm{K}_{\mathrm{v}} 10.1$ at basal $\mathrm{Ca}^{2+}$ level}

To test if we indeed disrupted Calmodulin binding at basal $\mathrm{Ca}^{2+}$ level, we examined Calmodulin binding to $\mathrm{K}_{\mathrm{v}} 10.1$ at different $\mathrm{Ca}^{2+}$ concentrations. The binding of Calmodulin has been reported to be $\mathrm{Ca}^{2+}$ dependent (Schönherr et al., 2000; Ziechner et al., 2006). The binding of Calmodulin to a C-terminal fragment has been tested under different $\mathrm{Ca}^{2+}$ concentrations, and the results reported already suggested that Calmodulin could be bound at $100 \mathrm{nM} \mathrm{Ca}^{2+}$ (Schönherr et al., 2000), close to the intracellular $\mathrm{Ca}^{2+}$ concentration in Xenopus laevis oocyte, $(92.6 \pm 30 \mathrm{nM})$ (Cork et al., 1987). To test the binding of Calmodulin to $K_{v} 10.1$ full-length channel, we generated a tagged Calmodulin (5xMyc-Calmodulin). cRNA of the tagged Calmodulin was co- 
injected with $\mathrm{K}_{\mathrm{v}} 10.1$. Oocytes were lysed, and a Myc antibody was used to immunoprecipitate myc-Calmodulin. $\mathrm{K}_{\mathrm{v}} 10.1$ would be pulled down if bound to Calmodulin. The lysis and the pull-down were performed under different $\mathrm{Ca}^{2+}$ concentrations $(0,100 \mathrm{nM}, 1 \mu \mathrm{M})$. As a control, the pull-down experiment was performed on extracts from oocytes injected with Myc-Calmodulin or $\mathrm{K}_{\mathrm{v}} 10.1$ alone and from non-injected oocytes. The control groups were lysed, and the pull-down was conducted in the absence of $\mathrm{Ca}^{2+}$. Proteins from the pull-down were loaded on a gel, separated by electrophoresis and transferred to a nitrocellulose membrane. The membrane was incubated with an antibody against $\mathrm{K}_{\mathrm{v}} 10.1$ to detect the channel protein in the different groups (Fig. 38). $\mathrm{K}_{\mathrm{v}} 10.1$ band appeared at $100 \mathrm{nM}$ and $1 \mu \mathrm{M}$. The band was around $110 \mathrm{Kd}$ as previously reported (Napp et al., 2005). This result supports the possibility of Calmodulin binding to $\mathrm{K}_{\mathrm{v}} 10.1$ at basal $\mathrm{Ca}^{2+}$.
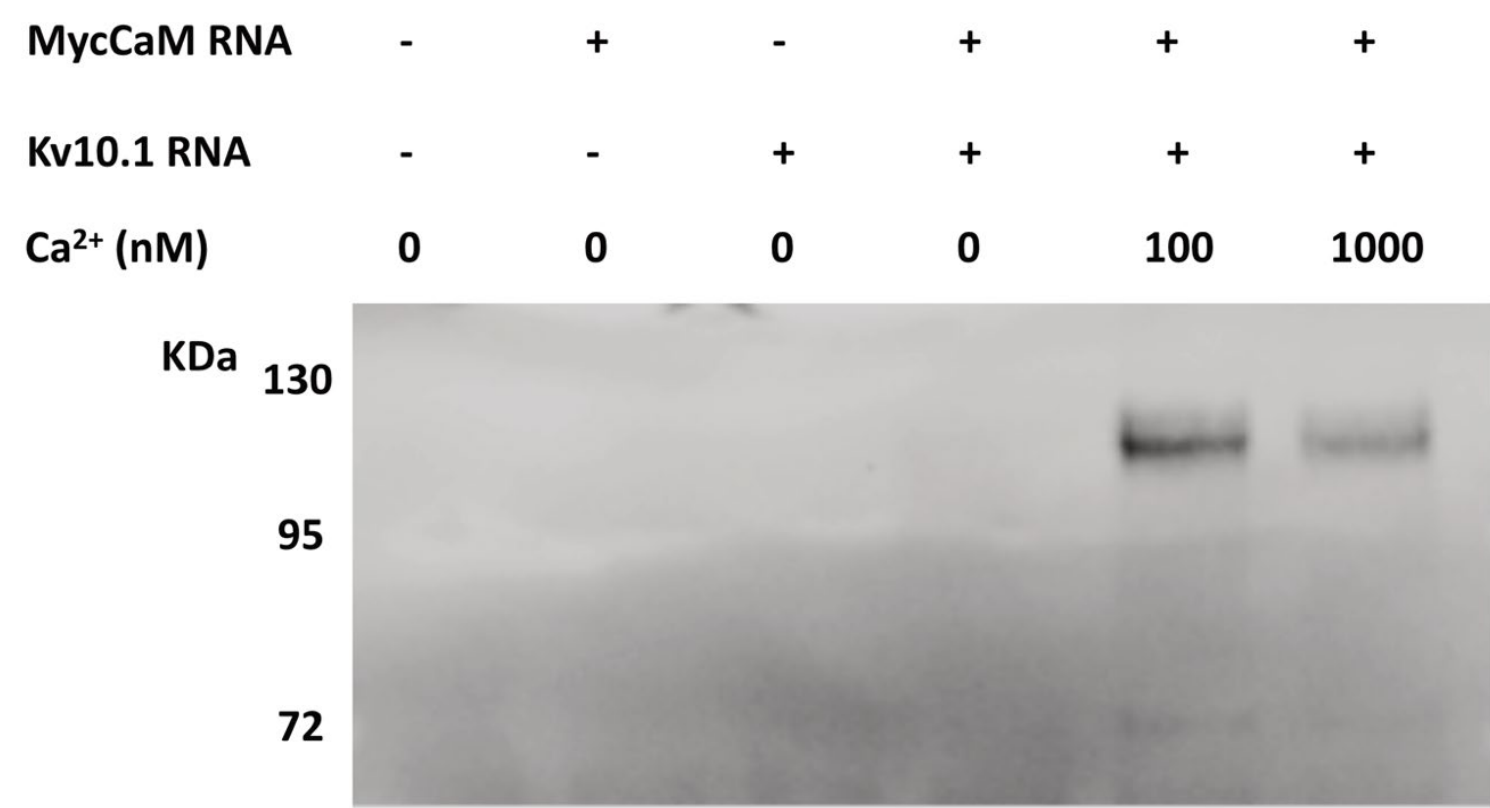

Figure 38.Pull-down of Kv10.1 with 5xmyc-Calmodulin at 100nM Ca2+. Xenopus laevis oocytes were co-injected with $5 x$ myc-Calmodulin and $\mathrm{K}_{\mathrm{v}} 10.1$. 5xmyc-calmodulin was immunoprecipitated with anti-myc antibody. The immunoblot was probed with anti-Kv10.1. Control groups (3 lanes on the left) in order: non-injected oocytes, oocytes injected with $5 x$ myc-calmodulin, oocytes injected with $\mathrm{Kv} 10.1 . \mathrm{Ca}^{2+}$ was absent in the control groups. Oocytes injected with 5xmyc-Calmodulin and $\mathrm{K}_{\mathrm{v}} 10.1$ (3 lanes on the right). Three $\mathrm{Ca}^{2+}$ concentrations $(0,100 \mathrm{nM}, 1 \mu \mathrm{M}) . \mathrm{K}_{\mathrm{v}} 10.1$. band was observed at the expected size with $100 \mathrm{nM}$ and $1 \mu \mathrm{M} \mathrm{Ca}^{2+}$. 


\subsection{Second gating step: A mutation at the end of S4 (D342C) reproduces the biphasic behavior (shifts $\mathrm{O}_{2}$ to the right)}

The D342C mutant also showed a biphasic GV, although not as dramatic as that observed with the N-terminus deletions. The first component overlayed with WT, while the second component was shifted to the right. Fitting the normalized GV with Eq5 has yielded two components and a transition component, as shown in Fig. 39. We named the component with the open state we suggest it represents (first component; $\mathrm{O}_{1}$, second component; $\mathrm{O}_{2}$ ). The fitting gives $\mathrm{O}_{1}$ higher conductance than $\mathrm{O}_{2}$. The transition started at potentials earlier than $\mathrm{O}_{1}$ and $\mathrm{O}_{2}$ and saturated at $0 \mathrm{mV}$.

A sigmoidal activation in response to $-160 \mathrm{mV}$ pre-pulse and external $\mathrm{Mg}^{2+}$ characterizes $\mathrm{K}_{\mathrm{v}}$ 10.1. This sigmoidal activation is compromised by the D342C mutation; the activation was slowed down in response to $-160 \mathrm{mV}$ pre-pulse without a detectable sigmoidal phase (Fig. 40). Rise time ( $\mathrm{t}=80 \%)$, time to reach $80 \%$ of maximum current, was calculated at negative potential to estimate activation kinetics. At $-160 \mathrm{mV}$ pre-pulse WT has a larger risetime in comparison to D342C (risetime $80 \%$ : $\mathrm{WT}=0.25 \pm 0.011, \mathrm{D} 342 \mathrm{C}=0.12 \pm 0.012)$. Risetime $(\mathrm{t}=80 \%)$, is plotted against prepulse potential as an estimate of the change in kinetics. D342C showed a similar acceleration to the WT, with a slight shift to the left $(\mathrm{WT} V h=-0.09, \mathrm{D} 342 \mathrm{C}$ Vh $=-0.07$; SEM). 


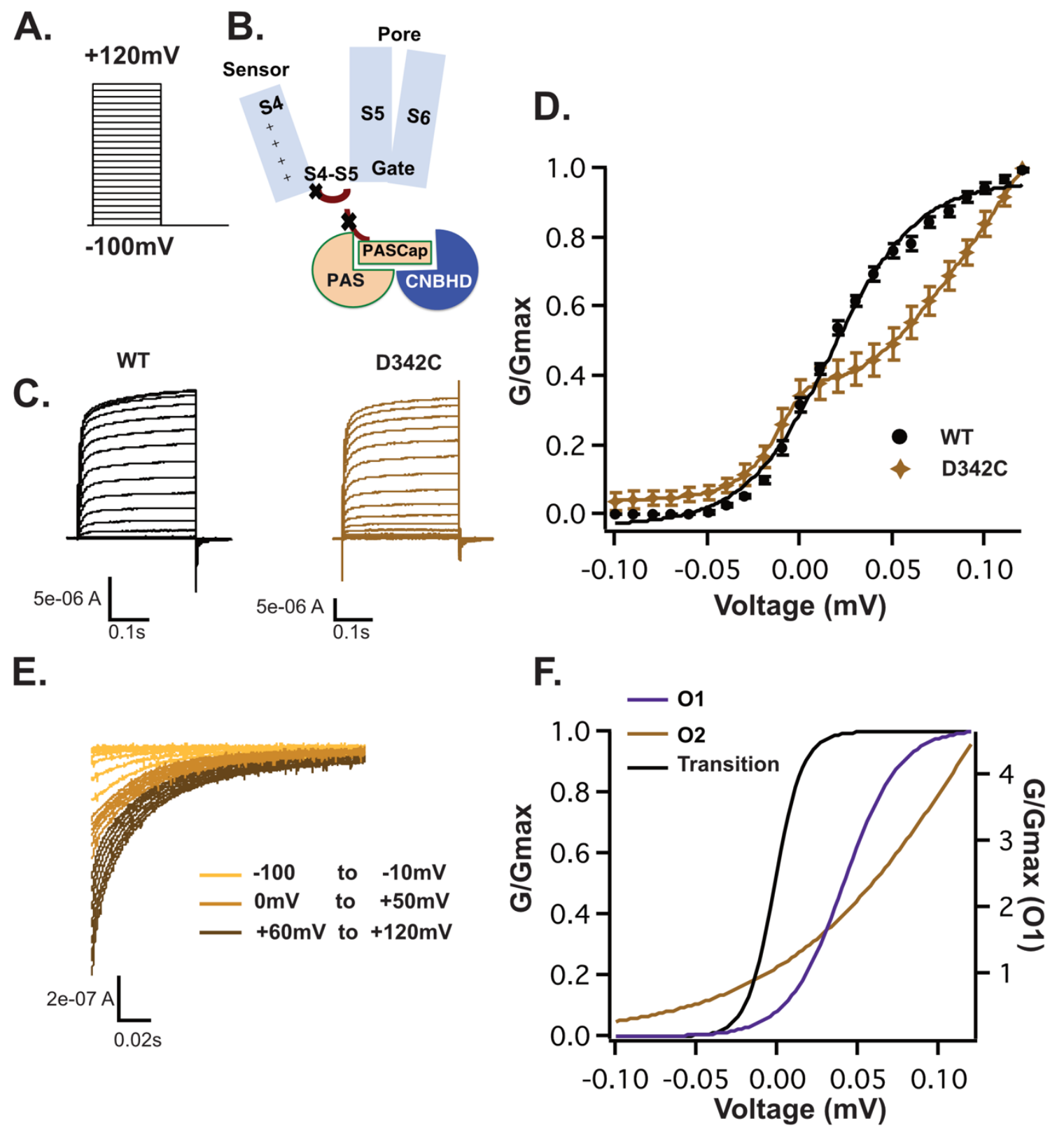

Figure 39. D342C induces biphasic behavior in Kv10.1 A) Voltage stimulus applied; $\Delta 10 \mathrm{mV}$ steps B) Cartoon of mutant C) Representative traces. D) Normalized conductance vs. voltage. (Tail analysis Eq. 2 and Eq. 3) (WT N=9, D342C N=6; \pm SEM). WT is fitted with one sigmoid $(\mathrm{A} 0=-0.0311681, \mathrm{~A} 1=0.993368, \mathrm{Vh} 1=0.0167173, \mathrm{~K} 1=$ 0.0230901). E) Tail kinetics in C.) F) Individual GV components using Eq. 3. $1^{\text {st }}$ component (A1) represents $0_{1}$, shown in purple. $2^{\text {nd }}$ component represents (A2) $\mathrm{O}_{2}$, shown in gold. Transition between the two components (weight) is in black. ( $\mathrm{A}_{0}=0.04$, $\mathrm{A}_{1}=4.66, \mathrm{Vh}_{1}=0.041 \mathrm{~K}_{1}=0.017 ; \mathrm{A}_{2}=2.04, \mathrm{Vh}_{2}=0.127, \mathrm{~K} 2=0.061 ; \mathrm{Vh}_{3}=-0.0016, \mathrm{~K}_{3}=$ $0.0086)$ 

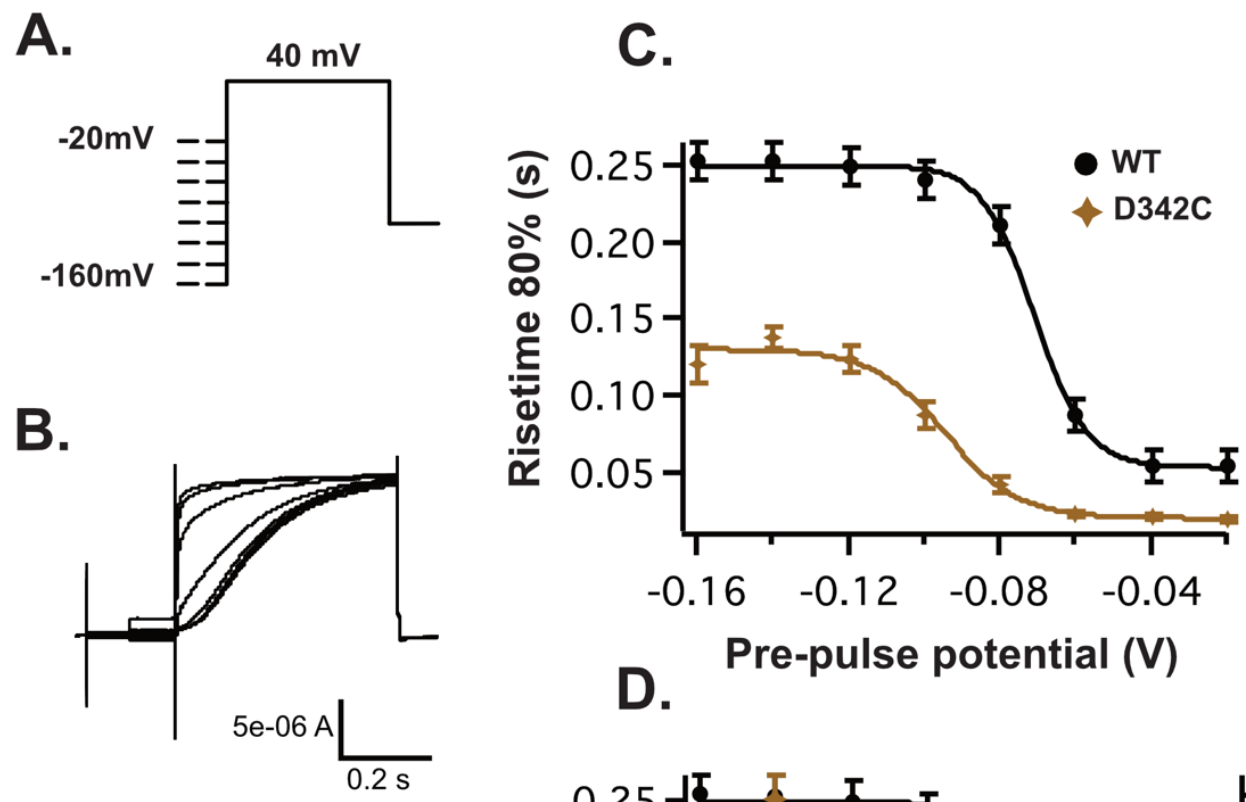

\section{D.}

Pre-pulse potential (V)

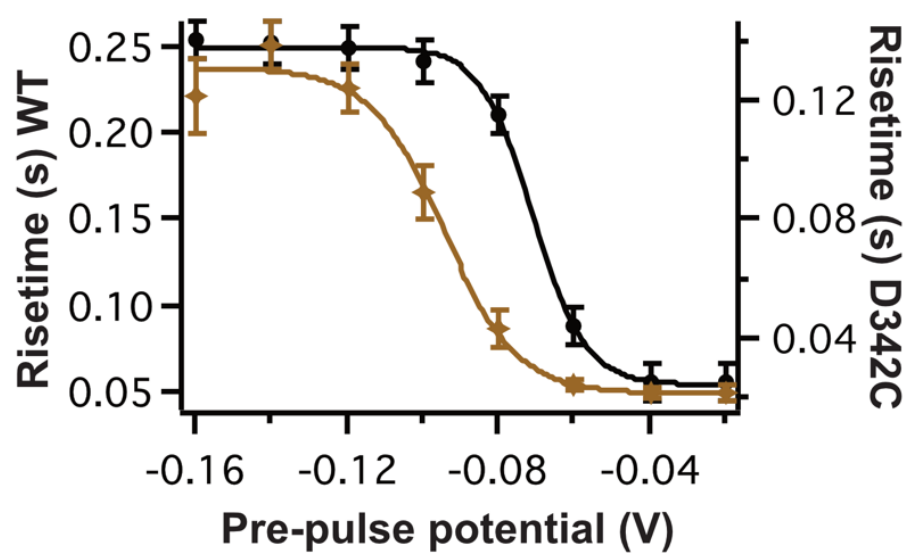

Figure 40. D342C Cole-Moore is shifted to the left. A) Cole-Moore protocol. $5 \mathrm{mM}$ $\mathrm{Mg}^{2+}$ NFR solution was perfused. B) Representative traces C) Risetime ( $\mathrm{t}=80 \%$ ) vs. prepulse potential (WT N=6; D342C N=5) D) Sigmoidal Fit (WT Vh= -0.09, D342C Vh= $0.07 ; \pm$ SEM)

\section{5 $\triangle \mathrm{CNBHD}$ requires stronger depolarizations to open and loses Cole-Moore}

In contrast to the $\mathrm{N}$-terminal deletions, the C-terminal deletion (525-697) retained a monophasic response. However, the GV was shifted to the right, and the sigmoidal saturation could not be observed within the tested voltage range (Fig. 41C). We examined the Cole-Moore phenomenon for the C-terminal deletion. Long hyperpolarizing pre-pulses were used. $5 \mathrm{mM} \mathrm{Mg}^{2+}$ was used to potentiate the effect of hyperpolarizing potentials. The rise time $(\mathrm{t}=805)$ remained almost constant in 
response to the different pre-pulses. In contrast, the WT showed a decrease in rise time at $-80 \mathrm{mV}$ and $-60 \mathrm{mV}$ (Cole-Moore). $\triangle \mathrm{CNBHD}$ did not show Cole-Moore effect; depolarized pre-pulses failed to accelerate activation (Fig. 41D and E).

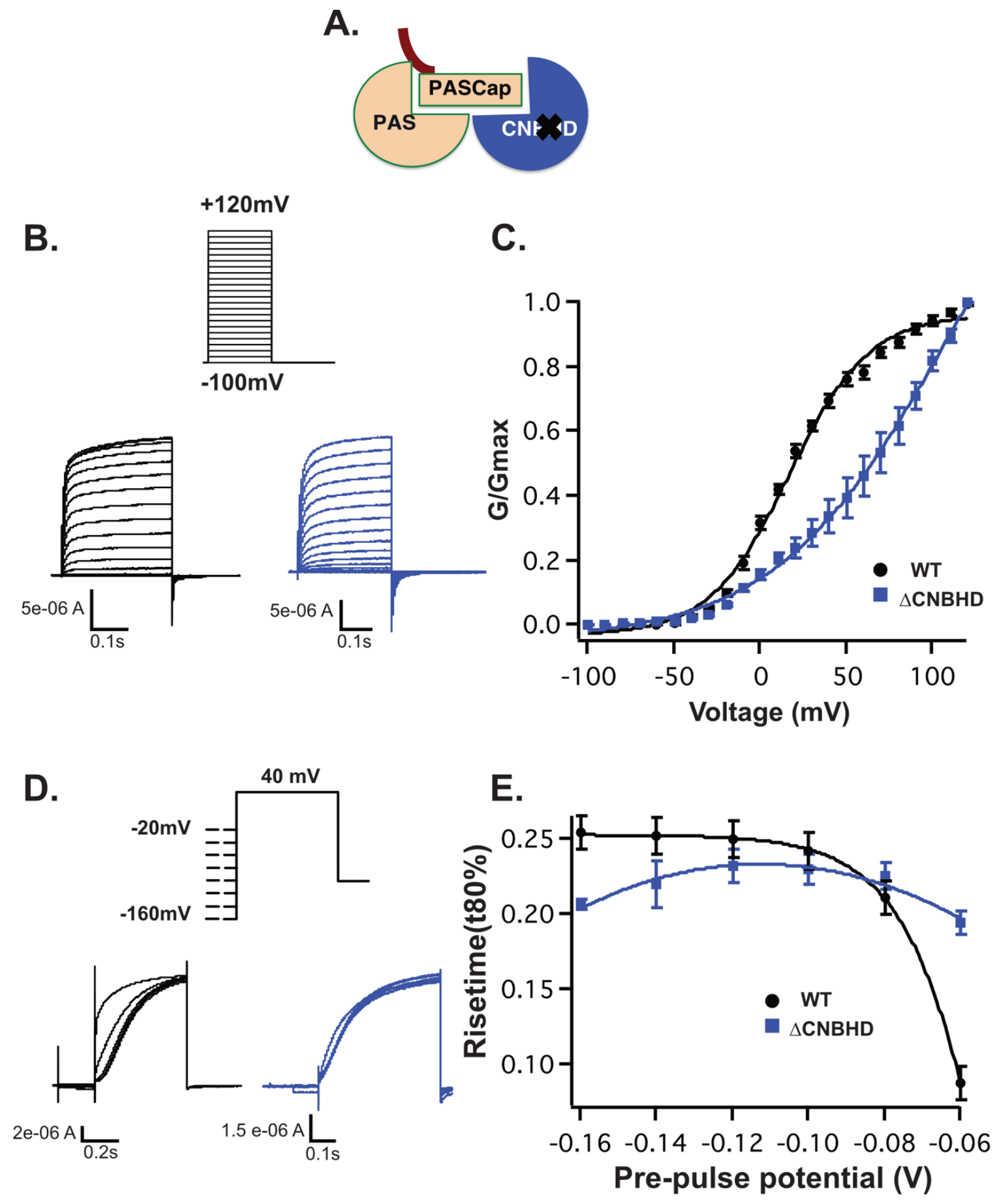

Figure 41. $\triangle$ CNBHD shifts GV to the right and loses Cole-Moore. A) $\triangle \mathrm{CNBHD}$ cartoon B) Voltage stimulus applied. Representative current traces: WT, $\triangle$ CNBHD. $60 \mathrm{mM} \mathrm{K}^{+}$solution was perfused. C) Normalized conductance $v s$. voltage. Conductance calculated using tail fitting Eq. 2 and Eq. 3. GV fitted with sigmoid Eq. 4 WT Vh= 16.7mV, $\mathrm{K}_{1}=23 \mathrm{~N}=9$; $\Delta \mathrm{CNBHD} \mathrm{Vh}_{1}=108 \mathrm{mV}, \mathrm{K}_{1}=50, \mathrm{~N}=5$; $\pm \mathrm{SEM}$ ). D) Cole-Moore protocol. $5 \mathrm{mM} \mathrm{Mg}^{2+}$ NFR solution was perfused. Representative traces: WT, $\triangle$ CNBHD. 
E) Time to reach $80 \%$ of maximum current (risetime80\%) vs. pre-pulse potential (WT $\mathrm{N}=6 ; \triangle \mathrm{CNBHD} \mathrm{N}=4 ; \pm \mathrm{SEM}$ ).

\subsection{Appendix}

\subsubsection{WT tail current kinetics is slower with a weak depolarizing pre- pulse $(-20 \mathrm{mV})$}

We examined the change in tail current kinetics in response to a range of prepulses (from $-160,-80 \mathrm{mV}$ and $-20 \mathrm{mV}$ ). We also varied the duration of the test pulse to monitor any change in kinetics with time. A test pulse of $+40 \mathrm{mV}$ was used and oocytes were perfused with $60 \mathrm{mM} \mathrm{K}^{+}$solution. Tails were fitted with double exponential Eq2, and two time-constants (fast and slow) were plotted against the duration of the test pulse. The fast time constant $\left(\tau_{1}\right)$ was the largest in response to $-20 \mathrm{mV}$. A pre-pulse of $-80 \mathrm{mV}$ had a larger tau1 than $-160 \mathrm{mV}$. The slow time constant $\left(\tau_{2}\right)$ was largest in response to $-20 \mathrm{mV}$, while it was almost unchanged between $-160 \mathrm{mV}$ and $-80 \mathrm{mV}$. In summary, a pre-pulse of $-20 \mathrm{mV}$ showed slower tail current kinetics than the pre-pulse of $-160 \mathrm{mV}$. The change in tail current kinetics could indicate that the path of deactivation is different. The WT might have access to more than one open state (Fig. $42)$. 


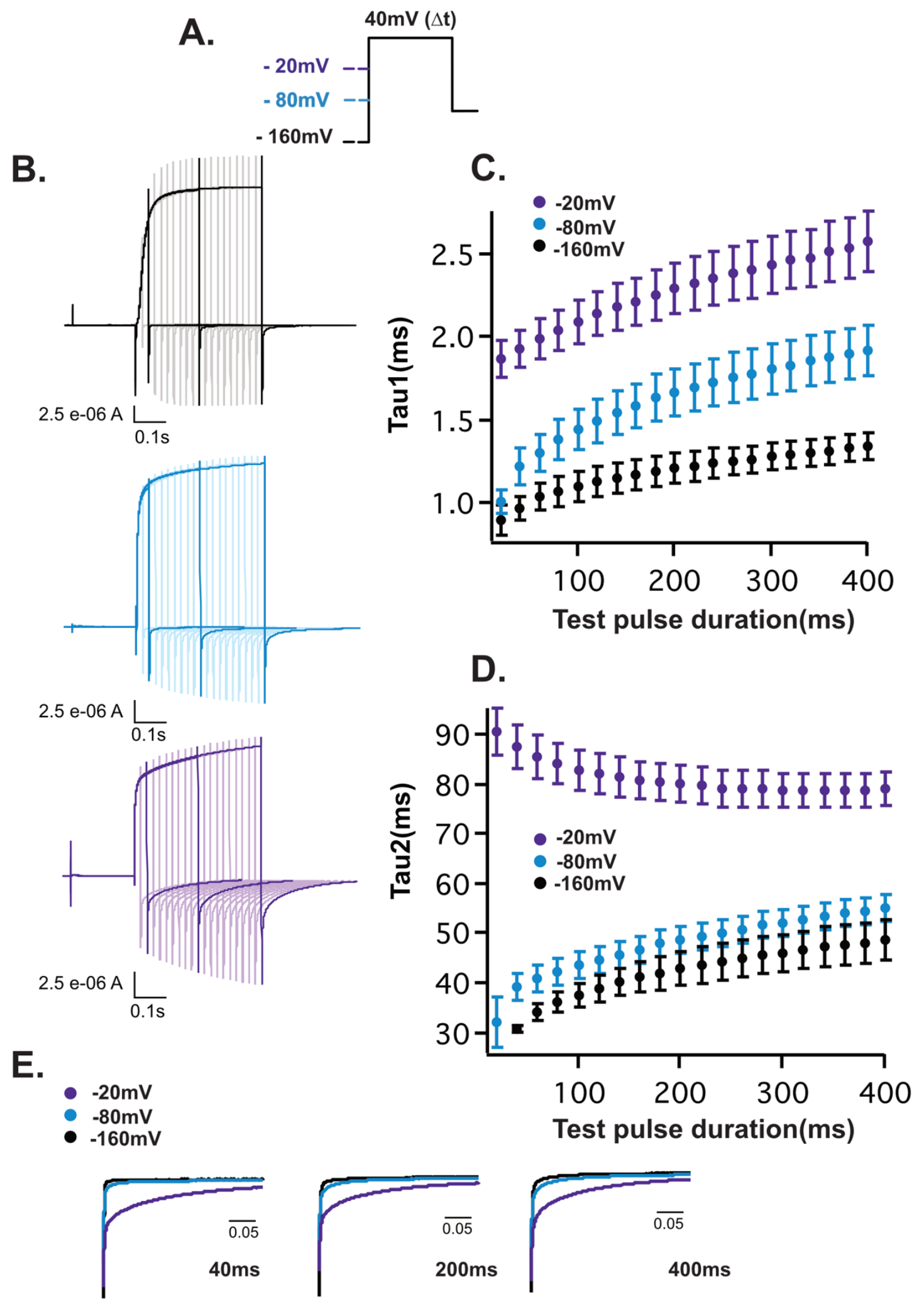

Figure 42. Depolarized pre-pulse slows down the deactivation of WT. A) Voltage protocol applied. Pre-pulse for $5 \mathrm{~s}, \Delta \mathrm{t}=20 \mathrm{~s}$ sec.B) Representative traces C) Time constants (Tau1, Tau2) vs. duration of test potential $(-20 \mathrm{mV} \mathrm{N}=11 ;-80 \mathrm{mV} \mathrm{N}=8$; $160 \mathrm{mV} \mathrm{N}=5 ; \pm$ SEM) E) Representative tail kinetics. 


\section{Discussion}

In this study, we investigate the electromechanical coupling of $\mathrm{K}_{\mathrm{v}} 10.1$. We explore an alternative gating mechanism to the mechanical lever model, which has failed to explain the gating events of the $K C N H$ family (de la Peña et al., 2018; Lorinczi et al., 2015; Tomczak et al., 2017; Wang and MacKinnon, 2017; Whicher and MacKinnon, 2016). We have focused on the N-terminus, which lies in a cavity lined by important molecular players and could play a role in orchestrating the gating events (Whicher and MacKinnon, 2016). We provide evidence for the importance of the flexibility of the N-terminus in stabilizing an open state. Then, we identify an interaction surface between the PASCap and CNBHD, which could stabilize a closed state. The disruption of this interface (N-terminal deletions, E600R) has revealed an open state, which we termed $\mathrm{O}_{1}$. The mutants, therefore, showed a biphasic behavior that corresponds to two open states $\left(\mathrm{O}_{1}\right.$ and $\left.\mathrm{O}_{2}\right)$ with distinct voltage-dependence and kinetics. We suggest that the two states have different conductance and are probably not connected in a linear pathway. We propose a model where the movement of the voltage sensor and the opening of the intracellular gating ring grant access to $\mathrm{O}_{1}$ and $\mathrm{O}_{2}$, respectively. Finally, we suggest that Calmodulin is a part of the gating machinery of $\mathrm{K}_{\mathrm{v}}$ 10.1. It is probably bound at basal $\mathrm{Ca}^{2+}$ levels at BD-C2. The binding of Calmodulin stabilizes the access to the open state " $\mathrm{O}_{1}$ " in the mutants. We will dissect each of these findings, then review the possible role of the different domains in the intracellular gating ring.

\subsection{Mobility of the initial $\mathrm{N}$-terminus is crucial for the gating events of $K_{v} 10.1$}

We initially aimed to confirm the N-terminus' proximity to critical molecular players, as suggested by the Cryo-EM structure. Introducing a Cys residue at the initial $\mathrm{N}$-terminus (R8C) could assess its proximity to endogenous Cys residues. The oxidizing 
reagent $\mathrm{TbHO}_{2}$ was used to form disulfide bridges between $\mathrm{R} 8 \mathrm{C}$ and endogenous residues as described in (de la Peña et al., 2011; de la Peña et al., 2015), resulting in marked inhibition of the current. The published structure has captured a closed conformation of $\mathrm{K}_{\mathrm{v}} 10.1$; therefore, fixing the $\mathrm{N}$-terminus to anyone of the candidate residues would likely stabilize a closed conformation. The decline in R8C current observed when subjected to $\mathrm{TbHO}_{2}$ suggests that the S-S bridges formed stabilize a closed conformation. The effect was reverted with a reducing reagent (DTT). To identify the interaction partner of R8C, we set out to mutate endogenous Cys residues that could be close enough to position 8 . We identified the candidate residues from the cryo-EM structure. The N-terminus is close to a Cys residue at the bottom of S1 (C214) of the same subunit and two Cys residues at the adjacent subunit's CNBHD domain (C575, C640). The effect of $\mathrm{TbHO}_{2}$ was preserved in R8C.C214A and R8C.C575A.C640A. It is plausible that fixing the N-terminus to either S1 or CNBHD is sufficient to stabilize the closed conformation. Localizing the N-terminus to either position could allow residues 10-13 of the $\mathrm{N}$-terminus to interact with Tyr residues in S1 (Tyr 213) and CNBHD (Tyr639) (Fig. 43), an interaction surface recently reported to stabilize a closed conformation (Whicher and MacKinnon, 2019). Our observation underscores the significance of the N-terminal tail's flexibility since its movement seems to be required for channel opening. The $\mathrm{N}$-terminus displacement is possibly necessary to break the interaction surface between S1, CNBHD, and N-terminus and destabilize a closed state. It has been proposed that the initial N-terminus residues (R7R8) could interact with D342, moving S4, and breaking this surface of interaction (Whicher and MacKinnon, 2019). In line with our observations, a different set of interactions between the flexible $\mathrm{N}$-terminus and the gating machinery was shown to stabilize open or closed states in Kv11.1 (de la Peña et al., 2011; de la Peña et al., 2015). 

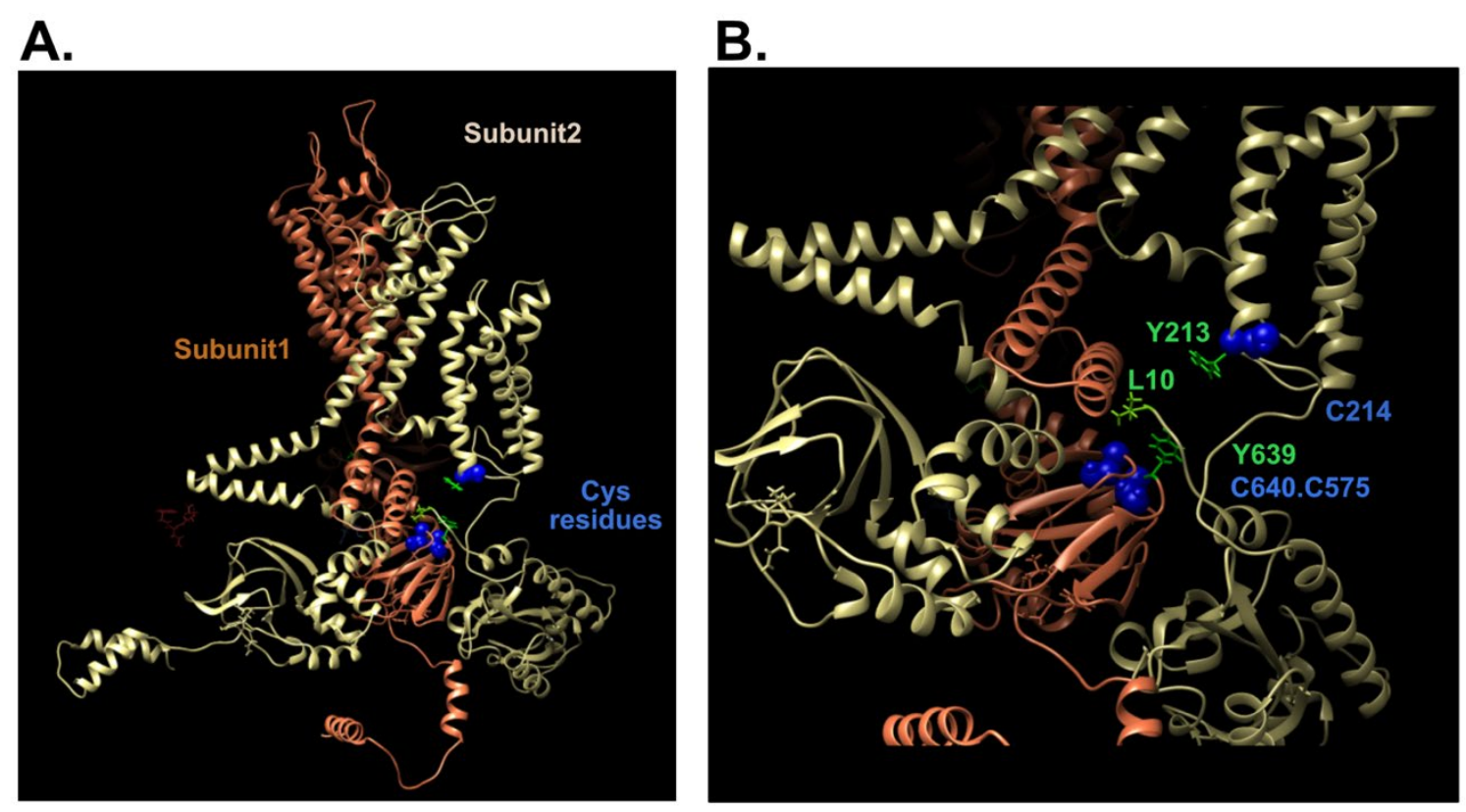

Figure 43. The N-terminus of Kv10.1 lies in the vicinity of endogenous Cys residues. Cryo-EM structure of rat $\mathrm{K}_{\mathrm{v}} 10.1$ (Whicher and MacKinnon, 2016). A) Two subunits are shown. Cys residues in blue B) Enlarged view. The three residues identified by (Whicher and MacKinnon, 2019); L10 interacts with Y213 and Y639 to stabilize a closed conformation. Cys residues that could interact with R8C: C214 (bottom of S1), C640 and C575 (CNBHD).

\subsection{Disrupting the interaction between PASCap and CNBHD reveals an open state " $0_{1}$ "}

Our interest in the $\mathrm{N}$-terminus grew, and we generated a series of $\mathrm{N}$-terminus deletions $(\Delta 2-10, \Delta \mathrm{PASCap}(2-25), \Delta \operatorname{eag}(2-135))$ and tested their conductance-voltage relationship (GV). We also tested a point mutation that disrupts the PASCap-CNBHD interaction surface (E600R) (Haitin et al., 2013; Lorinczi et al., 2016). The same residue (E627 in the mouse numbering) was first mapped to the surface of PASCap-CNBHD interaction in a crystal structure of the intracellular domain of mouse Kv10.1. E627 has been reported to influence the gating of mouse Kv10.1 (Haitin et al., 2013). E600R reverts the inhibition of $\mathrm{Kv} 10.1$ by $\mathrm{Ca}^{2+-}$ Calmodulin to potentiation (Lorinczi et al., 2016). E600I and E600L produced a similar effect, suggesting that the charge inversion between $\mathrm{E}$ and $\mathrm{R}$ is not required to disrupt the interaction surface (Lorinczi et al., 2016). 
In our hands, the N-terminal deletions and E600R showed a biphasic GV. Instead of the monophasic sigmoidal response typically observed in the WT, the mutants' GV was composed of more than one sigmoidal curve. The first sigmoidal component appeared at hyperpolarized potentials, while a second component was shifted to more depolarized potentials. The first component appeared in a similar voltage range across mutants, while the second component was variable. In between the two components, an also variable rectifying phase was observed.

We then classified the voltage ranges according to the different components of the GVs. We use the term weak depolarizing potentials when referring to the range where the first component appeared, while the strong depolarizing potentials represent a range where the second component appeared. The moderate potentials indicate the transition range. At weak depolarizations, all mutants' currents showed slow sigmoidal activation that was not observed in the WT. As the depolarizing stimulus increased, the activation kinetics was accelerated. At moderate and strong depolarization, the activation reached rates close to or identical to the WT. The behavior of deactivation kinetics was more intricate. However, a pattern common to all N-terminal deletions was that weak potentials showed slow kinetics, while strong potentials showed faster deactivation kinetics. Moderate depolarization led to a mixture of fast and slow deactivation (most conspicuously in $\triangle$ PASCap). E600R showed slow deactivation kinetics, and a peculiar rising phase was observed in the tail currents after strong potentials.

In summary, at weak potentials where the first component of the GV appeared, both activation and deactivation were slow. At strong potentials, where the second component of the GV appeared, the activation was fast and similar to WT. Deactivation kinetics was different after strong potentials. A fast component was present on all cases, but an additional component (rising phase) appeared in some cases. We do not have a definite explanation of the rising phase, which could represent the recovery of inactivation (discussed below). At moderate potentials, a transition between the two components is likely to occur. For some mutants, the kinetics appeared as a mix of fast and slow components, in the case of $\Delta$ eag for activation, for deactivation in $\triangle$ PASCap. Intriguingly a point mutation at the bottom of S4, H343R, restores slow activation and deactivation kinetics observed in N-terminal deletions ( $\Delta 2-17)$ (Terlau et al., 1997). In 
that report, the voltage tested lay in the moderate depolarization range, where $\mathrm{O}_{1}$ could be present, and $\Delta 2-17$ showed similar rectifying behavior to our N-terminal deletions. H343R restored the kinetics and features of the rectifying behavior, indicating a potential role for S4 in the rectifying behavior and the characteristic kinetics of the deletion mutants.

Among the possible mechanistic explanations of the biphasic behavior (diverging activation paths, inactivated states), we favor the hypothesis of the presence of two open states, which we named $\mathrm{O}_{1}$ and $\mathrm{O}_{2} . \mathrm{O}_{1}$ would be dominant at weak potentials, and $\mathrm{O}_{2}$ at strong potentials. Facilitated access to $\mathrm{O}_{1}$ would be the result of disturbing the PASCap-CNBHD interaction. As discussed above, the initial N-terminus (10-13) could stabilize a closed state when it interacts with S1 and CNBHD (Whicher and MacKinnon, 2019). We extend this surface of interaction to include the PASCap (2-25) domain. The PASCap-CNBHD may stabilize a closed state in the WT, limiting the access to $\mathrm{O}_{1}$ We refer to that surface as a "lock", since it stabilizes a closed state. The entire interaction surface of eag-CNBHD may also be critical to maintain the "lock" in position. In line with this view, deleting or mutating the intrinsic ligand showed a similar biphasic GV (Zhao et al., 2017). The intrinsic ligand plays a role in maintaining eag-CNBHD interaction, either allosterically (Codding and Trudeau, 2019) or directly (Haitin et al., 2013). 

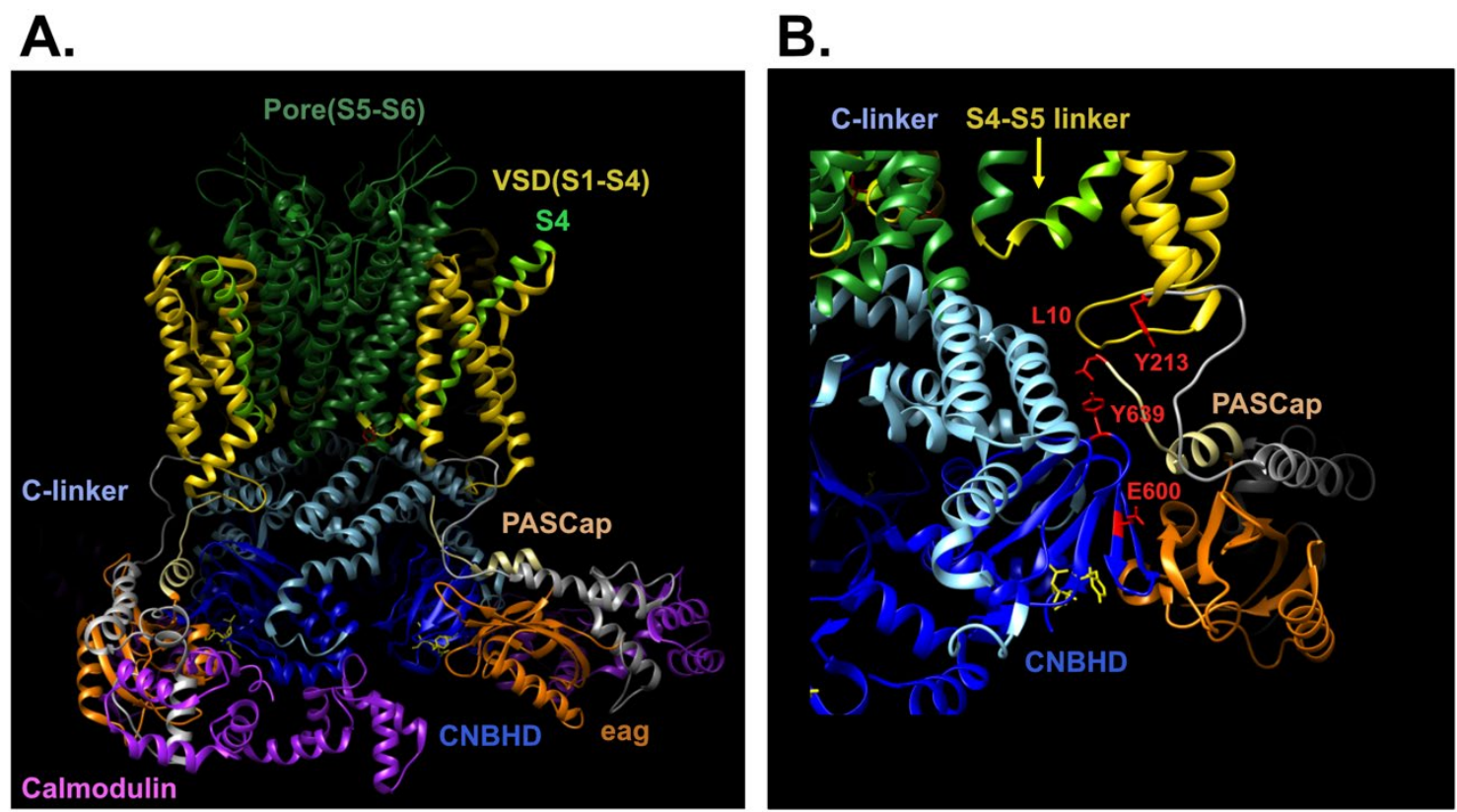

Figure 44. PASCap and CNBHD interaction surface that could stabilize a closed conformation. A) Cryo-EM structure of rat $\mathrm{K}_{v} 10.1$ (Whicher and MacKinnon, 2016). B) Enlarged view. The three residues identified by (Whicher and MacKinnon, 2019); L10 interacts with Y213 and Y639 to stabilize a closed conformation. E600R at the interface between PASCap and CNBHD.

The WT GV is monophasic and likely corresponds to $\mathrm{O}_{2}$. The activation kinetics supports this possibility. At strong potentials where $\mathrm{O}_{2}$ dominates, the mutant's kinetics became similar to WT activation kinetics.

\subsubsection{Different conductance of $\mathrm{O}_{1}$ and $\mathrm{O}_{2}$}

The currents of the mutants declined in amplitude with time during sustained depolarizing stimuli. $\Delta 2-10$ showed inactivation across all voltage ranges tested. A decrease in the test pulse amplitude appeared with pre-pulses of $-80 \mathrm{mV}$ and saturated at $+20 \mathrm{mV}$. This range of potential corresponds to the first component of the GV. This inactivating behavior was further confirmed with variable pre-pulse durations. The longer the pre-pulse, the less the current observed with the test potential. $\triangle$ PASCap inactivation appeared at moderate potentials, while E600R showed inactivation at moderate and strong potentials. This behavior could correspond to the conventional transition between a conducting and a non-conducting (inactivated) state. Still, it could also represent a transition from a state with larger conductance to another one with smaller (of which a non-conducting state would be an extreme case). Thus, we interpret this inactivation as a transition from $\mathrm{O}_{1}$ (a higher conductance state) to $\mathrm{O}_{2}$ (a 
state of lower conductance). The difference in conductance could also explain the rectifying behavior observed with the GV protocol. However, we cannot exclude the possibility of a third, entirely inactive state. The WT has been suggested to have intrinsic inactivation that can be amplified by 3-nitro-N-(4-phenoxyphenyl) benzamide (ICA105574) and a point mutation Y464A (Garg et al., 2012).

An argument in favor of two states with different conductances stems from our square-pulse experiments. In that protocol, the potential is driven repeatedly between two values corresponding to different regions of the GV, and the current obtained is therefore likely to reflect the different states of the channel. When we compared the current amplitude during a square pulse protocol where the voltage alternates between weak and strong potentials with the amplitude evoked by a constant pulse to the strong potential (where the channels are subject to the same depolarization for twice as long), the current amplitude was clearly larger in the square pulses ( $\Delta$ eag and E600R). A simple explanation would be that alternating the voltage limits the transition to $\mathrm{O}_{2}$ at strong potentials, which requires more prolonged depolarizations, and coexistence between $\mathrm{O}_{1}$ and $\mathrm{O}_{2}$ is maintained. The fraction of channels in $\mathrm{O}_{1}$ would then be more abundant than with the sustained depolarization. Therefore, the larger current amplitude suggests that $\mathrm{O}_{1}$ has a higher conductance than $\mathrm{O}_{2}$.

The increase in current amplitude during alternating voltage protocols was much smaller and limited to a narrow range of potentials for $\triangle$ PASCap and was almost non-existent for $\Delta 2-10$. This could mean that the conductance of $\mathrm{O}_{1}$ varies between the different mutants but can also be explained by different access rates to $\mathrm{O}_{1}$ (and therefore to $\mathrm{O}_{2}$ ), which could depend on the degree of distortion of the intracellular gating ring. Thus, $\mathrm{O}_{1}$ would be readily accessible for $\Delta$ eag and $\mathrm{E} 600 \mathrm{R}$, less accessible for $\triangle$ PASCap, and even less for $\Delta 2-10$.

As stated earlier, WT would have minimal access to $0_{1}$-Thus, we would predict that alternating protocols that reduce access to $\mathrm{O}_{2}$ would result in a smaller current amplitude. Consistent with this prediction, the current amplitude was smaller during alternating depolarizations than during a sustained pulse. 
$\Delta 2$-10. L341split provides another argument supporting that $\mathrm{O}_{1}$ has higher conductance than $\mathrm{O}_{2}$. This split channel loses both the first phase of the $\mathrm{GV}\left(\mathrm{O}_{1}\right)$ and the apparent inactivation observed with $\Delta 2-10$. Therefore, it is likely that the apparent inactivation reflects a transition from $\mathrm{O}_{1}$ to $\mathrm{O}_{2}$, which was compromised when $\mathrm{O}_{1}$ was lost.

\subsubsection{The biphasic behavior may correspond to two-voltage dependent gating events}

The biphasic behavior observed led us to propose a gating model for $\mathrm{K}_{\mathrm{v}} 10.1$, where activation occurs in two voltage-dependent gating events. First, the primary voltage sensor (S4) moves toward the extracellular side in response to depolarization. Then, the intracellular gating ring rotates to unwind the bundled helices at the bottom of S6 (the gate). Both steps would be coupled. The interaction between the cytoplasmic end of S4 (and interacting areas of the monomer) and the N-terminal tail would be responsible for transmitting the spatial changes of S4 to the inner gating ring. The first event, movement of the S4, may not be sufficient to open the gate at the bottom of S6 but would promote a conformation that can access $\mathrm{O}_{1}$, a transition with low probability if the inner gating ring is intact, like in WT. The N-terminus is proposed to act as a "lock", stabilizing a closed state in the WT channels (discussed above). We suggest that PASCap-CNBHD is an important interface that maintains that lock. Disrupting this interface (E600R and $\triangle$ PASCap, $\Delta 2-10$ ) increases the probability of populating an open state that we refer to as " $\mathrm{O}_{1}$ ". The mutants thus promote occupancy of $\mathrm{O}_{1}$. Facilitating access to $\mathrm{O}_{1}$ would be the common feature of the mutant phenotypes since a global fit fixing $\mathrm{O}_{1}$ components described the GV of all mutants (Fig. 24). In line with our model, $\mathrm{Mg}^{2+}$, which interferes with the movement of S4 (Schönherr et al., 2002), shifts $\mathrm{O}_{1}$ to depolarized potentials, leaving $\mathrm{O}_{2}$ unchanged (Fig. 25). Moreover, at least $\Delta 2-10$ requires a covalent link between S4 and S5 to display the biphasic behavior fully.

In L341split, where residues at the bottom of S4 and the S4-S5 linker are no longer connected to the voltage sensor's movement, remarkably attenuated the first component of the $\mathrm{GV}\left(\mathrm{O}_{1}\right)$. The coupling of the movement sensor to the gate is likely interrupted in the split channel. It is also plausible that the bottom of the S4 interacts directly with the channel gate. The $\mathrm{C}$-linker is a good candidate for such interaction as 
suggested by the structure (Whicher and MacKinnon, 2016). However, the intracellular gating ring, including the C-linker, is dispensable for voltage-dependent gating. Deleting the intracellular domains did not abolish voltage-dependent gating (Whicher and MacKinnon, 2019), suggesting that the sensor may be directly coupled to the gate (through the bottom of S4 or the S4-S5 linker). The sensor's movement can be positively or negatively coupled to the gate; the sensor may actively open the gate (positive coupling), but it also might keep the gate closed at rest, and its removal allows the opening of the gate. It is out of our scope to discuss it in detail, but recent reports suggest that the pore prefers a closed conformation (Tomczak et al., 2017; Whicher and MacKinnon, 2019). Therefore, the movement of the S4 could "open the channel gate".

The rotation of the gating ring would be the second gating event. Comparison of cryo-EM structures of hERG and rat Kv10.1 revealed that the ring of Kv10.1, which has its voltage sensors in the activated position but the gate closed because of interaction with $\mathrm{Ca}^{2+}$-Calmodulin, is rotated counterclockwise (seen from the extracellular side) with respect to HERG, which is open. This rotation would also be voltage-dependent, either intrinsically or because the permissive movement of the VSD is voltagedependent. Voltage-sensitive rearrangements in Elk channels' intracellular ring have been reported (Dai and Zagotta, 2017). The N-terminus is a potential candidate for coupling the voltage sensor to the ring movement. It has been proposed that the bottom of S4 interacts with the initial N-terminus to stabilize an open state (Tomczak, 2016) (Whicher and MacKinnon, 2019). The mutation D342C likely interferes with this coupling step (Fig39). Fitting the biphasic GV of D342C showed a shift of the second gating component to the right. Introducing a Cys residue at this critical location probably underlies the biphasic behavior. A series of point mutations at D342 showed mostly a shift of the GV to the right while maintaining a monophasic response (Tomczak, 2016). Intriguingly, introducing a Cys mutation at Y344 showed a rectifying behavior, absent in Y344A or Y344F (Lin et al., 2014). The cause is unclear, but it is plausible that a Cys at the bottom of S4 (D342) or the beginning of S4-S5 (Y344) decouples the gating events. Moreover, the hydroxyl group in Y347split (interrupted after Y347residue) could also play a role in coupling the two events in the split channel. 
Y347F and Y347L showed a biphasic behavior similar to D342C, and the second component is shifted to the right (Tomczak, 2016).

In summary, we propose a model (Fig.45), where the voltage sensor can occupy two positions, "Resting"or "Active". The transition to "Active" would allow the corresponding inner gating ring component to move from "Closed" to "Open". When all four VSDs are in the "Active" conformation $\left(\mathrm{A}^{4} \mathrm{C}^{4}\right)$, the channel gate (whether it is the already described Gln476 or not) can open, giving access to $\mathrm{O}_{1}$. In the presence of the correctly positioned and composed inner ring, this transition is possible, albeit unlikely. Distortion of the inner ring increases the probability for occupancy of $\mathrm{O}_{1}$. When all four subunits of the ring are in the "Open" position $\left(\mathrm{A}^{4} \mathrm{C}^{0}\right)$, access to the stable (but lower conductance) state $\mathrm{O}_{2}$ is possible.

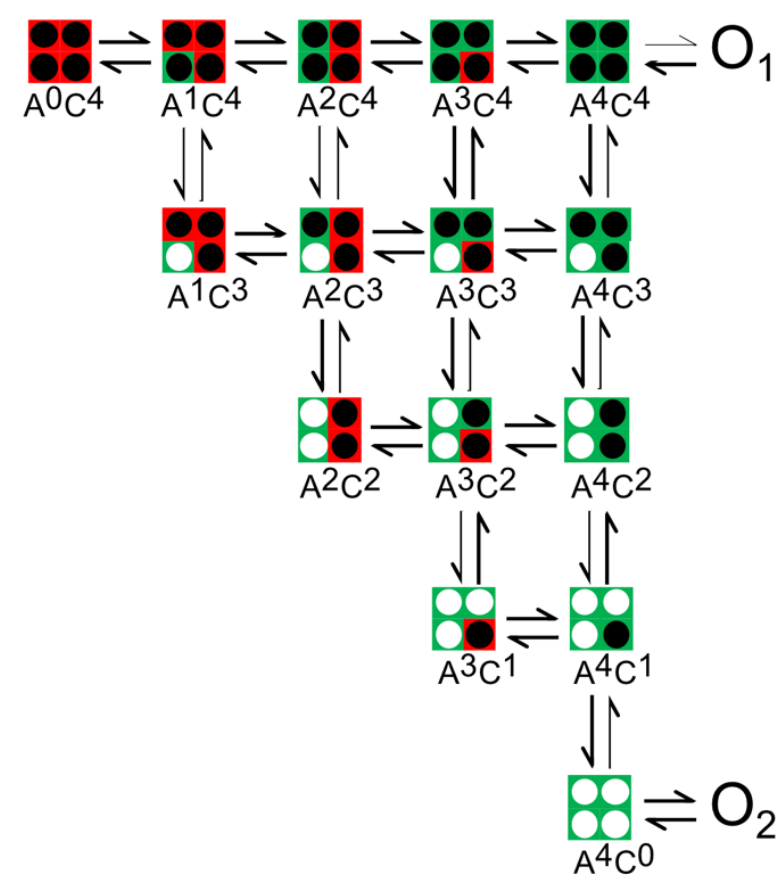

Figure 45. Proposed model for Kv10.1 gating Red and green represent the position of the sensor resting or active, respectively. Filled and empty circles represent the ring conformation closed and open, respectively. 


\subsection{Calmodulin may contribute to $\mathrm{K}_{\mathrm{v}} 10.1$ gating machinery at basal $\mathrm{Ca}^{2+}$ level}

\subsubsection{The C-lobe of Calmodulin binds Kv10.1 at basal Ca ${ }^{2+}$ levels}

Our data suggest that Calmodulin is bound to $\mathrm{K}_{\mathrm{v}} 10.1$ at the physiological intracellular concentration of $\mathrm{Ca}^{2+}(100 \mathrm{nM}$ (Cork et al., 1987)). In Xenopus oocyte and HEK293 cell extracts, Myc-tagged Calmodulin pulled down $K_{v} 10.1$ in the presence of $\mathrm{Ca}^{2+}(100 \mathrm{nM})$. This observation is in agreement with previous studies that reported $\mathrm{Ca}^{2+}$ dependent binding of Calmodulin (Schönherr et al., 2000; Ziechner et al., 2006). The pull-down suggests that $100 \mathrm{nM} \mathrm{Ca}^{2+}$ is sufficient for saturating Calmodulin binding to $\mathrm{K}_{\mathrm{v}}$ 10.1. A previous study that tested the binding of Calmodulin to C-terminal fragments of Kv10.1 using a pull-down assay reported a faint band at $100 \mathrm{nM} \mathrm{Ca}^{2+}$ (Schönherr et al., 2000). Another study reported weak binding of Calmodulin to BD-C2, the C-terminal binding domain, even in the absence of $\mathrm{Ca}^{2+}$. This weak binding was observed only if the full-length channel was used (Ziechner et al., 2006). Therefore, we propose that the intracellular concentration of Xenopus laevis oocytes, which is reported to be around $100 \mathrm{nM}(92.6 \pm 30 \mathrm{nM}$ at resting membrane potential) (Cork et al., 1987), is sufficient for Calmodulin binding. We propose that only the C-lobe of Calmodulin, which has a high affinity for $\mathrm{Ca}^{2+}(100 \mathrm{nM}$ (James et al., 1995)) is bound to $\mathrm{K}_{\mathrm{v}} 10.1$ at basal $\mathrm{Ca}^{2+}$ concentrations, while the $\mathrm{N}$-lobe (Kd $=1 \mu \mathrm{M}$ (James et al., 1995)) binds when $\mathrm{Ca}^{2+}$ concentration rises (Fig. 46). $\mathrm{Ca}^{2+}$ would then occupy the C-lobe (EF $3,4)$ EF-hands at resting levels, while the EF-hands of the N-lobe $(\mathrm{EF} 1,2)$ would be $\mathrm{Ca}^{2+}$ free. Indeed, a crystal structure of Calmodulin bound to BD-C2 of $\mathrm{K}_{\mathrm{v}} 10.1$ reports a conformation of $\mathrm{Ca}^{2+}$ bound $\mathrm{C}$-lobe and a $\mathrm{Ca}^{2+}$ free N-lobe (Marques-Carvalho et al., 2016), further supporting our proposal. 

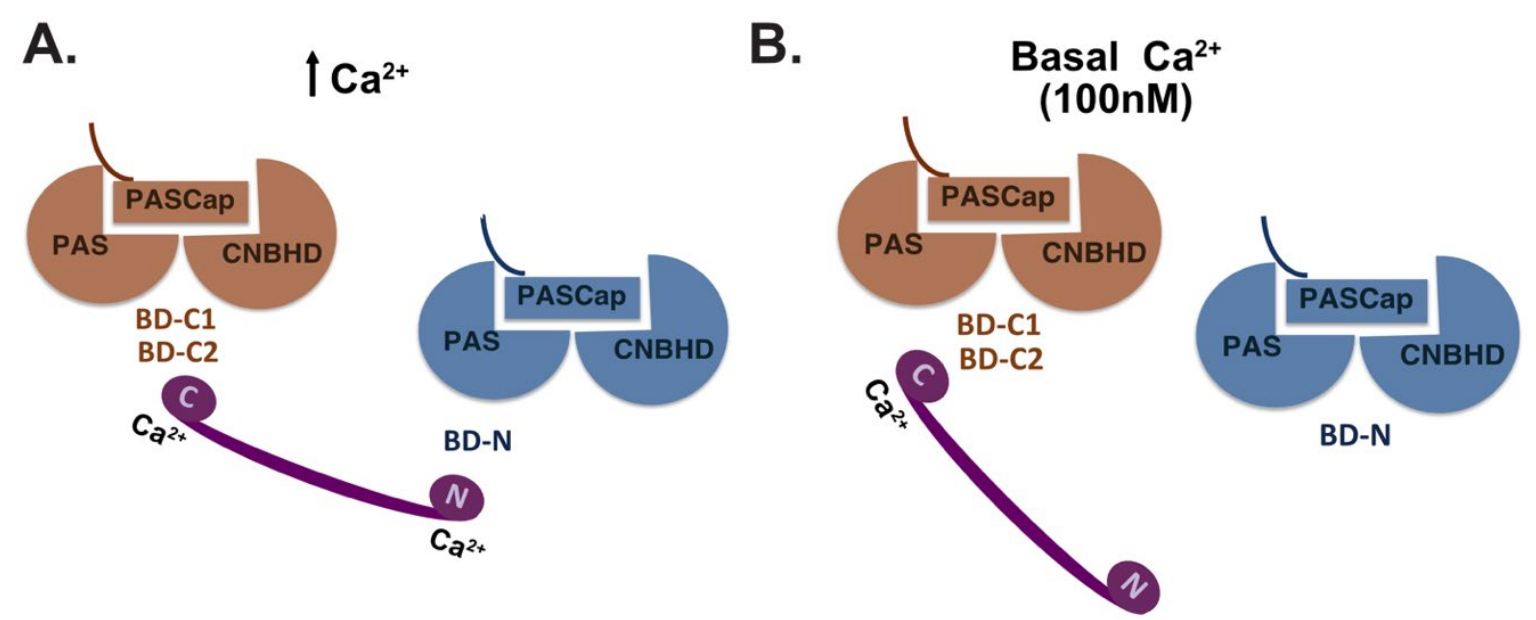

Figure 46. The C-lobe of calmodulin may bind to BD-C2 at basal Ca2+ level. A) Cartoon depicting intracellular domains of opposing subunits. $\mathrm{Ca}^{2+}$ binds to both lobes of calmodulin ( $\mathrm{N}-$ and $\mathrm{C}$-lobe) when intracellular $\mathrm{Ca}^{2+}$ is raised. Calmodulin crossbridges opposite subunit, it could act as a clamp B) Cartoon depicting intracellular domains of opposing subunits. At basal $\mathrm{Ca}^{2+}$, only C-lobe is bound to $\mathrm{Ca}^{2+}$. C-lobe is bound to c-terminal binding domain (BD-C2).

\subsection{2 $\mathrm{Ca}^{2+}$ Calmodulin inhibits $\mathrm{K}_{\mathrm{v}} 10.1$ through the intracellular ring}

Calmodulin binds to three bindings sites: two sites at the C-terminus (BD-C1, BD-C2) and one at the N-terminus (BD-N). The N-terminal binding sites are located after the eag domain, while the C-terminal binding sites are found after the CNBHD (Schönherr et al., 2000; Whicher and MacKinnon, 2016; Ziechner et al., 2006). The $\mathrm{Ca}^{2+-}$ bound C-lobe exposes hydrophobic patches that bind to BD-C2 (Marques-Carvalho et al., 2016; Whicher and MacKinnon, 2016). The C-lobe also interacts with BD-C1, but this interaction is less critical for Calmodulin binding (Goncalves and Stuhmer, 2010; Ziechner et al., 2006). However, mutation of BD-C1 impairs inhibition by Calmodulin (Ziechner et al., 2006) and interfere with PAS-CNBHD binding. This site of interaction should not be overlooked. The N-lobe of Calmodulin binds to BD-N of an opposing subunit (Whicher and MacKinnon, 2016). $\mathrm{Ca}^{2+}$ Calmodulin cross-bridging opposite subunits and stabilizing a closed conformation, as captured by the Cryo-EM, suggested a global change in the ring. In this model, $\mathrm{Ca}^{2+}$-Calmodulin may act as a "clamp" that interferes with the ring rotation. A counterclockwise rotation of the ring (seen from the extracellular view) seems to be correlated to the gate opening (James and Zagotta, 2018; Wang and MacKinnon, 2017; Whicher and MacKinnon, 2016). Intriguingly, a 
Cryo-EM structure of $\Delta 2-13$ bound to $\mathrm{Ca}^{2+}$ Calmodulin undergo a partial rotation compared to the open conformation of hERG (Wang and MacKinnon, 2017; Whicher and MacKinnon, 2019). This conformation would represent a pre-open state (Whicher and MacKinnon, 2019). This partial rotation could support the clamp model, where Calmodulin traps the ring and hinders its rotation. In this model, the binding of Calmodulin to both N- and C-terminal sites would be crucial for current inhibition.

However, functional data suggest that the C-lobe binding to BD-C2 is sufficient, although less efficient, to induce inhibition, since a mutant Calmodulin with $\mathrm{Ca}^{2+}$ insensitive N-lobe still inhibits $\mathrm{K}_{\mathrm{v}} 10.1$, albeit with reduced efficiency (Ziechner et al., 2006). Impairing Calmodulin binding to BD-N showed a current reduction, although it required longer time (Lorinczi et al., 2016). These reports suggest that local conformational changes at BD-C2 instead of a clamp model may mediate inhibition. It is plausible that the binding of Calmodulin at BD-C2 influences the interaction of eag and CNBHD domains. The binding of the C-lobe at BD-C2 could allow it to interact with BD-C1, which has been reported to influence eag-CNBHD interaction. Moreover, the Cryo-EM structure shows a third interface of interaction that had not been reported as a binding site for Calmodulin (Whicher and MacKinnon, 2016). This interface lies within the CNBHD before the intrinsic ligand, but on a different subunit. It expands the possible interaction network of Calmodulin with the intracellular domains. (Fig. 47). 

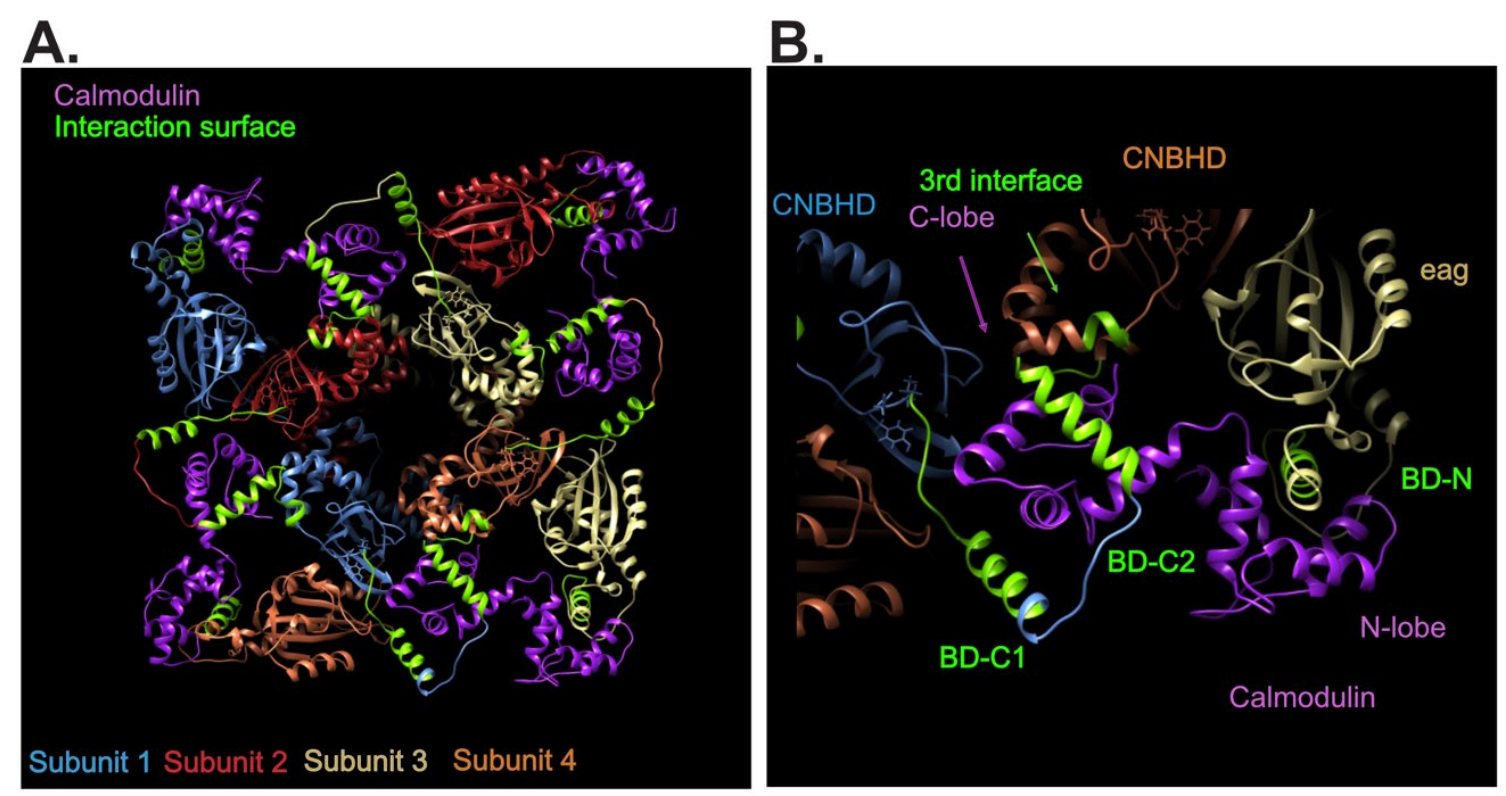

Figure 47. Calmodulin interacts with three subunits. Rat $\mathrm{K}_{\mathrm{v}} 10.1$ Cryo-EM structure (Whicher and MacKinnon, 2016). A) Bottom view. Each subunit is indicated with a different color. Calmodulin is in purple. Interaction surface is in green B) One calmodulin interacts with three subunits. C-terminal binding sites (BDC-1 and BDC-2) belong to subunit 1 . N-terminal binding site (BD-N) belongs to subunit3. C-lobe of calmodulin interacts with a $3^{\text {rd }}$ subunit ( $3^{\text {rd }}$ interface) which belongs to subunit4.

It seems likely that the C-lobe of Calmodulin influences the eag-CNBHD interaction and consequently the gating of the channel. In this scenario, the binding at the $\mathrm{N}$-terminus is not required but rather enhances the efficiency of inhibition. This model poses a relevant question. If the binding of Calmodulin at BD-C2 suffices to mediate inhibition, how could Calmodulin be bound at basal $\mathrm{Ca}^{2+}$ level as we propose? The difference could lie in the number of Calmodulin molecules bound per channel. As mentioned above, the binding of the C-lobe-lobe to $\mathrm{Ca}^{2+}$ has a $\mathrm{Kd}$ of $100 \mathrm{nM}$ (James et al., 1995), which is close to the intracellular level of Ca ${ }^{2+}$ (Cork et al., 1987). Moreover, Calmodulin was reported to bind to a fragment containing BDC2 with a Kd of 100150nM (Ziechner et al., 2006)

In summary, two models have been proposed for inhibition by $\mathrm{Ca}^{2+}$ a) A clamp model that causes a global change b) Local conformational changes close to BD-C2 (Marques-Carvalho et al., 2016). It seems likely that both mediate $\mathrm{Ca}^{2+}$ Calmodulin inhibition. In both models, Calmodulin inhibits $\mathrm{K}_{\mathrm{v}} 10.1$ through the intracellular domains as reported by Lörinczi,E. et.al. , 2016. This idea is not so foreign; Calmodulin 
has been reported to inhibit $C N G$ channels, close relatives to $K C N H$, through disrupting the interaction between N- \& C-terminal regions (Trudeau and Zagotta, 2003). Both models also highlight the binding of the C-lobe of Calmodulin to BD-C2 as a critical step for binding and inhibiting the channel. Indeed, in our hands, mutations at BD-C2 (F714SF717S) had more significant impact on the N-terminal deletions than mutations at BD-N (F151NL154N).

\subsection{3 $\mathrm{Ca}^{2+}$ Calmodulin could stabilize $\mathrm{O}_{1}$}

Our data shows that the Calmodulin binding site mutants $\Delta 2-10 .^{\mathrm{F} 714 S F 717 \mathrm{~S}}$ and

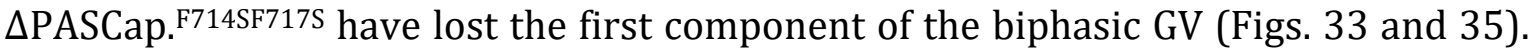
$\triangle$ PASCap F714SF717S has also lost the current potentiation observed at $+40 \mathrm{mV}$ when preceded by hyperpolarizing pre-pulses (Fig. 37). On the other hand, $\Delta 2-10^{\mathrm{F} 151 \mathrm{NL} 154 \mathrm{~N}}$ and $\triangle$ PASCap. ${ }^{\text {F151NL154N }}$ retained the biphasic GV. $\triangle$ PASCap ${ }^{\text {F151NL154N }}$ shows attenuated current potentiation in response to hyperpolarizing pre-pulses. F714SF717S and F151NL154N disrupt binding of Calmodulin to Kv10.1 (Schönherr et al., 2000; Ziechner et al., 2006).

In line with the arguments discussed above, we propose that, at basal $\mathrm{Ca}^{2+}$ level, Calmodulin is bound at BD-C2, but not at BD-N. The binding stabilizes the first component of the mutants' GV, which corresponds to $\mathrm{O}_{1}$, the component that could be stabilized by hyperpolarizing pre-potentials. We, therefore, hypothesize that Calmodulin stabilizes $\mathrm{O}_{1}$. In line with this hypothesis, $\triangle$ PASCap and E600R showed a linear monophasic IV in response to an increase of $\mathrm{Ca}^{2+}$. The current at moderate potentials, where $0_{1}$ is proposed to prevail, was sustained for a longer time than the current at strong potentials, where $\mathrm{O}_{2}$ would dominate. In addition to the change in IV, the current was remarkably potentiated as previously reported by Lörinczi,E. et.al. ,2016. This observation is in line with our proposal that $\mathrm{O}_{1}$ could have higher conductance than $\mathrm{O}_{2}$. It could also explain the current observed at negative potentials in $\Delta 2-13$ (Whicher and MacKinnon, 2019).

We propose that, in high $\mathrm{Ca}^{2+}$, Calmodulin interferes with the rotation of the gating ring by hindering the interaction between eag and CNBHD and form a clamp 
between ring subunits when $\mathrm{Ca}^{2+}$ raises. Therefore, calmodulin would drive the channel population towards the first line in the model (AC, AC, AC). In WT, these states are improbable to have access to $\mathrm{O}_{1}$. This results in current inhibition as access to $\mathrm{O}_{2}$ is hindered. However, the mutants distort an interaction between PASCap and CNBHD, which might allow a partial rotation of the ring as reported by (Whicher and MacKinnon, 2019) and consequently access $0_{1}$, leading to current potentiation rather than inhibition because of the larger conductance of $\mathrm{O}_{1}$.

The degree of distortion of the gating ring by the mutants also differs. $\Delta 2-10$ affects only one residue in the surface discussed above (Fig. 44) (Whicher and MacKinnon, 2019). Therefore, it is not surprising that compared to other mutants, it is the closest one to the WT. Square pulses did not induce current recovery, and Calmodulin was able to cause initial inhibition. However, the larger the distortion in the ring, the easier the access to $\mathrm{O}_{1}$. Alternatively, the conductance of $\mathrm{O}_{1}$ could be different across mutants.

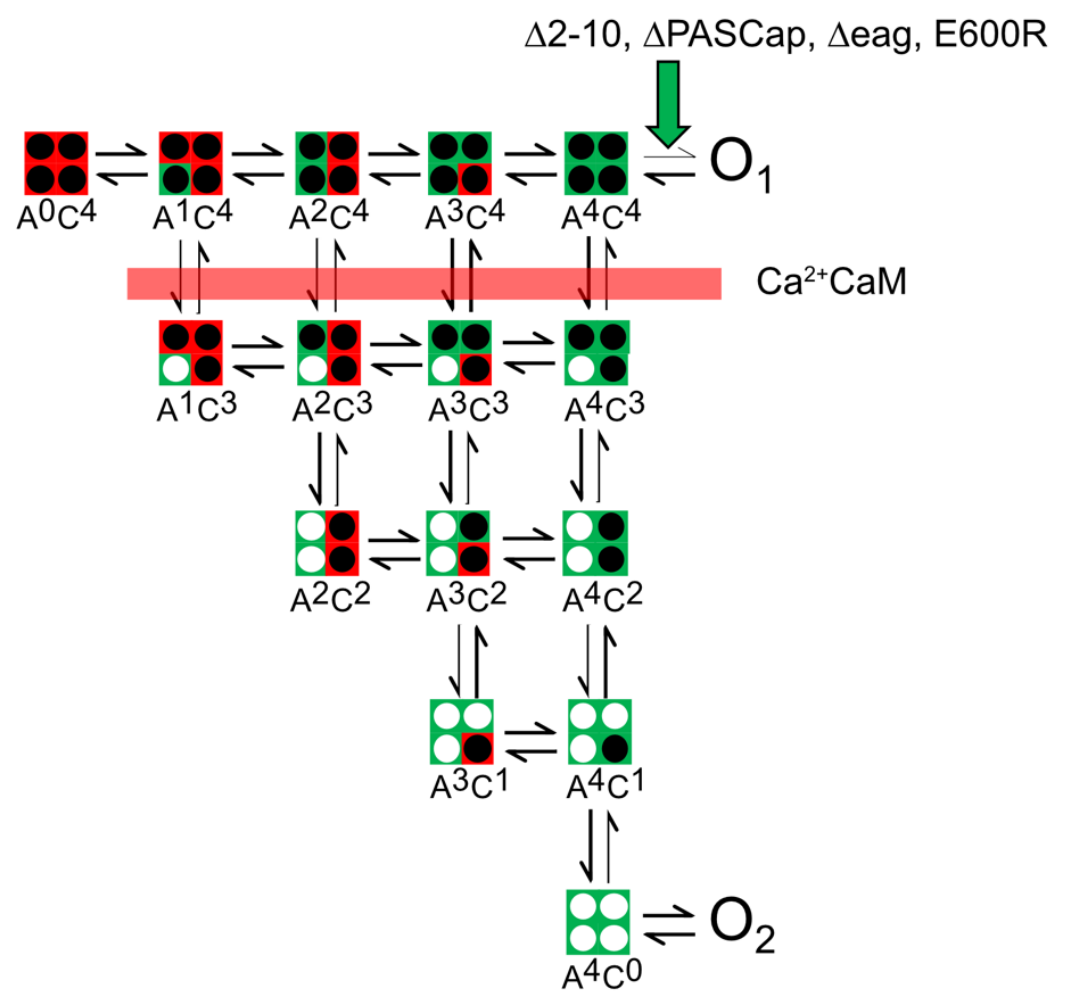

Figure 48. Proposed model for Ca2+ Calmodulin inhibition. Red and green represent the position of the sensor resting or active, respectively. Filled and empty circles represent the ring conformation closed and open, respectively. $\mathrm{Ca}^{2+} \mathrm{CaM}^{\mathrm{M}}$ inhibition shown as a red line. 


\subsection{Role of intracellular domains in the gating of Kv10.1}

We have focused our attention on the $\mathrm{N}$-terminal deletions because the peculiar biphasic behavior offers a path to study the inner gating ring's role. However, to have an overview of the role of the complete ring in gating, it is pertinent to discuss the Cterminal domain (CNBHD) as well. We performed a deletion of the CNBHD, which resulted in a shift of the GV to the right and a loss of the Cole-Moore effect. A similar observation was already reported by (Zhao et al., 2017). The rightward shift of the GV indicates that stronger depolarizations are required to open the channel. Intriguingly, deleting all intracellular domains resulted in a similar effect; rightward shift of the GV and a loss of Cole-Moore (Whicher and MacKinnon, 2019). The presence of the ring likely plays a role in stabilizing an open state (Robertson and Morais-Cabral, 2020; Zhao et al., 2017). The C-linker and CNBHD seem to be critical molecular players for this role.

CNBHD is similar to CNBD. Cyclic nucleotides bind to CNBD and mediate the gating of CNG channels. cAMP binds to the CNBD of HCN and counteracts the inhibitory role of CNBD (Porro et al., 2019). It is plausible that CNBHD performs a similar function in KCNH channels.

However, CNBHD does not bind to cyclic nucleotides. Instead, the pocket is occupied by the intrinsic ligand. The ligand would stabilize an open state across $K C N H$ family members (Robertson and Morais-Cabral, 2020). The ligand has been reported to lie on the surface of interaction between eag and CNBHD (Haitin et al., 2013). The eag domain, which characterizes the KCNH family, sets it apart from CNG and HCN channels. It may have evolved to provide a modulatory domain that modulates the CNBHD. We propose that it acts as a "lock" and stabilizes a non-conducting state. The movement of the sensor removes the lock and probably allows/pulls the channel open. Removal of the lock may allow/trigger the rotation of the ring. The two events: movement of the sensor and the ring rotation are tightly coupled in WT. Distorting the ring with $\mathrm{N}$-terminal deletions decouple the two events and reveals an open state: " $\mathrm{O}_{1}$ ". 


\subsection{Outlook}

Our work provides insights into a set of interactions that play a role in the gating of $\mathrm{K}_{\mathrm{v}}$ 10.1. We have shed light on the N-terminus' role in coupling the movement of the sensor to a second gating event, possibly the rotation of the intracellular gating ring. We propose a model to explain the two gating transitions. In the future, we plan to challenge the model experimentally. We point out a role for Calmodulin in the gating of $\mathrm{K}_{\mathrm{v}}$ 10.1. Our data suggest that Calmodulin is bound to $\mathrm{K}_{\mathrm{v}} 10.1$ at rest and participates in gating, and it stabilizes $\mathrm{O}_{1}$, with elevated intracellular $\mathrm{Ca}^{2+}$, the open state revealed by the $\mathrm{N}$-terminal deletions. The low probability of occupancy of $\mathrm{O}_{1}$ in the presence of a properly formed inner gating ring (WT), explains the inhibition of the current by $\mathrm{Ca}^{2+}$ Calmodulin. In the future, we plan to improve the pull-down assay and titrate $\mathrm{Ca}^{2+}$ concentrations required for binding, as well as determining the relevance of each lobe using Calmodulin mutants (already available in the lab). We also plan to test the binding of Calmodulin to the N-terminal deletion mutants.

Despite the progress made in investigating the electromechanical coupling of $\mathrm{K}_{v} 10.1$, there are still open questions. The movement of the sensor is coupled to the inner gating ring through different sets of interactions. We have discussed one of those sets, but more work is required to comprehend the gating machinery fully. Other open questions in the field concern the assembly of the ring. It is not clear yet whether the ring is always assembled or if the pocket is always occupied with the intrinsic ligand (Robertson and Morais-Cabral, 2020). The structural data has been quite insightful. However, they provided us with snapshots of limited conformations of the channel. Functional studies are crucial to draw a clearer picture. 


\section{References}

Aggarwal, S.K., and MacKinnon, R. (1996). Contribution of the S4 segment to gating charge in the Shaker K+ channel. Neuron 16, 1169-1177.

Badaoui, M., Mimsy-Julienne, C., Saby, C., Van Gulick, L., Peretti, M., Jeannesson, P., Morjani, H., and Ouadid-Ahidouch, H. (2018). Collagen type 1 promotes survival of human breast cancer cells by overexpressing Kv10.1 potassium and Orai1 calcium channels through DDR1-dependent pathway. Oncotarget 9, 24653-24671.

Bauer, C.K., and Schwarz, J.R. (2018). Ether-a-go-go $K(+)$ channels: effective modulators of neuronal excitability. J Physiol 596, 769-783.

Bayley, P.M., Findlay, W.A., and Martin, S.R. (1996). Target recognition by calmodulin: dissecting the kinetics and affinity of interaction using short peptide sequences. Protein Sci 5, 1215-1228.

Brelidze, T.I., Carlson, A.E., and Zagotta, W.N. (2009). Absence of direct cyclic nucleotide modulation of mEAG1 and hERG1 channels revealed with fluorescence and electrophysiological methods. J Biol Chem 284, 27989-27997.

Bruggemann, A., Pardo, L.A., Stuhmer, W., and Pongs, O. (1993). Ether-a-go-go encodes a voltage-gated channel permeable to $\mathrm{K}+$ and $\mathrm{Ca} 2+$ and modulated by cAMP. Nature $365,445-448$.

Bruggemann, A., Stuhmer, W., and Pardo, L.A. (1997). Mitosis-promoting factormediated suppression of a cloned delayed rectifier potassium channel expressed in Xenopus oocytes. Proc Natl Acad Sci U S A 94, 537-542.

Carlson, A.E., Brelidze, T.I., and Zagotta, W.N. (2013). Flavonoid regulation of EAG1 channels. J Gen Physiol 141, 347-358. 
Cázares-Ordoñez, V., and Pardo, L.A. (2015). Kv10.1 potassium channel: from the brain to the tumors. $20,1-31$.

Chaney, M., and Steinrauf, L. (1974). The crystal and molecular structure of tetragonal L-cystine. Acta Crystallographica Section B Structural Crystallography and Crystal Chemistry 30, 711-716.

Chen, H., Chatelain, F.C., and Lesage, F. (2014). Altered and dynamic ion selectivity of $\mathrm{K}+$ channels in cell development and excitability. Trends Pharmacol Sci 35, 461-469.

Codding, S.J., Johnson, A.A., and Trudeau, M.C. (2020). Gating and regulation of KCNH (ERG, EAG, and ELK) channels by intracellular domains. Channels (Austin) 14, 294-309.

Codding, S.J., and Trudeau, M.C. (2019). The hERG potassium channel intrinsic ligand regulates N- and C-terminal interactions and channel closure. J Gen Physiol 151, 478488.

Cole, K.S., and Moore, J.W. (1960). Potassium Ion Current in the Squid Giant Axon: Dynamic Characteristic. Biophysical Journal 1, 1-14.

Cork, R.J., Cicirelli, M.F., and Robinson, K.R. (1987). A rise in cytosolic calcium is not necessary for maturation of Xenopus laevis oocytes. Developmental Biology 121, 4147.

Dai, G., James, Z.M., and Zagotta, W.N. (2018). Dynamic rearrangement of the intrinsic ligand regulates KCNH potassium channels. J Gen Physiol 150, 625-635.

Dai, G., and Zagotta, W.N. (2017). Molecular mechanism of voltage-dependent potentiation of KCNH potassium channels. Elife 6.

de la Peña, P., Alonso-Ron, C., Machín, A., Fernández-Trillo, J., Carretero, L., Domínguez, P., and Barros, F. (2011). Demonstration of physical proximity between the $\mathrm{N}$ terminus 
and the S4-S5 linker of the human ether-a-go-go-related gene (hERG) potassium channel. J Biol Chem 286, 19065-19075.

de la Peña, P., Domínguez, P., and Barros, F. (2018). Functional characterization of Kv11.1 (hERG) potassium channels split in the voltage-sensing domain. Pflügers Archiv - European Journal of Physiology 470, 1069-1085.

de la Pena, P., Machin, A., Fernandez-Trillo, J., Dominguez, P., and Barros, F. (2015). Interactions between the $\mathrm{N}$-terminal tail and the gating machinery of hERG $\mathrm{K}(+)$ channels both in closed and open/inactive states. Pflugers Arch 467, 1747-1756.

de la Peña, P., Machín, A., Fernández-Trillo, J., Domínguez, P., and Barros, F. (2015). Interactions between the $\mathrm{N}$-terminal tail and the gating machinery of hERG $\mathrm{K}+$ channels both in closed and open/inactive states. Pflugers Archiv European Journal of Physiology 467, 1747-1756.

del Camino, D., Holmgren, M., Liu, Y., and Yellen, G. (2000). Blocker protection in the pore of a voltage-gated $\mathrm{K}+$ channel and its structural implications. Nature 403, 321325.

Destexhe, A., and Huguenard, J.R. (2010). Modeling voltage-dependent channels. In Computational Modeling Methods for Neuroscientists, S. Erik De, ed. (The MIT Press), pp. 107-137.

Eyring, H. (1935). The Activated Complex in Chemical Reactions. The Journal of Chemical Physics 3, 107-115.

Garg, V., Sachse, F.B., and Sanguinetti, M.C. (2012). Tuning of EAG K(+) channel inactivation: molecular determinants of amplification by mutations and a small molecule. J Gen Physiol 140, 307-324.

Goncalves, J.T., and Stuhmer, W. (2010). Calmodulin interaction with hEAG1 visualized by FRET microscopy. PLoS One 5, e10873. 
Gonzalez, C., Baez-Nieto, D., Valencia, I., Oyarzun, I., Rojas, P., Naranjo, D., and Latorre, R. (2012). K(+) channels: function-structural overview. Compr Physiol 2, 2087-2149.

Goodchild, S.J., Macdonald, L.C., and Fedida, D. (2015). Sequence of gating charge movement and pore gating in HERG activation and deactivation pathways. Biophys J $108,1435-1447$.

Gustina, A.S., and Trudeau, M.C. (2011). hERG potassium channel gating is mediated by $\mathrm{N}$ - and C-terminal region interactions. J Gen Physiol 137, 315-325.

Gustina, A.S., and Trudeau, M.C. (2012). HERG potassium channel regulation by the Nterminal eag domain. Cell Signal 24,1592-1598.

Gutman, G.A., Chandy, K.G., Grissmer, S., Lazdunski, M., McKinnon, D., Pardo, L.A., Robertson, G.A., Rudy, B., Sanguinetti, M.C., Stuhmer, W., et al. (2005). International Union of Pharmacology. LIII. Nomenclature and molecular relationships of voltagegated potassium channels. Pharmacol Rev 57, 473-508.

Haitin, Y., Carlson, A.E., and Zagotta, W.N. (2013). The structural mechanism of KCNHchannel regulation by the eag domain. Nature 501, 444-448.

Han, B., He, K., Cai, C., Tang, Y., Yang, L., Heinemann, S.H., Hoshi, T., and Hou, S. (2016). Human EAG channels are directly modulated by PIP2 as revealed by electrophysiological and optical interference investigations. Scientific Reports 6, 23417-23417.

Han, B., Tokay, T., Zhang, G., Sun, P., and Hou, S. (2017). Eag1 K(+) Channel: Endogenous Regulation and Functions in Nervous System. Oxid Med Cell Longev 2017, 7371010.

Hernandez-Resendiz, I., Hartung, F., and Pardo, L.A. (2019). Antibodies Targeting K V Potassium Channels: A Promising Treatment for Cancer. Bioelectricity 1, 180-187. 
Herrmann, S., Ninkovic, M., Kohl, T., Lörinczi, É., and Pardo, L.A. (2012). Cortactin controls surface expression of the voltage-gated potassium channel $K(V) 10.1$. The Journal of biological chemistry 287, 44151-44163.

Hille, B. (2007). Ion channels of excitable membranes (Sunderland, Mass.: Sinauer). Hodgkin, A.L., and Huxley, A.F. (1952a). The components of membrane conductance in the giant axon of Loligo. J Physiol 116, 473-496.

Hodgkin, A.L., and Huxley, A.F. (1952b). Currents carried by sodium and potassium ions through the membrane of the giant axon of Loligo. J Physiol 116, 449-472.

Hoshi, T., and Armstrong, C.M. (2015). The Cole-Moore Effect: Still Unexplained? Biophys J 109, 1312-1316.

Imbrici, P., Grottesi, A., D'Adamo, M.C., Mannucci, R., Tucker, S.J., and Pessia, M. (2009). Contribution of the central hydrophobic residue in the PXP motif of voltage-dependent $\mathrm{K}+$ channels to S6 flexibility and gating properties. Channels (Austin) 3, 39-45.

James, P., Vorherr, T., and Carafoli, E. (1995). Calmodulin-binding domains: just two faced or multi-faceted? Trends Biochem Sci 20, 38-42.

James, Z.M., and Zagotta, W.N. (2018). Structural insights into the mechanisms of CNBD channel function. J Gen Physiol 150, 225-244.

Jenke, M., Sanchez, A., Monje, F., Stuhmer, W., Weseloh, R.M., and Pardo, L.A. (2003). Cterminal domains implicated in the functional surface expression of potassium channels. EMBO J 22, 395-403.

Kaplan, W.D., and Trout, W.E. (1969). THE BEHAVIOR OF FOUR NEUROLOGICAL MUTANTS OF DROSOPHILA. Genetics 61, 399. 
Kawasaki, H., Soma, N., and Kretsinger, R.H. (2019). Molecular Dynamics Study of the Changes in Conformation of Calmodulin with Calcium Binding and/or Target Recognition. Sci Rep 9, 10688.

Komeiji, Y., Ueno, Y., and Uebayasi, M. (2002). Molecular dynamics simulations revealed $\mathrm{Ca}(2+)$-dependent conformational change of Calmodulin. FEBS Lett 521, 133 139.

Kopec, W., Kopfer, D.A., Vickery, O.N., Bondarenko, A.S., Jansen, T.L.C., de Groot, B.L., and Zachariae, U. (2018). Direct knock-on of desolvated ions governs strict ion selectivity in $\mathrm{K}(+)$ channels. Nat Chem 10, 813-820.

Krishnan, M.N., Bingham, J.P., Lee, S.H., Trombley, P., and Moczydlowski, E. (2005). Functional role and affinity of inorganic cations in stabilizing the tetrameric structure of the KcsA K+ channel. J Gen Physiol 126, 271-283.

Kuang, Q., Purhonen, P., and Hebert, H. (2015). Structure of potassium channels. Cell Mol Life Sci 72, 3677-3693.

Lin, T.F., Jow, G.M., Fang, H.Y., Fu, S.J., Wu, H.H., Chiu, M.M., and Jeng, C.J. (2014). The Eag domain regulates the voltage-dependent inactivation of rat Eag1 $\mathrm{K}+$ channels. PLoS One 9, e110423.

Linaro, D., and Giugliano, M. (2015). Markov Models of Ion Channels. In Encyclopedia of Computational Neuroscience, D. Jaeger, and R. Jung, eds. (New York, NY: Springer New York), pp. 1649-1660.

Lockless, S.W., Zhou, M., and MacKinnon, R. (2007). Structural and thermodynamic properties of selective ion binding in a K+ channel. PLoS Biol 5, e121. 
Lorinczi, E., Gomez-Posada, J.C., de la Pena, P., Tomczak, A.P., Fernandez-Trillo, J., Leipscher, U., Stuhmer, W., Barros, F., and Pardo, L.A. (2015). Voltage-dependent gating of KCNH potassium channels lacking a covalent link between voltage-sensing and pore domains. Nat Commun 6, 6672.

Lorinczi, E., Helliwell, M., Finch, A., Stansfeld, P.J., Davies, N.W., Mahaut-Smith, M., Muskett, F.W., and Mitcheson, J.S. (2016). Calmodulin Regulates Human Ether a Go-Go 1 (hEAG1) Potassium Channels through Interactions of the Eag Domain with the Cyclic Nucleotide Binding Homology Domain. J Biol Chem 291, 17907-17918.

Ludwig, J., Terlau, H., Wunder, F., Bruggemann, A., Pardo, L.A., Marquardt, A., Stühmer, W., and Pongs, O. (1994). Functional expression of a rat homologue of the voltage gated either a go-go potassium channel reveals differences in selectivity and activation kinetics between the Drosophila channel and its mammalian counterpart. 13, 44514458.

Marques-Carvalho, M.J., Oppermann, J., Muñoz, E., Fernandes, A.S., Gabant, G., Cadene, M., Heinemann, S.H., Schönherr, R., and Morais-Cabral, J.H. (2016). Molecular Insights into the Mechanism of Calmodulin Inhibition of the EAG1 Potassium Channel. Structure $24,1742-1754$.

Martin, S., Lino de Oliveira, C., Mello de Queiroz, F., Pardo, L.A., Stuhmer, W., and Del Bel, E. (2008). Eag1 potassium channel immunohistochemistry in the CNS of adult rat and selected regions of human brain. Neuroscience 155, 833-844.

Mortensen, L.S., Schmidt, H., Farsi, Z., Barrantes-Freer, A., Rubio, M.E., Ufartes, R., Eilers, J., Sakaba, T., Stuhmer, W., and Pardo, L.A. (2015). KV 10.1 opposes activity-dependent increase in $\mathrm{Ca}(2)(+)$ influx into the presynaptic terminal of the parallel fibre-Purkinje cell synapse. J Physiol 593, 181-196.

Movsisyan, N., and Pardo, L.A. (2020). Kv10.1 regulates microtubule dynamics during mitosis. Cancers 12, 1-26. 
Napp, J., Monje, F., Stuhmer, W., and Pardo, L.A. (2005). Glycosylation of Eag1 (Kv10.1) potassium channels: intracellular trafficking and functional consequences. J Biol Chem 280, 29506-29512.

Ng, C.A., Hunter, M.J., Perry, M.D., Mobli, M., Ke, Y., Kuchel, P.W., King, G.F., Stock, D., and Vandenberg, J.I. (2011). The N-terminal tail of hERG contains an amphipathic alphahelix that regulates channel deactivation. PLoS One 6, e16191.

Ninkovic, M., Mitkovski, M., Kohl, T., Stuhmer, W., and Pardo, L.A. (2012). Physical and functional interaction of KV10.1 with Rabaptin-5 impacts ion channel trafficking. FEBS Lett 586, 3077-3084.

O'Connell, D., Mruk, K., Rocheleau, J.M., and Kobertz, W.R. (2011). Xenopus laevis oocytes infected with multi-drug-resistant bacteria: implications for electrical recordings. J Gen Physiol 138, 271-277.

Park, H.Y., Kim, S.A., Korlach, J., Rhoades, E., Kwok, L.W., Zipfel, W.R., Waxham, M.N., Webb, W.W., and Pollack, L. (2008). Conformational changes of calmodulin upon Ca2+ binding studied with a microfluidic mixer. Proc Natl Acad Sci U S A 105, 542-547.

Pellequer, J.L., Wager-Smith, K.A., Kay, S.A., and Getzoff, E.D. (1998). Photoactive yellow protein: a structural prototype for the three-dimensional fold of the PAS domain superfamily. Proc Natl Acad Sci U S A 95, 5884-5890.

Peretti, M., Badaoui, M., Girault, A., Van Gulick, L., Mabille, M.-P., Tebbakha, R., Sevestre, H., Morjani, H., and Ouadid-Ahidouch, H. (2019). Original association of ion transporters mediates the ECM-induced breast cancer cell survival: Kv10.1-Orai1SPCA2 partnership. Scientific Reports 9, 1175.

Peterson, B.Z., Demaria, C.D., Adelman, J.P., and Yue, D.T. (1999). Calmodulin is the Ca2+ sensor for Ca2+-dependent inactivation of L-type calcium channels. Neuron 22, 844844. 
Piros, E.T., Shen, L., and Huang, X.Y. (1999). Purification of an EH domain-binding protein from rat brain that modulates the gating of the rat ether-a-go-go channel. J Biol Chem 274, 33677-33683.

Porro, A., Saponaro, A., Gasparri, F., Bauer, D., Gross, C., Pisoni, M., Abbandonato, G., Hamacher, K., Santoro, B., Thiel, G., et al. (2019). The HCN domain couples voltage gating and cAMP response in hyperpolarization-activated cyclic nucleotide-gated channels. Elife 8 .

Ramos Gomes, F., Romaniello, V., Sánchez, A., Weber, C., Narayanan, P., Psol, M., and Pardo, L.A. (2015). Alternatively Spliced Isoforms of KV10.1 Potassium Channels Modulate Channel Properties and Can Activate Cyclin-dependent Kinase in Xenopus Oocytes. Journal of Biological Chemistry 290, 30351-30365.

Rhoads, R., and Friedberg, F. (2016). Sequence motifs for Calmodulin recognition. The FASEB Journal 11, 331-340.

Rhyner, J.A., Koller, M., Durussel-Gerber, I., Cox, J.A., and Strehler, E.E. (1992). Characterization of the human calmodulin-like protein expressed in Escherichia coli. Biochemistry 31, 12826-12832.

Robertson, G.A., and Morais-Cabral, J.H. (2020). hERG Function in Light of Structure. Biophys J 118, 790-797.

Robertson, G.A., Warmke, J.M., and Ganetzky, B. (1996). Potassium currents expressed from Drosophila and mouse eag cDNAs in Xenopus oocytes. Neuropharmacology 35, 841-850.

Saganich, M.J., Machado, E., and Rudy, B. (2001). Differential expression of genes encoding subthreshold-operating voltage-gated K+ channels in brain. J Neurosci 21, 4609-4624.

Saimi, Y., and Kung, C. (2002). Calmodulin as an Ion Channel Subunit. Annual Review of Physiology 64, 289-311. 
Sánchez, A., Urrego, D., and Pardo, L.A. (2016). Cyclic expression of the voltage-gated potassium channel K V 10.1 promotes disassembly of the primary cilium. EMBO reports $17,708-723$.

Sansom, M.S. (2000). Potassium channels: watching a voltage-sensor tilt and twist. Curr Biol 10, R206-209.

Schönherr, R., Hehl, S., Terlau, H., Baumann, A., and Heinemann, S.H. (1999). Individual subunits contribute independently to slow gating of bovine EAG potassium channels. Journal of Biological Chemistry 274, 5362-5369.

Schönherr, R., Löber, K., and Heinemann, S.H. (2000). Inhibition of human ether à gogo potassium channels by $\mathrm{Ca}(2+) /$ calmodulin. The EMBO journal 19, 3263-3271.

Schönherr, R., Mannuzzu, L.M., Isacoff, E.Y., and Heinemann, S.H. (2002).

Conformational switch between slow and fast gating modes: Allosteric regulation of voltage sensor mobility in the EAG K+ channel. Neuron 35, 935-949.

Sehgal, P., Szalai, P., Olesen, C., Praetorius, H.A., Nissen, P., Christensen, S.B., Engedal, N., and Møller, J.V. (2017). Inhibition of the sarco/endoplasmic reticulum (ER)

$\mathrm{Ca}(2+)$-ATPase by thapsigargin analogs induces cell death via ER $\mathrm{Ca}(2+)$ depletion and the unfolded protein response. J Biol Chem 292, 19656-19673.

Silverman, W.R., Bannister, J.P.A., and Papazian, D.M. (2004). Binding site in eag voltage sensor accommodates a variety of ions and is accessible in closed channel. Biophysical Journal 87, 3110-3121.

Silverman, W.R., Tang, C.Y., Mock, a.F., Huh, K.B., and Papazian, D.M. (2000). Mg(2+) modulates voltage-dependent activation in ether-à-go-go potassium channels by binding between transmembrane segments S2 and S3. The Journal of general physiology 116, 663-678. 
Stefan, M.I., Edelstein, S.J., and Le Novère, N. (2008). An allosteric model of calmodulin explains differential activation of PP2B and CaMKII. Proceedings of the National Academy of Sciences of the United States of America 105, 10768-10773.

Stengel, R., Rivera-Milla, E., Sahoo, N., Ebert, C., Bollig, F., Heinemann, S.H., Schonherr, R., and Englert, C. (2012). Kcnh1 voltage-gated potassium channels are essential for early zebrafish development. J Biol Chem 287, 35565-35575.

Stühmer, W., Conti, F., Suzuki, H., Wang, X., Noda, M., Yahagi, N., Kubo, H., and Numa, S. (1989). Structural parts involved in activation and inactivation of the sodium channel. Nature 339, 597-603.

Stühmer, W., and Parekh, A.B. (1995). Electrophysiological Recordings from Xenopus Oocytes. In Single-Channel Recording, B. Sakmann, and E. Neher, eds. (Boston, MA: Springer US), pp. 341-356.

Sun, X.X., Hodge, J.J., Zhou, Y., Nguyen, M., and Griffith, L.C. (2004). The eag potassium channel binds and locally activates calcium/calmodulin-dependent protein kinase II. J Biol Chem 279, 10206-10214.

Swartz, K.J. (2008). Sensing voltage across lipid membranes. Nature 456, 891-897. Tan, P.S., Perry, M.D., Ng, C.A., Vandenberg, J.I., and Hill, A.P. (2012). Voltage-sensing domain mode shift is coupled to the activation gate by the $\mathrm{N}$-terminal tail of hERG channels. J Gen Physiol 140, 293-306.

Taylor, B.L., and Zhulin, I.B. (1999). PAS domains: internal sensors of oxygen, redox potential, and light. Microbiol Mol Biol Rev 63, 479-506.

Terlau, H., Heinemann, S.H., Stuhmer, W., Pongs, O., and Ludwig, J. (1997). Amino terminal-dependent gating of the potassium channel rat eag is compensated by a mutation in the S4 segment. J Physiol 502 ( Pt 3), 537-543. 
Terlau, H., Ludwig, J., Steffan, R., Pongs, O., Stühmer, W., and Heinemann, S.H. (1996). Extracellular Mg2+ regulates activation of rat eag potassium channel. Pflügers Archiv 432, 301-312.

Thouta, S., Sokolov, S., Abe, Y., Clark, S.J., Cheng, Y.M., and Claydon, T.W. (2014). Proline scan of the HERG channel S6 helix reveals the location of the intracellular pore gate. Biophys J 106, 1057-1069.

Thurman, C.L., Burns, J.S., and O'Neil, R.G. (2000). Identifying the $\mathrm{Ca}(++)$ signalling sources activating chloride currents in Xenopus oocytes using ionomycin and thapsigargin. Cell Signal 12, 629-635.

Tomczak, A.P. (2016). Voltage-gating and assembly of split Kv10.1 channels. In GGNB Göttingen Graduate School for Neurosciences, Biophysics and Molecular Biosciences (Göttingen: University of Göttingen).

Tomczak, A.P., Fernandez-Trillo, J., Bharill, S., Papp, F., Panyi, G., Stuhmer, W., Isacoff, E.Y., and Pardo, L.A. (2017). A new mechanism of voltage-dependent gating exposed by KV10.1 channels interrupted between voltage sensor and pore. J Gen Physiol 149, 577 593.

Trudeau, M.C., and Zagotta, W.N. (2003). Calcium/calmodulin modulation of olfactory and rod cyclic nucleotide-gated ion channels. J Biol Chem 278, 18705-18708.

Ufartes, R., Schneider, T., Mortensen, L.S., de Juan Romero, C., Hentrich, K., Knoetgen, H., Beilinson, V., Moebius, W., Tarabykin, V., Alves, F., et al. (2013). Behavioural and functional characterization of Kv10.1 (Eag1) knockout mice. Hum Mol Genet 22, 22472262.

Urrego, D. (2017). The electric fence to cell-cycle progression: Do local changes in membrane potential facilitate disassembly of the primary cilium? Timely .pdf. 
Urrego, D., Movsisyan, N., Ufartes, R., and Pardo, L.A. (2016). Periodic expression of Kv10.1 driven by pRb/E2F1 contributes to G2/M progression of cancer and nontransformed cells. Cell Cycle 15, 799-811.

Villa, C., and Combi, R. (2016). Potassium Channels and Human Epileptic Phenotypes: An Updated Overview. Front Cell Neurosci 10, 81.

Wang, J., Myers, C.D., and Robertson, G.A. (2000). Dynamic control of deactivation gating by a soluble amino-terminal domain in HERG K(+) channels. J Gen Physiol 115, 749-758.

Wang, J., Trudeau, M.C., Zappia, A.M., and Robertson, G.A. (1998). Regulation of deactivation by an amino terminal domain in human ether-a-go-go-related gene potassium channels. J Gen Physiol 112, 637-647.

Wang, W., and MacKinnon, R. (2017). Cryo-EM Structure of the Open Human Ether-ago-go-Related K(+) Channel hERG. Cell 169, 422-430 e410.

Wang, Z.J., Soohoo, S.M., Tiwari, P.B., Piszczek, G., and Brelidze, T.I. (2020). Chlorpromazine binding to the PAS domains uncovers the effect of ligand modulation on EAG channel activity. J Biol Chem 295, 4114-4123.

Warmke, J., Drysdale, R., and Ganetzky, B. (1991). A distinct potassium channel polypeptide encoded by the Drosophila eag locus. Science 252, 1560-1562.

Whicher, J.R., and MacKinnon, R. (2016). Structure of the voltage-gated K(+) channel Eag1 reveals an alternative voltage sensing mechanism. Science 353, 664-669.

Whicher, J.R., and MacKinnon, R. (2019). Regulation of Eag1 gating by its intracellular domains. Elife 8. 
Wiedemann, C., Kumar, A., Lang, A., and Ohlenschlager, O. (2020). Cysteines and Disulfide Bonds as Structure-Forming Units: Insights From Different Domains of Life and the Potential for Characterization by NMR. Front Chem 8, 280.

Yoshida, S., and Plant, S. (1992). Mechanism of release of Ca2+ from intracellular stores in response to ionomycin in oocytes of the frog Xenopus laevis. J Physiol 458, 307-318.

Zhang, M., Liu, J., and Tseng, G.N. (2004). Gating charges in the activation and inactivation processes of the HERG channel. J Gen Physiol 124, 703-718.

Zhao, Y., Goldschen-Ohm, M.P., Morais-Cabral, J.H., Chanda, B., and Robertson, G.A. (2017). The intrinsically liganded cyclic nucleotide-binding homology domain promotes KCNH channel activation. J Gen Physiol 149, 249-260.

Zhou, L., and Siegelbaum, S.A. (2007). Gating of HCN channels by cyclic nucleotides: residue contacts that underlie ligand binding, selectivity, and efficacy. Structure 15, 655-670.

Zhou, Y., Morais-Cabral, J.H., Kaufman, A., and MacKinnon, R. (2001). Chemistry of ion coordination and hydration revealed by a $\mathrm{K}+$ channel-Fab complex at $2.0 \mathrm{~A}$ resolution. Nature 414, 43-48.

Ziechner, U., Schönherr, R., Born, A.K., Gavrilova-Ruch, O., Glaser, R.W., Malesevic, M., Küllertz, G., and Heinemann, S.H. (2006). Inhibition of human ether à go-go potassium channels by $\mathrm{Ca} 2+/$ calmodulin binding to the cytosolic $\mathrm{N}$ - and $\mathrm{C}$-termini. FEBS Journal $273,1074-1086$. 


\section{Acknowledgements}

It is very difficult to find words to express my gratitude for my mentor Prof. Dr. Luis Pardo. He has once told me that it's not always about the goal and that I shouldn't miss the value of the journey. As I approach the end of my PhD journey, I realize that he has helped me reach my goal and enjoy the journey. I am indeed very grateful.

I would like to thank my thesis committee members Prof. Dr. Heinrich, Dr. Andreas Neef and Prof. Dr. Martin Göpfert for their time and support during the meetings and whenever I asked for help. I am indebted for Dr. Andreas Neef . His interest and enthusiasm have turned the collaboration into an enjoyable and valuable experience. His scientific input was valuable for the project, and I am very grateful for the time and energy he spent teaching me.

I would like to thank my colleagues who helped with this project; Kerstin Dümke for helping with the frog operations, construct synthesis and western blots; Ileana Reséndiz and Araceli Sánchez for guiding me with the biochemistry experiments; Jorge Fernández and Adam Tomczak for teaching me and guiding me at the beginning of the project; my lab rotation students who helped with the recordings (Inés Hojas Garcia-Plaza, Hebatallah Mohamed Abdelrasol, Jenifer Rachel and Çagatay Aydin). I would like to thank everyone in the lab for helping me improve my German. I would like to thank Heide especially for her support. But I am mostly grateful for the friendly atmosphere and for coffee time!

I would like to thank the Neuroscience office (Prof. Dr. Michael Hörner Sandra Drube and Dr. Jonas Barth) for the effort and support through all the organizational work required to complete this journey. I would like to thank IMPRS Neuroscience for granting me a stipend during my PhD studies.

I would like to thank my friends who have supported me during the writing process; Joe, Alaa, Sara, Felix and Naira. I am thankful to Joe for his time and his help. I am also grateful for the movie nights and Netflix parties. I would like to thank my 
friends, who helped me enjoy those last years; I would like to thank: Carolina Léon, for teaching me how to swim. Hong, for our relaxing conversations. I miss our teatime. Xiaoyi , for cheering me up ! Jiayao! Last but not least, I would like to thank my family; my mother" Abeer Mahmoud Salah", my sister Rana and my grandfather "Mahmoud Salah Eldin". Thank you for motivating me and making me smile. I couldn't have reached this far without you. 